\title{
IntechOpen
}

\section{Special Applications of Photogrammetry}

Edited by Daniel Carneiro da Silva 



\section{SPECIAL APPLICATIONS OF PHOTOGRAMMETRY}

Edited by Daniel Carneiro da Silva 
Special Applications of Photogrammetry

http://dx.doi.org/10.5772/1946

Edited by Daniel Carneiro da Silva

\section{Contributors}

Pierre Drap, Daniel Carneiro Da Silva, Ana Lúcia Bezerra Candeias, Benjamin Arias-Perez, Diego Gonzalez-Aguilera, Javier Gomez-Lahoz, David Hernandez-Lopez, Junggeun Han, Kikwon Hong, Sanghun Kim, Rami Al-Ruzouq, Jaechoon Chon

\section{(c) The Editor(s) and the Author(s) 2012}

The moral rights of the and the author(s) have been asserted.

All rights to the book as a whole are reserved by INTECH. The book as a whole (compilation) cannot be reproduced, distributed or used for commercial or non-commercial purposes without INTECH's written permission. Enquiries concerning the use of the book should be directed to INTECH rights and permissions department (permissions@intechopen.com).

Violations are liable to prosecution under the governing Copyright Law.

\section{(cc) BY}

Individual chapters of this publication are distributed under the terms of the Creative Commons Attribution 3.0 Unported License which permits commercial use, distribution and reproduction of the individual chapters, provided the original author(s) and source publication are appropriately acknowledged. If so indicated, certain images may not be included under the Creative Commons license. In such cases users will need to obtain permission from the license holder to reproduce the material. More details and guidelines concerning content reuse and adaptation can be foundat http://www.intechopen.com/copyright-policy.html.

\section{Notice}

Statements and opinions expressed in the chapters are these of the individual contributors and not necessarily those of the editors or publisher. No responsibility is accepted for the accuracy of information contained in the published chapters. The publisher assumes no responsibility for any damage or injury to persons or property arising out of the use of any materials, instructions, methods or ideas contained in the book.

First published in Croatia, 2012 by INTECH d.o.o.

eBook (PDF) Published by IN TECH d.o.o.

Place and year of publication of eBook (PDF): Rijeka, 2019.

IntechOpen is the global imprint of IN TECH d.o.o.

Printed in Croatia

Legal deposit, Croatia: National and University Library in Zagreb

Additional hard and PDF copies can be obtained from orders@intechopen.com

Special Applications of Photogrammetry

Edited by Daniel Carneiro da Silva

p. cm.

ISBN 978-953-51-0548-0

eBook (PDF) ISBN 978-953-51-5603-1 


\section{We are IntechOpen, \\ the world's leading publisher of Open Access books}

Built by scientists, for scientists

\section{$4,100+$}

Open access books available

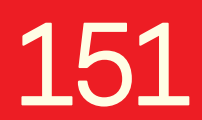

Countries delivered to
$116,000+$

International authors and editors
$120 \mathrm{M}+$

Downloads

Our authors are among the

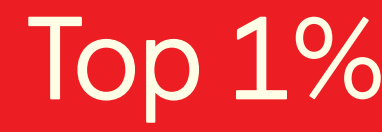

most cited scientists

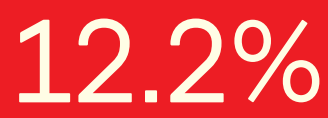

Contributors from top 500 universities

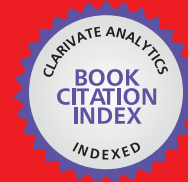

WEB OF SCIENCE ${ }^{\mathrm{TM}}$

Selection of our books indexed in the Book Citation Index in Web of Science ${ }^{\mathrm{TM}}$ Core Collection (BKCI)

Interested in publishing with us?

Contact book.department@intechopen.com

Numbers displayed above are based on latest data collected.

For more information visit www.intechopen.com

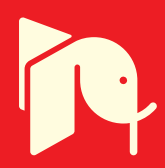





\section{Meet the editor}

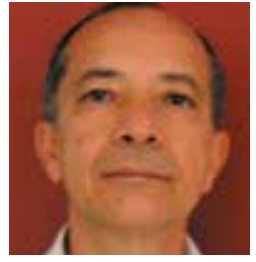

Dr da Silva graduated in Civil Engineering from the University of Pernambuco in 1979, and received his MSc (1993) and DSc (2001) in Geodetic Sciences from Federal University of Parana, Brazil, with a thesis in the field of photogrammetry. He is currently professor of photogrammetry in undergraduate and postgraduate at the Department of Cartographic Engineering, Federal

University of Pernambuco, Brazil. Besides teaching and research activities, Dr da Silva also acts as a consultant in photogrammetric mapping. 



\section{Contents}

\section{Preface XI}

Chapter 1 Color Restoration of Aerial Photographs 1

Daniel Carneiro da Silva and Ana Lúcia Bezerra Candeias

Chapter 2 High-Quality Seamless Panoramic Images 29

Jaechoon Chon, Jimmy Wang, Tom Slankard and John Ristevski

Chapter 3 Assessment of Stereoscopic Precision - Film to Digital Photogrammetric Cameras 47 Benjamín Arias-Pérez, Diego González-Aguilera, Javier Gómez-Lahoz and David Hernández-López

Chapter 4 Application of a Photogrammetric System for Monitoring Civil Engineering Structures 73

Junggeun Han, Kikwon Hong and Sanghun Kim

Chapter 5 Photogrammetry for Archaeological Documentation and Cultural Heritage Conservation 97 Rami AL-Ruzouq

Chapter 6 Underwater Photogrammetry for Archaeology 111

Pierre Drap 



\section{Preface}

Photogrammetry from aerial platforms has been recognized since the 20th century as an important technique to map aerial grades, cities and regions. The modalities known as terrestrial and short distance have also been used, although in lesser proportion, in architecture, to survey historical buildings and monuments.

The main characteristics of photogrammetry, which prevented its wider use in other fields or made it difficult, were the high cost of the equipment - including airplanes, special aerial or terrestrial cameras, support field equipment for topography and geodesy, restitution equipment, and the high labor costs of the highly qualified personnel, from university-educated supervisors to technicians. All steps of the process, from the planning phase and acquisition of photographs to the finalization of the product are composed of a succession of rigorous proceedings, which demand high precision and attention.

This scenario of high operational costs in photogrammetry started to change in the 1990's with the introduction of digital photogrammetry. Already, in the beginning of the second decade of the 21st Century, photogrammetric digital cameras of all sizes are available on the market with high performance computer programs in mapping, but also for specific applications in mechanics, medicine, physiotherapy and other industries. Digital photometry has brought about great gains in productivity and has facilitated its use.

Moreover, with the low cost digital equipment and the freely available advanced processing image programs in libraries, as well as the integration of new developments in the field of information science - computer vision, inertial sensors and GNSS (Global Navigation Satellite System) positioning, it is now possible to develop low-cost personalized solutions. Therefore, photogrammetry is now accessible to non-photogrammetry specialists, researchers, engineers and specialists in all areas who need tridimensional measurements, including those peculiar critical situations in which the object cannot be touched.

The objective of this book is to supply current information about questions and applications of digital photogrammetry. The initial chapters deal with subjects related to radiometric and geometric quality. Chapter one deals with the alterations landscape 
colors suffer in aerial photographs and the methods for their correction. Chapter two deals with methods for eliminating the seam line in mosaics of terrestrial photographs with fish-eye lenses. Chapter three compares and analyzes the precision of planimetric and altimetric measurements in digitalized analogical images and authentic digital images.

The following chapters present examples of applications of terrestrial photogrammetry systems. Chapter four presents a system that can be used to measure the deformations of structural elements in civil engineering, mechanics, and to survey historical buildings. Chapter five shows how the combination of terrestrial, aerial and satellite photographs can be used together to document archaeological sites and historical buildings. Chapter six shows an example of sub-aquatic photogrammetry for archeological surveying and the possibility of recomposing forms of broken and incomplete pieces from a 3D reconstruction. These examples show the solutions adopted for various particular problems each application demands, so as to maximize the data supplied by photogrammetric processing, whether orthorectified images, vectorial designs or a coordinate list.

The editor and the InTech editorial team thank the authors for contributing their work, for the quality of the chapters, the care in proceeding with the revisions, and also to the companies and agencies of governments that made available the photographs, data and materials used in the various papers.

Dr Daniel Carneiro da Silva

Department of Cartographic Engineering, Federal University of Pernambuco, 


\title{
Color Restoration of Aerial Photographs
}

\author{
Daniel Carneiro da Silva and Ana Lúcia Bezerra Candeias \\ Federal University of Pernambuco \\ Brazil
}

\section{Introduction}

A non-uniform distribution of illumination on a negative is provoked by direct and indirect illumination, atmospheric factors and construction of lenses. The effect in aerial photographs can be perceived more easily in photo-indices and mosaics. Often it is attributed to vignetting. However, the direction of illumination due to the position of the sun and atmospheric factors provoke an additional effect that is not radially symmetric to the center of the photograph. This compound effect can appear in any photograph scale.

These problems that were well resolved with the use of filters for haze or anti-vignetting in black\&white photographs, are now becoming more critical with the current wide use of color photographs and the increasing use of orthophoto-maps produced with those photographs.

There was also the adaptation of the production methodology of the orthophoto-maps, which in the past demanded that each sheet be produced with just one photograph, but which nowadays, by using the techniques of digital image processing, can allow mosaics of two or more photographs. There is a demand nowadays for seams not to be apparent because of the common differences in tones that exist between neighboring photographs.

The techniques that are already available can resolve various problems, such as the reduction of clearness because of haze from sun reflection, bright areas (hot spots) and vignetting effect; with digital image processing for commercial programs (Nobrega \& and Quintanilha, 2004; Li et al., 2004a; Wu \& Campbell, 2004; Paparodis et al., 2006), but the results are not always acceptable, because the seams are visible or because artifacts appear. On the other hand, there are a lot of research studies and methods being developed that have presented good results and can be incorporated into commercial programs. Some of these methods are discussed in LI et al. (2004a).

This chapter is divided into three main parts: the first one shows the causes of non-uniform illumination in aerial photographs; the second one shows the practical applications of some methods; and the third one presents the results.

Initially, the correction of the vignetting effect is presented by a simple formula and the haze effect in high altitude photographs with color transformation. After that, a method is developed based on masks that is intended to correct the combined illumination degradation effect of vignetting with the bi-directional reflectance distribution function 
(BRDF) in color aerial photographs. The manipulation of histograms and the Kries hypothesis are showed for color transformation applied to aerial photographs.

The results are discussed in terms of visual quality and processing time.

\section{Non uniform illumination in aerial photographs}

The non uniform illumination in aerial photographs originates in the vignetting effects, directional scattering of solar illumination in the presence of haze and the surface bidirectional reflectance.

Moreover there are other factors that can reduce the quality of aerial photographs during their execution, such as clouds or shadows from clouds, shadows from topographic elevations or buildings, reflection from the sun in water bodies, smoke, haze and the quality of the optical system and the film. Analogical aerial photogrammetry developed ways to avoid or partially correct those problems with the use of devices like filters and special films, aside from adequate flight planning for each region and season of the year.

Nowadays, some of these problems, like the reduction of clearness by haze, reflexions from sunlight, shiny areas (hot spot) and vignetting, can be solved, at least partially, with digital image processing programs (Nobrega \& Quintanilha, 2004; Lamparelli et al, 2004; Silva \& Candeias, 2008; Li et al., 2004a). Other aspects are more complex, like the elimination of cloud shadows, and they are still being studied (LI et al, 2004b).

\subsection{Vignetting effects}

The vignetting effects come from the non-uniform illumination that passes through a lens system until it reaches the negative, where the amount of light is greater in the center and diminishes at the borders. The effect is radial and symmetrical in the center of the photograph, the borders become darker, and in the case of colored photographs, they also become bluer. This problem is greater for wide angle cameras. Figure 1 presents an example of the vignetting effect in black and white and color photograph.

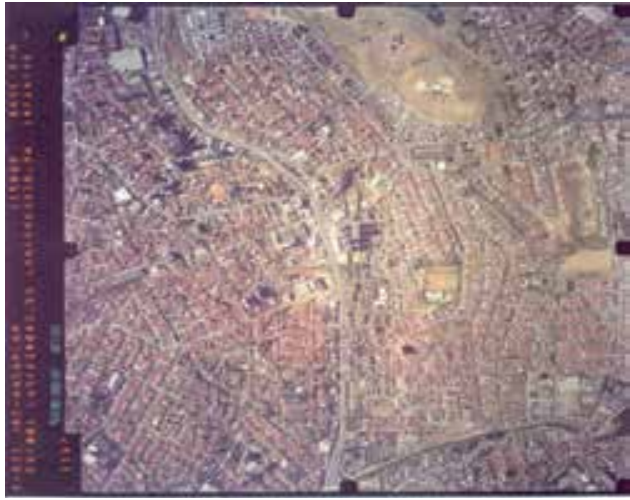

a) Colored

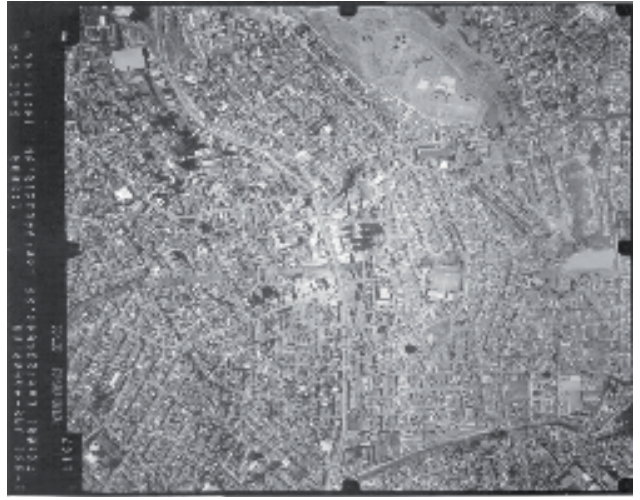

b) Black and white

Fig. 1. Photograph with vignetting effect obtained with wide angle camera. (Photograph: Base Engenharia) 
As the effect is symmetrical in relation to the center of the photograph, it can be mathematically corrected. Equation 1 is a very used function to correct this problem

$$
I(b)=\cos (b)^{n}
$$

Where: $I$ is illumination which reaches the negative

$b$ angle between optic axis and light ray

$n$ varies from 2.5 to 4 (Slater, 1983; Kraus, 1997).

Equations like the one above were used also by HOMMA et al. (2000); Homma et al. (2000); Lamparelli (2006) for correction of the vignetting effect in aerial photographs.

\subsection{Effects of atmospheric radiance in aerial photographs}

The effects of atmospheric radiance in aerial photographs are complex and they are caused by camera altitude, type, size, concentration and distribution of the atmospheric aerosol, sighting angle, height and azimuth in relation to the sun (Slater, 1983). They can be uniform or non-uniform in the whole area of the photograph. Normally, for photographs from great heights the uniform effect is attributed to the haze and the non-uniform area is related to BRDF (Bi-directional Reflectance Distribution Function) and variations in the type of haze (Paparoditis et al., 2006; Wu \& Campbell, 2004).

Figure 2a shows the geometric elements of an aerial photograph that will be useful for the discussion of this study: the EC (Exposition Center), the Nadir, the solar height angle, sun rays indicated by arrows and the camera viewing angle. In Figure $2 b$, the solar azimuth and the direction of the illumination in relation to the EC are shown.

In the next section we will analyze the effects of atmospheric radiance in aerial photographs of low and high altitude.

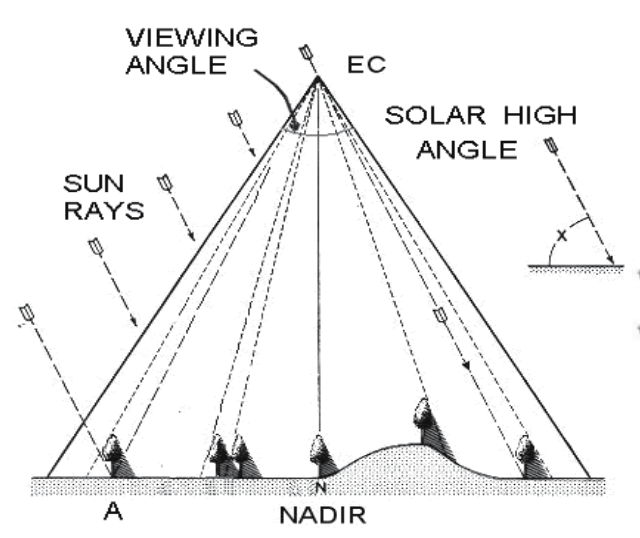

a)

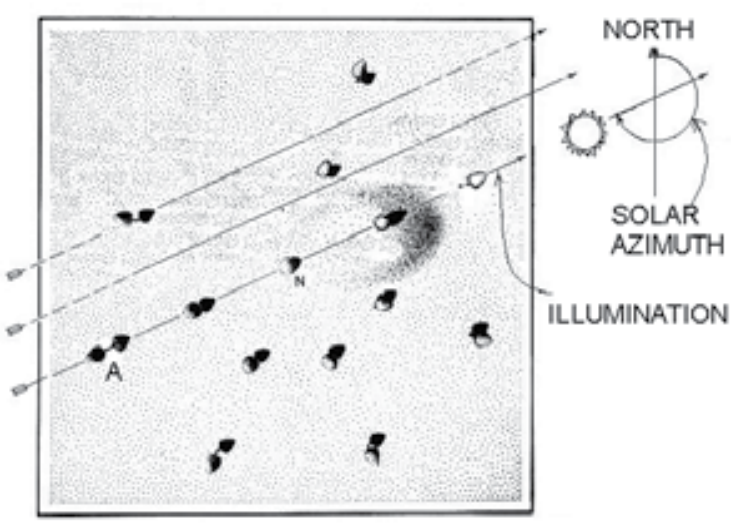

b)

Fig. 2. Geometry of solar illumination on vertical aerial photograph

\subsubsection{Haze effect}

A well known effect of haze in photogrammetry is the reduction of contrast in photographs taken at high altitudes and in the case of colored photographs the appearance of a uniform 
blue tone (Figure 3). This effect is produced by light scattering in the atmosphere even with a clear sky and it is increased in the presence of a dry or humid haze. As a blue light has a higher index of refraction, its scattering is greater and it becomes more visible (Slater, 1983; Fiete, 2004). The reduction of the contrast is significant and reduces the visualization of details of the images (Kraus, 1992).

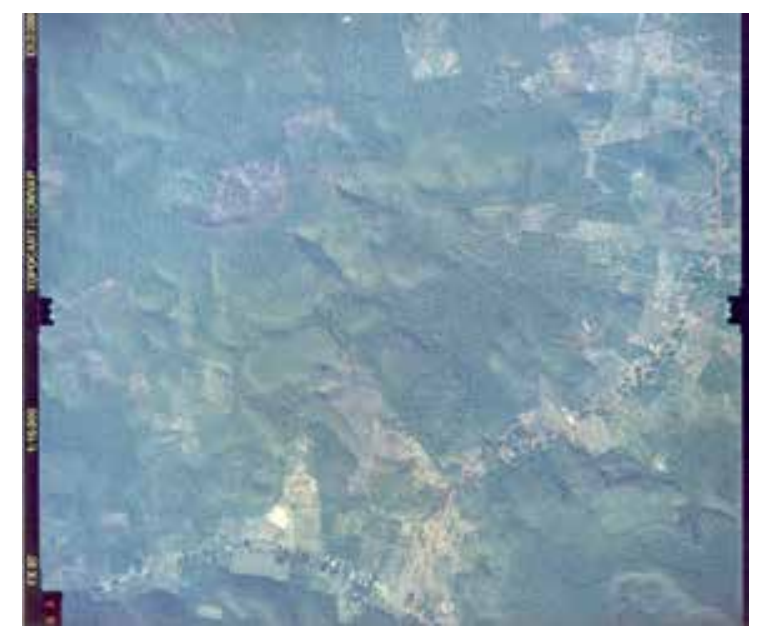

Fig. 3. Bluish color Photograph due to the presence of haze in the atmosphere (Photograph: TOPOCART S/C)

\subsubsection{Bright areas}

Bright areas, more known as hot spots, are the effect caused by the non-visualization of object shadows due to the position of the observer in relation to the sun. This type of bright area does not have to be confound to specular reflection which will be discussed in the next section.

When the sun is directly behind the EC or the observer, a great portion of the landscape will be visualized with direct lighting, and the reflectance will tend to be greater. At the same time the shadows are covered by the height itself of objects like buildings and trees. This effect is due to Paparoditis et al., 2006; Beisl \& Woodhouse, 2004). Figure 4

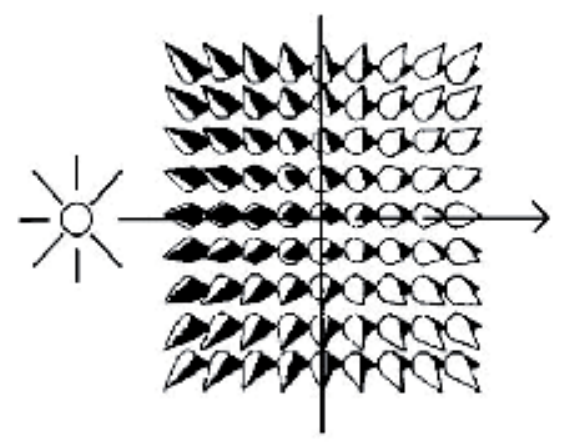

Fig. 4. The brighter area, in the right side of the figure, is in opposite side to the sun due to BRDF. (Tuominen \& Pekkarinen, 2004). 
illustrates the effects of direct illumination in uniformly spaced trees, which causes an illumination gradient. The sun is on the left in relation to the center of the photograph., so the illumination is darker. On the opposite side, on the right, the BRDF effect occurs and the illumination is brighter.

The BRDF evaluates the reflectance of a surface and depends on the direction of the irradiant flux and the direction of the reflected flux detected (Slater, 1980). This evaluation considers the height angles and the sun azimuth, the angles of the surface on which the flux focuses, the orientation angles and the wave length of the visible light. The calculation of the BRDF is complex and is often used in illumination models, the greater difficulty being the need for information about the reflectance and shape of the objects in the terrain, that are not easily available. As an alternative, simplified or empirical functions are used to estimate the effects of the BRDF in aerial photographs (Beisl \& Woodhouse, 2004).

The effects of the BRDF have an impact in the quality of the images in the same magnitude order as the atmospheric effects (Beisl \& Woodhouse, 2004), including the haze.

The shape of the hot spot is normally reported as a bright circular area or a peak (Asrar, 1989; Beisl \& Woodhouse, 2004), but as can be seen in the illustration of Figure 4 and the examples in Figure 5, the lighter areas do not have a regular circular shape. In vertical images it always appears when the solar zenithal angle is smaller than the sight angle camera.

The black circles on figures $5 \mathrm{a}$ and $5 \mathrm{~b}$ are plotters of viewing angles with a $10^{\circ}$ interval and zero at center, showing the subjective visualization of the BRDF effect. The sun position was known from flight data so that the arrow is pointing to the sun and the center of the white circle coincides with the solar zenith angle. These figures also help the visualization of the directional spreading of the haze effect. As will be seen in section 2.2.4 the haze increases the BRDF effect.

\subsubsection{Points of specular reflection}

The bright peaks, as seen in left side of Figure $5 \mathrm{~b}$, can be caused by the specular reflection of the sun in water bodies or metallic rooftops. They are also some times called hot spots in the literature, but they occur in the same side of the sun in the image. That is why in this chapter we will call them of points of specular reflection.

Specular reflection points appear when the solar high angle is bigger than half of the opening angle of the camera and the projection of the sun rays reaches a reflective surface. Using the geometry in Figure 2 this reflection would occur in point A. When using a large angular camera there can be reflection points with the sun height at over $45^{\circ}$, which is why it is necessary to take special care on photogrammetric flights done around midday, when the sun is higher. In the photograph of figure $5 \mathrm{~b}$ there are both specular reflection points (pointed by arrow) and hot spots (neighborhood of the white circle), positioned on the opposite side in relation to the center of the photograph.

\subsubsection{Directional spreading of light of the haze effect}

Some authors noted that there are other factors besides the BRDF that can change the illumination in photographs. Kraus (1989) shows that the photographs taken in the Southern 
and Northern hemispheres present systematically brighter areas in the North and South, respectively. Asarar (1989) shows that there is more radiation spread in the direction close to the incident light that must not be confused with the hot spot effect.

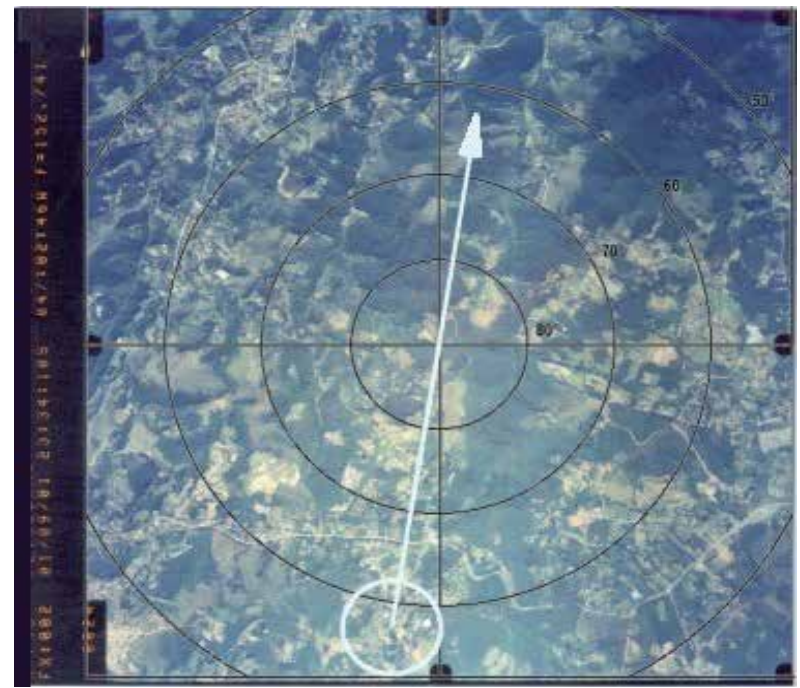

a)

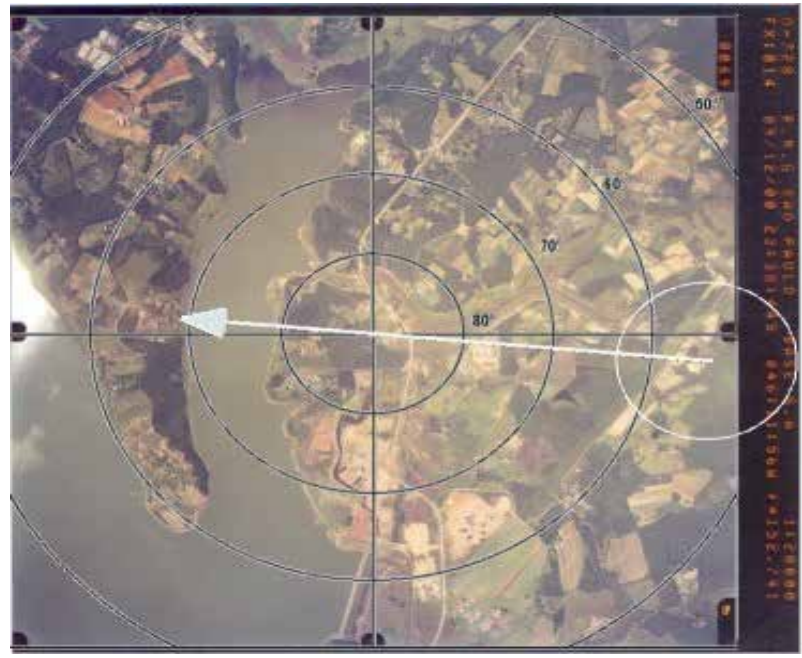

b)

Fig. 5. White circle on center of hot spot and arrow pointing to sun. a) Only hot spot, b) Also with specular reflection on side of sun. (Photograph: Base Engenharia)

Silva \& Candeias (2009) have reproduced the work of Hall (1954), which allows the identification of the center of bright areas which is the effect of haze scattering and a function of the angle of the sun light and viewing angle of camera.

The radiance reaching the sensor is the sum of spectral reflectance and thermal radiant surface, multiplied by the spectral transmittance of the path in the atmosphere (Slater, 1980). 
The product is added to this spectral radiance upward along the route. Thus the formulation presented by Hall contains simplifications and does not take into account the multiscattering. However, their approach is very enlightening and shows that the flow or final radiance is a function of the scattering coefficient and the distance traversed by the flow.

Hall (1954), using G. B. Harrison's formulas, relates the haze factor to the height of the sun. To do that is need consider the brightness of alight pulse sweeping a vertical plane as in the Figure 6 . The geometric elements of figure 6 involved in the formulas are: the sun zenith angle $\theta$, the a angle $\Phi$ that makes a haze cone with the sun ray, the viewing angle $\beta$, the exposition center $(E C)$; altitude of flying $h$. The points on the ground A and B have viewing angles $\beta a$ and $\beta b$, respectively, and point $\mathrm{N}$ is in Nadir.

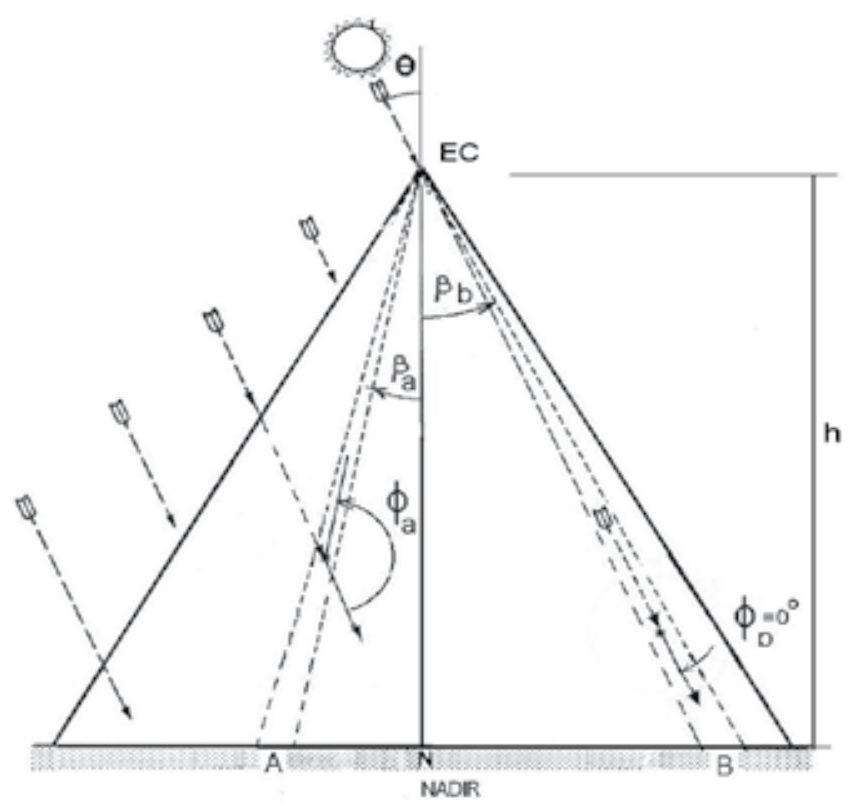

Fig. 6. Geometrical elements involved in analysis of haze factor.

The percentage of haze factor at $\beta$ angle can be estimated in aerial photographs by:

$$
H \beta=100 \times\left(H_{B} \beta\right) / G B \beta
$$

Where: $H^{B} \beta$ is the brightness of an elemental cone of haze which makes an angle $\beta$ with the vertical line or nadir

$G^{B} \beta$ is the brightness as seen through the haze of a horizontal white diffuse reflector on the ground intercepted by the cone.

The brightness of a cone of haze at angle $\beta$ is given by:

$$
H^{B} \beta=A f(\phi) \frac{\cos \theta}{\cos \beta+\cos \theta}\left[1-e^{-\sigma h(\sec \beta+\sec \theta}\right]
$$

Where: $\sigma$ is the coefficient of total scattering in the $\mathrm{ft}^{-1}$ unit. 
$f(\Phi)$, is the scattering phase function, which describes the angular distribution of scattered radiation ( $\Phi$ here is the scattering angle). The scattering phase function $f(\Phi)$ depends on the density and physical characteristics of the particles and the wavelength. As you increase the size of the particles there is a stronger forward scattering $\left(\Phi=0^{\circ}\right)$ and a smaller but still significant back-scattering $\left(\Phi=180^{\circ}\right)$.

Doing the second part of left side of (3) as:

$$
G=\frac{\cos \theta}{\cos \beta+\cos \theta}\left[1-e^{-\sigma h(\sec \beta+\sec \theta}\right]
$$

We now have the brightness of the haze as:

$$
H^{B} \beta=A \cdot f(\phi) \cdot G
$$

The brightness of a white reflector is given by:

$$
G^{B} \beta=A \frac{\cos \theta}{\pi}\left[e^{-\sigma h(\sec \beta+\sec \theta}\right]
$$

The haze factor in percentage is therefore:

$$
H \beta=100 \pi f(\phi) \frac{\left[e^{\sigma h(\sec \beta+\sec \theta)}-1\right]}{\cos \beta+\cos \theta}
$$

Where $A$ is a constant and depends of sun illumination.

The locus of $G$ with respect to $\beta$ is a smooth curve with minimum at $\beta=0$ and symmetrical of about $O Y$.

The curve of $\mathrm{H}^{\mathrm{B}} \beta$ for $\theta=40^{\circ}, \sigma=0.00005 \mathrm{ft}^{-1}, h=16000 \mathrm{ft}$ is shown on Figure 7 . The value of $\sigma$, estimated at $5 \times 10-1 \mathrm{ft}^{-1}$ corresponds to the daytime visibility of 20 miles. The curve of $f(\Phi)$ against $\Phi$ is generally $U$ shaped and the values used by Hall are in Table 1.

\begin{tabular}{|c|c|c|c|c|c|c|c|c|c|c|}
\hline$\Phi$ & 0 & 10 & 20 & 30 & 40 & 50 & 60 & 70 & 80 & 90 \\
\hline$f(\Phi)$ & 0.470 & 0.400 & 0.310 & 0.220 & 0.160 & 0.110 & 0.070 & 0.050 & 0.040 & 0.035 \\
\hline$\Phi$ & 100 & 110 & 120 & 130 & 140 & 150 & 160 & 170 & 180 & \\
\hline$f(\Phi)$ & 0.030 & 0.030 & 0.040 & 0.045 & 0.060 & 0.080 & 0.100 & 0.160 & 0.030 & \\
\hline
\end{tabular}

Table 1. Values of scattering phase function $f(\Phi)$ against $\Phi$ used by Hall(1954).

The strong variation over a short range of $\beta$ in the neighbourhood of $\beta=-\theta$ and $\Phi=180^{\circ}$ means a variation which is proportional to the haze lightness that illuminates the corresponding region of the photograph (Hall, 1954).

Figures 8a and $8 \mathrm{~b}$ show graphs of the haze factor percentage calculated for $\sigma=0.00005 \mathrm{ft}^{-1}, \sigma$ $=0.00008 \mathrm{ft}-1, h=16,000 \mathrm{ft}$, angles $\theta=40^{\circ}$ and $\theta=60^{\circ}$. The haze factor is always greater on the side opposite the sun $\left(\beta=-40^{\circ}\right.$ in figure 8a). There is a sharp peak when $\sigma$ increases around $\theta=40^{\circ}$. If the semi-lens viewing angle $(\beta)$ is $45^{\circ}$ and $\theta=60^{\circ}$ the maximum haze factor would be out of the image. (Figure $8 \mathrm{~b}$ ). 


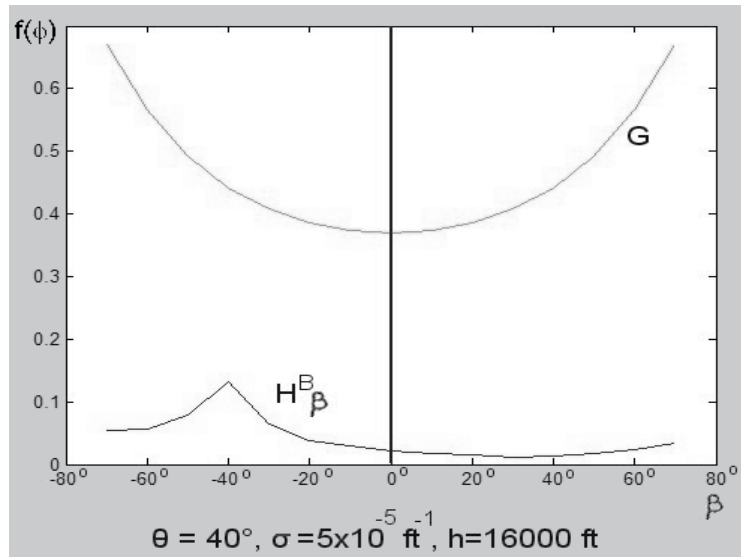

Fig. 7. Brightness curve of the haze $(H B \beta)$ with $A=1, \theta=40^{\circ}, \sigma=0.00005 \mathrm{ft}^{-1}, h=16,500 \mathrm{ft}$

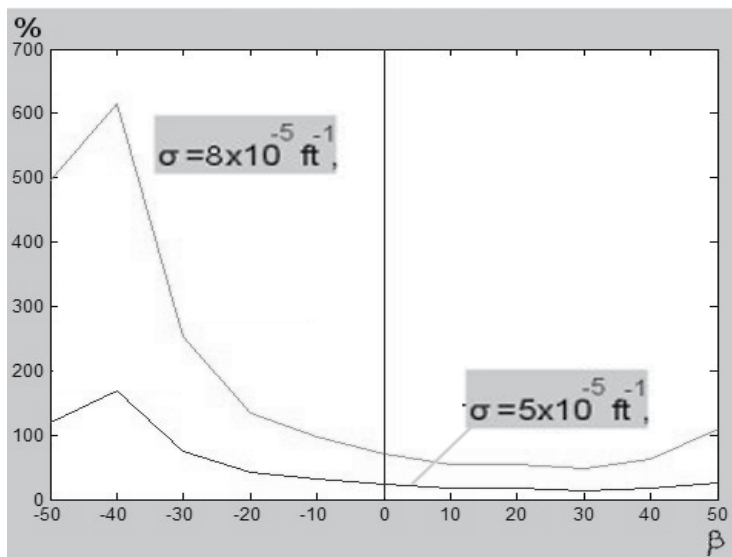

a)

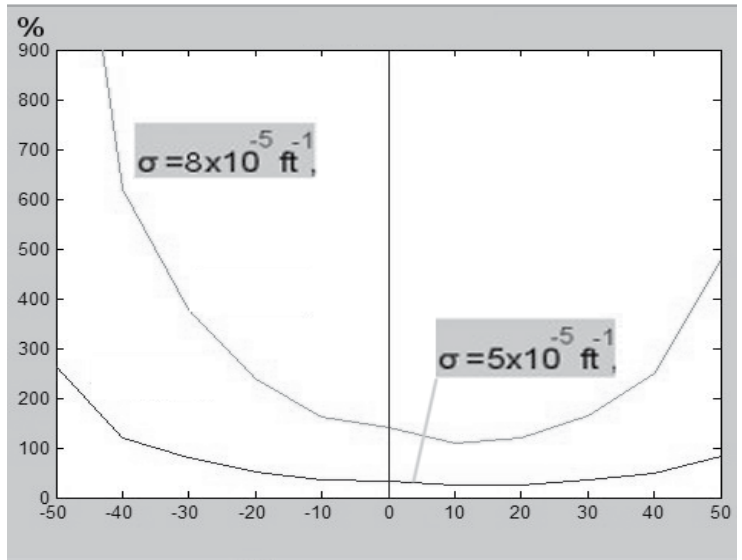

b)

Fig. 8. Curves of the factor haze percentages with $\sigma=0.00005 \mathrm{ft}^{-1}, \sigma=0.00008 \mathrm{ft}^{-1}$, $\mathrm{h}=16,000 \mathrm{ft}$. a) $\theta=40^{\circ}$ b) $\theta=60^{\circ}$ 
With the given formulas it is possible to estimate the positions of the brighter areas and the gradient of change of lighting in the photographs, using data from flying reports and sun ephemeris.

\section{Restoration of colors}

The colors of objects and landscapes in the photographs altered by any of the various factors discussed above can be restored, at least in part, with the help of processing image methods, so that they are more realistic, more uniform between adjacent photos and more uniform with respect to the frame itself.

In this section some of these procedures will be applied. In photogrammetry and remote sensing the methods used in general are: application of functions to correct vignetting (Homma et al, 2000), models of atmospheric radiation and illumination (Beisl\&Woodhouse, 2004), manipulation of histograms (Kraus, 1997; Tuominen \& Pekkarinen, 2004; Nobrega \& Quintanilha, 2004), restoration with the use of RGB color space, HSI-RGB (Guo \& Moore, 1993), use of masks (Li et al, 2004a, Silva \& Candeias, 2008), burning and dodging (Reinhard et al, 2002; Li et al, 2004a).

\subsection{Restoration of haze}

During the taking of the photographs the attenuation of haze effects can be done using a yellow filter placed in front of the lense that absorbs the excess blue light. In digital photogrammetry the degradation, in digitalized images or those obtained directly from a digital camera, can be corrected using radiometric processing functions, color correction or radiometric atmospheric models. Figure 9 shows an original photograph and the result after color casting correction using Kries method explained in section 3.6.

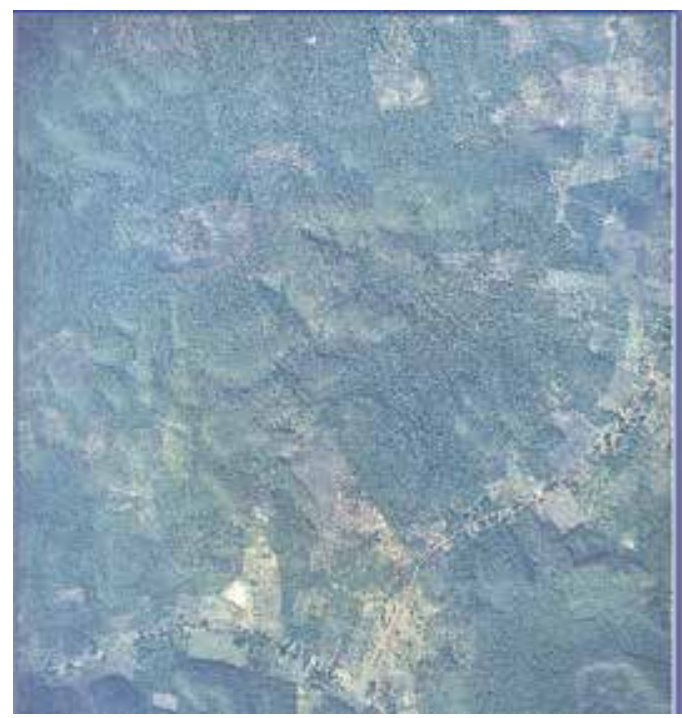

a)

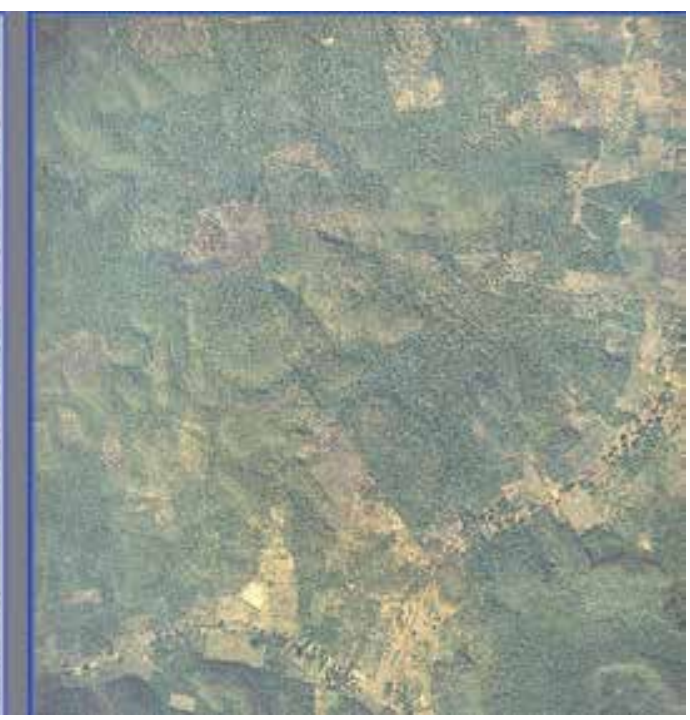

b)

Fig. 9. a) Photograph with uniform haze, b) Photograph processed using Kries method. 


\subsection{Corrections of specular reflection}

Peaks of sun light create white spots or white areas on digital images when the amount of concentrated light exceeds the dynamic range of sensitivity of the CCD sensor. This problem can be avoided with proper planning of the photogrammetric flight, knowing of sun ephemeris and, nowadays, it can be mitigated with the use of digital images with more than eight bits per color channel. Ashikhmin (2002) and Reinhard et al. (2002) can make details visible in parts that are too light or too dark, using algorithms that adapt contrast intervals, to map levels of the original image to the levels likely to be played on the monitor screen or in print.

\subsection{Correction by manipulation of histogram blocks}

Among the processes that can correct non-uniform illumination in digital images, that are available in commercial programs, the most common is the one that carries out the balancing of histograms between blocks, through homogenization of statistical parameters. The correction is done by dividing the original images into blocks, or into sub-images, calculating diverse statistics as global minimum and maximum averages of each block; and processing the histograms, so that they balance the differences of brightness intensity among the blocks. This method can be used both among photographs of a mosaic andin isolated photographs for correcting vignetting effects, bright areas and BRDF. A description of this method, together with some examples of variation, can be found in Li et al., (2004); Paparodis et al. (2006) and Nobrega \& Quintanilha (2004).

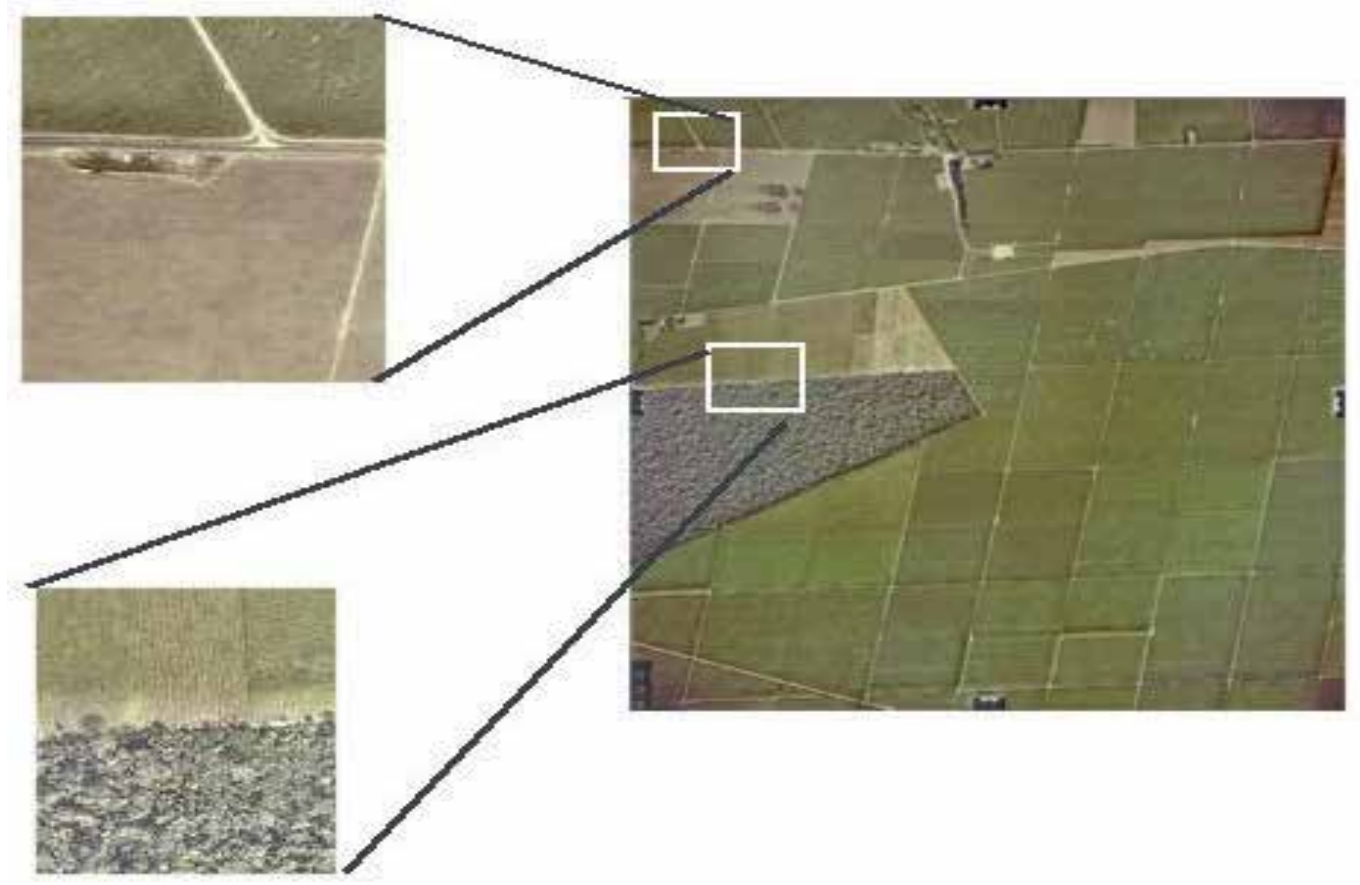

Fig. 10. Photograph processed using histograms blocks balancing, showing transitions between areas with significant tone differences. (Photograph: TOPOCART S/A) 
The method, although well diffused, does not always have good results, principally if the photographs, or areas of photographs, have significant intensity differences or abrupt variations in tonalities. This problem can be seen in examples shown in (Nobrega \& Quintanilha,2004; Li et al., 2004a; Wu \& Campbell, 2004; Paparodis et al., 2006). Figure 10 shows an example of a photograph processed with this type of manipulation of histograms. The amplified details show the color gradients which appear in the transition zones between clear and dark features in the images, and among different kinds of vegetation.

The algorithms to manipulating of histograms blocks divide the image into sub-images, and after that it calculates parameters such as mean, standard deviation, minimum and maximum gray levels of each color channel and as individual histograms. There is a variation called LRM (Local Range Modification) and the definition is presented in Schowendgert (1997). In this process, after the standardization of histograms, a smoothing process near the edges of each sub-image is adopted to avoid the appearance of breaks in continuity. Thus the maximum (MAX) and minimum (MIN) values are calculated for each vertex of the block, the minimum average (LA, LB, LD, LE) and average maximum values $(H A, H B, H D, H E)$ of neighboring blocks, as shown in Figure 11.

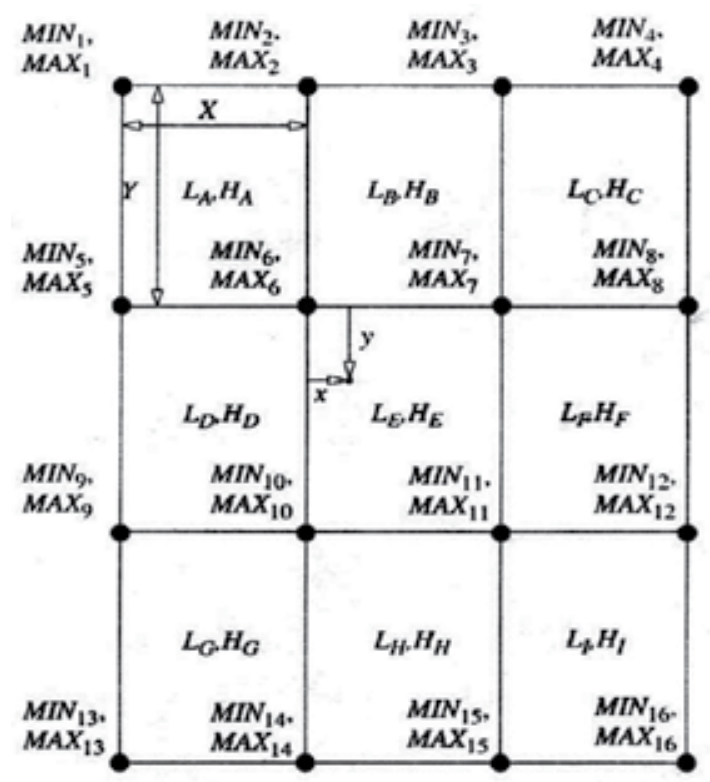

Fig. 11. Blocks and parameters used in the LRM (Schowengerdt, 1997).

The interpolation for the $x y$ position in the above figure uses the maximum and minimum values of the vertices $6,7,10$ and 11 and provides the values given by the equations below:

$$
\begin{aligned}
G L \min & =\left[\frac{x}{X} M I N_{7}+\left(\frac{X-x}{X}\right) M I N_{6}\right]\left(\frac{Y-y}{Y}\right)+\left[\frac{x}{X} M I N_{11}+\left(\frac{X-x}{X}\right) M I N_{10}\right] \frac{y}{Y} \\
G L \max & =\left[\frac{x}{X} M A X_{7}+\left(\frac{X-x}{X}\right) M A X_{6}\right]\left(\frac{Y-y}{Y}\right)+\left[\frac{x}{X} M A X_{11}+\left(\frac{X-x}{X}\right) M A X_{10}\right] \frac{y}{Y}
\end{aligned}
$$


Where: GLmin = minimum value of brightness of the pixel.

GLmax = maximum value of brightness of the pixel

$X=$ dimension of the block columns

$Y=$ dimension of the block in rows

$x=$ column for the position of the pixel in the block

$y=$ row for the position of the pixel in the block

The calculated values are used in the interpolations to get the new gray value image produced:

$$
G L^{\prime}=\frac{255}{G L \max -G L \min }(D N-G L \min )
$$

Where: $G L^{\prime}=$ new value of brightness to be applied to pixel.

$D N=$ value of brightness of the pixel

This algorithm provides good results but has high computational cost and some variations have been proposed that use only mean and standard deviation compared with a global mean value. In general, all promise gain of brightness for darkened areas and darkening of light regions. This type has been used in commercial programs for balancing color aerial photographs and satellite images and digital mosaics in general. For more details consult Nobrega \& Quintanilla (2001).

\subsection{Global histogram manipulation}

The more common methods of manipulations of histogram are equalization and stretching (or normalization). They are applied for enhance contrast of images. Histogram Equalization seeks to produce a balanced image with a uniform distribution of grey tones. Histogram normalization adopts the normal distribution as the transfer function for the desired image.

Furthermore is possible to change the histogram of an image using the histogram of another image as model. This procedure is called histogram matching and figure 12 shows a example. The histogram of figure 12a is of desired image, used as reference, in figure $12 \mathrm{~b}$ is the histogram of image to be changed and in figure 12c is the histogram of new image. The final result is very similar to figure $12 \mathrm{a}$.

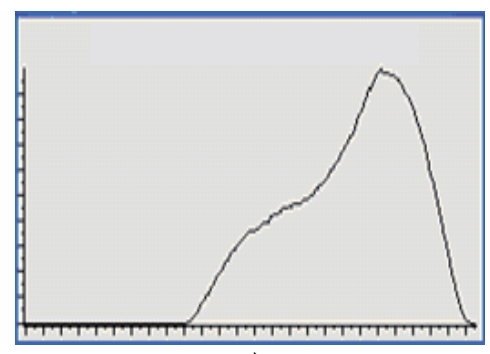

a)

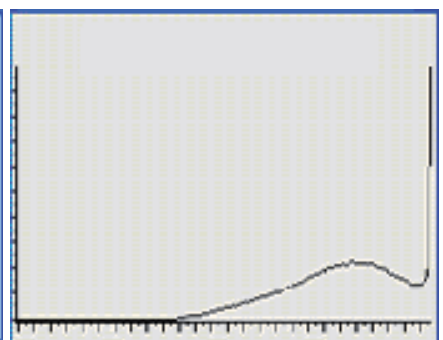

b)

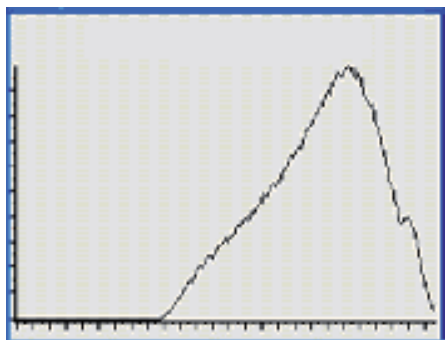

c)

Fig. 12. Example of histogram matching. a) Histogram of reference image, b) Histogram to be changed, c) Histogram changed. 
Most of image processing software has the capacity of handle with histograms and change them. More details about theory can be found in (Kraus, 1997; Pratt, 1991).

\subsection{Correction with masks}

The masks in image processing are binary images or gray tones that are used for delimiting areas where certain operations can be carried out, or to control the degree of processing they can go through. Examples of binary image masks are the delimiting polygons of each of the images that form mosaics. Masks in gray tones are already used by photographers and the graphic industry for attenuating shadows of scenery and environment.

However, as these resources have not been used in photogrammetry, an analysis of the viability of the use of masks is carried out to correct non-uniform illumination in aerial photographs. For this, initially, a complete sequence of a mask construction will be detailed.

A mask should represent the mean illumination intensities of the combined effects of vignetting and DBRF. Considering that the variation in luminosity which reaches the plan of the negative does not vary significantly among the photographs of the same area or when taken in a short period of time, a mask should suffice for processing a group of neighboring aerial photographs.

For the construction of the masks (Figure 13) only one photograph would be used, but the mask can get better if two or more are used, so that the result can be free of differences in the tonality of the scenery. The process can start by two ways: a) profiles of gray values of the image pixels in lines and columns, along the borders, through the center and intermediate regions; b) or using averages values of sub-blocks. This latter process eliminates better the high frequencies. Therefore, when using polynomial regression of a cubic function, as in equation 10, an adequate fitting for a uniform surface is obtained without abrupt changes:

$$
\mathrm{Z}(x, y)=A+B y+C y^{2}+D y^{3}+E x+F x y+G x y^{2}+H x^{2}+I x^{2}+J x^{3}
$$

Where: $x, y=$ coordinates of the points equivalent to the positions of the pixels or centers of sub-blocks

$Z(x, y)=$ the ordinate, or grey value, in the $x, y$ position

$A$ to $J=$ the coefficients obtained with a sample entry.

The masks can be constructed in accordance with the available resources and the ones used here were obtained by following the steps below:

- $\quad$ Transformation of colored images into gray tones at levels of 0 to 255 .

- Division of image forming a grid of 5x5 (25 blocks), trying to eliminate the margins and the fiducial marks.

- Calculation of mean intensity of each of the 25 blocks and registration of the minimum and maximum levels.

- Interpolation of $Z$ values with a cubic or quadratic function.

- conversion to negative image 

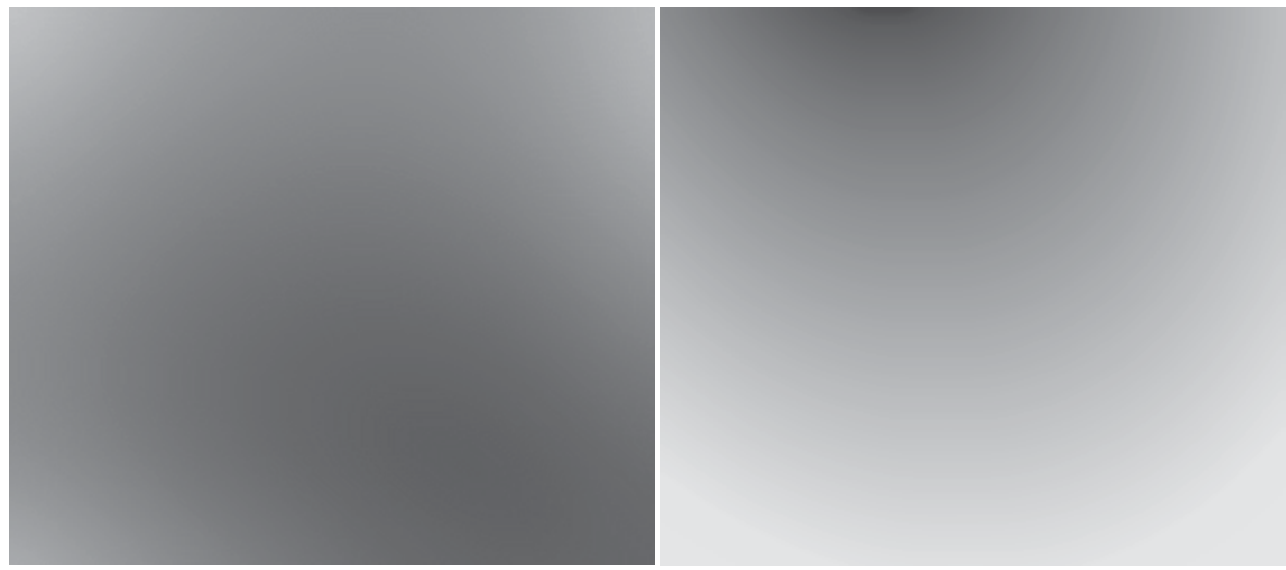

Fig. 13. Examples of masks to correcting illumination intensities.

\subsection{Corrections using Kries method}

The correction or alteration of colors in digital images car be carried out through several methods using operations in color space and transformations between them, however, a good experience of the human operator is necessary. A method that could be more independent is based on the Von Krie hypothesis.

The Von Krie hypothesis method considers that the primary stimuli of $R G B$ color in the retina can be linked to the imaginary stimuli $(X Y Z)$ by a linear transformation with matrix M (Wyszecki \& Stiles, 1982). Equation 11 shows the relation of $R G B$ with $X Y Z$.

$$
\left[\begin{array}{l}
R \\
G \\
B
\end{array}\right]=M\left[\begin{array}{l}
X \\
Y \\
Z
\end{array}\right]
$$

This hypothesis is commonly used in image acquisition devices (such as cameras and scanners) to correct image lighting (sunlight, incandescent or fluorescent lamps) in a simplified manner, in two ways:

A) white balancing

This method is known as image white balancing

$$
\left[\begin{array}{c}
R^{\prime} \\
G^{\prime} \\
B^{\prime}
\end{array}\right]=\left[\begin{array}{ccc}
k R & 0 & 0 \\
0 & k G & 0 \\
0 & 0 & k B
\end{array}\right]\left[\begin{array}{l}
R \\
G \\
B
\end{array}\right]
$$

Where $R^{\prime} G^{\prime} B^{\prime}$ is image desired, and $R G B$ is the original image. The coefficients are obtained from the sample initially and then applied to the entire image

$$
k R=\frac{R^{\prime}}{R} ; k G=\frac{G^{\prime}}{G} ; k B=\frac{B^{\prime}}{B}
$$


Where $R, G, B$ are the average of the desired area. $R^{\prime}=G^{\prime}=B^{\prime}=255$, because they correspond to a white area and to images of eight bits per channel.

B) When the lighting is not known

In this case the process is applied in a representative area of the image and the maximum level of intensity in each band is determined. With this procedure $R=\max (R), G=\max (G)$ and $B=\max (B)$ and $R^{\prime}, G^{\prime}$ and $B$ 'are also equal to 255 .

In this study the method was applied to change the colors of an image with undesirable color based on another image that shows the desired tone, or even the same image in different areas.

\section{Results and discussion}

\subsection{Vignetting correcting}

Vignetting corrretion was presented in Equation 1 . The image in grey format is sized for $1 / 8$ of original size, and applied a filter like Gaussian to blur the image and to get the grey levels in the center and the borders. The final calculations use Equations 14 and 15 with the following adaptations: a) consideration of the maximum radius equal to diagonal of image frame and each pixel radius equal distance between pixel and image centre; $b$ ) for simplicity, $\cos (b)$ calculation uses the focal length equal to the width of the image; $c)$ the maximum correction occurs at border and at center it is null.

During the calculation the radius is obtained and the correction for each pixel is given by fcor. The radius is the distance pixel-center. The gray level correction is:

$$
f \operatorname{cor}=\operatorname{corr} \max ^{*} \frac{(1-\cos \beta)}{(1-\operatorname{co} \beta \max )}
$$

Where: $\cos \beta=\cos (b)$ at any point

$\cos \beta \max =\cos (b)$ at border

The pixel color is corrected by:

$$
I(i, j)=f \operatorname{cor}+I(i, j)
$$

An example of this final processing is shown on Figure 14b. The calculation of the average gray in a reduced image was 190 in the center and at the edges it was 148, 157, 145 and 147, with an average of 149 ; factor $190 / 149=1.275$. After applying the corrections the dark border is eliminated.

The Minimum (Min), Maximum (Max), Mean and Standard deviation (Stdev) of the Figure 14 are showed in table 2 . The global effect with the vignetting correction is to grow up the minimum and mean and reduce the standard deviation of the result image.

\begin{tabular}{ccccc}
\hline Basic Stats & Min & Max & Mean & Stdev \\
\hline Fig. 14(a) & 22 & 255 & 153.014712 & 34.643259 \\
\hline Fig. 14(b) & 77 & 255 & 178.653428 & 30.067176 \\
\hline
\end{tabular}

Table 2. Basic Statistics of Figure 14 


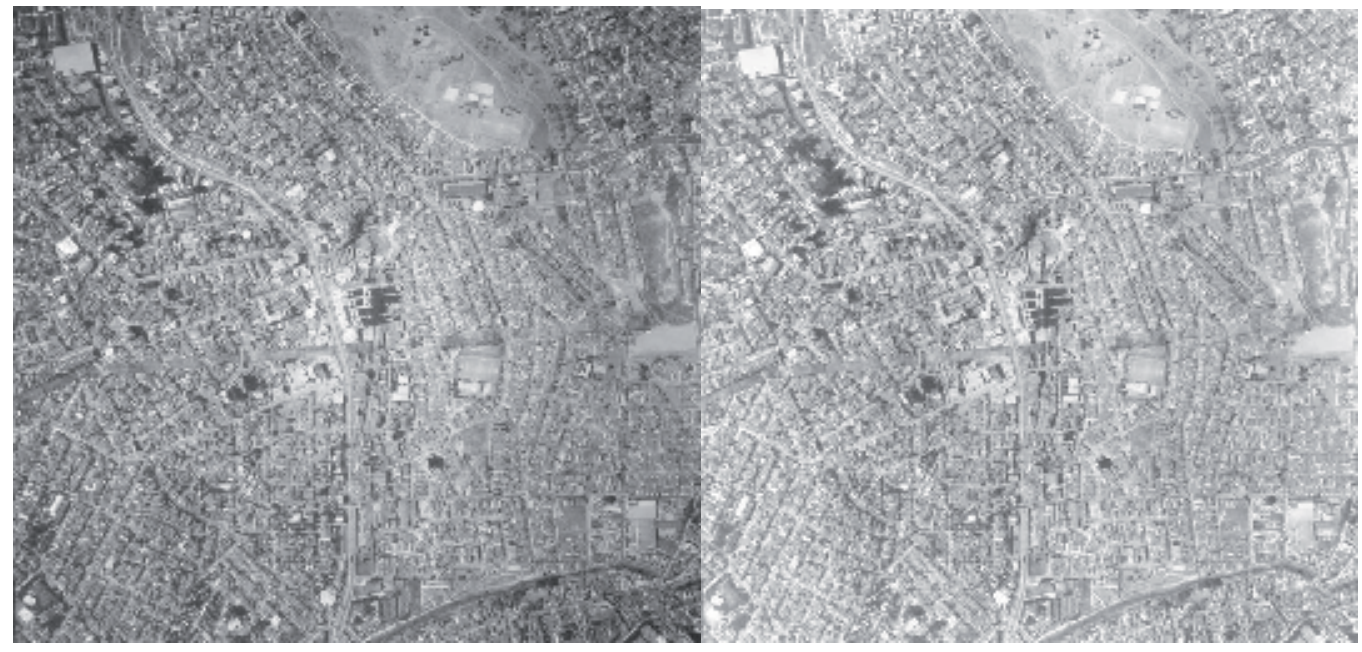

Fig. 14. Correction of vignetting effect of left image showed in the right.

For the color photograph (Figure 15a) the calculation by bands was performed: Red, center 200, edges 173, 155, 157 and, 161, average $=161$, factor $=1.238$; Green, center 184, edges 143, 142, 153 and 140, average $=144.5$, factor $=1.273$; Blue, Center 166 , edges 147, 141, 139 and 145 , average $=143$, factor $=1.161$. The result is shown on Figure 15b.

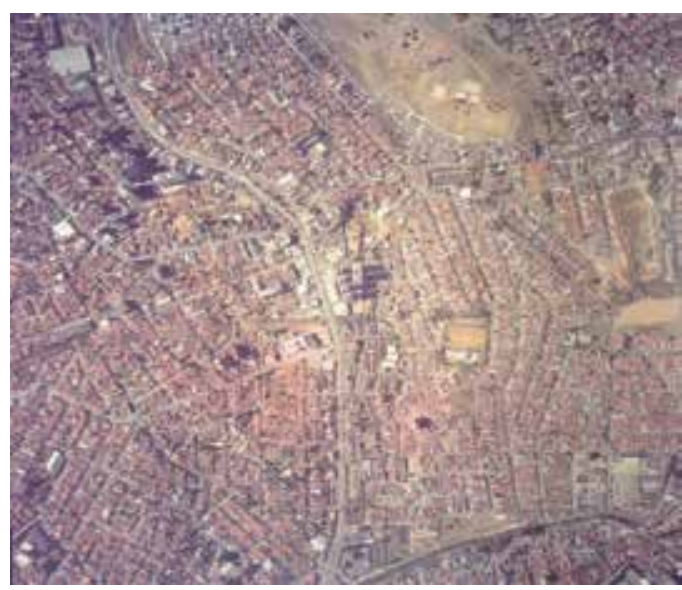

a)

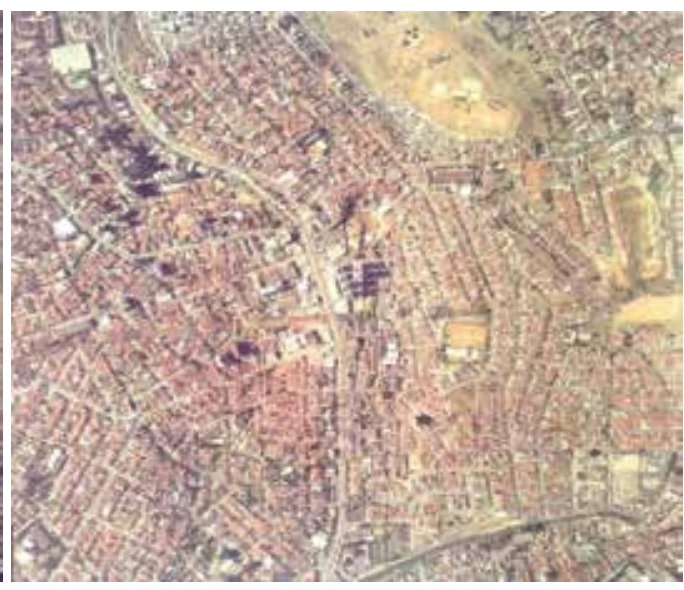

b)

Fig. 15. Vigneting in color photograph a) Original image, b) With Vignetting correction.

Another way of using fcor is to create a mask that can be applied to all images in a range of line flight, for example. Thus processing is faster, compared to methods of manipulating histograms, because you just add the masks of each band to the bands of the images. 


\subsection{Application of masks}

One of the processing results with an individually added mask to the RGB bands of Figure $16 \mathrm{a}$, is shown on Figure 16b. The very dark tonality in the inferior part of this figure was corrected. The colors of the darker parts are not well recuperated because they were saturated. This problem will be resolved in section 4.3.

The block balancing method was also applied to the same photograph and the result is shown on Figure 16c, where the mean tonality of the image is more uniform, but the inferior part is still very dark, aside from presenting the artifacts already shown on Figure 10

Table 2 shows the basic statistics of original image, image balanced with mask and with histogram blocks images of Figure 16. Observing the standard deviation (Table 3), it is possible to see that there is more variability of colors in original image and this effect is corrected in the other ones.

The method of mask addition, although it does not introduce artifacts and undesired color transitions, it is computationally more efficient, since the same mask can be used in many photographs taken at a certain interval of flight time, as in the strip (Figure 17). Moreover, the process of adding original image with the mask is quicker than the processing of the multiplications and divisions involved in other methods. For an image of 11,500 x 11,500 (pixels) and blocks of $100 \times 100$, the total time was 3min 40s with manipulation of histogram blocks, and only $1 \mathrm{~min} 10 \mathrm{~s}$ with the mask, on the same computer. The preparation time for the mask depends on the program used, but it can be totally automated.

\begin{tabular}{|c|c|c|}
\hline \multicolumn{1}{|c|}{ Original } & Wasic Statistics & \\
\hline Band: $\mathbf{R}$ & Wand: $\mathbf{R}$ & With block balancing \\
Min: 4.00 & Min: 4.00 & Band: $\mathrm{R}$ \\
Max: 255.00 & Max: 255.00 & Min: 4.00 \\
Mean: 139.31 & Mean: 149.52 & Max: 255.00 \\
Standard Deviation: & Standard Deviation: & Mean: 135.82 \\
58.64 & 34.82 & Standard Deviation: \\
\hline Band: G & Band: G & Band: G \\
Min: 0.00 & Min: 4.00 & Min: 0.00 \\
Max: 255.00 & Max: 255.00 & Max: 255.00 \\
Mean: 136.70 & Mean: 148.28 & Mean: 133.27 \\
Standard Deviation: & Standard Deviation: & Standard Deviation: \\
56.47 & 33.64 & 36.87 \\
\hline Band: B & Band: B & Band: B \\
Min: 0.00 & Min: 4.00 & Min: 0.00 \\
Max: 255.00 & Max: 255.00 & Max: 255.00 \\
Mean: 117.58 & Mean: 136.21 & Mean: 116.43 \\
Standard Deviation: & Standard Deviation: & Standard Deviation: \\
46.76 & 28.97 & 30.90 \\
\hline
\end{tabular}

Table 3. Basic Statistics 


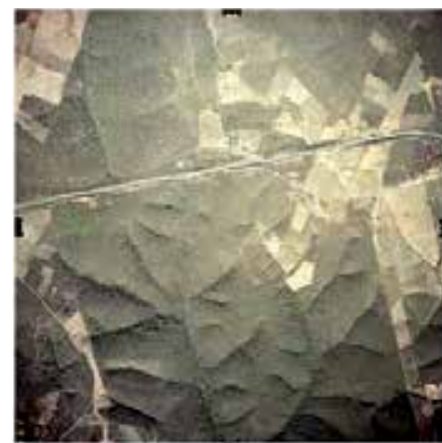

a) Original.

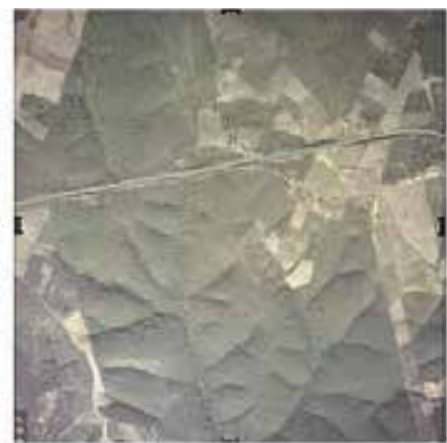

b) With mask.

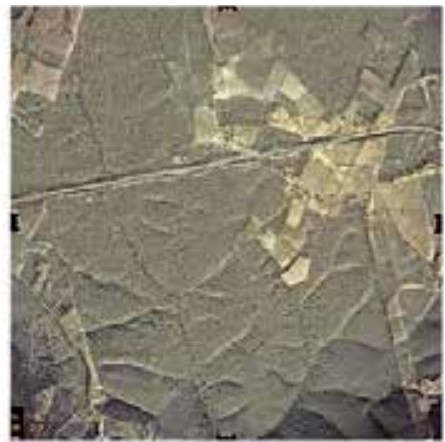

c) With histogram blocks

Fig. 16. Comparing processed images with mask and histogram manipulation

The basics statistics in Table 4 show that the strip with pre-processed images is brighter (Figure 17a) and it has lesser standard deviation than with rough image (Figure 17b). Qualitatively it can be seen in Figure 16.

\begin{tabular}{lc}
\hline Basic Statistics & \\
\hline With pre-processed images & With rough images \\
\hline Band: $\mathrm{R}$ & Band: $\mathrm{R}$ \\
Min: 57.00 & Min: 4.00 \\
Max: 255.00 & Max: 255.00 \\
Mean: 164.81 & Mean: 128.19 \\
Standard Deviation: & Standard Deviation: \\
33.79 & 46.73 \\
\hline Band: G & Band: G \\
Min: 57.00 & Min: 4.00 \\
Max: 255.00 & Max: 255.00 \\
Mean: 162.85 & Mean: 127.36 \\
Standard Deviation: & Standard Deviation: \\
31.68 & 45.96 \\
\hline Band: B & Band: B \\
Min: 51.00 & Min: 4.00 \\
Max: 255.00 & Max: 255.00 \\
Mean: 145.13 & Mean: 112.01 \\
Standard Deviation: & Standard Deviation: \\
27.23 & 35.74 \\
\hline
\end{tabular}

Table 4. Basic Statistics

In Figure 17a some difference of tonality can still be seen in the stick zones. This could be due to the use of only a single photograph for preparing the mask and the influence of the features of the land as the vegetation. Ongoing tests show that an average mask obtained from more than one photograph represents, with more accuracy, the combined illumination effects of the BRDF, haze and vignette. 


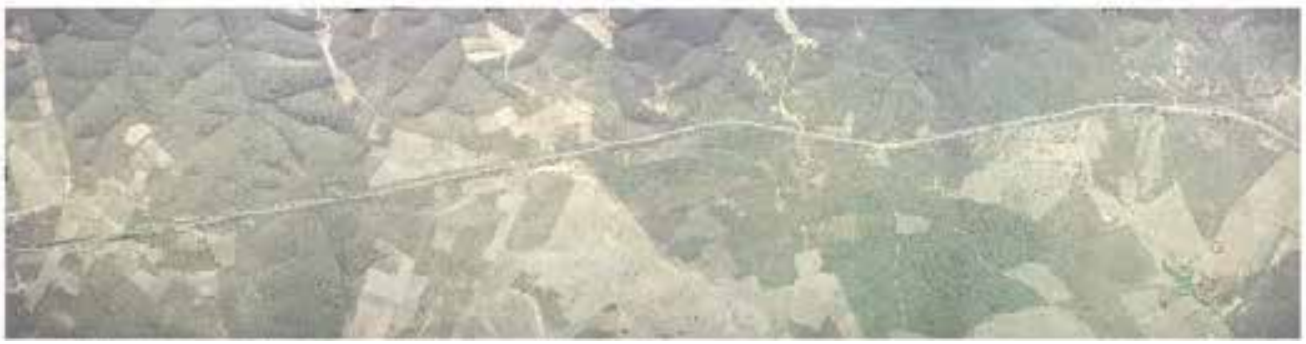

a)

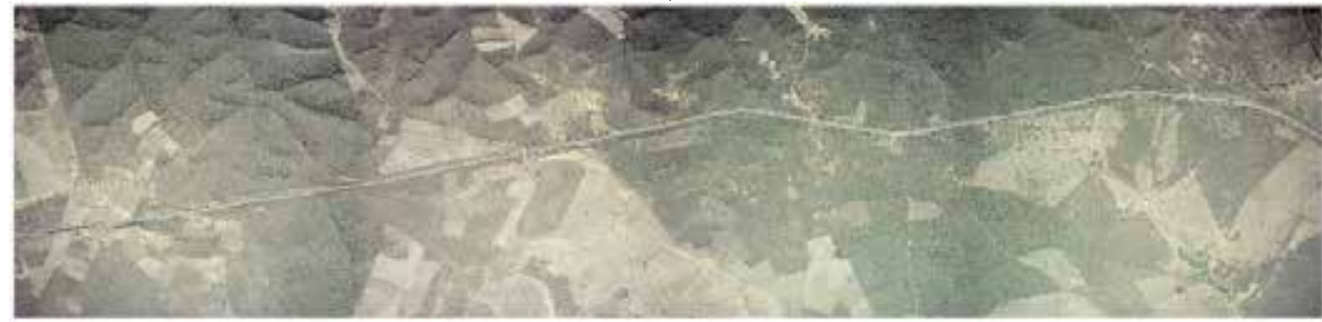

b)

Fig. 17. Example of a mosaic strip. a) Pre-processed with masks, b) With rough images. (Photograph: TOPOCART S/A).

However, the greater uniformity of tones observed in the images processed with masks is already enough to significantly improve the quality of a mosaic that is reprocessed with block balancing, as shown on Figure 18a, while on Figure 18b there are still areas with more shadows.

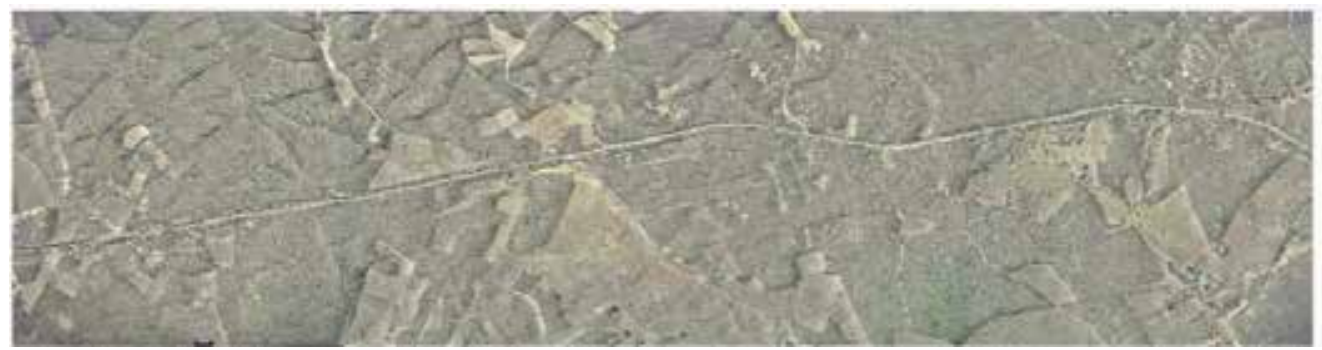

a)

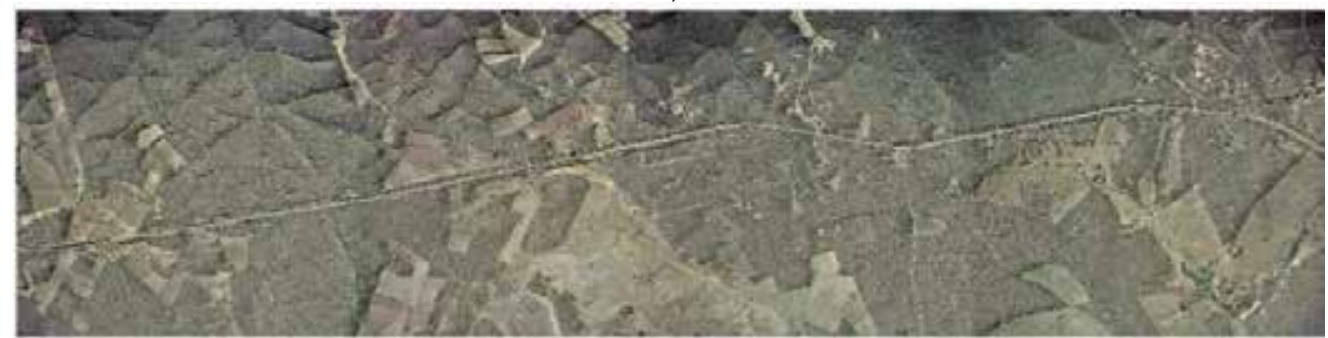

b)

Fig. 18. Mosaics processed with block balancing. a) Photographs pre-processed with masks, b) original photographs. (Photographs: TOPOCART S/A ). 
Various tests of mask use in colored aerial photographs were done with rural and urban sceneries in varied scales, and the following was observed:

- The correction works well for images with sceneries without significant variations of texture and tonality. If strong variations occur the masks must be made using an average of three or more photographs.

- The saturated colors in the borders of the original images are not recuperated and a pre processing is need.

- In case the mask is added to the HSV decomposition component V, the recomposed image keeps the tone of the areas of more altered saturated hues (alteration of hue), than when processing with $R G B$ bands.

\subsection{Correction of full frame}

\subsubsection{Using Kries method}

The general color aspects of an image should be adjusted to a color standard of another image using the Von Kries hypothesis. Similar procedure can also be applied using only color band adjustment present in most graphic processing software, but some practical experience from the operator is necessary.

The image for this kind of color correction must not be saturated. The saturation can be indicated by the presence of too many pixels (peak) at the ends of the histogram, in one or more bands. The histogram of image in Figure 19 shows that it has not color saturation.

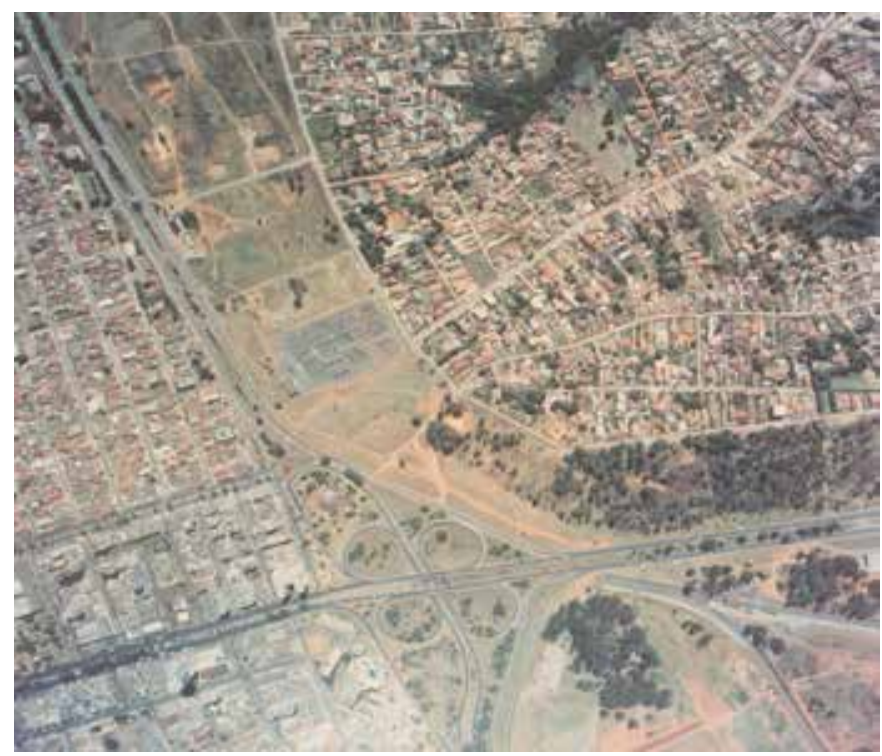

a)

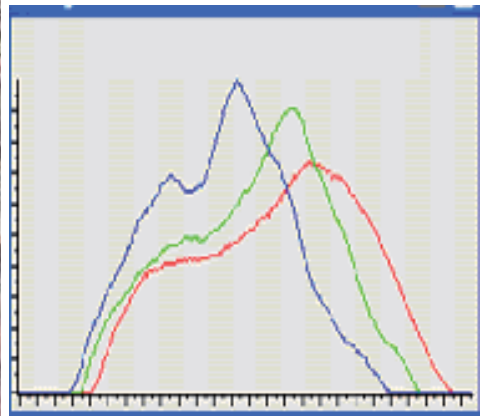

b)

Fig. 19. a) Image example without color saturation, b) Histograms of the RGB bands.

The image in figure 19 was processed with Kries method and using color cast of figure 20 resulting in image showed in figure 21 . The coefficients were $K R=1.0759, K G=1.1751$, $\mathrm{KB}=1.1451$ 


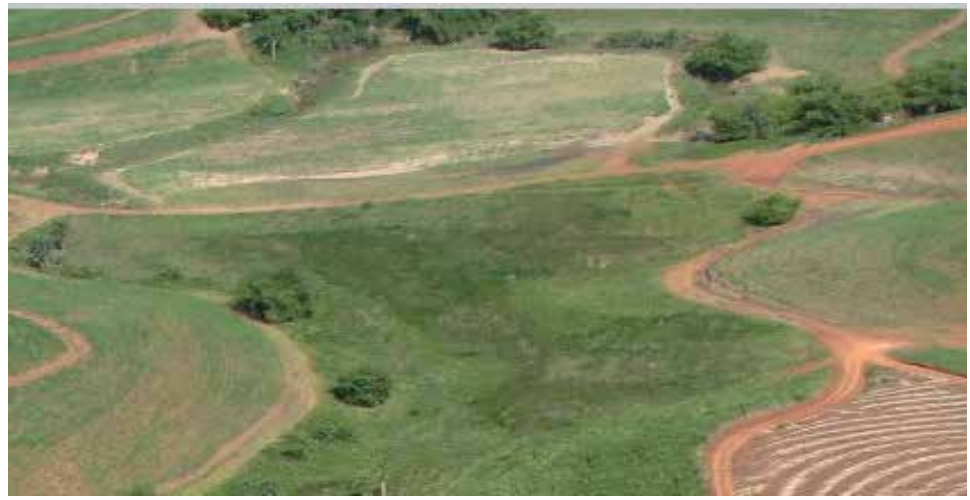

Fig. 20. Image with a color cast desired.

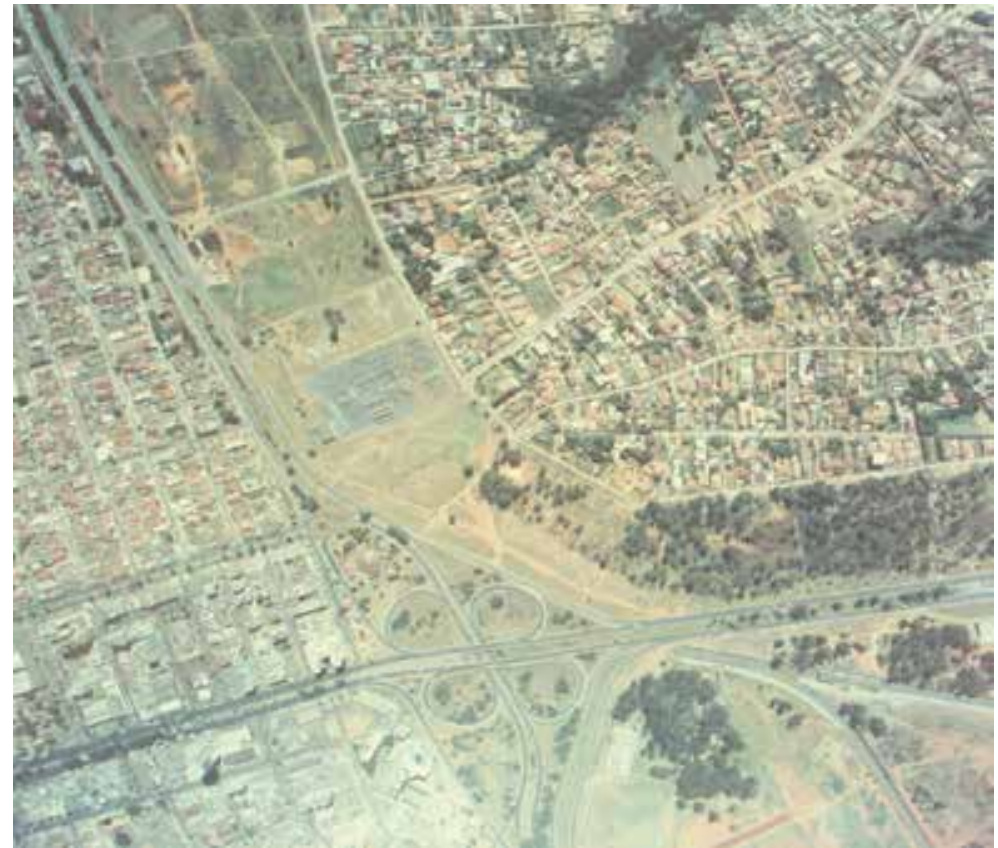

Fig. 21. Image processed with Kries method.

An example of image with color saturation in the red band is in figure 15a (histogram in figure 22a). The practical solution to eliminate the saturation is to manipulate the luminance or intensity, in order to remove the peak value occurs at 255. In this example firstly was reduced the intensity range using gamma correction setting it to 0.60 and then all the intensity levels was reduced in 10 units. These values were determined by trial and error, but it can also be used image statistics to see the saturation and the need intensity shift. The final result of the histogram of red band is shown in Figure 22b. 


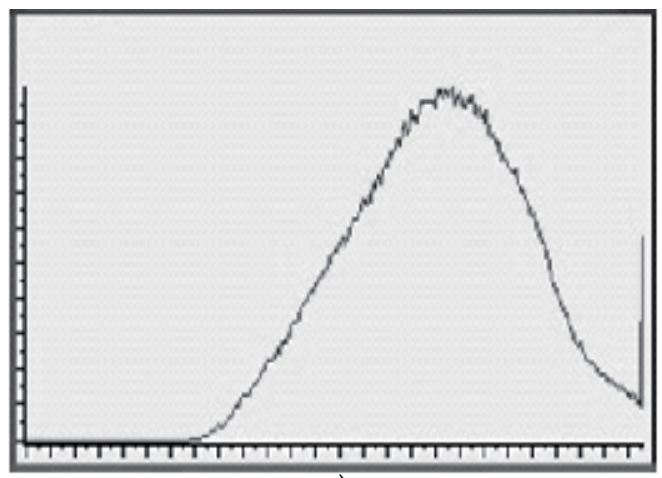

a)

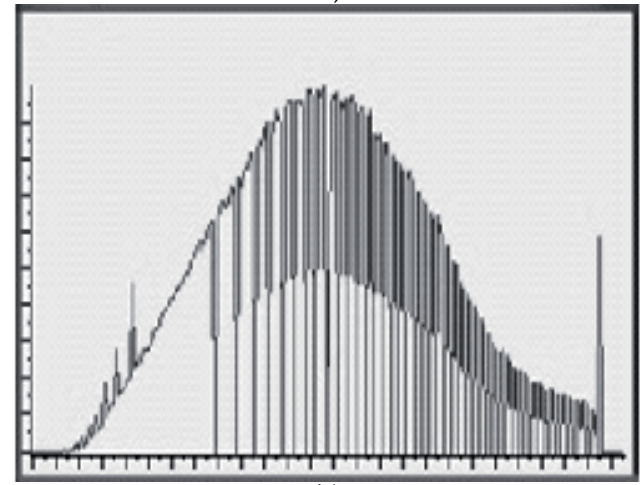

b)

Fig. 22. Histogram of red band figure 15a. a) original saturated, b) After change.

The reconstruction of the RGB image using the new red band results in the image of the figure 23 with a ton greener more real.

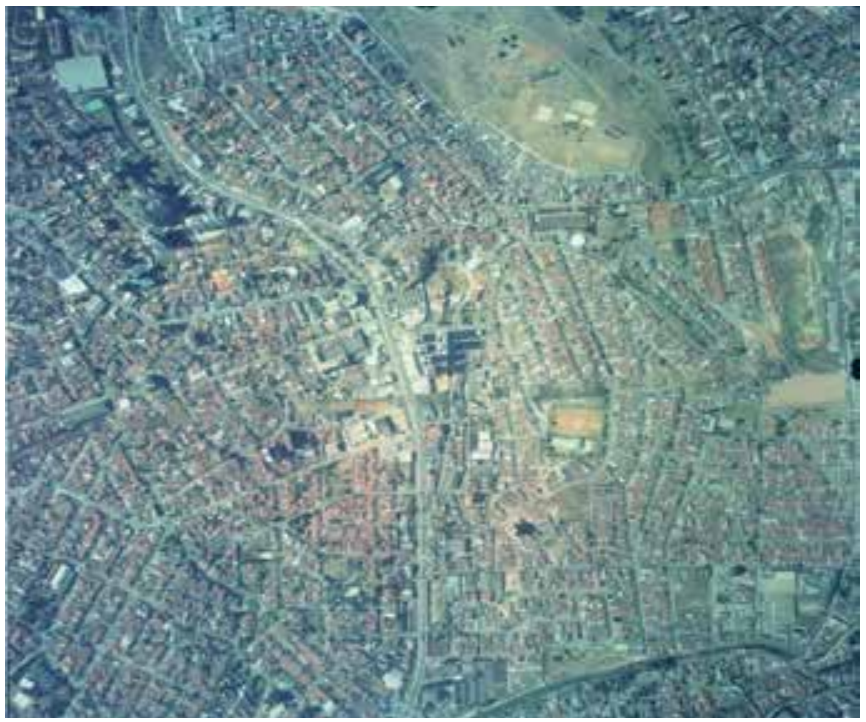

Fig. 23. Image of figure 15a with de-saturation of Red band 


\subsubsection{Using histogram matching}

The use of the histogram matching (section 3.4) can also change the color cast of an image. The figure $24 \mathrm{~b}$ shows the histograms of the image with strong reddish cast to be changed, the figure 24a shows the histograms of the image 21 (template) and figure $24 \mathrm{c}$ the final matching histogram. The figure 25 shows the new image with the new histograms.

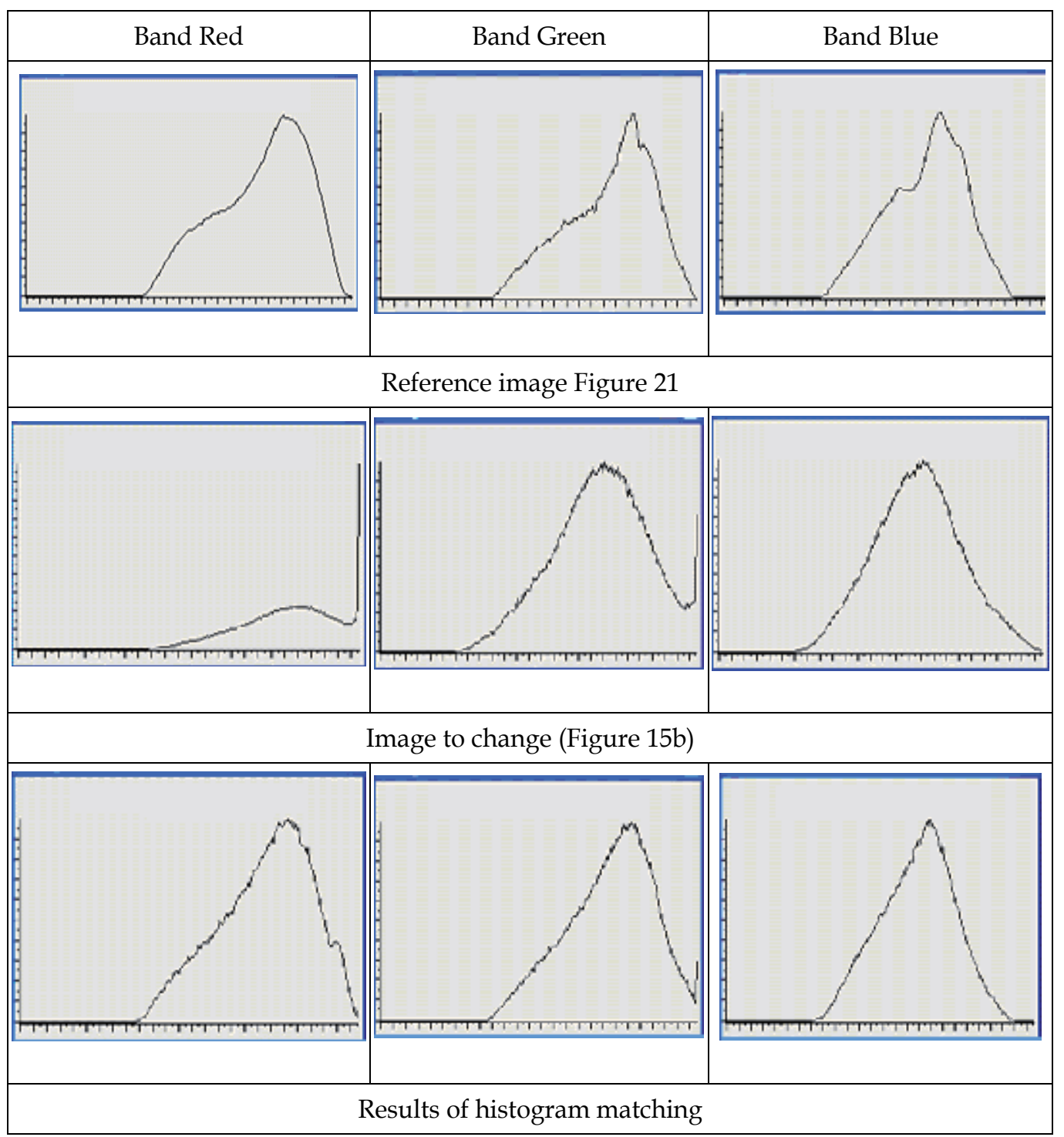

Fig. 24. Histogram matching a) of image template, b) of image to be changed, c) result of the matching 


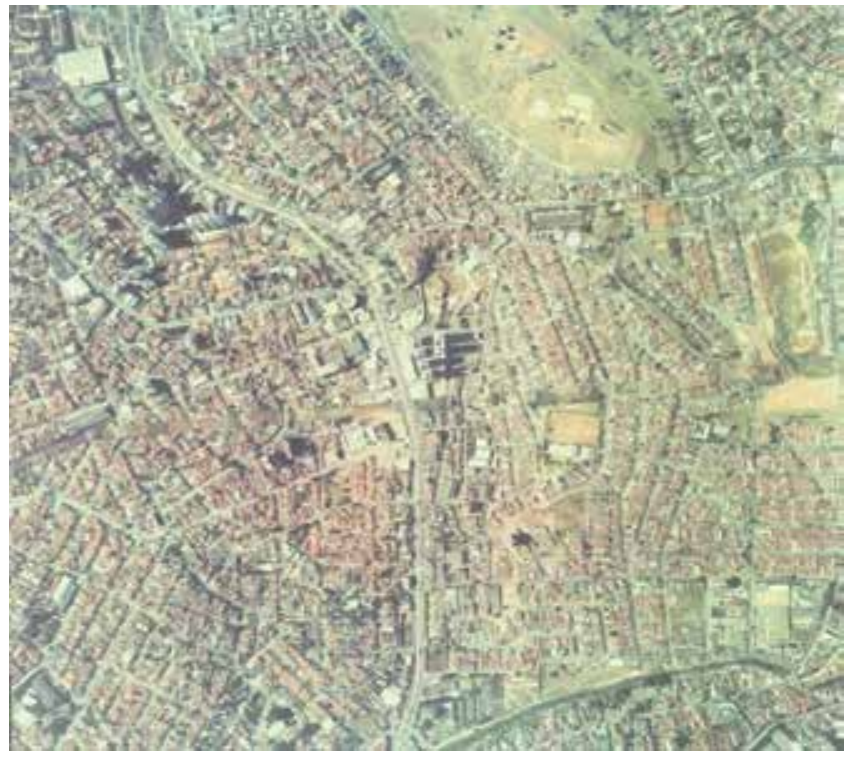

Fig. 25. Final Image of histogram matching on image 15b

The image of figure $15 \mathrm{~b}$ was also processed with the Kries method using as color standard the same figure 21. The final corrected image (Figure 26) looks similar to image above but more greenish and their histograms have some differences (Figure 27).

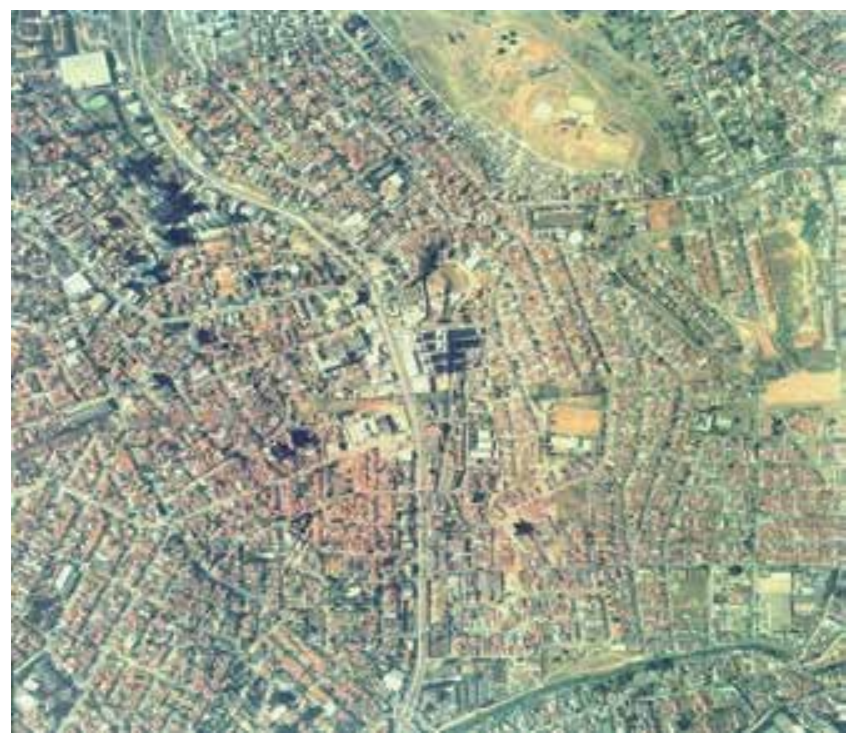

Fig. 26. Using Kries method in image 14b 


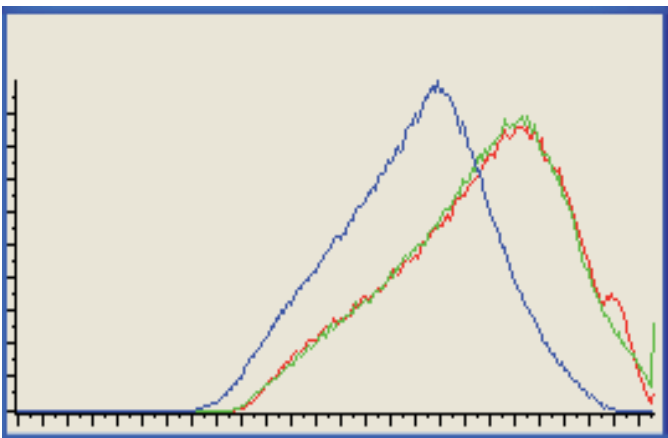

a)

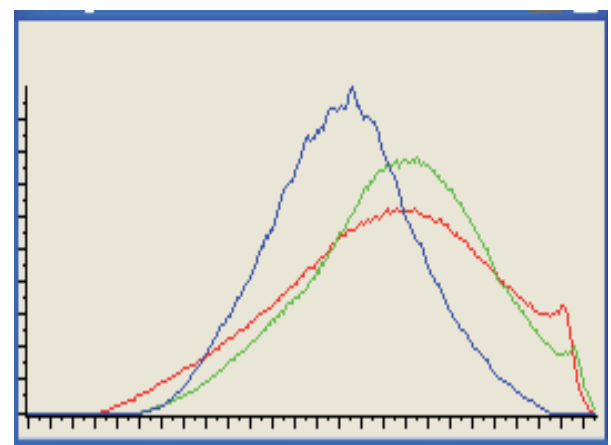

b)

Fig. 27. Histograms after restoratios a) of figure 25 by match, b) of figure 26 by Kries.

In a general way these two methods of changing the color cast have minor computational cost than the homogenization of histogram blocks. The best choice and the efficiency in practical applications will be defined by the software implementation. In some cases only one method would give the desired result and in others a combination of both histogram matching and the Kries method would be better fitting.

\section{Conclusions}

This chapter discusses first, the causes of undesirable color changes in aerial photographs due to atmospheric factors and geometric factors of imaging, like the haze effect, position of the sun and aperture angle of cameras, and in second place some procedures for restorations of those colors.

The combination of the vignetting effect, and of the backscattering of light in haze, results in a non-uniform intensity on photographic frame with an irregular format, different for each photogrammetric flying, so that is not possible to correct it using only the simple model based in the cosine law.

The methods of color restoration in aerial photographs using mask, manipulation of histograms and the Kries hypothesis are discussed and applied with several examples. These methods have minor computational cost than the homogenization among histogram blocks used in commercial softwares.

\section{References}

Ashikhmin, M. (2002). A Tone Mapping Algorithm for High Contrast Images. In: Eurographics Workshop on Rendering. Paul Debevec and Simon Gibson (Editors) Proceedings of the 13th Eurographics Workshop on Rendering Techniques, Pisa, Italy, June 26-28, 2002.

Asrar, Ghassem (Ed). (1989). Theory and Applications of Optical Remote Sensing. New York: John Wiley \& Sons. 734 P.

Beisl, U; Woodhouse, N. (2004). Correction Of Atmospheric And Bidirectional Effects In Multi-Spectral Ads40 Images For Mapping Purposes. The International Archives Of 
The Photogrammetry, Remote Sensing and Spatial Information Sciences. V. 34 Part XXX. Isprs Instambul Cd-Rom.

Drago, F; Myszkowsky, K; Annen, T; Chiba, N. (2003). Adaptative Logarithmic Mapping for Displaying High Contrast Scenes. Eurographics 2003. V. 22, N. 3.

Fiete, Robert D. (2004). Elements of Photogrammetric Optics. In: Manual Of Photogrammetry (5th Edition). Bethesda, Maryland, USA: American Society For Photogrammetry And Remote Sensing.

Gonçalves, Glauber A. (2006). Detecção Automática de Alterações na Cartografia Cadastral com Base em Imagens de Câmaras Digitais. Doctoral Thesis. Curitiba: Curso de PósGraduação em Ciências Geodésica, Universidade Federal do Paraná.

Hall, R. (1954). The Effect of Haze and High Solar Altitude on the Density of Air Survey Negatives. Photogrammetric Record. V. 1. N. 4. P. 20-37.

Homma, Kohzo; Yamamoto Hiromichi; Iwata, Yoshitaka. (2000). A Study on Image Restoration for Airborne Cameras. In: The International Archives Of The Photogrammetry, Remote Sensing. V. 33 Part B7. Proceedings XiXth Congress Isprs Amsterdam Cd-Rom.

Kraus, Karl. (1992) Photogrammetr, Volume 1, Fundamentals and Standard Processes. Bonn: Dümmler. 397 P.

Kraus, Karl.(1997). Photogrammetry, Volume 2, Adavanced Methods and Applications.. Bonn: Dümmler. 466 P.

Lamparelli, Rubens Augusto C; Rocha, Jansle V; Jaime, Andrés L. G. (2004). Correção Radiométrica de Imagens Digitais Aéreas para o Efeito Vinhete. Revista Brasileira de Cartografia. N. 56. V. 01. P. 65-74.

Li, Zh J; Zhang, Z.X; Zhang J. Q. (2004a) Dodging In Photogrammetry And Remote Sensing. In: The International Archives of the Photogrammetry, Remote Sensing and Spatial Information Sciences. V. 34 Part B5. Proceedings XIXth Congress Instanbul.

Li, Yan; Sasagawa, T; Gong, Peng. (2004b). A System Of The Shadow Detection And Shadow Removal For High Resolution City Aerial Photo. In: International Archives Of Photogrammetry And Remote Sensing and Spatial Information Sciences. V.35 Part B3. Proceedings XIXth Congress Instanbul.

Mcglone, J. C.(Ed). (2004). Manual Of Photogrammetry (5th Edition). Bethesda, Maryland, Eua: American Society for Photogrammetry and Remote Sensing. 1151 P.

Nóbrega, Rodrigo A. A; Quintanilha José A. (2004). Comparative Analysis Of Automatic Digital Image Balancing And Standard Histogram Enhancement Techniques In Remote Sensing Imagery. Revista Brasileira de Cartografia. N. 56. V. 01. P. 55-64.

Paparodis, N; Souchou, J. P.; Martinoty, G; Deseilliny, M. P..(2006). High-End Aerial Digital Cameras and their Impact on the Automation and Quality of the Production Workflow. Isprs Journal of Photogrammetry and Remote Sensing. V. 60, N. 6. P. 400-412.

Reinhard, E; Stark, M; Shirley P; Ferwerda J. (2002). Photographic Tone Reproduction For Digital Images. Preccedins of The Siggraph 2002.

Schowengerdt, R. A. (1997). Spectral Transforms. In: Remote Sensing Models And Methods For Image Processing. 2 Ed. San Diego, Ca, Cap. 5, P. 202-219.

Silva, D. C., Candeias, A. L. B (2009). Causas da Iluminação não Uniforme em Fotografias Aéreas Coloridas. Revista Brasileira de Cartografia. v.61, p.105 - 115. 
Silva, D. C; Candeias, A. L. B. (2008). Restoration of Non Uniform Illumination in Color Aerial Photographs. Boletim de Ciências Geodésicas. Curitiba: Ufpr. V.14, N.3.

Slater, Philip N. (1980). Remote Sensing, Optics and Optical Systems. Massachusetts: Addison-Wesley Publishing. Il. 575 P..

Slater, Philip N. (Ed). (1983). Photographic Systems for Remote Sensing (Chap 6). In: Manual of Remote Sensing 2nd Edition. V.1. American Society of Photogrametry.

Tuominen, Sakari; Pekkarinen, Anssi. (2004). Local Radiometric Correction of Digital Aerial Photographs for Multi Source Forest Inventory. Remote Sensing of Environment. N. 89, P. 72-82.

Wu, Xiaoliang; Campbell, Norm. (2004). A Colour-Balancing Method and Its Applications. In: The 12th Australasian Remote Sensing and Photogrammetric Conference Proceedings. Fremantle, Western Australia, 2004.

Wyszecki, G; Stiles (1982)., W.S. Color Science Concepts and Methods, Quantitative data and Formulae. 2th edition. John Wiley \& Sons. 


\title{
High-Quality Seamless Panoramic Images
}

\author{
Jaechoon Chon, Jimmy Wang, Tom Slankard and John Ristevski \\ Earthmine Inc., Berkeley, \\ USA
}

\section{Introduction}

Image mosaicing is the creation of a larger image by stitching together smaller images. Image mosaicing has many different applications, such as satellite imagery mosaics (Chon et al., 2010,) the creation of virtual reality environments (Szeliski, 1996; Chon et al., 2007; Brown and Lowe, 2007,) medical image mosaics (Chou et al., 1997,) and video compression (Irani et al., 1995; Irani and Anandan, 1998; Kumar et al., 1995; Lee et al., 1997; Teodosio and Bender, 1993).

Image mosaicing has four steps: 1) estimating relative pose among smaller images to project on a plane or specific defined surfaces, 2) projecting those images on the surface, 3) correcting photometric parameters among projecting images, and 4) blending overlapping images.

The first step has two categories: 2D plane images (For example, scanned maps in sections and orthoimages) based methods and perspective image based methods. Orthoimages are generated by correcting each image pixel from a perspective image with a help of a Digital Elevation Model (DEM,) produced by a stereo camera system, a laser/radar aerial system or obtained of photogrammetric works. To align the images with the DEM, we need to estimate absolute orientation with ground control points (GCP.) In the case of perspective images, we need to estimate relative pose using homography, affine transformation, colinear conditions, and coplanner condition with feature correspondences. (Hartley and Zisserman \& Books, 2003; McGlone et al. \& Books, 2004; Szeliski \& Books, 2011)

The second step projects all images onto a specific defined surface, such as 2D plane, cylinder (Chen, 1995; Szeliski, 1996), sphere (Coorg and Teller, 2000; Szeliski and Shum, 1997), and multiple projection planes (Chon et al., 2007.) In the case of cylinder and sphere, we assume that images are captured by fixing the position of a camera and rotating it at the position. And all images are projecting onto a cylinder or a sphere surface.

In the third step, we have to balance photometric parameters among projected images because exposure affects the photometric parameters of each image. At the last step, we have to create one image from multiple overlapping images.

Ideally each sample (pixel) along a ray would have the same intensity in every image that it intersects, but in reality this is not the case. Even after gain compensation some image edges are still visible due to a number of un-modeled effects, such as vignetting (the decrease of intensity towards the edge of the image,) parallax effects due to unwanted motion of the optical center, registration errors due to an incorrect or approximate camera model, radial distortion, and so on. 
Because of this, a good blending strategy is important (Brown and Lowe, 2007; Goldman, 2011; Kim and Pollefeys, 2008; Zomet et al., 2006.) In particular, applying seam-line detection algorithms before applying blending algorithms is an effective strategy when using image data that has parallax effects, because a seam-line detected by optimal path finding algorithms passes equal-depth pixels or similar colors pixels overlapping images.

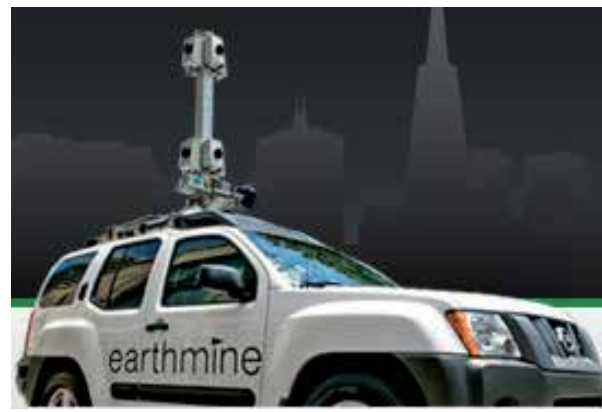

a) Vehicle system

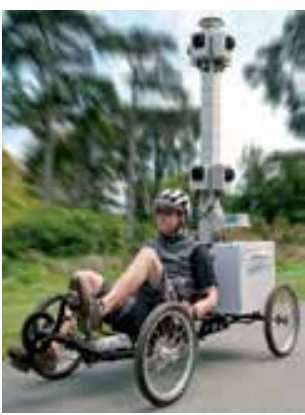

b) Quad cycle system
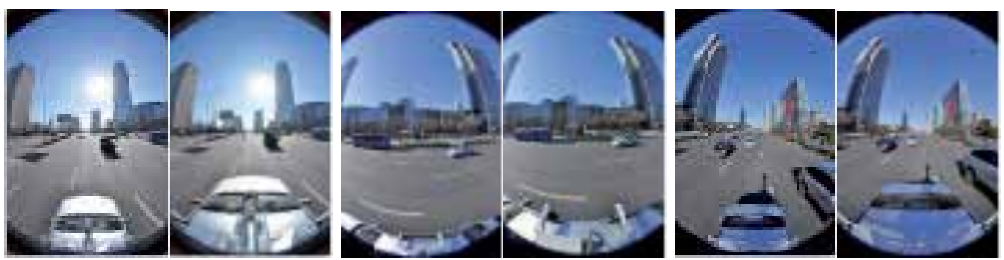

c) 4 stereo fish-eye images
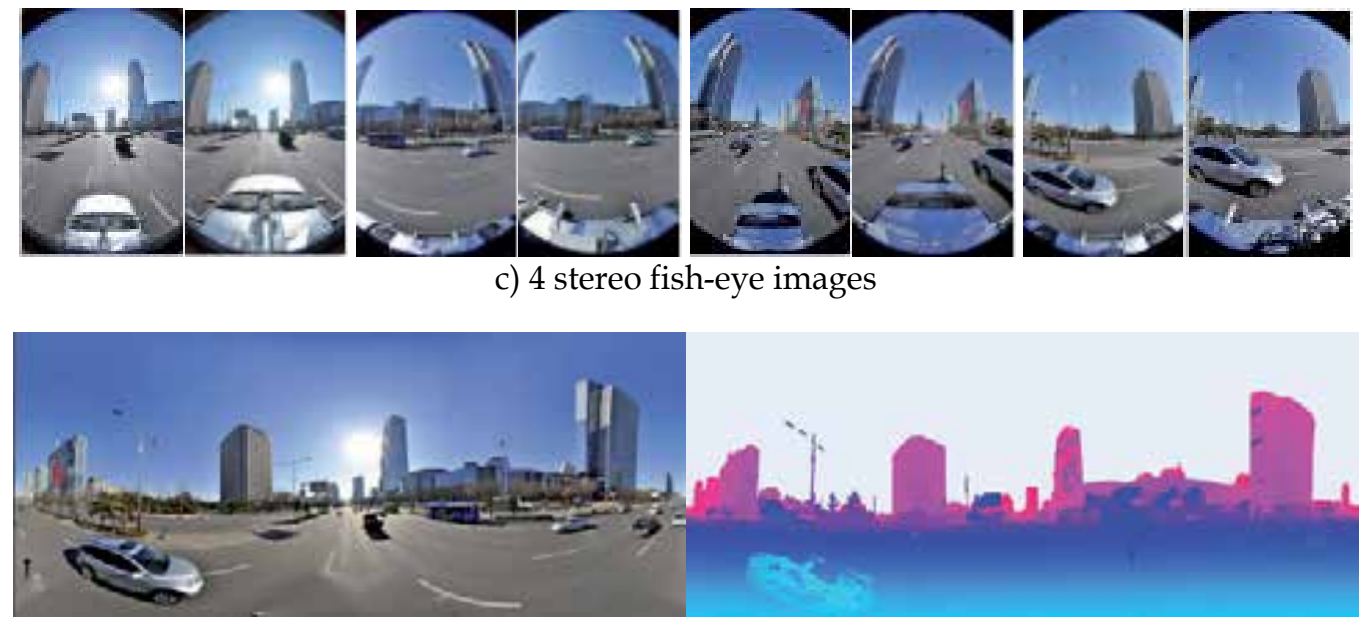

d) Image and 3D range panoramas

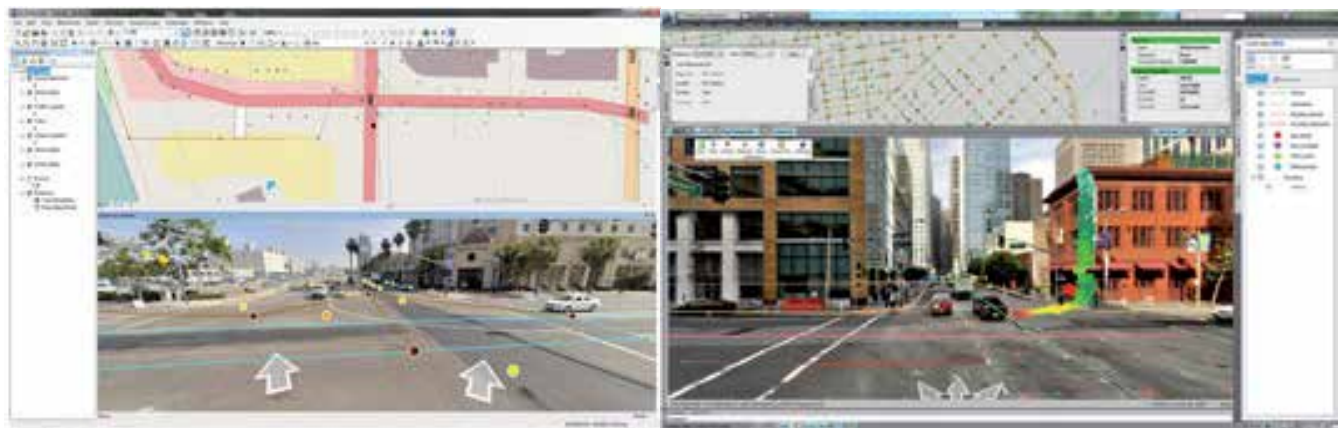

e) Applications of ArcGIS and AutoCAD Map3D

Fig. 1. EARTHMINE Inc.'s 3D mobile mapping systems, 3D panorama generated from eight fish-eye images captured using the system, and its applications. 
In this chapter, we introduce luminance balancing using gamma correction, Dijkstra's algorithm for seam-line detection, our proposed fast color blending algorithm on seam-lines, and color balance and saturation implemented on EARTHMINE Inc.'s mobile mapping system (MMS) that generates 3D panoramic images as shown in Fig 1.

\section{Color balance}

A major challenge in merging multiple shots into a single panorama is that each individual image experiences its own exposure setting, even if each camera (when there are multiple cameras) is color calibrated. A common scenario that may cause such discrepancy is when the shots are taken, some cameras face towards the light source (e.g. the sun), and others face away from the light source. Due to the different exposure to the physical light source, some images will be brighter than others. Merging these images together inevitably causes a visible seam at the merging points.

To overcome this problem, we describe an algorithm that detects the differences in the luminance and chrominance in each image and attempts to equalize such differences. First, we assume that there exist overlapping regions between neighboring images. For the set of images, we assume there exist a single correction factor per channel (one luminance channel that governs image intensity and two chrominance channels that governs the color of the image) per image such that the luminance/chrominance of the overlapping areas match up.

The ideal approach would be to find corresponding pixels between the overlapping areas and find the optimal correction factors using these correspondences. Specifically, we can write the optimization process in the following form

$$
\arg \min \left[\sum_{p}\left(\gamma_{n} I_{n p}-\gamma_{m} I_{m p}\right)^{2}\right]
$$

where $I_{n p}$ and $I_{m p}$ are the $p^{\text {th }}$ corresponding channel value for image $I_{n}$ and $I_{m}$ respectively; $\gamma_{n}$ and $\gamma_{m}$ are the correction factor for image $n$ and $m$. A simple illustration is shown in figure 2.1.
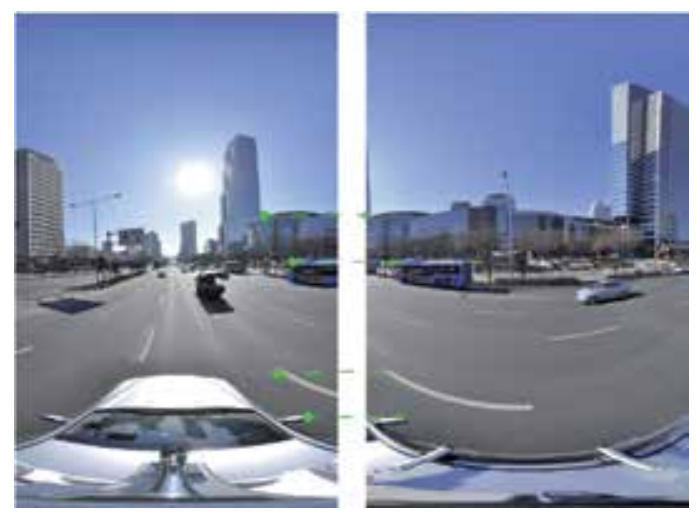

Fig. 2.1. An illustration of a subset of the correspondences between two images in the overlapped region. 
However, finding pixel correspondences between pairs of images is non-trivial and most currently available algorithms such as SIFT (Lowe, 2004) are very computationally expensive. For applications that have limited processing power (e.g. image stitching on mobile platforms) or real time processing requirements, such approach is inadequate.

Brown and Lowe (2007) and Xiong and Pulli (2010) pointed out that because luminance/chrominance is a global entity, instead of using pixel correspondences, we can approximate them using the mean luminance / chrominance value between the overlapping region. $\mathrm{Eq}(2.1)$ thus becomes

$$
\arg \min \left[\left(\gamma_{n} E\left[I_{n}\right]-\gamma_{m} E\left[I_{m}\right]\right)^{2}\right]
$$

where E[In] and E[Im] denotes the average luminance / chrominance values of the overlapped image regions from image $\mathrm{n}$ and $\mathrm{m}$ respectively.

Note that there is a trivial solution to $\mathrm{Eq}(2.2)$, where when the $\gamma$ s are set to 0, the objective function is at its minimum. To prevent this, we add a regularization term in the form of 1 $\gamma$, such that this term is non-zero when $\gamma$ is 0 . In addition, this term prevents the luminance / chrominance from changing too much, which is often desired. The objective function thus becomes

$$
\underset{\gamma_{n}, \gamma_{m}}{\arg \min }\left[\omega_{d}\left(\gamma_{n} E\left[I_{n}\right]-\gamma_{m} E\left[I_{m}\right]\right)^{2}+\omega_{p}\left(1-\gamma_{n}\right)^{2}+\omega_{p}\left(1-\gamma_{m}\right)^{2}\right]
$$

where $w_{d}$ and $w_{p}$ are weights for the two terms.

The human visual system perceives brightness according to the Weber-Fechner law where the perceived brightness is not a linear function of the physical brightness but rather follows a $\log$ function (red line in figure 2.1; blue line indicates a linear response).

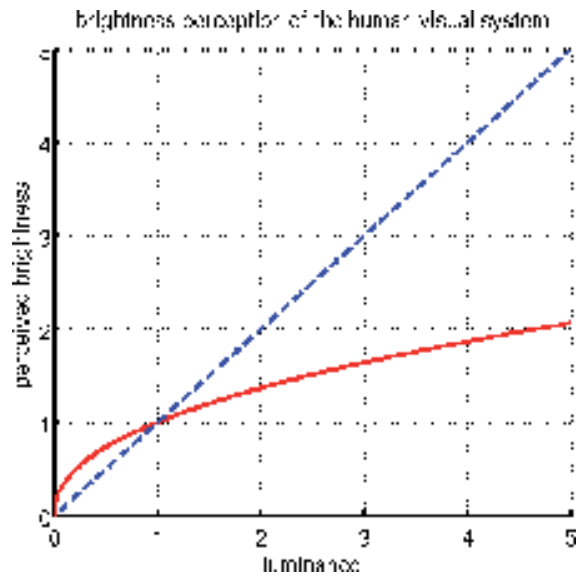

Fig. 2.2. An illustration of Weber-Fechner law of brightness perception.

To account for this effect, we linearize the luminance channel with a power function such that the brightness perception curve is linear (some cameras have this linearization in the post-processing step. If so this step can be omitted). The objective function for the luminance channel becomes 


$$
\underset{\gamma_{n}, \gamma_{m}}{\arg \min }\left[\omega_{d}\left(\gamma_{n} \frac{1}{P} \sum_{P}\left(I_{n p}\right)^{2.2}-\gamma_{m} \frac{1}{P} \sum_{P}\left(I_{m p}\right)^{2.2}\right)^{2}+\omega_{p}\left(1-\gamma_{n}\right)^{2}+\omega_{p}\left(1-\gamma_{m}\right)^{2}\right]
$$

where $P$ is the number of overlapping pixels.

We used a correction factor of 2.2 according to common practice (Poynton 2002). Xiong and Pulli (2009) also used a very similar objective. The final objective over all the images is thus

$$
\underset{\gamma_{1}, \cdots, \gamma_{\kappa}}{\arg \min }\left[\sum_{k=0}^{\kappa}\left(\omega_{d}\left(\gamma_{k} E\left[I_{k}^{l}\right]-\gamma_{k-1} E\left[I_{k-1}^{l}\right]\right)^{2}+\omega_{p}\left(1-\gamma_{k}\right)^{2}\right)\right]
$$

In the above equation, we assume that there are only two overlapping images (left and right) for a given image. While this is often the typical scenario, it is straightforward to generalize to any arbitrary amount of images. This objective function thus can be optimized with any of-the-shelf optimization package.

\section{Seam line detection}

To create a seamless panorama from a multiple stereo fish-eye camera system, with a system like the EARTHMINE Inc, is necessary an optimal seam-line detection algorithm (see Figs. 1(c) and 1(d).)

Dijkstra's algorithm to find an optimal path within a cost space (Bellman, 1957; Dijkstra, 1959.) A seam-line can be detect by using the Dijkstra's algorithm with a cost map built by a cross correlation between two overlapping images (Milgram, 1975, 1977; Davis, 1998; Efros and Freeman, 2001.)

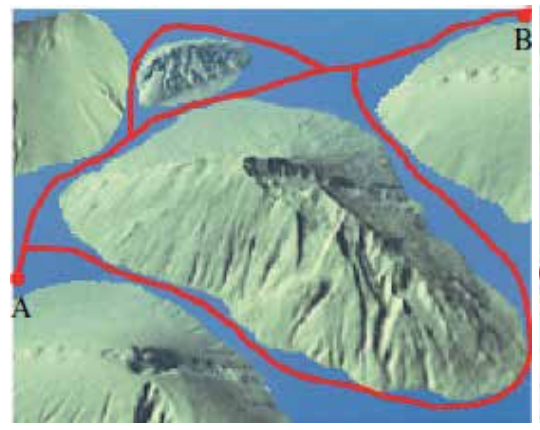

a)

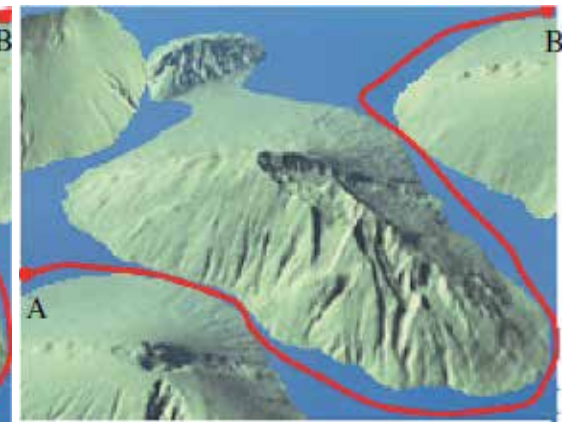

b)

Fig. 3.1. Chon et al. (2010) proposed seam-line algorithm explained with the waterway on the terrain structure. a) High water level creates many possible paths between two points $\mathrm{A}$ and B. b) Lowered water level makes the maximum cost smaller.

Chon et al. (2010) proposed a novel algorithm for selecting a seam-line that at first identifies a subarea within two overlapping images such that in the subarea, at least a seam line exists and the maximal mismatch score in this subarea is minimized. Let the degree of mismatching of pixels between two images on the overlapped region form a cost field, which can be expressed in a 3D plot. If a threshold is set with all cost values under the 
threshold filled with "water", then the 3D plot will look like the ones in Fig. 4, where Fig. 3.1(a) uses a larger threshold. The plots show some "water ways" between points A and B, and the threshold defines the water level. Fig. 3.1(b) shows that the allowed path is not near the shortest one. The technique then further applies Dijkstra's algorithm to find an optimal path within the restricted subspace. In this optimization phase, a cost conversion is applied to make a higher cost (the mismatch score) larger. This enables the search to find a possibly longer seam-line with less highly mismatched pairs.

Kerscher (2001) proposed a method called the "twin snake algorithm" to detect seam-lines. The algorithm starts with two initial vertical lines as control points of the snakes on the overlapping images. The snakes have two energy terms which are internal and external energies in general (Kass et al., 1987; Leymarie and Levine, 1993; Tiilikainen, 2007; Williams and Shah, 1990). The sum of the mismatching values on those lines and the relationship between neighbor control points are called internal energy and external energy, respectively. The twin snake algorithm builds an energy function $E_{\text {snake }}^{*}$ from four terms: the internal term $E_{\text {int }}$, the photometric term $E_{\text {pho }}$, and the external force $E_{\text {ext }}$. The energy is calculated for each vertex $v(s)$ and integrated over the whole length of the snake:

$$
E_{\text {snake }}^{*}=\int_{0}^{1} E_{\text {snake }}(v(s)) d s=\int_{0}^{1}\left(E_{\text {int }}(v(s))+E_{\text {pho }}(v(s))+E_{\text {ext }}(v(s))\right) d s
$$

The internal energy $E_{\text {int }}$ tries to preserve a smooth shape for the curve. Photometric energy $E_{\text {pho }}$ usually evaluates edge strength or similar measures in the examined image and tries to pull the snake to salient image features. External energy $E_{\text {ext }}$ can be introduced by user interaction and are responsible for globally controlling and guiding the snake evolution (Kerscher, 2001.) The curve with minimum energy as shown in Fig. 3.2 is determined to be the optimal seam line.

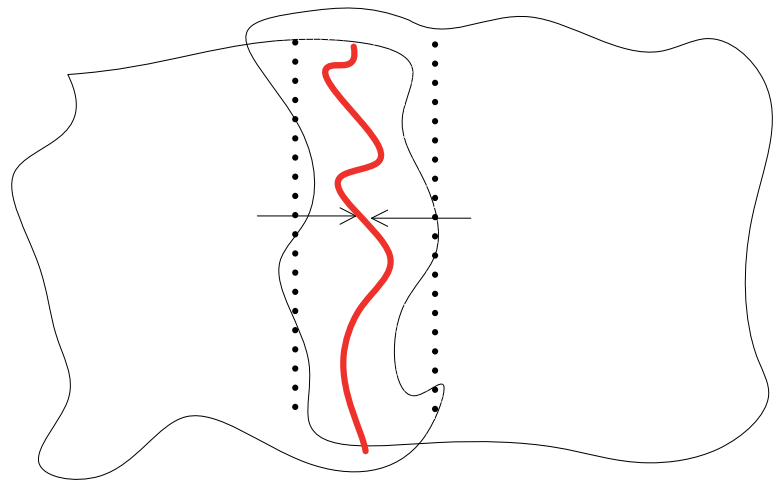

Fig. 3.2. Initial vertical lines as control points (dotted line) and a detected seam line (curved line).

This algorithm cannot completely overcome the local minima problem, and it requires a high computation load. Even though Chon et al. (2010) avoid the local minima problem, it requires a high computation cost as well because of finding the best threshold. 


\subsection{Cost space}

We adopts Dijkstra's algorithm to find an optimal seam-line on a cost space built by using the normalized cross correlation (NCC) between two overlapping images for pixel $(u, v)$ (Lewis, 1995). The NCC has been widely using image matching for stereo matching, feature tracking, etc.

$$
\operatorname{NCC}(u, v)=\frac{\sum_{i=u-w / 2}^{u+w / 2} \sum_{j=v-h / 2}^{v+h / 2}\left(I(i, j)-I_{u v}\right)\left(I^{\prime}(i, j)-I_{u v}^{\prime}\right)}{\sqrt{\sum_{i=u-w / 2}^{u+w / 2} \sum_{j=v-h / 2}^{v+h / 2}\left(I(i, j)-I_{u v}\right)^{2}} \sum_{i=u-w / 2}^{u+w / 2} \sum_{j=v-h / 2}^{v+h / 2}\left(I^{\prime}(i, j)-I_{u v}^{\prime}\right)^{2}}
$$

where $I_{u v}$ and $I_{u v}^{\prime}$ are averages of each image in a $5 \times 5$ window.

The NCC between two images for pixel $(\mathrm{u} ; \mathrm{v})$ is computed using the $5 \times 5$ subimages as in Eq. (3.2). Note that NCC has a range of $[-1.0,1.0]$. A cost (degree of mismatch) at pixel $(u, v)$, $\operatorname{cost}(u, v)$, is defined as

$$
\cos t(u, v)=\{1.0-\operatorname{NCC}(u, v)\} / 2.0 \text {. }
$$

The cost value approaches 0.0 for similar pixel points and 1.0 for dissimilar pixels.

\subsection{Dijkstra's algorithm}

Dijkstra's algorithm is a global optimization technique that determines the optimal path on the cost space by taking the local minimum operation at each node. To apply to optimal seam line searching, each pixel in an overlapping area is associated with a node, which has 8 neighboring nodes, with four in diagonal directions.

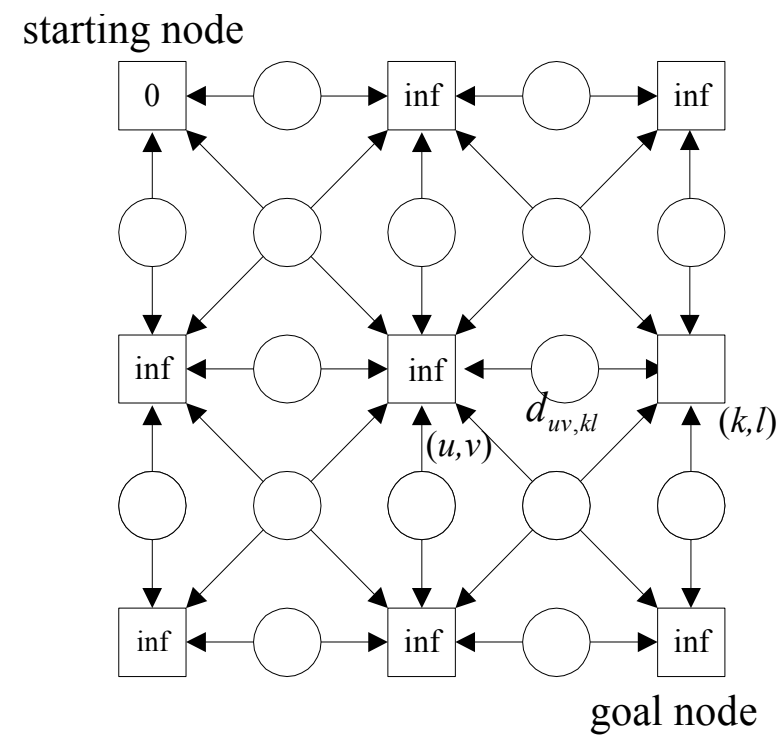

Fig. 3.3. The cost $d_{u v, k l}$ between two nodes $(u, v)$ and $(k, l)$ built by using NCC. 
Let the node at which we are starting be called the initial node. Let $(u, v)$ specify a node and $(k, l)$ be a neighboring node of node $(u, v)$. $\operatorname{NBR}(u, v)$ indicates the set of neighboring nodes of node $(u, v)$. Let the cost $d_{u v, k l}$ be a path cost between two nodes $(u, v)$ and $(k, l)$. Let the global minimum cost $\operatorname{Di}(u, v)$ be a accumulated cost from the starting node to $(u, v)$. Dijkstra's algorithm will assign some initial costs to avoid some areas and will try to improve them step by step.

Assign to every node a tentative cost $D i$ : set it to zero for the starting node and to infinity for all other nodes.

1. Mark all nodes as unvisited. Set the starting node as current. For current node $(u, v)$, consider all its unvisited neighbors and calculate their tentative cost $\mathrm{Di}$.

$$
\operatorname{Di}(u, v)=\min \left\{d_{u v, k l}+D_{i}(k, l) ;(k, l) \in N B R(u, v)\right\}
$$

For example, if current node $(u, v)$ has cost of 6 , and a path cost $d_{u v, k l}$ is 2 , the cost to node $(k, l)$ through node $(u, v)$ will be $6+2=8$. If this cost is less than the previously recorded cost, overwrite the cost. All unvisited neighbors are added to an unvisited set.

2. When we are done considering all neighbors of the current node, mark it as visited. A visited node will not be checked ever again; its cost recorded now is final and minimal.

3. The next current node will be the node with the lowest cost in the unvisited set.

4. If all nodes have been visited, finish and then find the path with the minimum cost between the starting and goal nodes.

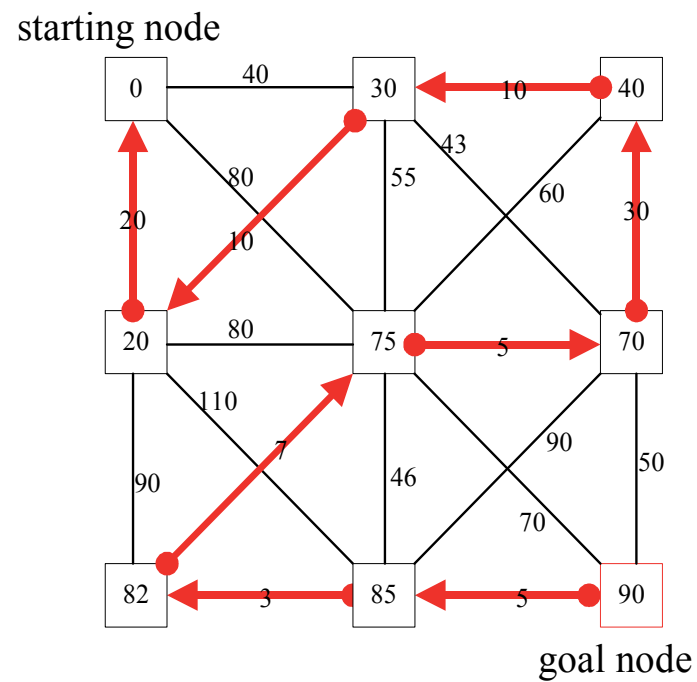

Fig. 3.4. Finding the path with the minimum cost from the goal to staring nodes.

Fig. 3.5 shows four seam-lines detected on four overlapping images using Dijkstra's algorithm on the cost spaces. Each overlapping image is generated from fish-eye images as shown in Fig. 1(c) using a sphere projection method (Coorg and Teller, 2000; Genner, 2006; Kim et al., 2004; Szeliski and Shum, 1997; Yakimovsky and Cunningham, 1978.) The size of each overlapping image is about $30 \%$ of a panoramic image. 


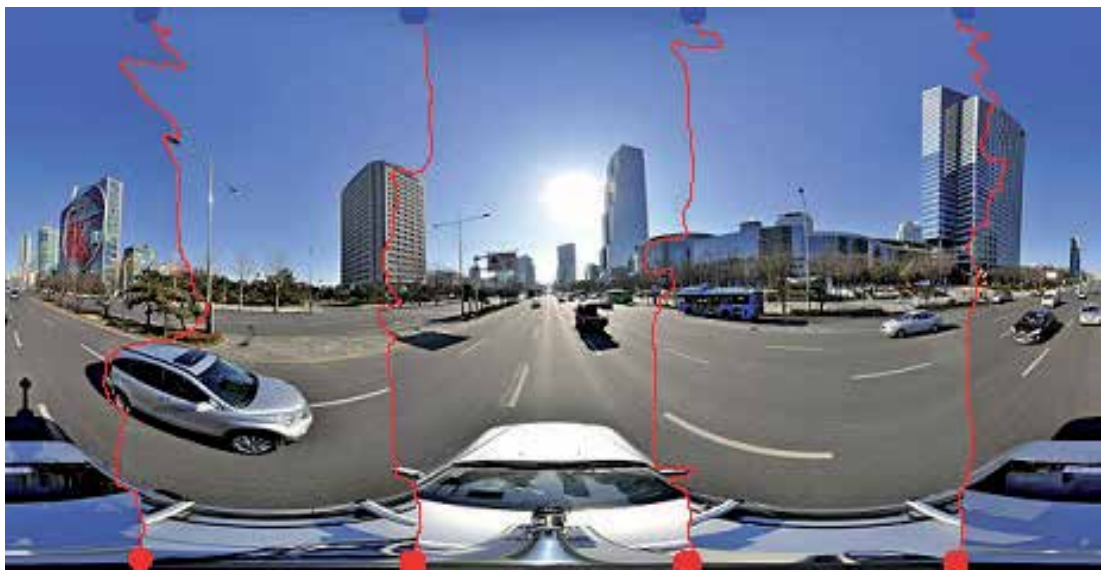

Fig. 3.5. Four seam-lines detected on four overlapping images in a panorama; Blue and red points are starting and goal nodes, respectively.

\section{Fast color blending on seam-lines}

Even though color transitions among all overlapping images are smoothed, the color matching only provides an approximate match. Because color differences among the images corrected by using the color matching method are not enough, Xiong and Pulli (2010) proposed an effective blending method with fast processing speed and high blending quality.

The method calculates the color differences between each $p$ and all points $D(p)$ on each of a seam lines $m_{c}$ and then interpolate $D(p)$ at a pixel $q$ of the blending image (see Fig. 4.1(a)). Finally, the color value $C(q)$ at pixel $q$ are changed as $C(q)=C(q)+D(p)$.

$$
D(q)=\sum_{i=1}^{n} w_{i}(q) D\left(p_{i}\right)
$$

Where $i$ is an index of the seam points consisting of a seam-line $m_{c}$ and weights $w_{i}(q)=\frac{1 /\left\|p_{i}-q\right\|}{\sum_{j=1}^{n} 1 /\left\|p_{j}-q\right\|}$ are the inverse coordinate distance to the boundary pixels.

Additionally, they proposed the creation of a color difference distribution process to enforce color consistency for 360-degree panorama. They attenuate the color of the pixel $q$ in the blending area on the current scan line with

$$
C(q)=C(q)+\left(1-\frac{x}{x_{b}}\right) D(q)
$$

where $x$ and $x_{b}$ are the horizontal distances between $q$ and the seam point $p$ of the seam line $m_{s}$ and between $p$ and the end of the blending area, respectively, in Fig. 4.1(b). 


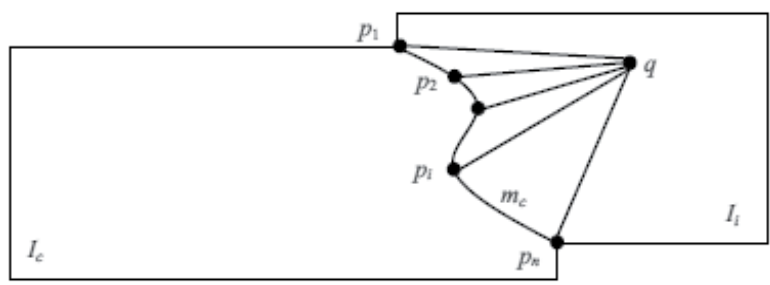

a)

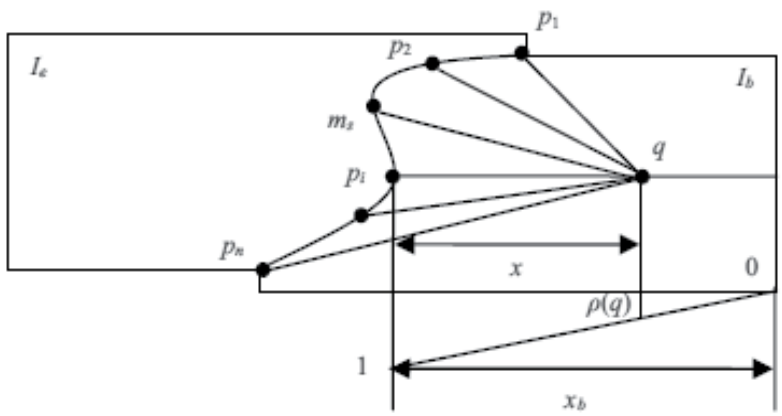

b)

Fig. 4.1. Image blending on a seam line and color difference distribution for 360-degree panoramic images (Xing and Pulli, 2010).

\subsection{The proposed method}

If overlapping images are well aligned, Xiong and Pulli (2010) proposed method works perfectly. However, poor image alignment due to parallax, registration errors, and radial distortions can lead to poor blending results in general.

To cope with this problem, we propose filtered color differences using multiple major color differences detected on a seam-line. When the seam points are well aligned, the difference between the neighboring points will be smoothly changed. Because seam points with misalignment cause sudden changes, we simply filter out these seam points and keep good aligned seam points. And we then replace the misaligned seam points with interpolated color differences using the ends of two neighbor seam points on a seam-line. We detect smoothly changed color differences using a median filter and the standard deviation of the changes.

\subsection{Detection of good aligned seam points}

Fig. 4.2(a) shows an overlapping image that is located at the rightmost side of the panoramic image shown in Fig. 3.5. Fig. 4.2(b) shows a graph of color differences corresponding to the seam points of the seam line shown in Fig 4.2(a). If the seam points are well aligned, the differences will be smooth like the differences for seam points in the sky area.

Before applying Eq (4.2), we have to remove the color differences of poorly aligned seam points. To detect smooth changing parts, we use a threshold based on the standard deviation of the color differences. The graph in Fig 4.2(c) depicts the standard deviations of the color differences for each channel. The standard deviation of poorly aligned seam points is higher than that of the sky and road. 
To evaluate whether seam points are poorly aligned or well aligned, we first build a histogram that accumulates the standard deviation in the horizontal direction, shown in Fig 4.2(c) as the thick green curve, and then find the peak point ${ }^{\prime * \prime}$ in the thick green. A dotted red horizontal line in Fig. 4.2(c) chosen by the peak point is a reference standard deviation. We add a margin into the reference standard deviation to calculate a threshold, a red horizontal line in Fig. 4.2(c).

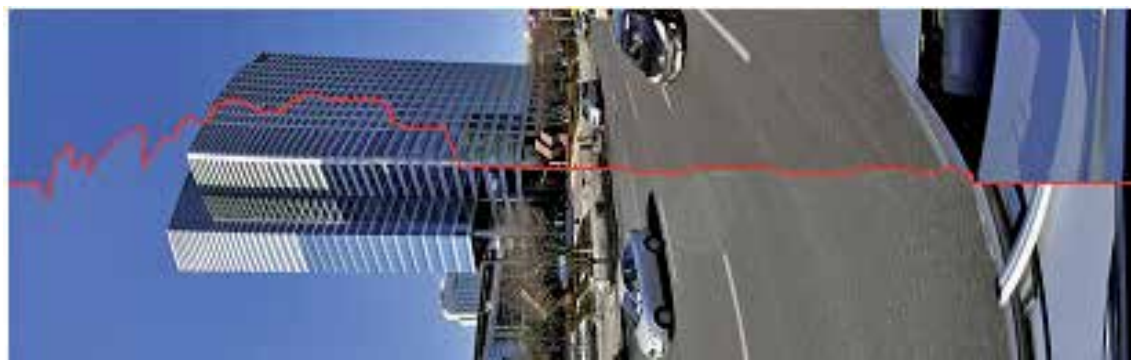

a) A seam-line
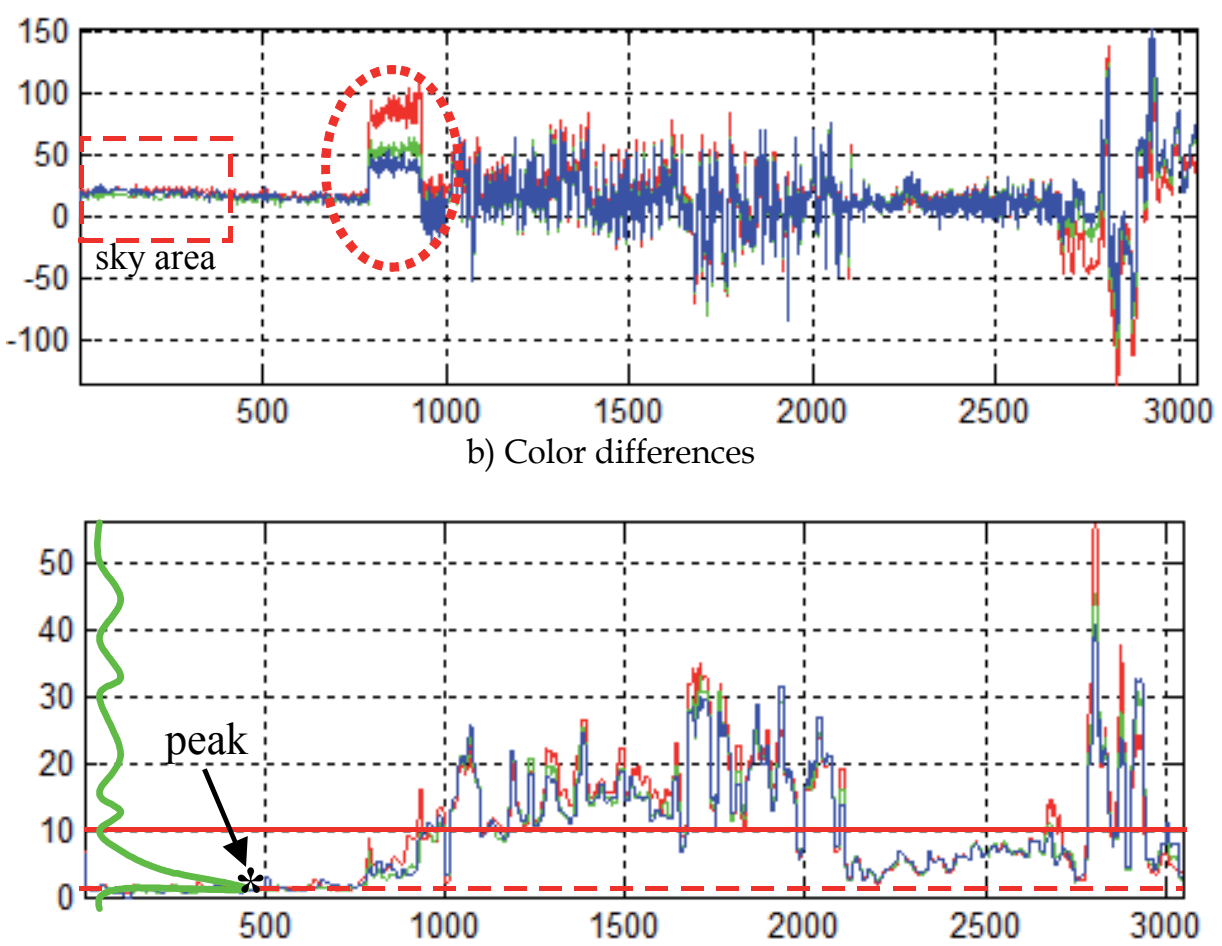

c) Standard deviation and histogram

Fig. 4.2. The color differences and standard deviation of seam points; ${ }^{*}{ }^{\prime}$ is the most peak point in a histogram and the dotted and solid horizontal lines are the reference standard deviation and threshold, respectively, in c).

We cannot remove the color differences in a dotted circle in Fig. 4.2(b) using only this single threshold, because the standard deviation of the color differences of seam points in the circle 
is low like the reference standard deviation. To remove the color differences of those seam points on the seam-line, we use the same process to build the second histogram using the color differences of the remaining seam points, which are determined by using the first histogram built by using the standard deviation.

After detecting a peak point on the second histogram as a reference of color difference, two thresholds are calculated by adding and subtracting a margin. In our experiments, the margin is set as 15 . If a single reference is only applied, bad blending will occur as shown in Fig 4.3.

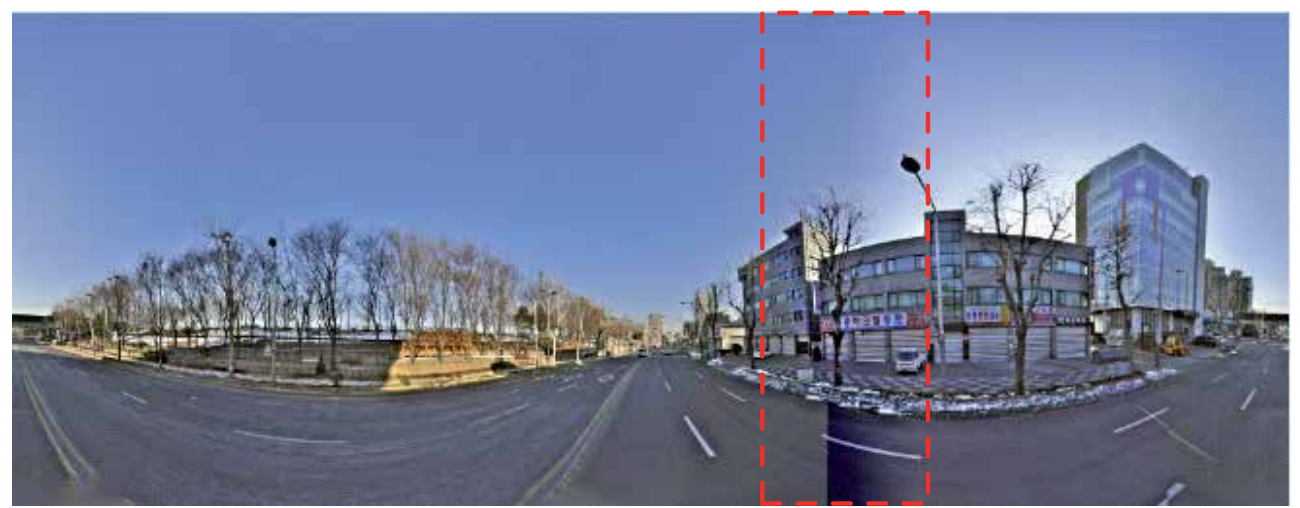

Fig. 4.3. Our extended algorithm with a single peak point

To suppress this problem, we choose multiple peak points in the histogram. If a peak point is including over $20 \%$ of the number of all seam points on the seam-line, the peak point becomes one of the references of color difference. Figs. 4.4(a) and 4.4(b) show color blending results by using one and two peak points chosen by our algorithm with each second histogram, respectively.

As the final step, we have to replace the color differences of seam points detected as poorly aligned seam points during the previous step. A line or curve is built by using both two ends of neighbor seam points from well-aligned seam points.

Figs. 4.5(a) and 4.5(b) show panoramic images created by using method of Xiong and Pulli (2010) and our extended method, respectively. When comparing the area in the dotted circle in Fig. 4.5(a), our extended method made a better result then that by Xiong and Pulli's method. However, our extended method did not perform as well within the dotted square in Fig. 4.5(b). This is originated from big differences among the values of the red, green, and blue channels of one or two end points of the remained seam points as reference color differences. If we change color differences into gray differences, this phenomenon will be disappear. However, subtle color differences among RGB channels could not recovered. We apply a median filter with a couple of seam points around the two end seam points to remove the incorrect differences. Fig. 4.6(a) shows an image including the phenomenon that is an overlapping image in the dotted square in Fig. 4.5(b) and a graph of color differences. Figs. 4.6(b) and 4.6(c) show a graph of filtered color differences and a final result using the filtered color differences, respectively. 


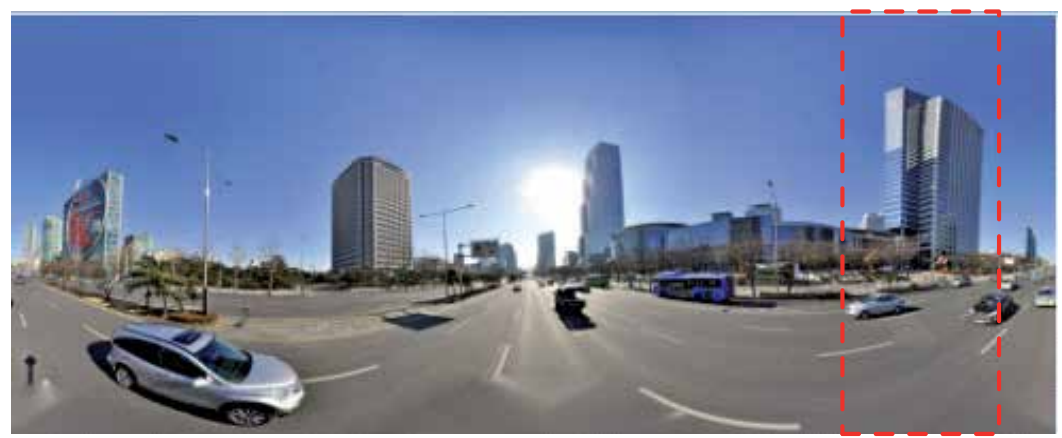

a) A single peak point

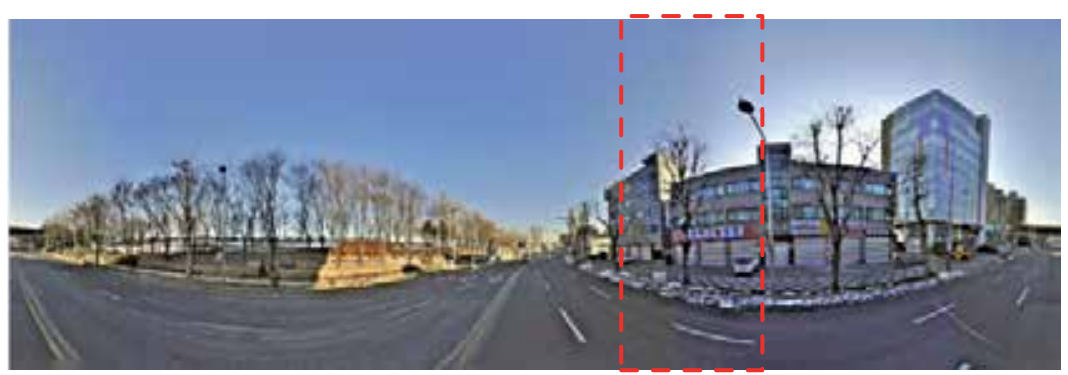

b) Two peak points

Fig. 4.4. A panorama re-created by our extended seam-line blending algorithm with multiple peak points chosen in a histogram of color differences

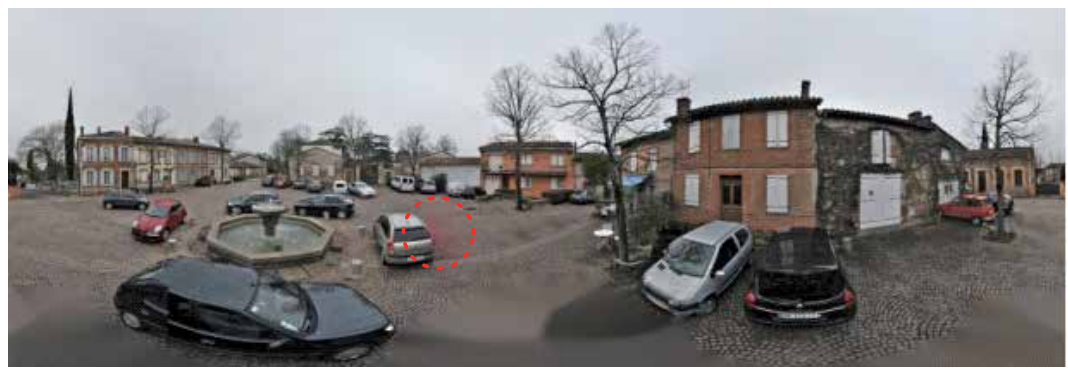

a)

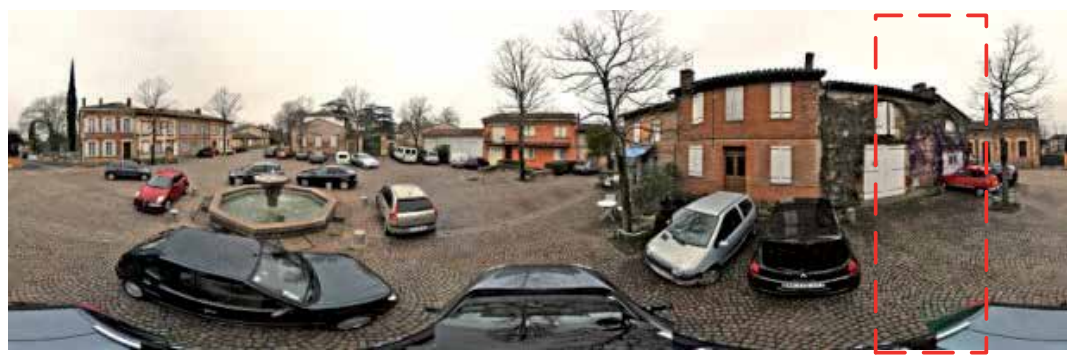

b)

Fig. 4.5. Comparison between a) Xiong and Pulli (2010) method and b) The extended method. 

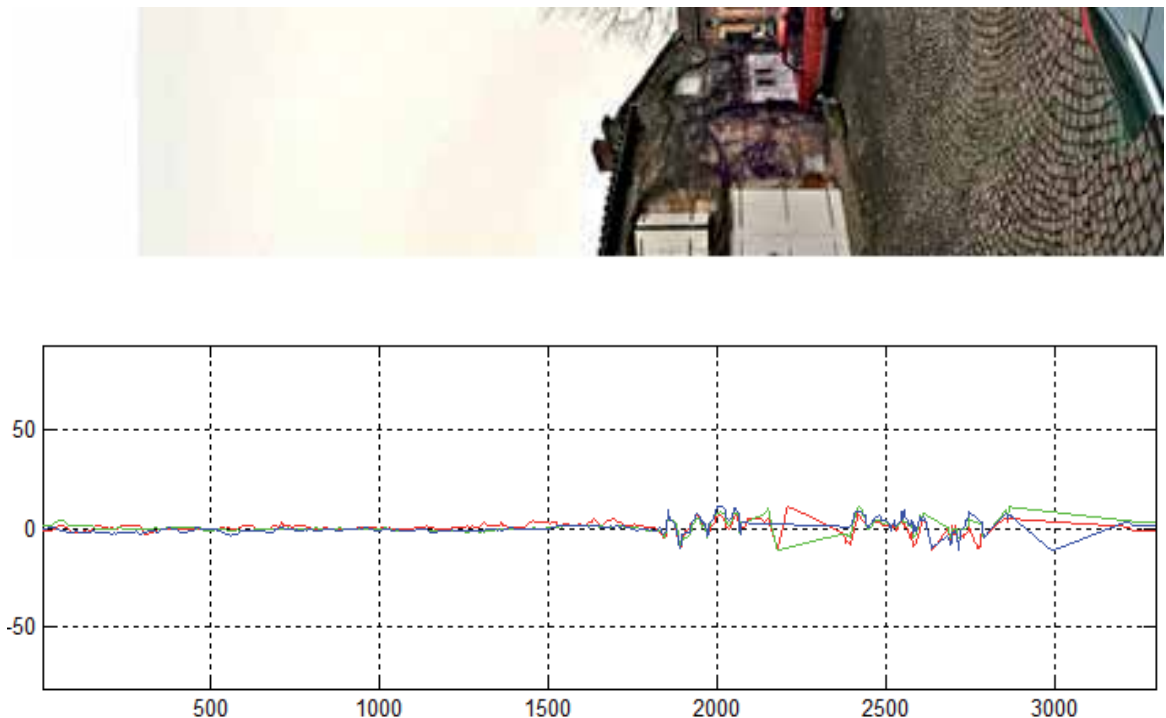

a) Linear interpolated color differences

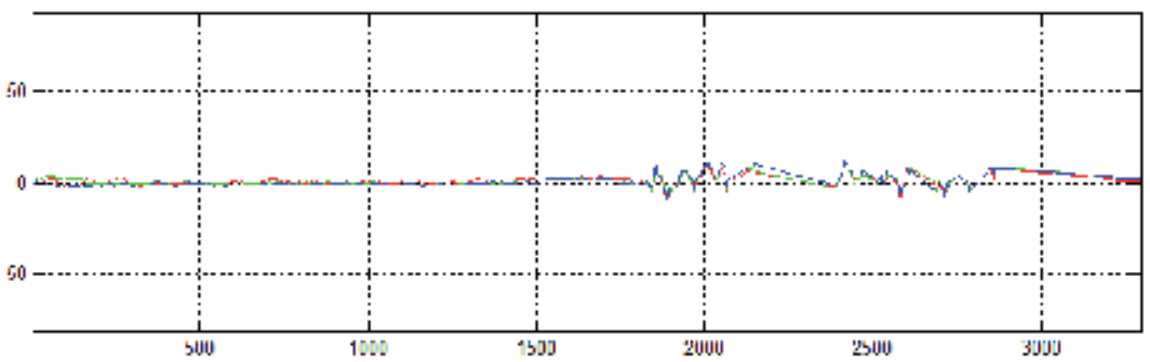

b) Color differences by median filter

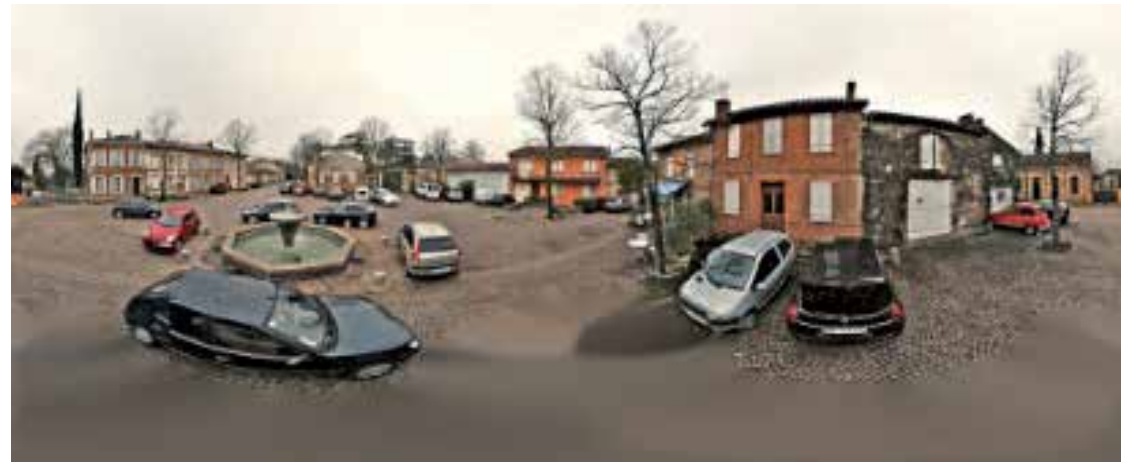

c) Final panoramic image

Fig. 4.6. Median filter to remove incorrect interpolated color differences and a final panoramic image. 


\section{Color balance and saturation}

To make the final panorama more aesthetically pleasing, we apply additional processing to increase contrast, color balance, and color saturation. The additional processing also has the added benefit of making the collection process more tolerant to different lighting conditions.

We achieve color/white balance by using existing auto-white balance algorithm (employed in many image-editing applications, such as Adobe Photoshop). This algorithm computes histograms for each color channel (red, green, and blue) and then in each channel "discards" the $0.5 \%$ darkest and $0.5 \%$ lightest pixel colors from the histogram and then stretches the resulting histogram to [0, 255] effectively making all pixels that fell in the discarded ranges "black" and "white" respectively.

The above process has two effects. First, it increases the apparent contrast of the image. Second, it can cause a noticeable hue shift because the algorithm operates on the color channels individually. This is desirable for panoramas taken outdoors in natural light because it makes overly "cool" scenes appear warmer and vice versa--providing a good overall aesthetic improvement robustly under varying weather conditions. On the other hand, when the algorithm processes scenes with significantly dark scenes (such as panoramas in tunnels or those taken at night under artificial lighting) the hue shift will be much more pronounced and often produces very poor results. Thus, this color balance algorithm is only applied to images that is taken under decent lightly conditions. Figure 5 illustrates the effect of this algorithm.
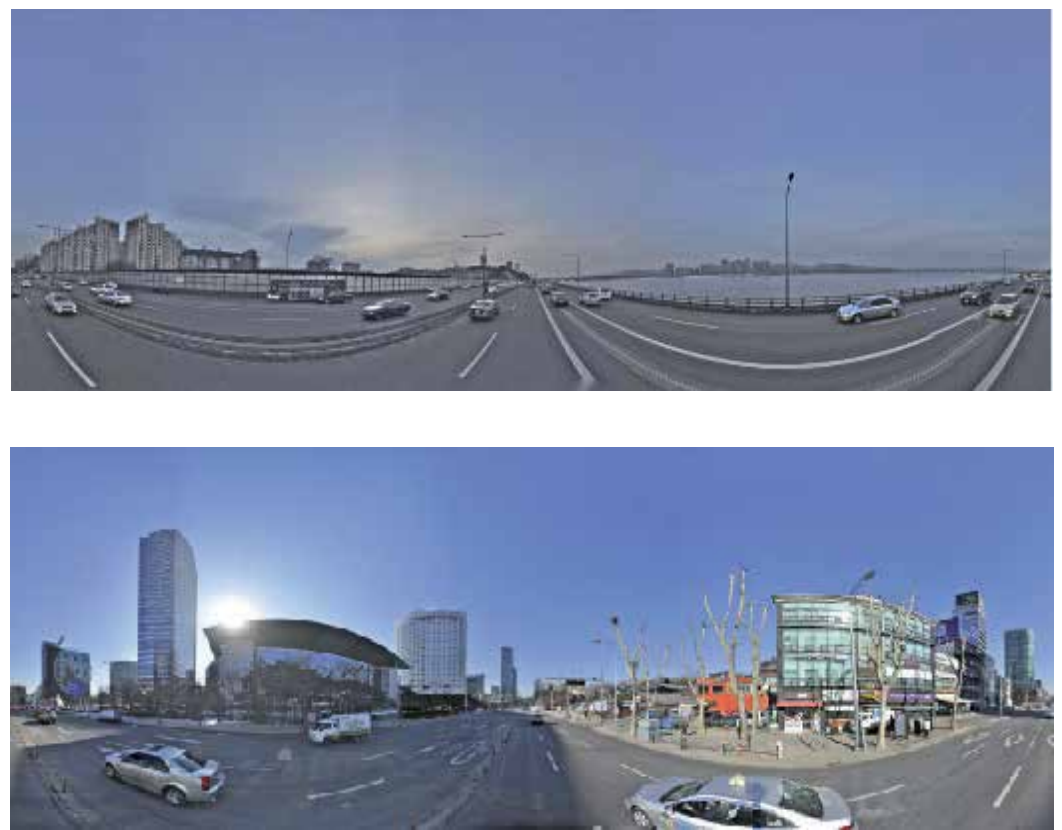

a) Only our extended method 

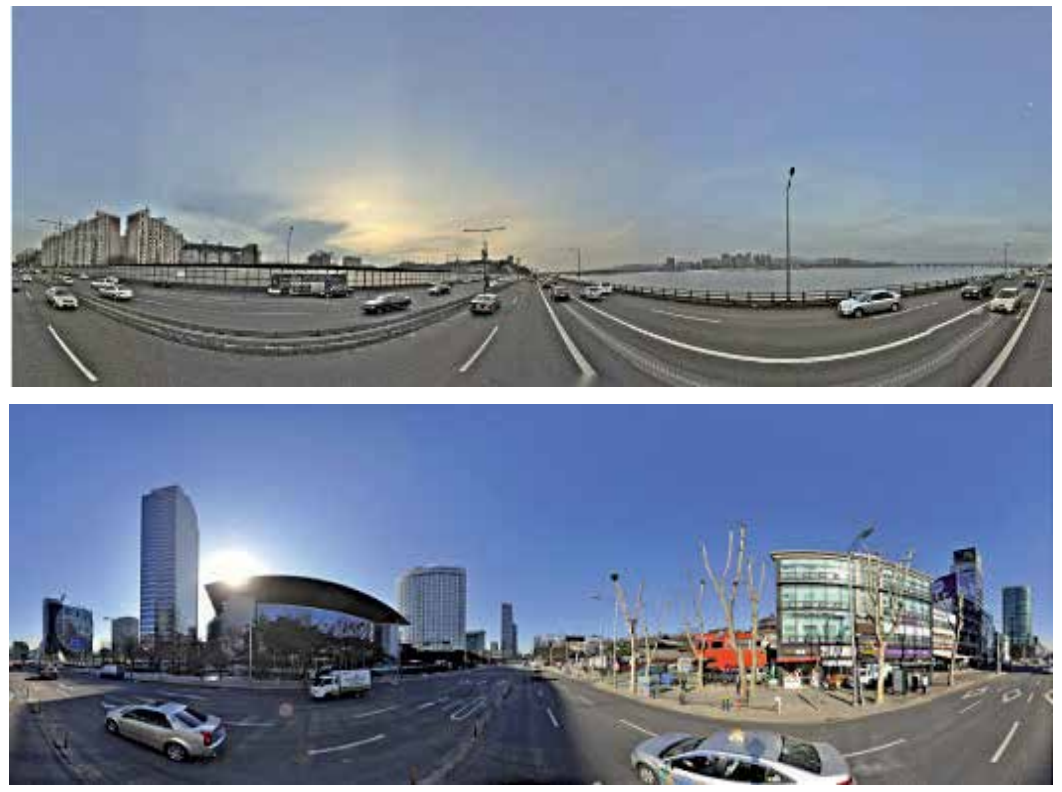

b) With more Color balance and saturation

Fig. 5. Example panoramas before a) and after b) color balance and saturation adjustment.

\section{Conclusion}

In this chapter we introduced a novel panorama-stitching algorithm. We described each stage of the algorithmic pipeline in detail, namely a channel-correction algorithm that normalizes luminance and chrominance of the projected images prior stitching; a seam-line finding algorithm that finds the optimal transition between two overlapping images; a color blending algorithm that blends the seam-lines; and finally, a color enhancement algorithm that adjusts the overall contrast, color, and saturation of the panoramic image.

In the algorithm, we particularly address the shortcomings of previous approaches, where poor results are often observed from the color-blending algorithm due to poorly aligned points from the individual images on the seam-lines. Our proposed algorithm removes outliers on the seam-lines due to misalignment by using histograms, color differences, and standard deviation of the color differences. The outliers are replaced by linearly interpolated points using the color differences of neighbor well-aligned seam points.

We have tested the proposed algorithm on millions of outdoor panoramas and it has been proven that the algorithm is robust under most lighting and weather conditions. The algorithm described in this paper is not only bounded to Earthmine's collection system but any system that generates panorama images.

\section{References}

Bellman, R., (1957). Dynamic Programming. Princeton Univ. Press.

Brown, M. and Lowe, D., (2007). Automatic Panoramic Image Stitching using Invariant Features. International Journal of Computer Vision, 74(1):59-73. 
Chen, S. E., (1995). QuickTime VR-an image-based approach to virtual environment naviation. In ACM SIGGRAPH 1995 Conference Proceedings, pp. 29-38, Los Angeles.

Chon, J., Fuse, T., Shimizu, E., Shibasaki, R., (2007). Three-dimensional image mo saicking using multiple projection planes for 3-D visualization of roadside standing buildings. IEEE Transactions on Systems, Man and Cybernetics, Part B (Cybernetics) 37 (4):771-783.

Chon, J., Kim, H., and Lin, C.S., (2010). Seam-line determination for image mosaicking: A technique minimizing the maximum local mismatch and the global cos., ISPRS Journal of Photogrammetry and Remote Sensing, 65(1): 86-92.

Chou, J.S., Qian, J., Wu, Z., Schramm, H., (1997). Automatic mosaic and display from a sequence of peripheral angiographic images. Proceedings SPIE Medical Imaging, 3034:1077-1087.

Coorg, S. and Teller, S., (2000). Sphereical Mosaics with Quaternions and Dense Correlation. International Journal of Computer Vision, 37(3):259-273.

Dijkstra, E.W., (1959). A note on two problems in connexion with graphs. Numerische Mathematik, 1: 269-271.

Efros, A., Freeman, W., (2001). Image quilting for texture synthesis and transfer. In: Proceedings International Conference on Computer Graphics and Interactive Techniques. pp. 341-346.

Genner, D.B., (2006). Generalized camera calibration including Fish-eye lenses, Int. J. Computer Vision, 68(3): 239-266.

Goldman, D. B. (2011). Vignette and exposure calibration and compensation. IEEE Transactions on Pattern Analysis and Machine Intelligence, 32(12):2276-2288.

Hartley, R. and Zisserman, A., (2003). Multiple View Geometry. 2nd Edition, Cambridge University Press.

Irani, M. and Anandan, P., (1998). Video Indexing based on mosaic representations. Proceedings of the IEEE, 86(5): 905-921.

Irani, M., Hsu, S., and Anandan, P. (1995). Video compression using mosaic representations. Signal Processing: Image Communication, 7:529-552.

Kass, M., Witkin ,A., and Terzopoulos, D., 1987. Snakes: Active contour models. International Journal of Computer Vision. 1(4): 321-331.

Kerschner, M., (2001). Seam-line detection in colour orthoimage mosaicking by use of twin snakes. ISPRS Journal of Photogrammetry and Remote Sensing, 56 (1):53-64.

Kim, S. J. and Pollefeys, M., (2008). Robust Radiometric Calibration and Vignetting Correction. IEEE Transactions on Pattern Analysis and Machine Intelligence, 30(4):562-576.

Kim, W.S., Steinke, R.C., Steele, R.D., Ansar, A.I., (2004). Camera Calibration and Stereo Vision Technology Validation Report. JPL D-27015.

Kumar, R., Anandan, P., Irani, M.,Bergen, J., and Hanna, K. (1995). Representation of scenes from collections of images. In IEEE Workshop on Representaions of Visual Scenes, pp. 10-17, Cambridge, Massachusetts.

Lee, M.-C., ge Chen, W., lung Bruce Lin, C., Gu, C., Markoc, T., Zabinsky, S. I., and Szeliski, R. (1997). A layered video object coding system using sprite and affine motion model. IEEE Transactions on Circuits and Video Technology, 7(1):130-145.

Lewis, J. P., (1995). Fast Normalized Cross-Correlation. Vision Interface, pp. 120-123. 
Leymarie, F. and Levine, M.D., 1993. Tracking deformable objects in the plane using an active contour model. IEEE Transactions on Pattern Analysis and Machine Intelligence. 15(6): 617-634.

Lowe, D.G., (2004). Distinctive Image Features from Scale-Invariant Keypoints. International Journal of Computer Vision, 60(2):91-110.

McGlone, C., E. Mikhail and J. Bethel, (2004). Manual of Photogrammetry, 5th Edition, ASPRS.

Milgram, D.L., (1975). Computer methods for creating photomosaics. IEEE Transactions on Computers, C-24(11):1113-1119.

Milgram, D.L., (1977). Adaptive techniques for photomosaicking. IEEE Transactions on Computers, C-26(11):1175-1180.

Poynton, C. ,(2002). Digital Video and HD: Algorithms and Interfaces. Morgan Kaufmann; $1^{\text {st }}$ edition (December 2002)

Tepdosio, L. and Bender, W., (1993). Salient video stills: Content and context preserved. In ACM Multimedia 93, pp. 39-46, Anaheim, California

Szeliski, R., (1996). Video mosaics for virtual environments. Computer Graphics Applications 16 (3): 22-30.

Szeliski, R., (2011). Computer Vision: Algorithms and Applications. Springer.

Szeliski, R. and Shum, H.-Y., (1997). Creating full view panoramic image mosaics and texture-mapped models. In ACM SIGGRAPH 1997 Conference Proceedings, pp. 251-258, Los Angeles.

Tiilikainen, N.P., (2007). A Comparative Study of Active Contour Snakes. Copenhagen University, Denmark.

Williams, D.J. and Shah, M., (1990). A fast algorithm for active contours. CVGIP: Image Understanding. 55(1): 14-26.

Xiong, Y. and Pulli, K., (2010). Color Matching for High-Quality Panoramic Images on Mobile Phones. IEEE Transactions on Consumer Electronics, 56(4), 2592-2600.

Yakimovsky, Y. and Cunningham, R.T., (1978). a system for extracting three-dimensional measurements from a stereo pair of TV cameras, Computer Graphics and Image Processing, 7, 195-210.

Zomet, A., Levin, A., Peleg, S., and Weiss, Y., (2006). Seamless Image Stitching by Minimizing False Edges. IEEE Transactions on Pattern Analysis and Machine Intelligence. 15(4): 969-977. 


\title{
Assessment of Stereoscopic Precision - Film to Digital Photogrammetric Cameras
}

\author{
Benjamín Arias-Pérez¹, Diego González-Aguilera1, \\ Javier Gómez-Lahoz ${ }^{1}$ and David Hernández-López ${ }^{2}$ \\ ${ }^{1}$ High Polytechnic School of Avila, Department of Cartographic and Land Engineering, \\ University of Salamanca \\ ${ }^{2}$ Regional Development Institute, Albacete, University of Castilla-La Mancha
} Spain

\section{Introduction}

In the generation of the three more important photogrammetric products, digital terrain models (DTM), orthophotos and maps derived from compilation, issues such as direct georeferencing, managing a high volume of data and automatic measurements (matching), are really important. However, within the photogrammetric workflow still exists tasks that remain manual or that require user interaction. The generation of cartography through restitution is one of the tasks that require an intense user interaction despite the great advances in the sector. On the other hand, the constant emergence of new large and medium format digital sensors and their incorporation into large photogrammetric projects has prompted different stakeholders demand and thus a greater need to the knowledge of the precision, correctness and reliability of these sensors, especially when most existing photogrammetric software do not allow a detailed analysis of the results. That is why nowadays is still relevant to consider the photogrammetric precision reached by an operator measuring on a digital image and compare it with that achieved by measuring on a scanned film image, considering always that both dataset are provided with similar input conditions (pixel size, measurement device, expertise of the operator, etc.)

This chapter aims to address this issue in detail through a study of manual stereoscopic precision measured on original digital image and digitalized film images. After this introduction, Section 2 will address a comprehensive bibliographic review of major studies in this line, from those made in the field of analogical photogrammetry to the modern largeformat digital cameras. Section 3 will describe in detail the main materials and methods used in this study. Section 4 will focus on showing experimental results obtained in three different study cases with a discussion of them. The last section we will highlight the main concluding remarks from this study.

\section{State of the art}

Photogrammetric Community has always tested new tools and methods with the aim of guaranteeing that the results achieved are equal to or better than traditional ones. In this 
sense, one of the first tests performed was to check the precision of the film cameras that finally replaced the plate cameras (Grifoni, 1949). From these studies important conclusions were derived confirming the superiority of the plate cameras in terms of precision. However, for small and medium scales, film cameras were fully reliable. Later, Lehmann (1955) in the framework of the Organisation Europeenne d'Etudes Photgrammetriques Experimental OEEPE investigates the precision of restitution based on several factors such as the field of view, photographic material (film or plate), the method of measuring, the user, the type of instrument (plotter, etc.). To develop this work, Switzerland offered a test field located in the Rhine valley near Oberriet, which covers an area of 1,5 x1,5 km with 600 control marks with planimetric and altimetric coordinates. Furthermore, in an area of $4 \times 4$ $\mathrm{km}$ points were spaced every $500 \mathrm{~m}$. This trend was followed by a total of 7 schools in different countries ( 1 in Switzerland, Austria, Italy and the Netherlands and three in Germany), using different restitution instruments. Some of the centers that performed the measurements published their reports including aspects such as: times of measurement, methods of operation, problems encountered and their solutions, and even the measurements made by the operators (Gotthardt, 1955; Brucklacher, 1955, and Förstner, 1955, Commission C., Ablauf der Messung OEEPE Deroulment Zeitlicher chronologique des observations, 1955), while the results were discussed in later publications (Gotthardt, 1958, and Stickler, 1959; Stoch, 1961). At the same time as the OEEPE began its work, the International Society of Photogrammetry, ISP, showed their concerns about the restitution of cadastral maps (Härry, 1954), land consolidation (Härry, 1955), establishing plans for urban areas (Dubuisson, 1955) and small scale mapping (Blachut, 1955). In 1975, the analysis of planimetric and altimetric precision on the restitution was revived again but this time through the angular field factor. Stark (1975) used a total of 4 cameras with varying focal lengths and taking images at different flight altitudes. A total of 23 sets of points distributed regularly around the stereoscopic model were measured and analyzed for each stereoscopic model. The study followed that the altimetric mean error decreases continuously as the image angle increases, while the planimetric mean error is practically independent of this angle. On the other hand, another aspect that has provided a particular interest from the International Photogrammetric Community is the comparison of stereoscopic and monoscopic measurements (O'Connor, 1967; Karara, 1967; Trinder, 1986). To this end, manual stereoscopic measurements involving human operators were also developed in some tests to determine the stereoscopic accuracy achieved by restitution operators (Zorn, 1965, Krakau, 1970).

With the advent of large format digital cameras in 2000, studies comparing the analogdigital technology have become inevitable. Dörstel (2003) analyzed the precision of largeformat digital camera DMC using four flights at different heights while preserving the ratio of base/height $(b / h)$. Dörstel performed 10 measurements of each point and use different types of points that allow him to contrast the empirical and theoretical precision. Alamús et al. (2005) contrasted the ground coordinates (measured with GPS) with those obtained by stereoscopic measurement, and making these measurements with film camera, RC30 $(b / h=0,6)$, and digital, DMC $(b / h=0,3)$. Use 11 points in a flight with a GSD of $0,08 \mathrm{~m}$ (Amposta block), and 21 points on another flight of $0,5 \mathrm{~m}$ (Caro block). It provides data on how many times are measured points, or if they are homogeneously distributed in the model, or classified in some way, how many operators are involved in the measurements, 
etc. The results show that the smallest ratio $b / h$ for DMC camera is compensated with higher precision in stereo measurements, reaching comparable values in all components $(X, Y, H)$, and for the two flights. Subsequently, other specific tests were performed to determine the altimetric stereoscopic precision. Points were measured in several contiguous stereo models near to the so-called Von Gruber points. The results showed that the precision in $\mathrm{Z}$ is worse in the case of digital camera which can be due to the topography, or because that overlap areas are different in digital and film images.

More recently, Arias and Gomez-Lahoz (2009) conducted an empirical study of stereoscopic precision. Finally, Spreckels et al. (2010) reported the results obtained in the project DGPF "Evaluation of Digital Photogrammetric Camera Systems", within the working group "stereoplotting". Multiple cameras were used in this project: Film Camera Zeiss RMK Top 15; large format digital cameras UltraCamX Vexcel Imaging and Intergraph / ZI DMC, and the combination of four medium format cameras Digi-CAM Quattro IGI. The main outline of the project show a precision better than 0,9 pixel in $X Y$ and 1,4 pixel in $Z$.

\section{Materials and methods}

\subsection{Photogrammetric sensors: Digital}

\subsubsection{DMC}

This digital sensor is based on a multi-cone matrix, so that four sensors can provide a large format CCD (7.000 x 4.000-pixel, 12 micron pixel size), which capture the image at the same time (synchronous operation) (Fig. 1). Panchromatic cones are slightly inclined, so they have a small common area, which is then used to generate the so-called virtual image size of $13.824 \times 7.680$ pixels (height $x$ width). The color information is obtained from four CCDs with a smaller size, but that capture the entire scene. A whole high-resolution color image can be obtained automatically using pan-sharpening method.
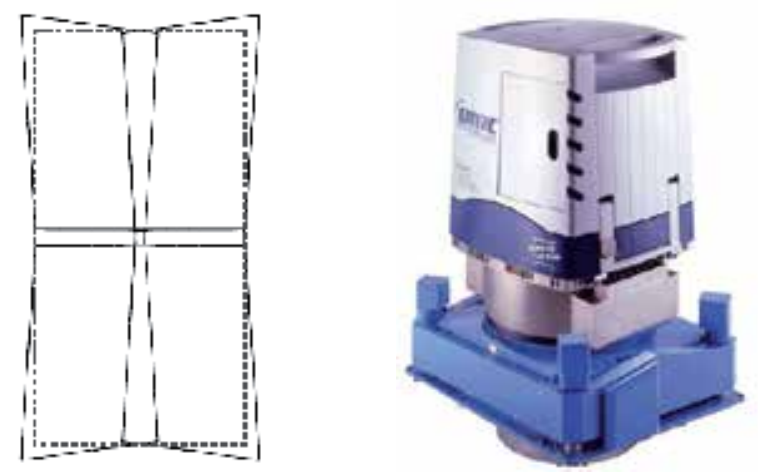

Fig. 1. Left: images of the four cones (solid line), and the virtual image (dashed line). Right: DMC digital camera.

\subsubsection{UltraCam}

The UltraCamD camera design is based on the use of 9 sensors CCDs (each of $4.000 \times 2.700$ pixels) with pixel size of 9 microns (Fig. 2). Each cone has the same field, but the CCDs are 
arranged in various positions within the focal plane. The idea is that not all cones are exposed to the same time, but at the same place (operation syntopic). A cone acts as a master cone, and that defines the image coordinate system. The other images are paired as secondary parts in this main frame defined by the master cone. The final image has a single central perspective and has a size of $11.500 \times 7.500$ pixels.
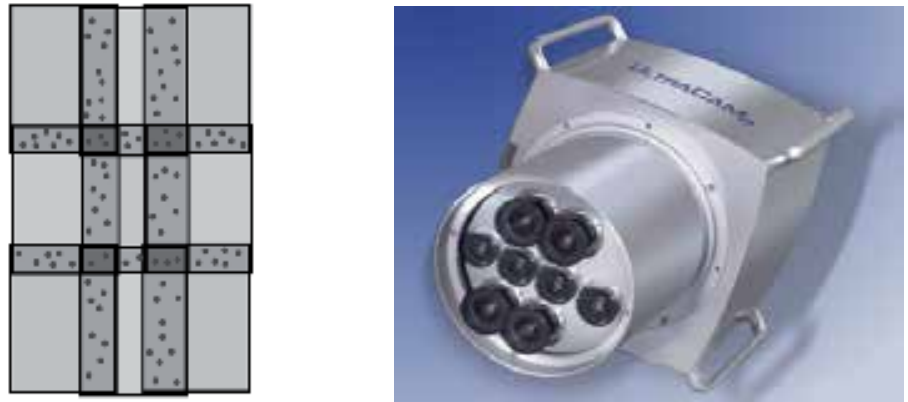

Fig. 2. Left: 9 CCDs that form the complete image of the camera UltraCam.

Right: camera UltraCamD.

\subsection{Photogrammetric sensors: Film}

In this case the camera used was the Leica RC30 camera, widely used in aerial photogrammetry industry. It allows two settings: 15/4 UAG-S with a focal length of $15 \mathrm{~cm}$ (the one used in the measurements), and 30/4 NAT-S with a focal length of $30 \mathrm{~cm}$. In both cases, the format corresponds to a film width of $240 \mathrm{~mm}$. But due to the intrinsic characteristics of these cameras (fiducial marks, marginal information) the effective width is smaller.
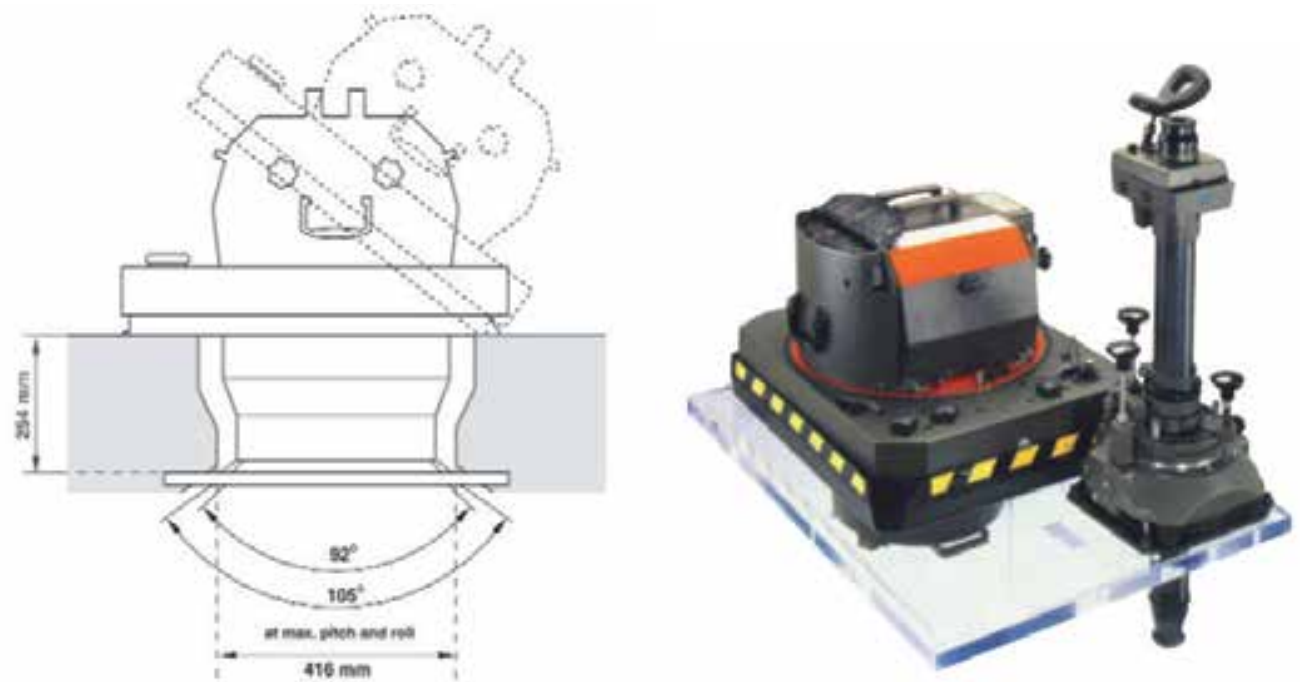

Fig. 3. Left: diagram of Leica RC30 with 15/4 UGS (in PAV30 mount). Right: Leica RC 30 camera (in PAV30 mount) and the NSF3-E Navigation Sight.

The scanner used to convert the film to digital format was Vexcel UltraScan 5000. 


\subsection{Methods}

The following precision analysis have been established in this study: XY precision, $Z$ precision, relationship between planimetric and altimetric precision, comparison of means, analysis of agreement, personal equation and relative relief. Measurements were taken with the analytical plotter Leica SD2000 and the photogrammetric digital workstation Digi3D.

\subsubsection{Theoretical precision in planimetry}

The theoretical XY precision is directly proportional to the scale of the image, $m_{b}$, and the measurement precision of the image, $\sigma_{i}$ :

$$
\sigma_{x y}=\sigma_{i} * m_{b}
$$

The precision of the measure on the image plane, $\sigma_{i}$ usually $\pm 6 \mu \mathrm{m}$ (Kraus, 1993) can be expressed in terms of pixel size, $p x$, as a fraction $(1 / k)$. This value $k$ can be considered as an indicator of measurement precision in the image.

$$
\sigma_{i}=\frac{p x}{k} \Rightarrow \sigma_{x y}=\frac{p x}{k} * m_{b}
$$

Moreover, the product of pixel size for image scale provides the pixel size in the ground, GSD (Ground Sample Distance):

$$
G S D=p x * m_{b} \Rightarrow \sigma_{x y}=\frac{G S D}{k}
$$

Thus, the precision observed in $X Y$ can be expressed as a fraction of the GSD. Once the empirical planimetric standard deviation, $S_{X Y}$, is obtained, the empirical measurement precision of the image, $S_{i}$ is get. From $S_{i}$ the value of $k$ can be computed which is a good value of comparison between cameras.

$$
\begin{aligned}
& S_{x y}=S_{i} * m_{b} \Rightarrow S_{i}=\frac{S_{x y}}{m_{b}} \\
& S_{i}=\frac{p x}{k} \Rightarrow k=\frac{p x}{S_{i}}
\end{aligned}
$$

From this expression it follows that the higher $k$, the better precision.

It is important to note that $\sigma$ expresses the theoretical precision while $S$ expresses the empirical standard deviation which is determined from measurements.

\subsubsection{Theoretical precision in altimetry}

The theoretical precision in $Z, \sigma_{Z}$, depends on the precision of measurement of the horizontal parallax, $\sigma_{P x}$, the image scale, $m_{b}$, and the ratio height/base, $H / B$ (Kraus, 1993; Schiewe, 1995):

$$
\sigma_{z}=\sigma_{P x} * m_{b} * \frac{H}{B}
$$


The measurement precision of the horizontal parallax can be replaced by the measurement precision in the image plane, $\sigma_{i}$. The ratio height/base can be replaced by the ratio focal/base $(c / b)$, then:

$$
\sigma_{z}=\sigma_{i} * m_{b} * \frac{c}{b}
$$

The precision of the measure in the image plane, $\sigma_{i}$, can be expressed in terms of pixel size as a fraction of it. In this case, it is assigned a value of $1 / k$ :

$$
\begin{aligned}
\sigma_{i} & =\frac{p x}{k} \\
\sigma_{z} & =\frac{p x}{k} * m_{b} * \frac{c}{b}
\end{aligned}
$$

Moreover, the product of pixel size for image scale provides the pixel size in the ground, GSD:

$$
\begin{aligned}
& G S D=p x * m_{b} \\
& \sigma_{z}=\frac{G S D}{k} * \frac{c}{b}
\end{aligned}
$$

As can be seen, precision in $Z$ can also be expressed in terms of the GSD, the focal length and photobase. This is a function of longitudinal overlap, $R_{L}$, together with the image width:

$$
b=\left(1-R_{L}\right) * \text { width }
$$

The value $c / b$ affects proportionally the $Z$ precision, so that the higher the value of this ratio less precision in $Z$.

\begin{tabular}{|c|c|c|c|c|}
\hline Camera & $c(\mathrm{~mm})$ & Width $(\mathrm{mm})$ & $\begin{array}{c}b\left(R_{\mathrm{L}}=60 \%\right) \\
(\mathrm{mm})\end{array}$ & $c / b$ \\
\hline Film & 150 & 220 & 88 & 1,704 \\
\hline DMC & 120 & 95 & 38 & 3,158 \\
\hline UltraCamD & 100 & 67,5 & 27 & 3,704 \\
\hline UltraCamX & 100 & 68,4 & 27,36 & 3,655 \\
\hline
\end{tabular}

Table 1 . Ratios $c / b$ or various photogrammetric aerial cameras, calculated for a longitudinal overlap of $60 \%$.

A comparison of details leads to the study of the ratio of precisions with two different cameras (D: Digital, UltraCamD or DMC; A: Analog-Film):

$$
\frac{\left(\sigma_{z}\right)_{D}}{\left(\sigma_{z}\right)_{A}}
$$

The comparison must be made by measurements from similar flights, which have the same GSD: 


$$
\frac{\left(\sigma_{z}\right)_{D}}{\left(\sigma_{z}\right)_{A}}=\frac{\left(\frac{G S D}{k} * \frac{c}{b}\right)_{D}}{\left(\frac{G S D}{k} * \frac{c}{b}\right)_{A}}=\frac{\left(\frac{1}{k} * \frac{c}{b}\right)_{D}}{\left(\frac{1}{k} * \frac{c}{b}\right)_{A}}
$$

The ratios $\mathrm{c} / \mathrm{b}$ are known for a camera, having determined their longitudinal overlap. At first, it is assumed that $k$, an indicator of precision is the same for both cameras. Then, it is determined the empirical $Z$ precision of a digital camera $\left(S_{Z D}\right)$, the empirical $Z$ precision of a film camera $\left(S_{Z A}\right)$ and their ratio:

$$
\frac{\left(\sigma_{z}\right)_{D}}{\left(\sigma_{z}\right)_{A}} \neq \frac{\left(s_{z}\right)_{D}}{\left(s_{z}\right)_{A}} \Rightarrow k_{D} \neq k_{A}
$$

Since $c / b$ ratios are known for the two cameras, $k$ which marks the measurement precision in the image plane is different for the two cameras (the higher $k$, the better precision). The following cases can be obtained:

- Theoretical ratio greater than the empirical one. As a result, the precision achieved in digital camera $Z$ is greater than expected. Therefore, it is assumed that $k_{D}$ is greater than $k_{A}$. This means that, somehow, the quality of the digital camera is better than can be expected theoretically.

$$
\frac{\left(\sigma_{z}\right)_{D}}{\left(\sigma_{z}\right)_{A}}>\frac{\left(s_{z}\right)_{D}}{\left(s_{z}\right)_{A}} \Rightarrow k_{D}>k_{A}
$$

- Theoretical ratio less than the empirical one. It is the opposite of the previous case, so the $\mathrm{Z}$ precision achieved in digital camera is smaller than expected. As a result the quality of the digital camera is worse than the film camera.

$$
\frac{\left(\sigma_{z}\right)_{D}}{\left(\sigma_{z}\right)_{A}}<\frac{\left(s_{z}\right)_{D}}{\left(s_{z}\right)_{A}} \Rightarrow k_{D}<k_{A}
$$

- There are no significant differences between the theoretical and the empirical ratio. There is no difference in the stereoscopic precision.

$$
\frac{\left(\sigma_{z}\right)_{D}}{\left(\sigma_{z}\right)_{A}} \approx \frac{\left(s_{z}\right)_{D}}{\left(s_{z}\right)_{A}} \Rightarrow k_{D} \approx k_{A}
$$

In the case of significant differences between the ratio of theoretical and empirical precision, a significant change in the quality of stereoscopic measurement is obtained, since the geometric basis of the ratio $c / b$ is indisputable. Therefore, only $k$ is an indicator of measurement precision in the image plane. If the measurements are made under the same conditions, the differences can be attributed not to the measurement in the image but the image quality itself. By and large, if significant or important differences are obtained between the empirical and theoretical ratio, a significant difference in image quality could be the reason. 


\subsubsection{Relationship between planimetric and altimetric precision}

The ratio between the planimetric and altimetric precisions obtained, $S_{X Y} / S_{Z}$, compared with the ratio $B / H$, expresses the variation between the planimetric and altimetric precisions. Since, theoretically, this ratio is unity:

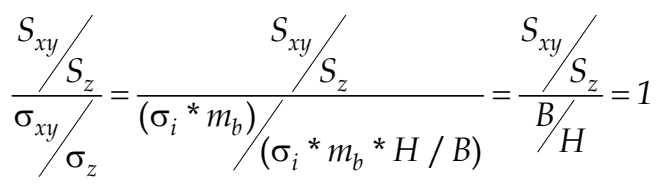

Therefore, if the value for this ratio is less than one, this would indicate that planimetric precision is better than the altimetric precision. Otherwise (greater than one), this camera would show worse results in altimetry than planimetry.

\subsubsection{Comparison of the averages of within-subject measures}

Since we want to establish whether there are differences between the cameras, the right thing is to compare the results based on an operator individually. Keep in mind that the variability between operators may be greater than the variability between the cameras, each operator must be studied independently. In fact, just applying a simple hypothesis test corresponding to the homogeneity of variances, it is possible to observe that there are no significant differences between cameras while there are differences between operators. So, it would be wrong to use the $t$ comparison test for the assessment of the precision of the two cameras, since there are not different operators, but the same operator measures with two different cameras. Therefore, we apply the $t$ test comparison of the averages of withinsubject measures, which should follow a normal distribution with mean zero. The null hypothesis, $H_{0}$, establishes that the different cameras do not affect the precision obtained. The alternative hypothesis, $H_{a}$, establishes that the different cameras modify the precision, and thus the mean difference is not zero.

$$
\begin{aligned}
& H_{0}: \mu d=0 \\
& H_{a}: \mu d \neq 0
\end{aligned}
$$

The statistical test is constructed around the null hypothesis. It consists in comparing the difference average with the theoretical average, which is zero and represents no change. If calculating the difference average, the value obtained in the sample is not zero, the null hypothesis is rejected. That is, if there are differences between the observed and the null hypothesis, it is accepted that there are differences between cameras. Considering that the sample is large $(n>30)$ it is assumed that the dataset follows a normal distribution.

\subsubsection{Analysis of agreement}

The Pearson correlation coefficient, $r$, is usually applied to assess the concordance between the results obtained with different instruments. But this strategy would be incorrect in the present case since this coefficient renders the intensity of the linear association between two measures, and not the degree of agreement between them (Bland \& Altman, 1986). A more correct strategy to measure the concordance is to calculate the Intraclass Correlation 
Coefficient, ICC (Fleiss, 1986). Among the various ICC estimators, two of them are used in this paper: $I C C_{C}$ to measure consistency, and $I C C_{A}$ to measure absolute agreement (Doménech, 2005). Both $I C C_{C}$ and $r$ share the fact that are unable to discriminate a constant difference between two sets of observations, but $I C C_{C}$ is sensitive to proportional differences and $r$, no. The $I C C_{A}$ senses any difference between sets as an inconsistency, independently if this difference is constant, proportional or any other. The lower the value of $I C C_{A}$, the larger the disagreement is.

Considering $i$ subjects and $j$ values for these subjects, in order to calculate the ICC, the total variation (SST) of the $i * j$ observations must be decomposed in three terms: the variation due to subjects (SSS), the variation due to evaluators (SSE) and the residual variation $(S S R)$ :

$$
S S T=S S S+S S E+S S R
$$

With the following degrees of freedom $(d f)$ :

$$
\begin{aligned}
& d f_{T}=i^{*} j-1 \\
& d f_{S}=i-1 \\
& d f_{E}=j-1 \\
& d f_{R}=(i-1)^{*}(j-1)
\end{aligned}
$$

Afterwards, the mean values (MS) are computed dividing the sum of squares (SS) by their corresponding degrees of freedom. The $I C C_{A}$ and the $I C C_{C}$ are calculated according to the following expressions:

$$
\begin{aligned}
I_{C C} & =\frac{i *(M S S-M S R)}{i * M S S+j * M S E+(i * j-i-j) * M S R} \\
I C C_{C} & =\frac{M S S-M S R}{M S S+(j-1) * M S R}
\end{aligned}
$$

In Figure 4 four different cases are outlined, showing a perfect linear relationship $(r=1)$. In the top left, a case total agreement is showed, which is obtained when the valuations $A$ and $\mathrm{B}$ are identical, and therefore the two evaluators values are 1 . In the upper right, a case of constant disagreement is depicted, in which the consistency is 1 whereas the total agreement decreases. At the bottom, are showed the cases of proportional disagreement (left) and proportional and constant disagreement (right), where the difference between evaluators can be observed.

While consistency is behaving as an index of additivity the correlation coefficient is shown as an index of linearity. IC $C_{C}$ and $r$ both have in common a lack of sensitivity to contain a constant difference between two sets of observations, but differ in that $I C C_{C}$ is sensitive to differences of proportional representation. The $I C C_{A}$ provides any difference between measures with disagreement, whether they are of constant rate, proportional or otherwise. The lower the value of $I C C_{A}$, the more disagreement exists. 


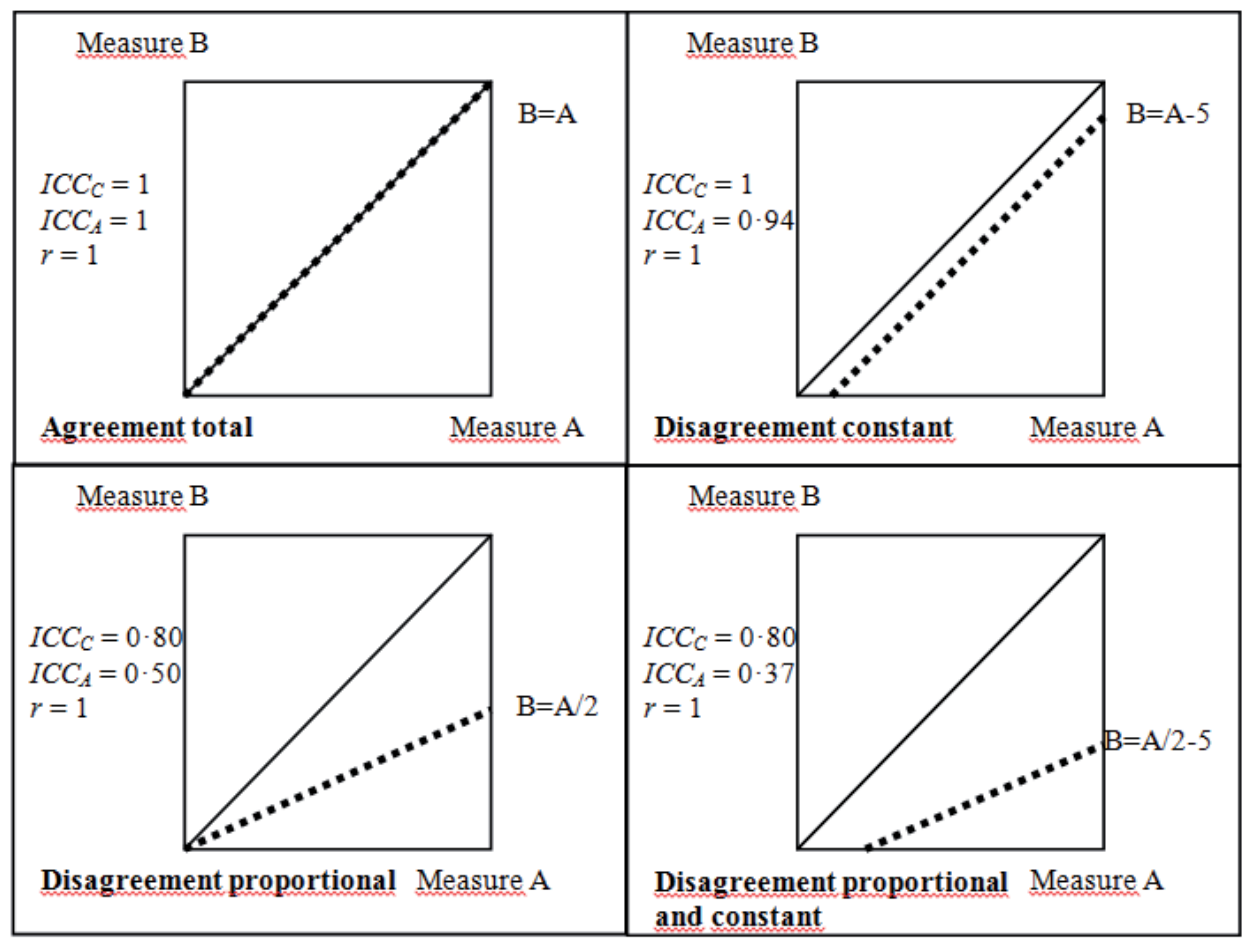

Fig. 4. Consistency, total agreement and linear correlation coefficient (Doménech, 2005).

\subsubsection{Personal equation}

As is known, errors can be classified as instrumental, natural or personal (Wolf \& Ghilani, 1997):

- Instrumental errors: caused by imperfections in the construction or adjustment of the instruments.

- Natural errors: caused by the variation of environmental conditions.

- Personal errors: due to human limitations. The size of this error depends on personal ability and skill of each operator.

Aerial photogrammetry has always coped with the latter type of error in terms of personal equation, assuming this error as a systematic trend of each operator.

Stereoscopic measurements in the personal equation are used to compare film and digital cameras. It is expected that an operator who carries out the measures under the real situation of the point with film camera, do the same with digital camera. Even the relationship between personal equations of film and digital camera should reflect the theoretical ratio $(b / h)$ between the two cameras.

A value for the "real" situation of a point can be calculated as the average of all the observations made by all operators at that point. The personal equation of each operator, $E q_{i}$, can be obtained by the difference of his personal measure with the global measure of all operators (real situation): 


$$
E q_{i}=A-a_{i}
$$

where $i$ is the operator, $A$ is the average of all measurements of all operators to a certain point, and $a_{i}$ is the average of all measurements at that point made by the operator $i$.

Logically, only those measures which imply the same operators observing the same points with film and digital camera will be presented and analyzed.

\subsection{Relative relieves}

The relief range of an area, $\Delta H$, can be defined as the difference between the maximum and minimum height:

$$
\Delta H=Z \max -\mathrm{Zmin}
$$

While the relative relieve is the ratio between the relief of an area and flight height, $H$, from which the images were taken:

$$
\text { Relative relieve }=\frac{\Delta H}{H}
$$

This relative relief indicates a degree of three-dimensional enhancement. The higher the relative relieve is the better is perceived the relief, but it also implies a greater variability in the positioning, and if the operator does not make good measurements, this would explain a larger standard deviation in a series of repeated measures. However, too large relative relieve should get worse the altimetric positioning due to effects of perspective, i.e., there must be an optimum value for which a greater precision is achieved in the altimetric positioning. Anyway, for the flights used in this work, it can be remarked that the higher the relative relieve, the better the stereoscopic positioning.

Considering two different flight heights for the two cameras, $H_{A} \neq H_{D}$, for the same study area, $\Delta H_{A}=\Delta H_{D}$, the relationship between the relieves on the two flights is simplified to the relationship between the two flight altitudes:

$$
\frac{\left(\Delta H / H_{A}\right)}{\left(\Delta H / H_{D}\right)}=\frac{H_{D}}{H_{A}}
$$

Then the ratio of heights of flight gives the variation of relative relieve between the two cameras. The following values can be obtained:

- $H_{D} / H_{A}>1$ : the relieve is noticed worse with the digital camera than with the film camera. This is the situation that occurs in most cases, since for the same GSD, $H_{D}>H_{A}$.

- $H_{D} / H_{A}<1$ : the relieve is noticed better with the digital camera than with the film camera.

- $H_{D} / H_{A}=1$ : the relieve is noticed equal for both cameras.

\section{Experimental results}

This section shows and analyzes the experimental results obtained with three different flights establishing a comparison between the stereoscopic precision obtained with film and 
digital cameras. Aspects such as: type of measurement, operator expertise, point type and area of the stereoscopic model, have been considered.

\subsection{Flights}

The flights used to undertake the stereoscopic measurements performed by operators are:

- "Laguna de Duero" (LD): this large scale flight play an essential role since establishes comparison between film camera (LD_AE) and digital camera (LD_D). In addition, this flight was observed with the original negatives (LD_AA) in an analytical photogrammetric station, Leica SD2000.

- "Mansilla de las Mulas" (MM): this large-scale flight was recorded only with digital camera (MM_D).

- "Arauzo" (AR): this small-scale flight performs as a comparison between film camera (AR_AE) and digital camera (AR_D).

Table 2 collects all these data.

\begin{tabular}{|c|c|c|c|c|c|c|c|c|}
\hline Flight & Camera & $\mathrm{m}_{\mathrm{b}}$ & $\begin{array}{c}\mathrm{px} \\
(\mu \mathrm{m})\end{array}$ & $\begin{array}{c}\mathrm{GSD} \\
(\mathrm{m})\end{array}$ & $\begin{array}{c}\mathrm{B} \\
(\mathrm{m})\end{array}$ & $\begin{array}{c}\mathrm{H} \\
(\mathrm{m})\end{array}$ & $\mathrm{B} / \mathrm{H}$ & $\begin{array}{c}\mathrm{c} \\
(\mathrm{mm})\end{array}$ \\
\hline LD_AE & Leica RC30 & 5.000 & 20 & 0,100 & 450 & 767 & 0,587 & 153,42 \\
\hline LD_D & UltraCamD & 8.333 & 9 & 0,075 & 225 & 845 & 0,266 & 101,40 \\
\hline MM_D & UltraCamD & 11.111 & 9 & 0,100 & 300 & 1,125 & 0,267 & 101,40 \\
\hline AR_AE & Leica RC30 & 30.000 & 15 & 0,450 & 2,686 & 4,600 & 0,583 & 153,42 \\
\hline AR_D & UltraCamD & 55.555 & 9 & 0,500 & 1,500 & 5,633 & 0,267 & 101,40 \\
\hline
\end{tabular}

Table 2. Flight used for manual stereoscopic measurements, where $m_{b}$ indicates the scale denominator of the photographs; $p x$, the pixel size; GSD, the pixel projection over the ground; $B$, the base (ground); $H$, the flight height; $B / H$, the base-height ratio; and $c$, the focal length of the camera.

\subsection{Hypothesis}

\subsubsection{Measurements}

For every point and operator, the standard deviation in $X Y, S_{X Y}$, has been obtained and also the standard deviation in $Z, S_{Z}$. These are parameters to be analyzed and considered to express the precision of the stereoscopic (both planimeric and altimetric) measures, as expressed by Hallert (1959) on repeated direct measurements of unknown quantities.

It is not aimed to asses the global precision of a photogrammetric product but the precision related to the stereoscopic model (Kraus, 1993). The point stereoscopic measurements have been done in this order: first point, second point and so on until the last point to complete one cycle. No point has been observed $n$ times in a consecutive fashion. To achieve $n$ measurements of the same point, the cycle has been repeated $n$ times. Each operator has realized 3 cycles at the beginning of the day and 3 cycles again at midday, to avoid tiredness in his performance, the repeatability in measurements and the so called learning effect. In 
this way, 6 measurements per point and operator have been obtained for a total of 13 operators from public and private companies. These stereoplotter operators are daily engaged in purely stereoscopic photogrammetric procedures (stereoplotting, editing DTMs) and have an experience that ranges from 10 years (high) to 5 years (medium) and to 1-2 years (low). In any case the minimum experience to achieve significant results has been considered to be one year.

Due to the modular composition of large format digital cameras (DMC and UltraCamD) it has been considered relevant to perform a geometric analysis based on the distribution of the points across the stereoscopic area. Consequently, the points have been distributed in nine zones of the stereoscopic model. On each of these nine zones, at least one of the three following types of points has been measured: well defined points on the terrain; easy urban points (roofs) and difficult urban points (ground points close to buildings).

\subsubsection{Types of points}

For the different cases of study three types of points have been considered:

- Well-defined terrain points.

- Well-defined urban points (roofs and curbs, both above and below).

- Difficult urban points (ground points close to buildings).

\subsubsection{Area of the stereoscopic model}

Given the modular structure of the images coming from large-format digital cameras, a geometric analysis has been performed based on the location of points within the stereoscopic model. Therefore, the points shall be distributed in nine areas of the stereoscopic model, to analyze the influence of the position of point in the model. In each one of these areas at least one point of each type will be chosen (Fig. 5).

\begin{tabular}{|l|l|l|}
\hline 1 & 2 & 3 \\
\hline 4 & 5 & 6 \\
\hline 7 & 8 & 9 \\
\hline
\end{tabular}

Fig. 5. Numbering of areas within the stereoscopic model.

\subsection{Case study 1: Large-scale flight Laguna de Duero (LD)}

In the stereoscopic model obtained with the film camera, Leica RC30 (LD_AE), in Laguna de Duero, 46 points have been observed. These measurements were made by five different operators in two sets of three cycles each. The five operators were distributed as follows: 2 with high experience, 1 with medium experience and 2 with low experience: 


\begin{tabular}{|c|c|c|c|}
\hline Operator & $S_{X Y}(m)$ & $S_{Z}(m)$ & Number of observations \\
\hline 1 & 0,013 & 0,025 & 274 \\
\hline 2 & 0,018 & 0,042 & 276 \\
\hline 3 & 0,012 & 0,030 & 268 \\
\hline 4 & 0,020 & 0,033 & 276 \\
\hline 5 & 0,018 & 0,030 & 275 \\
\hline Average & 0,016 & 0,032 & Total 1.369 \\
\hline
\end{tabular}

Table 3. Precision obtained with the flight LD_AE.

These same operators observed the same points in digital images for the Laguna de Duero flight using the UltraCamD digital camera (LD_D):

\begin{tabular}{|c|c|c|c|}
\hline Operator & $S_{X Y}(m)$ & $S_{Z}(m)$ & Number of observations \\
\hline 1 & 0,014 & 0,042 & 274 \\
\hline 2 & 0,018 & 0,070 & 274 \\
\hline 3 & 0,011 & 0,048 & 275 \\
\hline 4 & 0,019 & 0,063 & 276 \\
\hline 5 & 0,017 & 0,053 & 273 \\
\hline Average & 0,016 & 0,055 & Total 1.372 \\
\hline
\end{tabular}

Table 4. Precision obtained with the flight LD_D.

\subsubsection{Film-digital flight comparison: LD_D vs. LD_AE}

The ratio of $S_{X Y}$ for the two flights is unity $(0,016 / 0,016)$, so there are significant differences in planimetry. The ratio of $Z$ precision is determined empirically as $1,719(0,055 / 0,032)$. While the ratio given theoretically is:

$$
\frac{\left(\sigma_{z}\right)_{L D_{-} D}}{\left(\sigma_{z}\right)_{L D_{-} A E}}=\frac{\left(\frac{0,075 m}{k} * \frac{101,4 m m}{27 m m}\right)_{L D_{-} D}}{\left(\frac{0,100 m}{k} * \frac{153,42 m m}{88 m m}\right)_{L D \_A E}}=1,616 * \frac{k_{L D \_} A E}{k_{L D \_D}}
$$

Comparing the observed with the theoretical, it results $k_{L D \_A E}=1,06 * \mathrm{k}_{L D \_D}(6 \%)$. It concludes that there are only slight variations in the precision with digital camera than with the film camera.

Are these empirical dataset concluding? Yes, very concluding, since they encompass 5 operators, with different experience and measuring the same points with film and digital camera. In addition, these points are well distributed throughout the model and represent all types of points.

What might be due this slight difference? The relative relieve is the ratio between the relieve of an area and the flight altitude from which the images were taken $(\Delta H / H)$. The relative ratio between relieves indicates the variation between the relative relieve between both cameras: 


$$
\frac{(\Delta H / H)_{D}}{(\Delta H / H)_{A E}}=0,90
$$

This indicates that the digital flight observes the relieve a $10 \%$ flatter than the film flight. This value may explain the difference of $6 \%$ previously observed.

\subsubsection{Average differences for the $S_{X Y}$ : LD_D vs. LD_AE}

Using the comparison of the averages of within-subject measures introduced in the previous section, Table 5 outlines the comparison of averages for the standard deviation in XY.

\begin{tabular}{|c|c|c|c|}
\hline Operator & Average differences $(\mathrm{mm})$ & $\begin{array}{c}\text { Confidence interval } \\
\text { (lower; upper) }(\mathrm{mm})\end{array}$ & $p$-value \\
\hline 1 & 0,587 & $-3 ; 4$ & 0,771 \\
\hline 2 & $-0,348$ & $-5 ; 4$ & 0,880 \\
\hline 3 & $-0,565$ & $-3 ; 2$ & 0,694 \\
\hline 4 & $-0,652$ & $-6 ; 5$ & 0,813 \\
\hline 5 & $-0,522$ & $-6 ; 5$ & 0,850 \\
\hline
\end{tabular}

Table 5. Comparison of averages for $S_{X Y}$ : LD_D vs. LD_AE. Confidence level of $95 \%$.

In view of the differences in $S_{X Y}$ between the flights LD_D and $L D \_A E$ (operators 1 to 5), one can make the following observations:

- The differences averages are less than a millimeter for all operators and, except for the first one, they are negative, which means that the $S_{X Y}$ of LD_D is larger than the $S_{X Y}$ of LD_AE.

- $\quad$ The $95 \%$ confidence intervals of the differences averages provide interesting ranges. All of them are, approximately, symmetric intervals centred on zero. This is consistent with the fact that there are no really differences between the cameras.

- There are no significant differences for any operator, for a significance level of $5 \%$.

\subsubsection{Average differences for $S_{Z}$ : LD_D vs. LD_AE}

The following table (Table 6) shows the data for comparison of averages for the standard deviation in $Z$.

\begin{tabular}{|c|c|c|c|}
\hline Operator & $\begin{array}{c}\text { Average differences } \\
(\mathrm{mm})\end{array}$ & $\begin{array}{c}\text { Confidence interval } \\
\text { (lower; } \text { upper) }(\mathrm{mm})\end{array}$ & p-value \\
\hline 1 & 17,761 & $11 ; 24$ & 0,000 \\
\hline 2 & 28,739 & $10 ; 47$ & 0,003 \\
\hline 3 & 18,304 & $7 ; 28$ & 0,001 \\
\hline 4 & 29,652 & $19 ; 39$ & 0,000 \\
\hline 5 & 23,587 & $13 ; 33$ & 0,000 \\
\hline
\end{tabular}

Table 6. Comparison of averages for $S_{Z}$ : LD_D vs. LD_AE. Confidence level of $95 \%$. 
In view of the differences in $S_{Z}$ between the flights LD_D and LD_AE (operators 1 to 5), one can make the following observations:

- The differences averages range from $17,761 \mathrm{~mm}$ for the operator 1 to $29,652 \mathrm{~mm}$ for the operator 4 . For all operators, the differences averages are positive, which means that the $S_{Z}$ of LD_D is larger than the $S_{Z}$ of LD_AE.

- $\quad$ None of the $95 \%$ confidence intervals of differences means contains zero.

- There are significant differences in all operators, for a significance level of $5 \%$.

\subsubsection{Analysis of agreement: LD_D vs. LD_AE}

As in the previous case this comparison element can be applied only on the flights that were observed by the same operators. The results are collected in Table 7.

\begin{tabular}{|c|c|c|}
\hline & $\mathrm{S}_{X Y}$ & $\mathrm{~S}_{\mathrm{Z}}$ \\
\hline$I C C_{C}$ & $0,835(\mathrm{p}=0,019)$ & $0,854(\mathrm{p}=0,015)$ \\
\hline$I C C_{A}$ & $0,735(\mathrm{p}=0,019)$ & $0,288(\mathrm{p}=0,015)$ \\
\hline$r$ & $0,893(\mathrm{p}=0,021)$ & $0,988(\mathrm{p}=0,001)$ \\
\hline
\end{tabular}

Table 7. LD_D vs. LD_AE: ICC : consistency; $I_{C C}$ : total agreement; $r$ : linear correlation coefficient, p: p-value: contrast significance of the differences average. Confidence level of $95 \%$.

In case of the data from flights LD_D vs. LD_AE, the relationship between the values of $S_{X Y}$ shows a good agreement, consistent and linear, although there is a slight constant discrepancy. The relationship between the values $S_{Z}$ shows a low agreement but consistent and linear. This indicates that in the relationship between the values of $S_{Z}$ there is a proportionate and constant inconsistency

\subsubsection{Personal equation: LD_D vs. LD_AE}

The personal equation of each operator $Z$ has been calculated for each point, as explained above (expression 21), from the observations performed in LD_D and LD_AE. The average data are shown in Table 8:

\begin{tabular}{|c|c|c|c|}
\hline Operator & LD_D $(\mathrm{mm})$ & $L D_{-} A E(\mathrm{~mm})$ & LD_D/LD_AE \\
\hline 1 & -32 & -16 & 2,0 \\
\hline 2 & 84 & 55 & 1,5 \\
\hline 3 & -109 & -43 & 2,5 \\
\hline 4 & 86 & 21 & 4,1 \\
\hline 5 & -29 & -18 & 1,6 \\
\hline
\end{tabular}

Table 8. Average values of the personal equation for the operators who performed the measurements for LD_AE and LD_D.

Those values in the right column (Table 8) which differ from the unit mean that an operator behaves differently with the two cameras. 
These average values per operator in the personal equation shows that the behavior of an operator in film measures is transmitted to digital measures, reflecting the same sign and a ratio (LD_D/LD_AE) between 4,1 and 1,5.

In order to analyze in detail the personal equation and its variation between cameras, the test of average differences has been applied. The following table (Table 9) summarizes the results of this comparison of paired samples.

\begin{tabular}{|c|c|c|c|}
\hline Operator & $\begin{array}{c}\text { Average differences } \\
(\mathrm{mm})\end{array}$ & $\begin{array}{c}\text { Confidence interval } \\
\text { (lower; } \text { upper })(\mathrm{mm})\end{array}$ & p-value \\
\hline 1 & -16 & $-32 ; 1$ & 0,059 \\
\hline 2 & 29 & $-3 ; 60$ & 0,075 \\
\hline 3 & -66 & $-83 ;-49$ & 0,000 \\
\hline 4 & 64 & $46 ; 82$ & 0,000 \\
\hline 5 & -12 & $-25 ; 2$ & 0,086 \\
\hline
\end{tabular}

Table 9. Test results: comparison of averages differences for the personal equation of the operators who performed observations on flights LD_AE and LD_D. Confidence interval $95 \%$.

In view of the hypothesis testing for differences in personal equation, the following observations can be pointed out:

- The averages differences range from $-66 \mathrm{~mm}$ for the Operator 3 to $64 \mathrm{~mm}$ for the Operator 4. In three cases (operators 1, 3 and 5), the average difference is negative, but this does not mean that the personal equation is greater for LD_D than for LD_AE, but this value is because the personal equation for these operators is negative in both cases (note the data of personal equation for operator in Table 9).

- The confidence intervals at $95 \%$ for the averages differences for the operators 1,2 and 5 contain the zero value, but they are markedly asymmetric, while the intervals of the operators 3 and 4 do not include zero.

- In line with the comments in the previous section, there are no significant differences for a significance level of $5 \%$ for the operators 1, 2 and 5, but they show very low values, which suggests that there are differences. For the other two operators it can be said clearly that there are differences between cameras.

\subsubsection{Other considerations: LD_D vs. LD_AE}

Two more additional considerations should be remarked. The first consideration is based on the location of the points where the measures are performed. In order to get the same ground sample distance (GSD) with film and digital camera, it is necessary to use several digital camera models that cover the same area recorded by a film camera. The second consideration is related with the type of point. Especially in the case of difficult points distributed on the bottom of the buildings, the precision will get worse in $\mathrm{Z}$ for digital camera. 


\begin{tabular}{|c|c|c|c|c|}
\hline Type of point & $\begin{array}{c}S_{X Y \_} L D \_A E \\
(m)\end{array}$ & $\begin{array}{c}S_{X Y \_L D \_} D \\
(m)\end{array}$ & $\begin{array}{c}S_{Z \_} L D \_A E \\
(m)\end{array}$ & $\begin{array}{c}S_{Z \_} L D \_D \\
(m)\end{array}$ \\
\hline$T$ & 0,014 & 0,017 & 0,029 & 0,052 \\
\hline$U \_S$ & 0,015 & 0,014 & 0,033 & 0,050 \\
\hline$U \_C$ & 0,024 & 0,017 & 0,042 & 0,074 \\
\hline
\end{tabular}

Table 10. Precision obtained with flights LD_D and LD_AE, with different types of points: T: well-defined terrain points; $U \_S$ : Well-defined urban points; $U \_C$ : Difficult urban points.

These two considerations suggest that the measures will not be performed equally for both cameras.

What would it happen if the scanning resolution for film images were $15 \mu \mathrm{m}$ instead of 20 $\mu \mathrm{m}$ ? In this case the pixel size on the ground for the film camera would be $0,075 \mathrm{~m}$. A value similar to the digital flight, so that the theoretical ratio of altimetric precision would be as follows:

$$
\frac{\left(\sigma_{z}\right)_{L D \_D}}{\left(\sigma_{z}\right)_{L D \_A A}}=\frac{\left(\frac{0,075 m}{k} * \frac{101,4 m m}{27 m m}\right)_{L D \_D}}{\left(\frac{0,075 m}{k} * \frac{153,42 m m}{88 m m}\right)_{L D \_A A}}=2,155 * \frac{k_{L D \_A A}}{k_{L D \_D}}
$$

This value of 2,155 is very different (about 33\%) from its theoretical, 1,616. Nevertheless, if these same 5 operators perform their measures with a $15 \mu \mathrm{m}$ image, do the empirical precision would be $33 \%$ better? Several authors believe that the optimal size is $15 \mu \mathrm{m}$ scan (Boniface, 1996). The next section addresses this question.

Note that for changing the GSD size (15 to $20 \mu \mathrm{m}$ ) with film camera is not necessary to change any parameters of the proposed flight, but simply the scanning pixel size. Neither the focal length or the frame size or resolution of the film, etc.

\subsubsection{Comparison of: LD_AE vs. LD_AA}

In this case, manual measures were performed over the same points considering the original images, using a Leica SD2000 analytical plotter with analog vision system, but with other operators in the restitution. This is the flight LD_AA.

\begin{tabular}{|c|c|c|c|}
\hline Operator & $S_{X Y}(m)$ & $S_{Z}(m)$ & Number of observations \\
\hline 1 & 0,012 & 0,021 & 275 \\
\hline 2 & 0,019 & 0,031 & 269 \\
\hline 3 & 0,012 & 0,020 & 276 \\
\hline 4 & 0,011 & 0,027 & 274 \\
\hline Summary & 0,014 & 0,025 & 1094 \\
\hline
\end{tabular}

Table 11. Precision obtained with the flight LD_AA. 
The $X Y$ precision ratio shows a decrease of $14 \%(0,016 / 0,014)$ with the image scanned at 20 $\mu \mathrm{m}$.

This may indicate that a pixel scan size of $17,5 \mu \mathrm{m}(20 / 1,14)$ would have been optimal. The results come to confirm that with a scanned resolution of $20 \mu \mathrm{m}$ the $Z$ precision is about $28 \%$ $(0,032 / 0,025)$ worse than with the original images. According to this data and considering that the scan size is the main factor, the optimal size would have been of $15,6 \mu \mathrm{m}(20 / 1,28)$. (In this case, the theoretical reason $\sigma_{Z}$ is 1 since it is the same camera).

These data seem to indicate that more precision could have been obtained if the images had been scanned at $15 \mu \mathrm{m}$. With this, the GSD $(0,10 \mathrm{~m})$ would have been the same for the film and digital camera.

Note that the measure instrument is completely different (including the stereoscopic system). In addition, measures were made with different operators. Probably, those differences encountered during the experiments could be related with these variables.

\subsubsection{Comparison: LD_D vs. LD_AA}

If we work with global average values for both flights, a comparison can be established, even knowing the differences between measurements (different instrument and different operators). The $X Y$ precision ratio is observed to be about $14 \%(0,016 / 0,014)$ more accurate for film flight. The theoretical precision in Z, considering the same GSD for both flights, is of 2,155 , while the empirical precision is $2,200(0,055 / 0,025)$, almost the same.

\subsection{Case study 2: Large-scale flight Mansilla de las Mulas (MM)}

Due to initial requirements for the selection of points and the type of points, it is not clear the precision related with those flights executed with low height. For this purpose, several measures were performed for a digital flight, MM_D, using a different collection of points and with a $0,10 \mathrm{~m}$ GSD. In this case, only well-defined terrain points were observed, distributing three points along the nine areas of the stereoscopic model. The operators were four: 2 with high experience, 1with medium experience and 1 with low experience (Table 12).

Comparing LD_AE vs. MM_D, the difference for XY precision reaches the $13 \%$ $(0,018 / 0,016)$, when the GSD is the same. The ratio for $Z$ precision is determined empirically as $1,594(0,051 / 0,032)$, while the ratio determined theoretically, having the same GSD, is 2,155. As a result, the digital flight MM_D is a 35\% better than the film flight LD_AE ( $k_{M M} D$ $\left.=1.35 * k_{L D_{-} \mathrm{AE}}\right)$ considering a pixel size of 20 microns.

\begin{tabular}{|c|c|c|c|}
\hline Operator & $S x y(m)$ & $S z(m)$ & Number of observations \\
\hline 1 & 0,016 & 0,053 & 162 \\
\hline 2 & 0,018 & 0,046 & 162 \\
\hline 3 & 0,022 & 0,062 & 162 \\
\hline 4 & 0,016 & 0,044 & 162 \\
\hline Average & 0,018 & 0,051 & Total 808 \\
\hline
\end{tabular}

Table 12. Precision obtained with the flight MM_D. 
Comparing LD_AA vs. MM_D, the difference in XY precision is $28 \%(0,018 / 0,014)$, while the $\mathrm{Z}$ empirical precision is $2,040(0,051 / 0,025)$, pretty much the same than the $\mathrm{Z}$ theoretical precision, 2,155.

\subsection{Comparison between large-scale flights}

The following table (Table 13) shows a series of data representative of each flight which allow us to make comparisons between flights.

\begin{tabular}{|l|c|c|c|c|}
\hline & $\begin{array}{c}\text { LD_AE } \\
\text { RC30-D }\end{array}$ & $\begin{array}{c}\text { LD_AA } \\
\text { RC30-A }\end{array}$ & $\begin{array}{c}\text { LD_D } \\
\text { ULC-D }\end{array}$ & $\begin{array}{c}\text { MM_D } \\
\text { ULC-D }\end{array}$ \\
\hline$S_{i}(\mu \mathrm{m})$ & 3,20 & 2,80 & 1,92 & 1,62 \\
\hline$p x(\mu m)$ & 20 & 15 & 9 & 9 \\
\hline$K$ & 6,25 & 5,37 & 4,69 & 5,56 \\
\hline$S_{X Y} / G S D$ & 0,16 & 0,19 & 0,21 & 0,18 \\
\hline$S_{Z} / H\left(x 10^{-5}\right)$ & 4,17 & 3,26 & 6,53 & 6,03 \\
\hline$\left(S_{X Y} / S_{Z}\right) /(B / H)$ & 0,85 & 0,95 & 1,09 & 1,32 \\
\hline
\end{tabular}

Table 13. Data coming from the large-scale flights analyzed.

In the table 13: Si: image measures precision in micrometers, $p x$ : pixel size in microns; $k$ : precision indicator; $S_{X Y} / G S D$ : ratio between planimetric precision and GSD, $S_{Z} / H$ : ratio between altimetric precision and flight height; $\left(S_{X Y} / S_{Z}\right) /(B / H)$ quotient between the planimetric and height empirical standard deviations ratio and the planimetric and height theoretical precision ratios. Note that it has been assumed that the $p x$ of LD_AA, the film flight observed with the analytical stereoplotter, is $15 \mu \mathrm{m}$.

The values of $S_{i}$ and $p x$ are not comparable, whereas the indicators of precision, $k$ are comparable. It must be emphasized the equality between MM_D and LD_AA. The minor value for the digital camera flight LD_D could be related with the selection and type of points, while best values reached for film camera flight LD_AE, may be due to the pixel size of $20 \mu \mathrm{m}$. The ratios $S_{X Y} / G S D$ are similar for all flights. The ratios $S_{Z} / H$ for film camera flights are around $3-4^{*} 10^{-5}(0,00003)$, while for the two digital flights are around $6^{*} 10^{-5}$.

For both film flights the quotient $\left(S_{X Y} / S_{Z}\right) /(B / H)$ indicates that the planimetric precision is better than the altimetric precision, whereas for the digital flights the quotient expresses the opposite.

\subsection{Case study 3: Small-scale flight Arauzo}

A case of study was performed in Arauzo (Spain), using a small-scale and combining film camera (AR_AE) and digital camera (AR_D). In particular, for the film flight, AR_AE, 3 points were measured along the 9 areas of the stereoscopic model (a total of 27 points). The points were observed by four different operators in 2 sets of 3 cycles each. The measured points were well-defined terrain points, since the workspace was rural. The experience of the operators was distributed as follows: 2 with high experience, 1 with medium experience and 1 with low experience. Table 14 outlines the main results. 


\begin{tabular}{|c|c|c|c|}
\hline Operator & $S_{X Y}(m)$ & $S_{Z}(m)$ & Number of observations \\
\hline 1 & 0,093 & 0,132 & 162 \\
\hline 2 & 0,093 & 0,125 & 162 \\
\hline 3 & 0,104 & 0,214 & 161 \\
\hline 4 & 0,084 & 0,119 & 162 \\
\hline Summary & 0,094 & 0,148 & 647 \\
\hline
\end{tabular}

Table 14. Precision obtained with the film flight AR_AE.

The digital flight AR_D was observed by these same four operators, which measured the same points.

\begin{tabular}{|c|c|c|c|}
\hline Operator & $S_{X Y}(m)$ & $S_{Z}(m)$ & Number of summary \\
\hline 1 & 0,124 & 0,228 & 162 \\
\hline 2 & 0,086 & 0,221 & 161 \\
\hline 3 & 0,145 & 0,311 & 162 \\
\hline 4 & 0,110 & 0,229 & 162 \\
\hline Summary & 0,116 & 0,247 & 647 \\
\hline
\end{tabular}

Table 15. Precision obtained with the digital flight AR_D.

\subsubsection{Comparison between film and digital flight: AR_AE vs. AR_D}

Comparing the precision obtained, it can see that the planimetric ratio $S_{X Y}(0,116 / 0,094)$ is $23 \%$ worse with the digital camera, even when the difference between GSD is only $11 \%$ $(0,500 / 0,450)$. It is important to point out the uncertainty in the point definition given the rustic area. The ratio of $Z$ precision is determined empirically as $1,669(0,247 / 0,148)$, while the ratio if $Z$ precision determined theoretically is 2,393 . This implies that $k_{A R_{-} D}=1,43$ * $k_{A R \_A E}$ :

$$
\frac{\left(\sigma_{z}\right)_{A R_{\_} D}}{\left(\sigma_{z}\right)_{A R_{\_} A E}}=\frac{\left(\frac{0,500 m}{k} * \frac{101,4 m m}{27 m m}\right)_{A R_{-} D}}{\left(\frac{0,450 m}{k} * \frac{153,42 m m}{88 m m}\right)_{A R_{-} A E}}=2,393 * \frac{k_{A R_{-} A E}}{k_{A R_{-} D}}
$$

It concludes that the precision in $Z$ with the digital camera is better $(43 \%)$ than the precision in $Z$ determined with the film camera. The variation of relative relieve between flights is $22 \%$. The lower relative relieve, the lower range of variability in $Z$ positioning, so that the standard deviation will be less. Also, the difference in relative relieve must be considered in relation to the different focal distances. The longer the focal length the flatter is the relieve.

Note that one important factor which could be minimizing the effect of the ratio $\mathrm{B} / \mathrm{H}$ is the increase of flight height.

The following table (Table 16) collects representative data for each flight: 


\begin{tabular}{|l|c|c|}
\hline & AR_A & AR_D \\
\hline$S_{i}(\mu m)$ & 3,13 & 2,09 \\
\hline$P x(\mu m)$ & 15 & 9 \\
\hline$K$ & 4,79 & 4,31 \\
\hline$S_{x y} / G S D$ & 0,21 & 0,23 \\
\hline$S_{z} / H\left(x 10^{-5}\right)$ & 3,22 & 4,38 \\
\hline$\left(S_{x y} / S_{z}\right) /(B / H)$ & 1,09 & 1,76 \\
\hline
\end{tabular}

Table 16. Data from the four flights analyzed large-scale.

The ratio $S_{X Y} / G S D$ is not very different for both flights $(0,21$ and 0,23$)$, as well as the altimetric ratio $S_{Z} / H\left(3,22^{*} 10^{-5}\right.$ and $\left.4,38^{*} 10^{-5}\right)$. The quotient $\left(S_{X Y} / S_{Z}\right) /(B / H)$ show for both flights that the planimetric precision is lower than the altimetric precision, even though in the case of digital camera is much more pronounced this difference.

\subsubsection{Average differences for $S_{X Y}: A R \_D$ vs. AR_AE}

The following table (Table 17) shows the average differences for $S_{X Y}$ :

\begin{tabular}{|l|l|l|l|}
\hline Operator & $\begin{array}{l}\text { Average diferences } \\
(\mathrm{mm})\end{array}$ & $\begin{array}{l}\text { Confidence interval } \\
\text { (lower; } \text { upper })(\mathrm{mm})\end{array}$ & -value \\
\hline 1 & 30,444 & $-23 ; 84$ & 0,255 \\
\hline 2 & $-7,000$ & $-32 ; 18$ & 0,574 \\
\hline 3 & 40,778 & $-4 ; 85$ & 0,074 \\
\hline 4 & 26,222 & $-27 ; 79$ & 0,322 \\
\hline
\end{tabular}

Table 17. Comparison of averages for $S_{X Y}$ : AR_D vs. AR_AE. Confidence level of $95 \%$.

The following observations can be made for $S_{X Y}$ according to the hypothesis testing:

- For the operators 1, 3 and 4 the average difference is positive, this implies that for these operators $S x y \_A R \_D>S x y \_A R \_A E$. This is not the case for the operator 2 since its performance is opposite.

- The confidence interval $95 \%$ for the average differences containing zero, but show a clear asymmetry.

- For a significance level of 5\%, no significant differences were found for any operator.

\subsubsection{Average differences for $S_{Z}$ : AR_D vs. AR_AE}

The following table (Table 18) shows the average differences for $\mathrm{S}_{\mathrm{Z}}$.

\begin{tabular}{|c|c|c|c|}
\hline Operator & Average differences $(\mathrm{mm})$ & $\begin{array}{c}\text { Confidence interval } \\
\text { (lower; } \text { upper) }(\mathrm{mm})\end{array}$ & -value \\
\hline 1 & 95,630 & $61 ; 129$ & 0,000 \\
\hline 2 & 95,481 & $48 ; 142$ & 0,000 \\
\hline 3 & 97,037 & $33 ; 161$ & 0,004 \\
\hline 4 & 110,111 & $75 ; 145$ & 0,000 \\
\hline
\end{tabular}

Table 18. Comparison of averages for $S_{Z}$ : AR_D vs. AR_AE. Confidence level of $95 \%$. 
According to the hypothesis testing, the following observations can be made for $S z$ :

- For all operators, the average difference is positive, this implies that $S z_{-} R \_D>S z_{-}$ $A R \_A E$. In addition, the average differences are between $95,481 \mathrm{~mm}$ and $110,111 \mathrm{~mm}$.

- None of the confidence intervals of $95 \%$ for the average difference contains zero. All ranges implies that $S z \_A R \_D>S z \_A R \_A E$.

- There are significant differences in all operators, for a significance level of $5 \%$.

\subsubsection{Analysis of agreement: AR_D vs. AR_AE}

The total agreement, the consistency and the correlation coefficient are depicted in the Table 19.

\begin{tabular}{|c|c|c|}
\hline & $S_{X Y}$ & $S_{Z}$ \\
\hline$I C C_{C}$ & $0,620(\mathrm{p}=0,132)$ & $0,940(\mathrm{p}=0,009)$ \\
\hline$I C C_{A}$ & $0,347(\mathrm{p}=0,132)$ & $0,295(\mathrm{p}=0,009)$ \\
\hline$r$ & $0,658(\mathrm{p}=0,171)$ & $0,986(\mathrm{p}=0,007)$ \\
\hline
\end{tabular}

Table 19. AR_D vs. AR_AE: ICC : consistency; ICC A $_{\text {: }}$ total agreement; $r$ : linear correlation coefficient. Confidence level of $95 \%$.

Note that $S_{X Y}$ values are not significant for a confidence level of $95 \%$. On the other hand, the relationship between $S_{Z}$ shows a low total agreement but following a consistent and linear trend.

\subsubsection{Personal equation: AR_D vs. AR_AE}

The following table (Table 20) shows the personal equation results for each operator and based on the observations performed for flights: AR_AE and AR_D.

The values provided by personal equation do not show a clear trend. Even their heterogeneity might indicate that the data are unreliable. In particular, for the operator 1 and 3 the sign is changed, indicating that the altimetric positioning is made above the terrain for digital flights and bellow the terrain for film flights. However, it is important to remark that the average altimetric position of a point is obtained from the average measures of all operators, so that the change of sign should be interpreted as an increase in the personal equation difference between film and digital camera.

\begin{tabular}{|c|c|c|c|}
\hline Operator & AR_D $(\mathrm{mm})$ & AR_AE $(\mathrm{mm})$ & AR_D/AR_AE \\
\hline 1 & 90 & -20 & $-4,5$ \\
\hline 2 & -32 & -64 & 0,5 \\
\hline 3 & -279 & 12 & $-23,5$ \\
\hline 4 & 222 & 72 & 3,1 \\
\hline
\end{tabular}

Table 20. Personal equation for the operators who performed the measurements in flights: AR_D and AR_AE. 
The following table (Table 21) summarizes the results of this comparison of differences for personal equations for each operator and for each point.

\begin{tabular}{|c|c|c|c|}
\hline Operator & Average $(\mathrm{mm})$ & $\begin{array}{c}\text { Confidence interval } \\
\text { (lower; } \text { upper) }(\mathrm{mm})\end{array}$ & $\mathrm{p}$-value \\
\hline 1 & 110 & $49 ; 110$ & 0,001 \\
\hline 2 & 31 & $-22 ; 85$ & 0,236 \\
\hline 3 & -291 & $-386 ;-196$ & 0,000 \\
\hline 4 & 150 & $62 ; 237$ & 0,002 \\
\hline
\end{tabular}

Table 21. Test results comparing average differences for the personal equation of the operators who made observations on flights AR_D and AR_AE. Confidence level of 95\%.

For a significance level of $5 \%$, differences between cameras are found for the operators 1, 3 and 4, whereas no differences are found for the operator 2.

\section{Conclusions}

In this chapter, an assessment of stereoscopic precision for large-format digital cameras has been studied. In all the flights, the influence of a set of variables on the precision in $X Y$ and in $Z$ has been analyzed. These variables are: the distribution of points in the model; the operators and their experience; and the type of points.

In particular, in every case the planimetric ratio $S_{X Y} / G S D$ indicate that the planimetric error is similar for both flights. Besides this, it is supposed that the flight height is independent, even for large flight heights. The altimetric error seems to indicate that the main difference comes form the flight height and in minor level from the relation $b / h$. No significant differences are observed in the ratio $S_{Z} / H$ for all flights analyzed.

In conclusion, the approach of relating digital and film flights through the GSD is right on the planimetric side. However, the altimetric precision must be analyzed carefully in order to determine if it is possible to maintain traditional flight heights. In this sense, the indicators of precision $k$, as a fraction of the pixel of the image, are a good comparator.

The values for the quotient $\left(S_{X Y} / S_{Z}\right) /(B / H)$ show that those film flights provide slightly better results for planimetry than for altimetry, while in the case of digital camera just the opposite occurs, having worse planimetric precision.

The empirical conclusion that seems to be reached is that the negative impact on altimetric precision caused by the lower ratio $\mathrm{B} / \mathrm{H}$ for digital camera flights is lower when the flight height increases.

In summary, the main conclusion to be drawn on the stereo manual measurements is that the planimetric accuracy is the same for both cameras: film and digital. However, altimetric precision does not provide the same results, being the ratio $b / h$ the main cause of the difference between both cameras.

\section{References}

Alamús, R., Kornus, W., Palà, V., Pérez, F., Arbiol, R., Bonet, R., Costa, J., Hernández, J., Marimon, J., Ortiz, M.A., Palma, E., Pla, M., Racero, S., Talaya, J. (2005). Validation 
process of the ICC digital camera. Proceedings of ISPRS Workshop on High-Resolution Earth Imaging for Geospatial Information, Hannover (Germany), May 2005.

Arias, B. \& Gómez-Lahoz, J. (2009). Testing the stereoscopic precision of a large-format digital camera. The Photogrammetric Record, Vol. 24, Issue 126 (June 2009), pp 157170, ISSN 1477-9730.

Blachut, T.J. (1955). General report of commission IV Subcommission IV/3. Small scale mapping. Photogrammetria, Vol. 12 (1955), pp 220-229, ISSN 0031-8663.

Bland, M. \& Altman, D.G. (1986). Statistical methods for assessing agreement between two methods of clinical measurement. Lancet, Vol. i (1986), pp 307-310, ISSN 0140-6736.

Boniface, P. R. (1996). State-of-the-art in SoftCopy Photogrammetry, in: Digital Photogrammetry: An Addendum to the Manual Photogrammetry, C. Greve, American Society of Photogrametry and Remote Sensing, ISBN 978-1570830372, Bethesda, EEUU.

Commission C., O.E.E.P.E. (1955). Zeitlicher Ablauf der Messungen Deroulment chronologique des observations. Photogrammetria, Vol. 12 (1955), pp 198-199, ISSN 0031-8663.

Dörstel, C. (2003). DMC - Practical experiences and Photogrammetric System Performance, Proceedings of Photogrammetric Week, pp 59-65, Stuttgart (Germany), 2003.

Doménech, J.M. (2005) Medida del cambio: Análisis de diseños con medidas intrasujeto. Medida de la Concordancia. Fundamentos de diseño y estadística, 14. Signo, ISBN 9788480493017, España.

Dubuisson, B. (1955) Rapport général de la commission IV Sous-Commission. IV/2 Etablissement des plans des territoires urbains. Photogrammetria, , Vol. 2 (1955), pp 208-219, ISSN 0031-8663.

Fleiss, J.L. (1986). The design and analysis of clinical experiments. John Wiley \& Sons, ISBN 978-0471820475, New York, EEUU.

Förstner. (1955). O.E.E.P.E., Commission C Rapport sur la restitution effectuée dans l'Institut für Angewandte Geodäsie, Francfort sur le Main Terrain d'essai d'Oberriet les vols Nr 1 et 3 (groupe I). Photogrammetria, , Vol. 12 (1955), pp 183-190, ISSN 0031-8663.

Gotthardt, E. (1955). O.E.E.P.E., Commission C Compte rendu de la restitution à la Technischen Hochschule, Stuttgart, des vols d'essai du gruope I du terrain d'Oberriet. Photogrammetria, , Vol. 12 (1955), pp 163-171, ISSN 0031-8663.

Gotthardt, E. (1958). Report on the First Results of Photogrammetric Test Performed near "Oberriet" by Commission C of the O.E.E.P.E. Photogrammetria, , Vol. 15 (1958), pp 141-148, ISSN 0031-8663.

Grifoni, B. (1949) On different grade of precision attained in plotting using glass plate and film cameras. Photogrammetria, , Vol. 6 (1949), pp 55-58, ISSN 0031-8663.

Härry, H. (1954). La situation actuelle dans la mensuration cadastrale photogrammétrique. Photogrammetria, , Vol. 11 (1954), pp 45-50, ISSN 0031-8663.

Härry, H. (1955). Rapport général de la Commission IV Sous-Commission IV/1. Application de la photogrammétrie aux mensurations cadastrales et aux remaniements parcellaires. Photogrammetria, , Vol. 12 (1955), pp 203-207, ISSN 0031-8663.

Karara, H.M. (1967). Mono versus stereo analytical photogrammetry-theoretical considerations and experimental results. Photogrammetria, Vol. 22, Issue 3 (March 1967), pp 99-113, ISSN 0031-8663. 
Krakau, C.E.T. (1970). A device for the automatic. Continuous recording of stereoscopic visual acuity. Photogrammetria, , Vol. 25, Issue 4 (February 1970), pp 115-123, ISSN 0031-8663.

Kraus, K., (1993). Photogrammetry Vol. 1. Fundamentals and standard processes (4th edition), Dümmler, ISBN 3427786846, Bonn, Germany.

Lehmann, G. (1955). Report on the work hitherto archieved by Commission C of the O.E.E.P.E. Photogrammetria, , Vol. 12 (1955), pp 156-163, ISSN 0031-8663 .

O'Connor, D. (1967). Some factors affecting the precision of coordinate measurements on photographic plates. Photogrammetria, Vol. 22, Issue 3 (March 1967), pp 77-97, ISSN 0031-8663.

Schiewe, J. (1995). Cartographical Potential of MOMS-02/D2 Image Data. In: Photogrammetric Week'95. Heidelberd: Ed. Fritsch/Hobbie, Wichmann. pp 99-105.

Spreckels, V., Syrek, L., Schlienkamp, A. (2010). DGPF Project: Evaluation of Digital Photogrammetric Camera Systems - Stereoplotting. Photogrammetrie Fernerkundung - Geoinformation, Vol. 2010, No. 2 (May 2010), pp. 117-130, ISSN 14328364.

Stark, E. (1975). The effect on angular field on horizontal and vertical accuracy in photogrammetric plotting. Procedings of Photogrammetric Week, pp 129-145, Stuttgart (Germany), 1975.

Stickler, C.A. (1959). Interpretation of the results of the O.E.E.P.E. commission C. Photogrammetria, , Vol. 16 (1959), pp 8-12, ISSN 0031-8663.

Stoch, L. (1961). Note on C. A. Stickler's Paper. Photogrammetria, Vol. 18 (1961), pp 34, ISSN 0031-8663.

Trinder, J.C. (1986). Potentials of monocular and stereoscopic observations on aerial photographs. Photogrammetria, Vol. 41, Issue 1 (October 1986), pp 17-27, ISSN 00318663.

Wolf, P.R. \& Ghilani, C.D. (1997). Adjustment computations: statistics and least squares in surveying and GIS. Ed. John Wiley and Sons,ISBN 0471168335, New York, EEUU.

Zorn, H. C. (1965). An instrument for testing stereoscopic acuity. Photogrammetria, Vol. 20, Issue 6 (December 1965), pp 229-238, ISSN 0031-8663. 


\title{
Application of a Photogrammetric System for Monitoring Civil Engineering Structures
}

\author{
Junggeun Han ${ }^{1,}{ }^{*}$, Kikwon Hong 2 and Sanghun Kim ${ }^{3}$ \\ ${ }^{1}$ School of Civil and Environmental Engineering, \\ Urban Design and Study,Chung-Ang University, Seoul \\ ${ }^{2}$ Green Technology Institute, Chung-Ang University, Seoul \\ ${ }^{3}$ ReStl Designers, Inc., Maryland \\ 1,2South Korea \\ ${ }^{3} \mathrm{USA}$
}

\section{Introduction}

Several traditional measuring apparatus are used to check the stability of civil engineering structures and maintenance of them. The measured results are applied for the deformation and stability analysis of civil engineering structures. Currently, precision and micro measuring instruments are used for stability evaluations of civil engineering structures. Furthermore, the measuring apparatus have been changed from manual systems to automatic systems. For example, total station, one of the traditional and manual measuring methods, is transferred to digital photogrammetry with high technology development. Especially, the movement of target points is able to be measured in real-time automatically because it can be obtained 3-dimensional coordinates by digital photogrammetry. The use of automatic measuring methods has been researched in several different industries (Hannah, 1989; Lee et al., 2006). The applications of digital photogrammetry are increased in various civil engineering structures (Han et al., 2001; Han \& Song, 2003; Han et al., 2007, 2008). It shows that the automatic and high-tech measuring system likeVisual Monitoring System (VMS) based on digital photogrammetry is able to apply to the stability evaluations of large civil engineering structures.

Most of recent measurement methods are based on image process method even though in some case, Global Positioning System (GPS) is used like the measurement of the deformation on the surface of large civil engineering structures (Stewart \& Tsakiri, 2001). Also, automatic measuring method begins to use for stability evaluations of civil engineering structures; for examples, there are slope failure prediction (Han et al., 2001; Han \& Song, 2003), displacement measurement (Bae, 2000; Kang et. al, 1995), monitoring of dams(Park et. al 2001; Han et. al, 2005). However, the current measuring systems like total station based on manual measuring are almost not possible to measure the movements of

${ }^{*}$ Corresponding Author 
civil engineering structures in real-time based due to various field conditions even though most of measuring systems have a high accuracy. For example, the manual measuring method requires a lot of analyzing time for measured data because it has several procedures like photographing, drawing and analysis manually.

In the late 21th century, the image acquisition apparatus with high technology has been developed continuously and rapidly. If real-time automatic measuring system based on high technology is developed, the measuring system technique can be developed with digital image technique rapidly as well. It means that stability and maintenance technique for civil engineering structure can be more epochal improved in the future.

Therefore, this chapter introduces a real-time measuring system, VMS which is based on digital photogrammetry. The measuring results of VMS are compared with those of manual apparatus in various tests and then the accuracy of VMS is evaluated. At first, background of digital photogrammetry and VMS process are explained. Secondly, laboratory and field tests using VMS and cultural assets restoration based on application of digital photogrammetry are described. Finnally, conclusions about various tests and case study are summarized.

\section{Background of digital photogrammetry}

\subsection{Historical background}

The past photogrammetry requires several steps to obtain the adequate results which are divided into photographing, drawing, analysis, 3-dimensinal coordinates acquisition and conversion, because it uses films that have sensitive emulsion. Therefore, the past photogrammetry has problems in both the application of real-time measuring and distortion and storage of data.

In the late 20th century, CCD camera was introduced as image acquisition apparatus because CCD camera could not only be economically purchased but also, easily process and save the image data. The image acquisition apparatus has been developed in high resolution and wide analysis range based on the development of electric and electron. Also, the accuracy of camera lens has been improved by correction technique of geometric distortion and establishment of analysis algorithm (Fraser, 1997).

In the early 1990s, the image analysis techniques with film scan started to apply to various fields. In the mid-1990s, several researchers have studied the improvement of accuracy in image analysis techniques using establishment of analysis algorithm and commercialization of real-time measuring (El-Hakim \& Wong, 1990). Furthermore, the 3-dimensional position measuring has been researched to apply the field of photogrammetry (Fraser, 1993; Shortis \& Fraser, 1998). The correction of multi-focus lens has been studied to apply in close range photogrammetry (Munjy, 1985; 1986). The lens correction and image analysis improvement of CCD camera have been studied by using research results (Shortis et al., 1994) of close range photogrammetry and focus lens (Lichti \& Chapman, 1995). CCD camera has been used in image analysis of robot vision and position decision of targets (Gruen, 1992).

The study of combination programs has been a trend, so separate image processing programs could be combined by using visual analysis and connection analysis based on existing researches. Especially, the photogrammetry that uses automatic technique of 3dimensional data acquisition has been studied in the application of object movement and 
automatic system of industrial (Hannah, 1989; Lee et al., 2006). Also, the user interface design using digital photogrammetry technique was able to apply in high accuracy measuring system and vision system.

The 3-dimensional measuring system based on existing photogrammetry is used in film with sensitive emulsion. However, the technique takes a time to respond to the risk in spite of great accuracy. Therefore, real-time measuring system, which improved measuring system based on existing photogrammetry, was requested for civil engineering structure that specially requires both economical, rapid reaction and high accuracy.

\subsection{Short-overview of digital photogrammetry}

Photogrammetry provides the 3-dimensinal coordinates of unknown points from acquired multiple images, which are images of the same scene on different viewpoints. Photogrammetry procedure has object decision, image acquisition, image processing and analysis. The image acquisition of the past photogrammetry is used in film with sensitive emulsion. Nowadays, those are used in CCD and CMOS cameras. The 3-dimensinal coordinate precision of images depends on various factors like object condition, camera calibration and image orientation. That is, the scale and pixel size of image based on CCD and CMOS cameras is important factor in the image acquisition of photogrammetry. This means that camera calibration needs to enhance image accuracy (Kraus, 1993; 1997; 2007).

In present, the digital images are produced by electric sensor of metric cameras based on CCD or CMOS, as above mentioned. The mathematical-geometric parameters of digital image based on principal distance, coordinates and principal point are defined as the factors of interior orientation. Even though geometric parameters represent the photogrammetry analysis model, it does not correspond to real object due to distortion of camera lens. Therefore, the camera calibration must be conducted to achieve the highest accuracy of model (Kraus, 1993; 1997).

In order to create 3-dimensinal coordinates of image, the image plane is produced by electric sensor in focal plane of cameras. The orientation parameters based on image plane can be calculated using collinearity equations (see Kraus, 1993) and then the 3-dimensinal coordinates are produced (Kraus, 1993; 1997; 2007; Ohnishi et al., 2006).

\section{Visual Monitoring System (VMS)}

VMS is monitoring system using digital photogrammetry and it is made to analyze deformation of object based on real-time visualization. This section introduces composition, principle and process of VMS.

\subsection{Composition of VMS}

The past photogrammetry using films makes a 3-dimensinal image by image acquisition, drawing, analysis and 3-dimensinal coordinate creation. A coordinate conversion of film image is conducted by coordinate formation program. It results in conversion process and visualization program and then it forms 3-dimensinal coordinates. The past photogrammetry has a problem for an immediate maintenance about deformation and displacement of structure in field because it requires a lot of processing time. If disaster risk happened, it would require a lot of time to establish countermeasure. 
The VMS photogrammetry analysis software which combine with 3-dimensional coordinate extraction, drawing and analysis, can analyze structural deformation as shown in Fig. 1 . The VMS as a unified program is real-time measuring system for civil engineering structure with using digital photogrammetry. VMS can evaluate the structural stability by comparing the continuous obtained information to the existing information of structure. Also, it is real-time monitoring system for maintenance of structure.

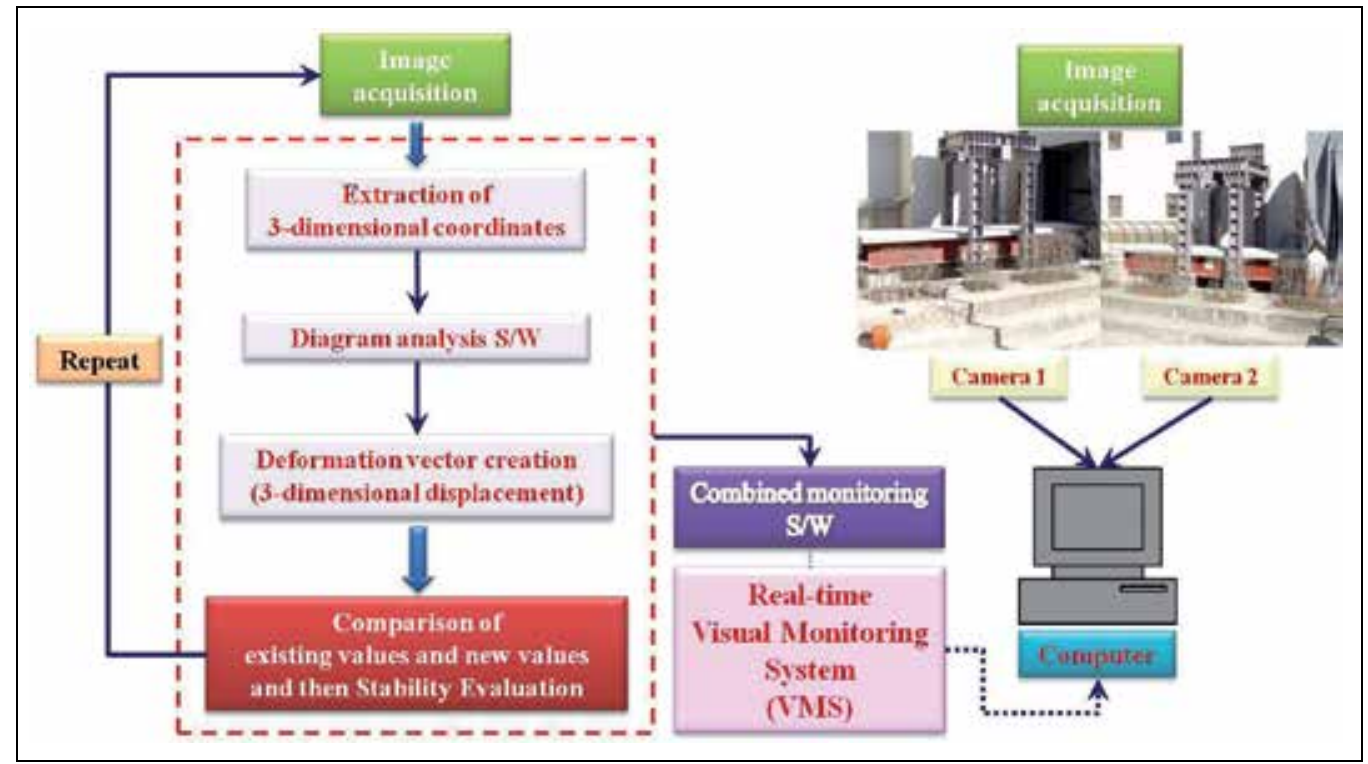

Fig. 1. Analysis procedure of VMS using digital photogrammetry

\subsection{Process of VMS}

The procedure of VMS is shown in Fig. 2. First of all, the image obtained from digital camera is conducted by image processing. Secondly, the modified image compares with the selected model image. Then it searches the model in the image. Finally, the measuring result is obtained by the searched result using image analysis module.

VMS is developed windows based program by Delphi and it consists of five tools as followed;

1. Project tool has camera information to photogrammetry

- Input focal length, pixel size and position (3-dimensinal coordinates) of cameras, and information of reference and unknown points

2. Second tool select analysis model

- Select size, range and area center of model for photogrammetry analysis, then modify the selected model using image processing wok

3. Third and fourth tools find metric image and model position in image, respectively

- Search the modified model of mertic image using geometric model finder

4. Fifth tool performs photogrammetry analysis and sees analysis results.

- Analyze measuring data using the combined photogrammetry analysis software 


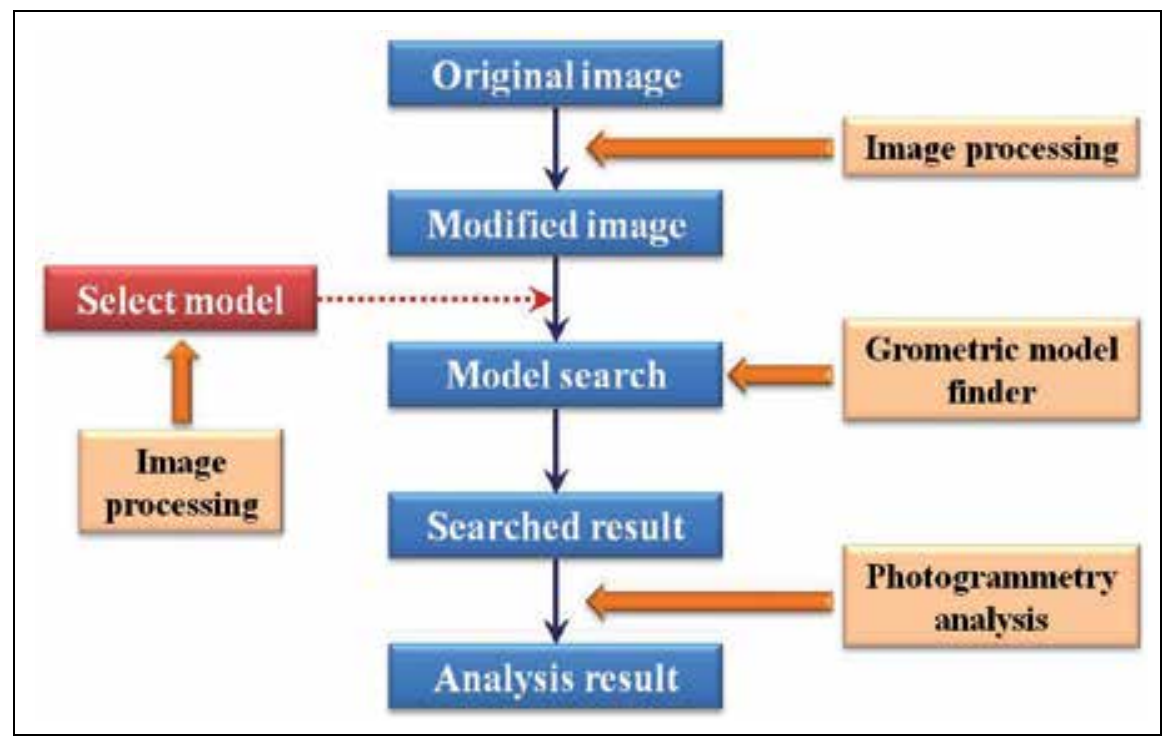

Fig. 2. Measuring analysis procedure of VMS

\subsubsection{Digital image processing}

Digital image processing has a procedure of threshold, histogram, contour extraction and image noise removal works. The image processing (in more detail, see Pratt, 1991) is summarized as followed;

1. Threshold

Image processing data is classified as colour image, gray image and binary image. Binary image can show an essential scene in the boundary value of black and white colour. That is why binary image is mostly applied to image processing even though it was not colour image. In general, colour image (original image, Fig 3(a)) can be converted to binary image, by applying threshold work as shown in Fig. 3(b).

2. Histogram and line extraction

Histogram is very important work to show the contrast information of the image. The histogram shows the distribution of the light and shade value in image. It simply presents an image pixel using bar graph as well.

Line extraction is to be defined as not only outline of object in the image, but also characteristic line of the image on image processing. The line extraction to present the characteristic of the image is important tool in common with histogram. Line extraction image is shown in Fig 3(c)

3. Image noise removal

If the image has noise, there is a clear difference between noise depth and its surroundings in the image. The noise removal using noise characteristic is defined as smoothing method. Also, the noise removal can improve image quality. 


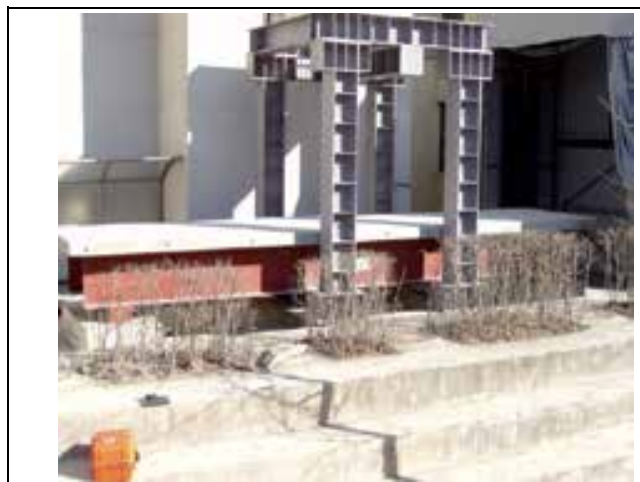

a)

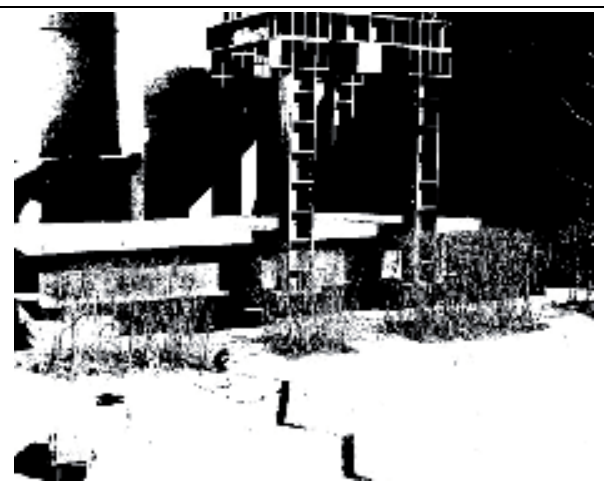

b)

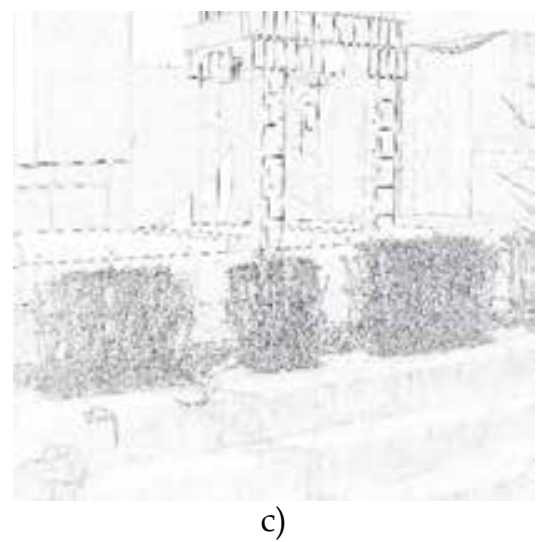

Fig. 3. Step of Digital image processing: a) original image, b) binary image applied in threshold; c) line extraction image

\subsubsection{Model finding}

The geometric model finder module is used in photogrammetry procedures because the model finding needs to search an unknown points corresponding to reference point. The function of geometric model finder module only extracts the required data from pattern recognition system based on the modified image by image processing. The characteristics of extracted data are distinguished by pre-processing. Then its pattern is recognized and processed by system. That is, VMS requires a pattern recognition procedure because it is automatic and complex system.

The procedure of pattern recognition is shown in Fig. 4. The metric image is pre-processed to pull out the required data because it includes unnecessary information. Next, the extracted data is divided into smaller units for facilitation of pattern recognition. Small units are idealized in both size and length. Finally, the characteristics are selected to obtain the important factors of pattern recognition, and then it is compared to the identification of patterns.

The pattern preprocessing includes preprocessing, partition and normalization. It is simple and does not require a lot of time in process. It does not change the required characteristics in pattern recognition. 
The characteristic extraction is the selecting process of the stabilized physical property for data transformation. Furthermore, the process includes both removal of redundancy property without loss of data information by reduction of pattern dimension and minimization of the processing time and storage space to pattern recognition. It means that the obtained data by measuring are converted into the required characteristics for pattern recognition. The methods of pattern matching, which is to identify the range of random pattern in the image, are theoretical analysis and structural analysis. Fig. 5 shows example in flow of pattern recognition system.

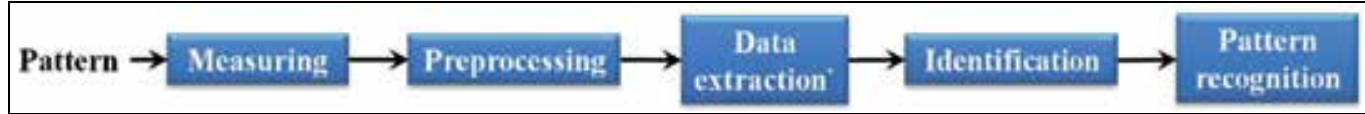

Fig. 4. Pattern recognition procedure

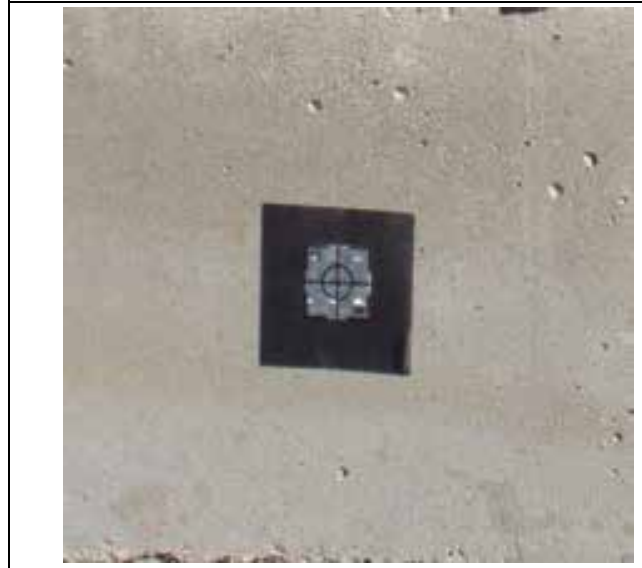

a)

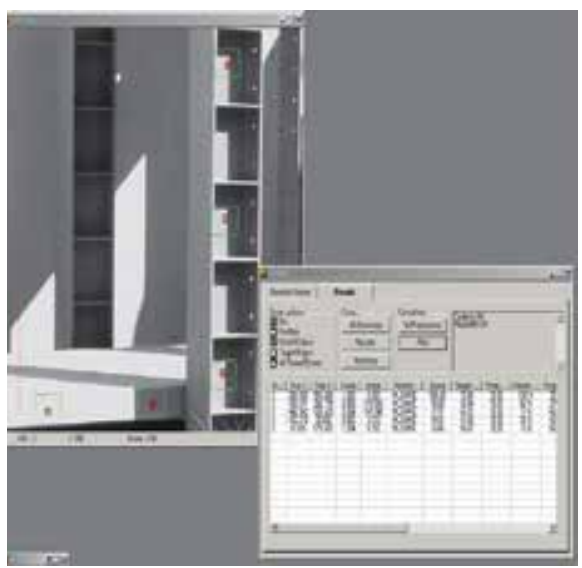

c)

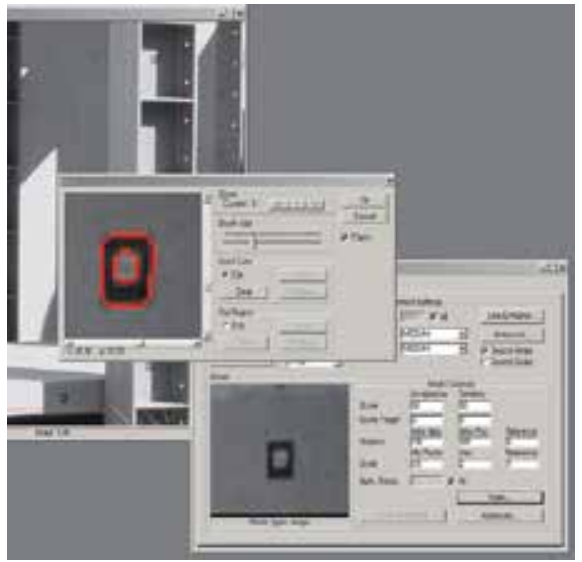

b)

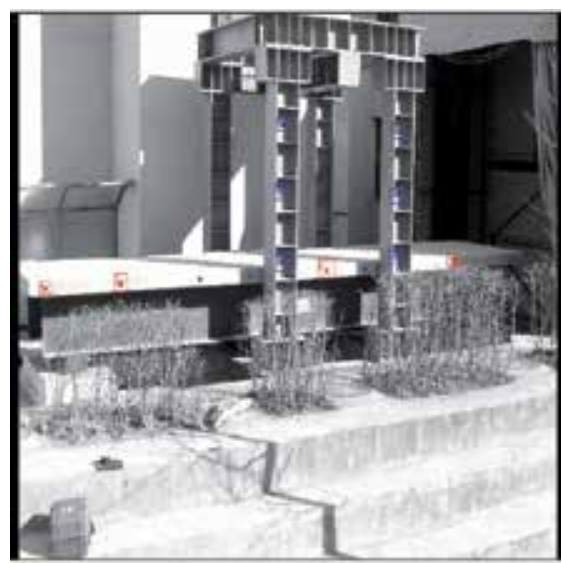

d)

Fig. 5. Flow of pattern recognition system: a) original model, b) preprocessing \& data extraction, c) identification \& pattern recognition, d) model search result (blue-reference point, red-unkown poins) 


\section{Experimental investigation using VMS}

Digital photogrammetry is applied in various fields. VMS has an important role for the deformation measuring of civil engineering structures as well as historic preservation and restoration of cultural assets.

The VMS results of tensile strength tests for welded members in civil structure, friction tests for concrete masonry block (CMU) retaining wall in civil engineering structure, and the measured deformations of cultural assets are presented.

\subsection{Deformation measuring of welded members}

The VMS and a test instrument (MTS-810) are used for the deformation measuring of the tensile strength tests of welded members. The accuracy and applicability was verified with comparing the deformation values of VMS to those of a test instrument. The characteristics of the behaviour in a welded joint were investigated by using VMS.

\subsubsection{Loading test}

In order to measure the local and overall deformations, measuring points are marked at a welded member as shown in both Fig. 6 and Fig. 7. U0 and L0 are measuring points to observe the overall deformation of the test member. U1 U10 and L1 L10 are measuring points to observe the local deformation near a welded joint at upper and lower part.

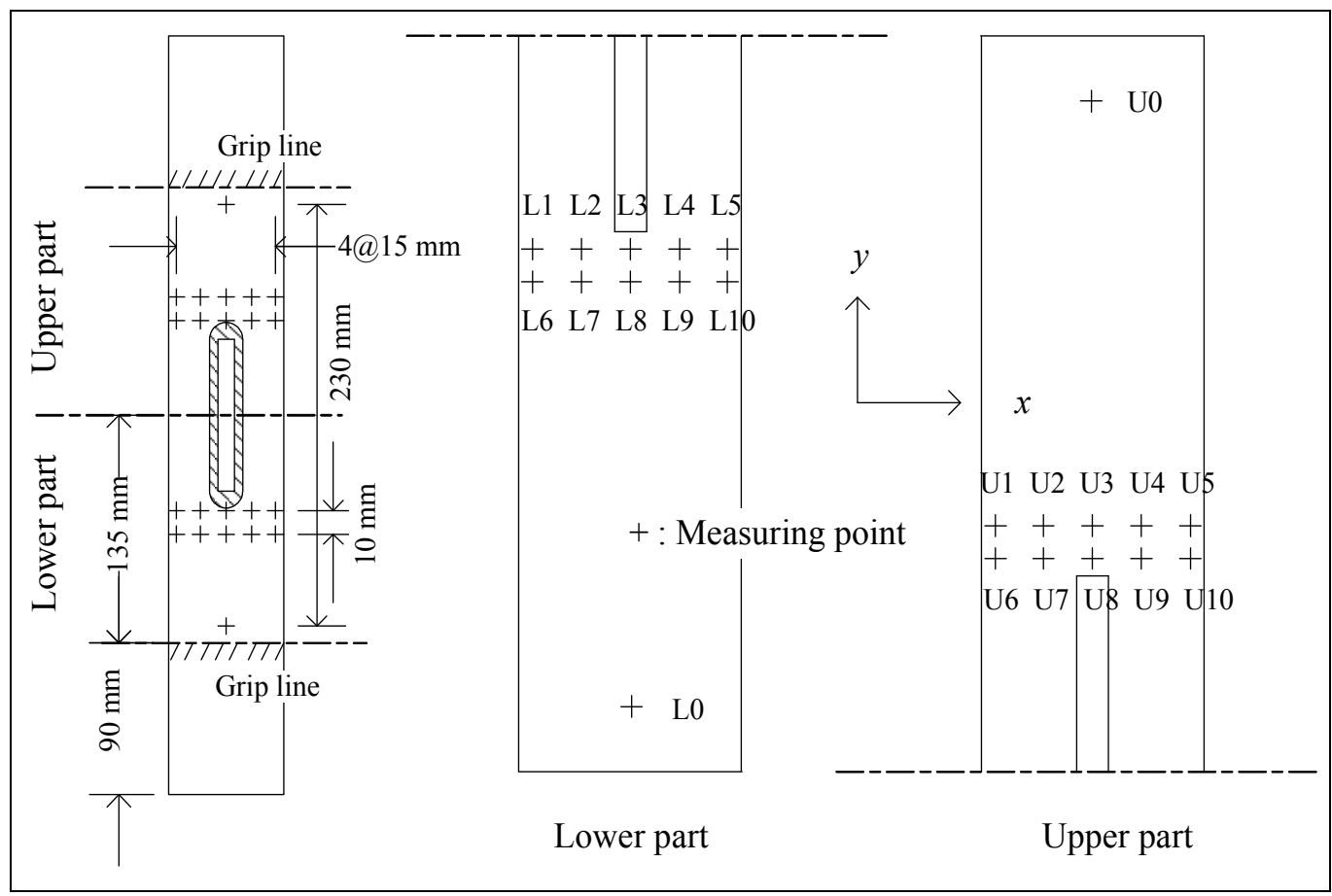

Fig. 6. Marked measuring points in welded member 


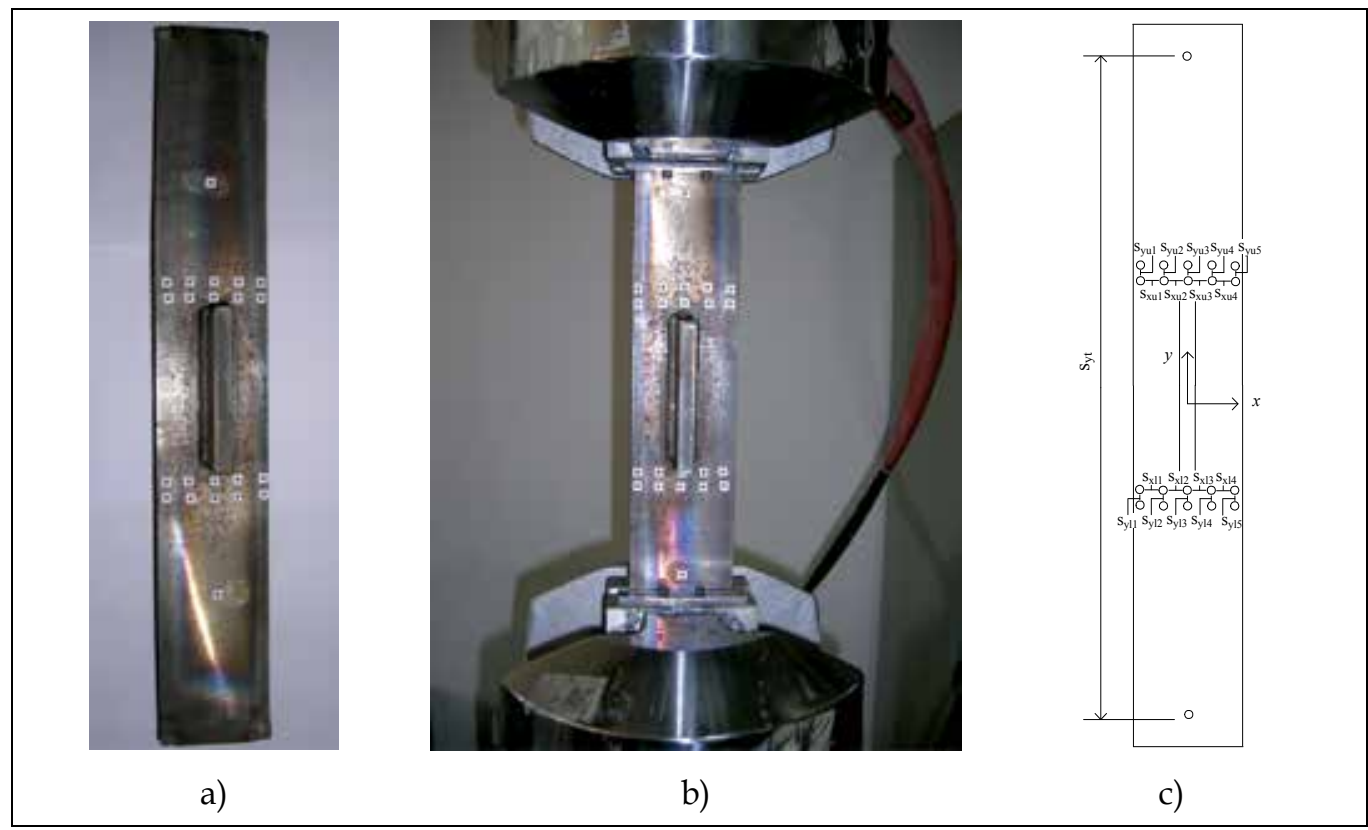

Fig. 7. View of welded member: a) welded member marked measuring points, $b$ ) tensile test rig, c) Strain models

To verify the accuracy and the applicability of VMS and investigate the characteristics of the deformation behaviour in a welded joint, strain values are calculated at each measuring points. Strains at each measuring point are shown in Fig. 8. Syt is VMS's overall strain values of a test member corresponding to those measured by a test instrument (MTS-810). Sxu1 Sxu10 and Syu1 Syu10 are strain values at upper part in the $x$-direction and the ydirection. Sxl1 Sxl10 and Syl1 Syl10 are strain values at lower part in the $\mathrm{x}$-direction and the $y$-direction.

The loading tests are conducted using MTS-810, which has loading capacity of $250 \mathrm{kN}$. The loading increasement is controlled by tensile displacement at a $0.01 \mathrm{~mm} / \mathrm{sec}$ with the assumption of static deformation. Also, the deformation is measured by two sets of digital camera, which are located at distance of $2.1 \mathrm{~m}$. VMS measures the deformation of measuring points at each 50 second. Fig. 7 shows a welded member marked measuring points and tensile test rig (Han et al., 2008).

\subsubsection{Comparison of results and analysis}

To confirm the accuracy and the applicability of VMS, strain (Syt) values of VMS are compared with those of a test instrument (MTS-810) as shown in Fig. 8. Syt values measured by VMS are good agreement with a stress-strain curve measured by MTS at the elastic and at the plastic regions. And it is seen that the developed VMS can accurately measure an overall deformation of a test member. It is concluded that the developed VMS has the high accuracy of $90 \%$ and the applicability of deformation measurement for the welded members. 


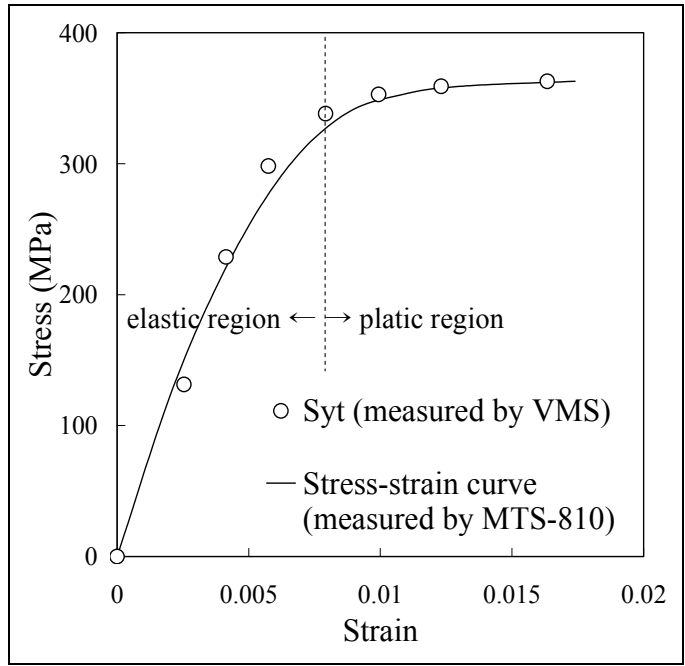

Fig. 8. Comparison of overall strains between VMS and MTS-810

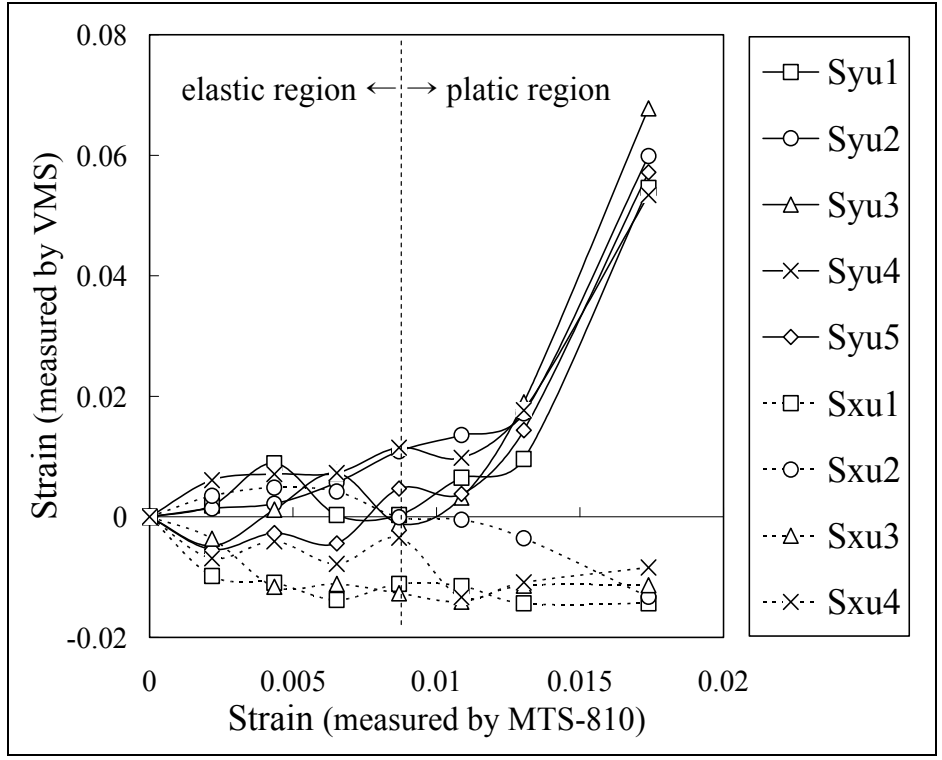

Fig. 9. Comparsion of strain distribution in a welded joint at upper part.

Strain values near a welded joint at upper part are shown Fig. 9. Strain values (Sxu1 Sxu4) in the $\mathrm{x}$-direction show compressive (-) up to -0.02 in the elastic region. In the plastic region, strain values constantly keep compressive. In the y-direction strain values (Syu1 Sxu5) are tensile $(+)$ about +0.01 in the elastic region. In the plastic region, strain values are rapidly larger up to about +0.06 .

It is shown from VMS measuring observations for a welded joint that strain values in the $x$ direction keep compressive up to -0.02. Strain values in the y-direction are tensile and rapidly increased up to +0.06 in the plastic region. 


\subsection{Displacement measuring of reinforced concrete masonry unit (CMU) retaining wall}

The manual measuring apparatuses like displacement transducer, settlement meter and total station are applied to perform both stability evaluation and maintenance of the reinforced CMU retaining wall. However, it is difficult to measure the overall deformation and crack of the reinforced CMU retaining wall because most manual measuring system can only measure the partial deformations. Also, the manual measuring systems have some disadvantages in durability and maintenance of apparatuses due to malfunctions. The malfunctions cause low accuracy for immediate reaction. Therefore, VMS is one of the good solutions for reasonable measuring of the reinforced CMU retaining wall because it can solve the disadvantages of the existing measuring systems. The forced displacement laboratory test of the reinforced CMU retaining wall is conducted to evaluate both the behaviour of reinforced CMU retaining wall and real-time measuring system at a long distance.

\subsubsection{Forced displacement laboratory test of reinforced $\mathrm{CMU}$ wall facing units}

Measuring both local displacement and overall deformation of CMU retaining wall facing units in real scale is performed. The test procedure is shown in Fig. 10 and follows as;

1. The rubber mats (like soft ground) is located to lead settlement of the wall facing units when vertical load is applied to the wall facing units

2. The wall facing units with three tiers is located on rubber mats of laboratory test apparatus

3. Obtain initial 3-dimensional coordinates of the image using VMS and total station

4. Obtain 3-dimensional coordinates from cameras using total station

5. Apply loads on the wall facing units

6. Obtain the deformed image coordinates of left and right due to the applied loads

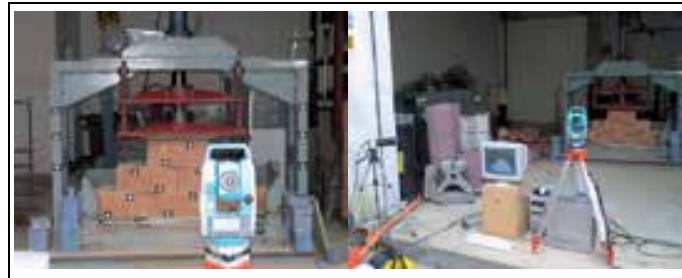

a)

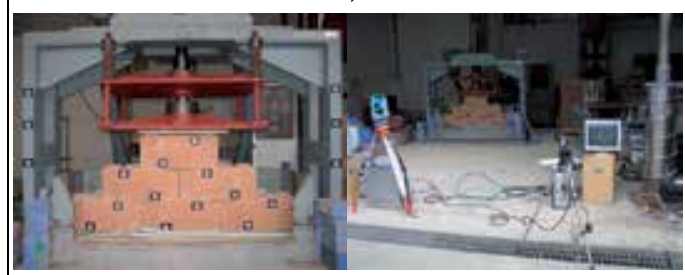

c)

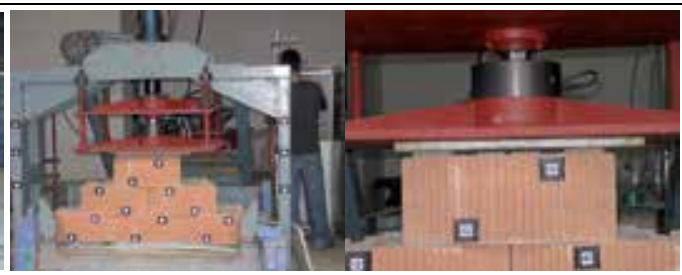

b)

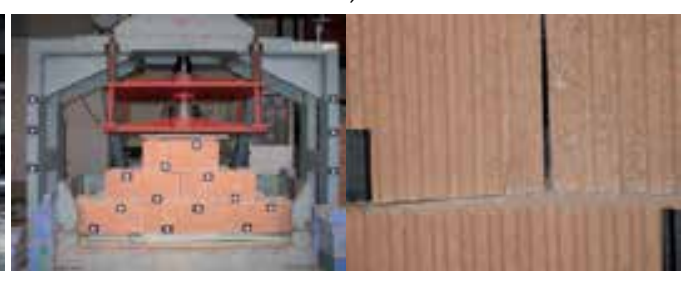

d)

Fig. 10. Test procedure: a) initial coordinates measuring (total station \& VMS), b) loading, c) displacement measuring, d) end of test 
The six tests are conducted. There are three measurments in vertical direction, two in horizontal measuring, and one simultanuous measuring in both vertical and horizontal directions. The test procedure is repeated at each case. The reference target points on steel frame of laboratory test apparatus and the unknown target points on the renforced CMU retaining wall face are located respectively as shown in Fig. 11. The movements of the target points are monitored by both total station and VMS (refer Fig. 12). The vertical loads apply from $30 \mathrm{kN}$ to $50 \mathrm{kN}$ and the vertical displacements are measured. The horizontal displacements occur due to the forced movement of rubber mats (Han et al., 2006).

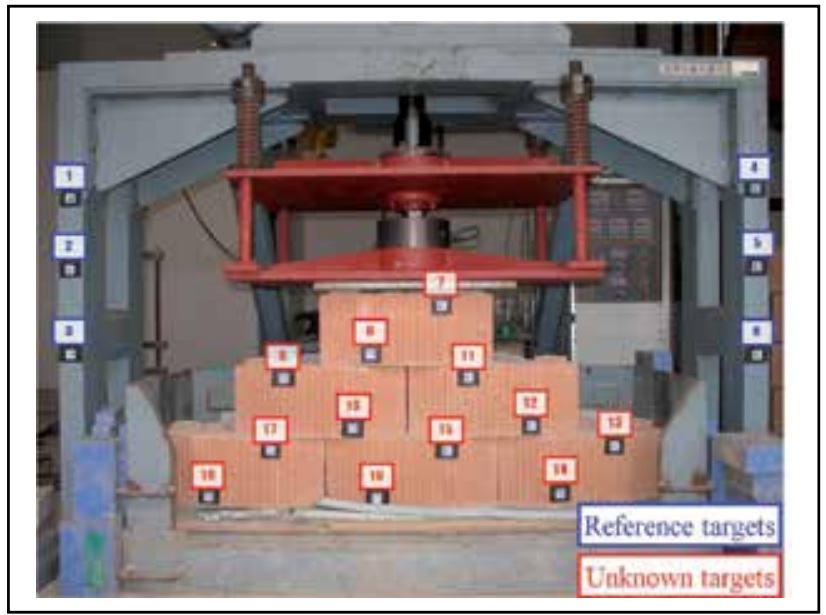

Fig. 11. Front view of the fixed targets on steel frame and CMU

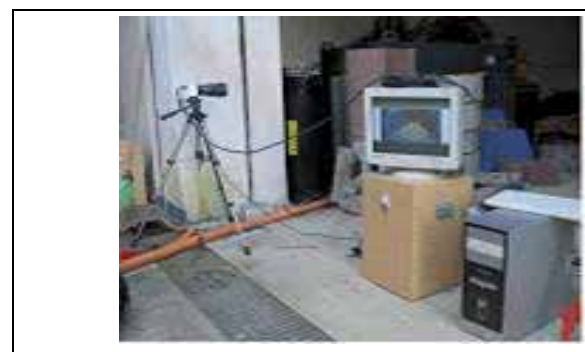

a)

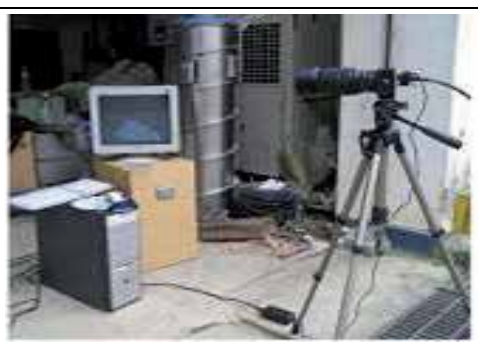

b)

Fig. 12. View of CCD cameras and computer system with VMS: a) left; b) right

\subsubsection{Comparison of results and analysis}

The initial coordinate for six reference target points and nine unknown target points is obtained by both VMS and total station. The displacements of wall facing units by vertical load and forced displacement are measured. The test results are shown in Table 1 . However, the measuring results of target points 13, 15 and 18 are eliminated because of dropping the target points off wall facing units during tests. The measuring difference between total station and VMS is from $0.1 \%$ to $16.7 \%$. Therefore, the measuring results of unknown points are only presented the maximum and minimum differences as shown in Table 1 . The average difference of total results is less than $3.6 \%$ as shown in Table 2 . 
The test results confirm the applicability of VMS as a good measuring system to the reinforced CMU retaining wall. Furthermore, the analysis results show that VMS can be real-time measuring systems while the targets are moved. Therefore, it is suggested that VMS can be a reasonable measuring method for reinforced CMU retaining wall systems.

\begin{tabular}{|c|c|c|c|c|c|c|c|c|c|c|}
\hline \multirow{2}{*}{$\begin{array}{c}\text { Un- } \\
\text { known } \\
\text { point }\end{array}$} & \multirow{2}{*}{$\begin{array}{c}\text { Test } \\
\text { classifi- } \\
\text { cation }\end{array}$} & \multicolumn{3}{|c|}{ Total station value } & \multicolumn{3}{|c|}{ VMS value } & \multirow{2}{*}{\begin{tabular}{|c|} 
Total \\
station \\
disp. \\
$(\mathrm{mm})$
\end{tabular}} & \multirow{2}{*}{$\begin{array}{l}\text { VMS } \\
\text { disp. } \\
(\mathrm{mm})\end{array}$} & \multirow{2}{*}{$\begin{array}{c}\text { Disp. } \\
\text { sub- } \\
\text { traction } \\
(\mathrm{mm})\end{array}$} \\
\hline & & $\mathrm{x}$ & $\mathrm{Y}$ & $\mathrm{z}$ & $\mathrm{x}^{\prime}$ & $y^{\prime}$ & $\mathrm{z}^{\prime}$ & & & \\
\hline \multirow{7}{*}{7} & $\begin{array}{l}\text { Ini. } \\
\text { coord. }\end{array}$ & 99563.742 & 99796.000 & -101887.196 & 99565.638 & 99796.911 & -101888.142 & & & \\
\hline & Case I & 99563.024 & 99785.000 & -101886.499 & 99565.948 & 99784.041 & -101887.468 & 11.045 & 12.891 & -1.846 \\
\hline & Case II & 99563.721 & 99780.000 & -101885.782 & 99566.378 & 99779.270 & -10188 & 16.062 & 17.657 & -1.594 \\
\hline & Case III & 99563.024 & 99774.000 & -101886.499 & 99565.159 & 99773.080 & -101887.266 & 22.023 & 23.852 & -1.829 \\
\hline & Case IV & 99584.871 & 99796. & -101 & 99587.203 & 99793.754 & -1018 & 21.954 & 22.587 & -0.633 \\
\hline & Case V & 99594.093 & 99794.000 & -101883 & 99596.535 & 99793.994 & -101 & 30.676 & 31.478 & -0.802 \\
\hline & Case VI & 99594.072 & 99778.000 & -101881.806 & 99597.074 & 99776.993 & & 35.679 & 37.526 & -1.847 \\
\hline \multicolumn{11}{|c|}{ Omission of measuring results (number $8,9,10,11,12,14$ and 17 ) } \\
\hline \multirow{7}{*}{16} & $\begin{array}{c}\text { Ini. } \\
\text { coord. }\end{array}$ & 99378.007 & 99266.000 & -101904.740 & 99377.803 & 99262.640 & -101904.771 & & & \\
\hline & Case I & 99377.987 & & & 99378.143 & 99250.941 & & 13.077 & 11.706 & 1.370 \\
\hline & Case II & 99377.987 & 99249.000 & -101903.326 & 99377.975 & 99246.205 & -101903.625 & 17.059 & 16.476 & 0.583 \\
\hline & Case III & 99378.704 & 99242.000 & -101904.023 & 99378.180 & 99238.361 & -101903.756 & 24.021 & 24.303 & -0.282 \\
\hline & Case IV & 99398.419 & 99266.000 & -101898.079 & 99398.882 & 99260.212 & -101898.850 & 21.471 & 22.029 & -0.558 \\
\hline & Case V & 99409.076 & 99263.000 & -101901.461 & 99408.924 & 99260.369 & -101901.005 & 31.385 & 31.430 & -0.045 \\
\hline & Case VI & 99409.055 & 99246.000 & -101900.047 & 99409.954 & 99242.347 & -101900.917 & 37.229 & 38.214 & -0.985 \\
\hline
\end{tabular}

* Case I: vertical load 30kN, Case II: vertical load 40kN, Case III: vertical load 50kN, Case IV: arbitrary horizontal displacement (1), Case V: arbitrary horizontal displacement(2), Case VI: vertical load and horizontal displacement

Table 1. Comparison between total station and VMS in measuring results

\begin{tabular}{|c|c|c|c|c|c|c|}
\hline \multirow{2}{*}{$\begin{array}{c}\text { Unknown } \\
\text { point }\end{array}$} & \multicolumn{7}{|c|}{ Test case } \\
\cline { 2 - 7 } & Case I & Case II & Case III & Case IV & Case V & Case VI \\
\hline 7 & 16.7 & 9.9 & 8.3 & 2.9 & 2.6 & 5.2 \\
\hline 8 & 8.9 & 3.3 & 4.4 & 1.3 & 0.5 & 3.9 \\
\hline 9 & 1.1 & 1.0 & 3.5 & 3.8 & 0.9 & 2.4 \\
\hline 10 & 2.7 & 2.4 & 2.0 & 6.7 & 2.3 & 2.3 \\
\hline 11 & 8.7 & 4.6 & 7.2 & 2.3 & 2.7 & 5.0 \\
\hline 12 & 1.2 & 1.3 & 3.6 & 3.9 & 2.4 & 3.8 \\
\hline 14 & 0.8 & 0.5 & 6.5 & 2.1 & 1.7 & 5.4 \\
\hline 16 & 10.5 & 3.4 & 1.2 & 2.6 & 0.1 & 2.6 \\
\hline 17 & 1.8 & 0.8 & 0.4 & 4.7 & 0.3 & 0.6 \\
\hline Average & $\mathbf{5 . 8}$ & $\mathbf{3 . 0}$ & $\mathbf{4 . 1}$ & $\mathbf{3 . 4}$ & $\mathbf{1 . 5}$ & $\mathbf{3 . 5}$ \\
\hline
\end{tabular}

Table 2. Measuring difference according to test cases (\%) 


\subsection{Field application of VMS to real structure}

\subsubsection{Simulated field test}

The field test of VMS is conducted for the concrete wall (refer Fig. 13), which is made of concrete, because of the limitation of field application on large structure. The simulation idea comes from large civil engineering structures made from concrete materials (Han et al., 2007). The field test is conducted to evaluate VMS application to concrete structure at long distance. The results of VMS compares with those of total station.

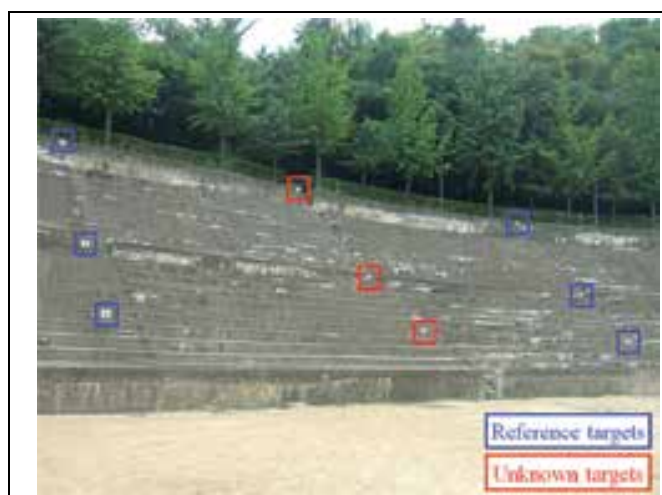

a)

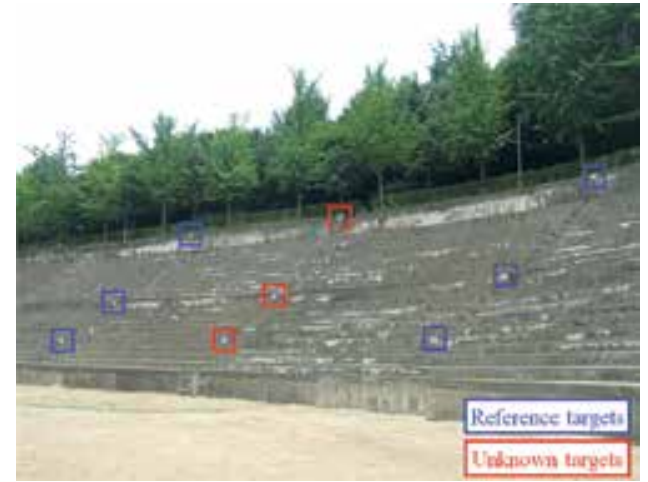

b)

Fig. 13. Simulation structure view for 3-dimensional image acquisition: a) left image, b) right image

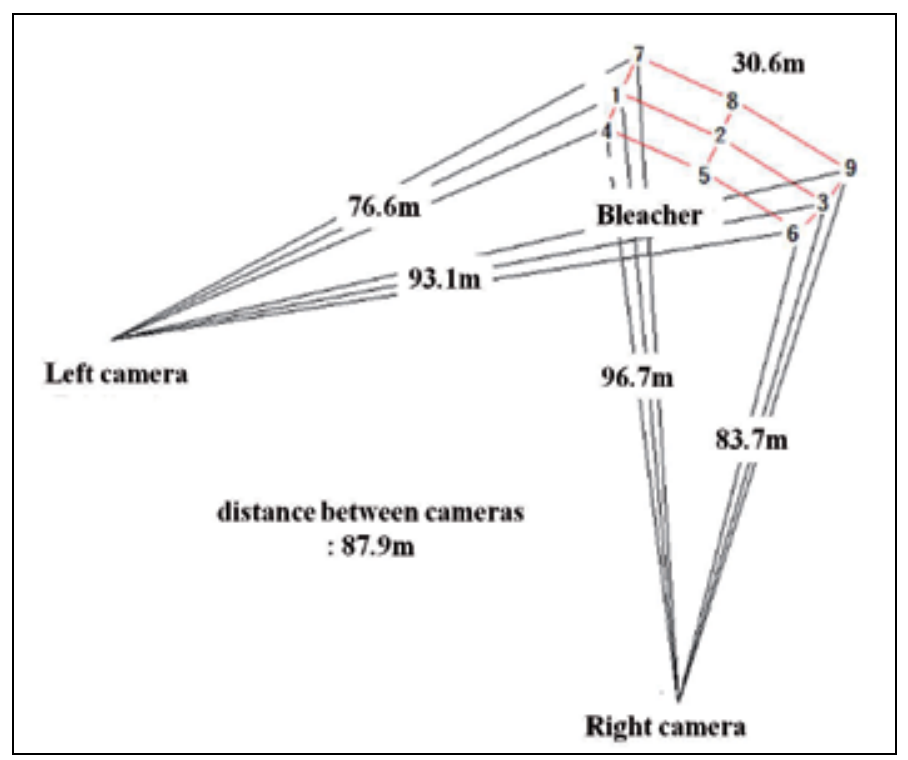

Fig. 14. Simulation schematic

The targets points are located on the concrete bleacher at the distance around $76 \mathrm{~m}$ to $97 \mathrm{~m}$ from CCD cameras and the distance between cameras is $87.9 \mathrm{~m}$ as show in Fig. 14. The 
reference target points (numbered as 1, 3, 4, 6, 7 and 9) and unknown target points (numbered as 2, 5 and 8) are located on the face of concrete bleacher, respectively (refer Fig. 13 and Fig. 14). The deformation of the targets points are measured by VMS system. As there was not a actual structural displacement for measuring, the unknown points have a simulated movement from initial position by the forced displacement as shown in Fig. 15. The forced displacement of targets points assumes $5 \mathrm{~cm}$ at top and bottom and $9 \mathrm{~cm}$ at left and right. Therefore, the each unknown point is measured in total of four steps. The simulation procedure is followed as;

1. Set up the CCD cameras and prepare VMS system (refer Fig. 16)

2. Fix reference point and unknown point targets points on object and then

3. Obtain initial 3-dimensional coordinates of the image using both VMS and total station

4. Obtain 3-dimensional coordinates of cameras using total station

5. Obtain images coordinates of left and right by forced displacement

6. Repeat (5) process according to the each step

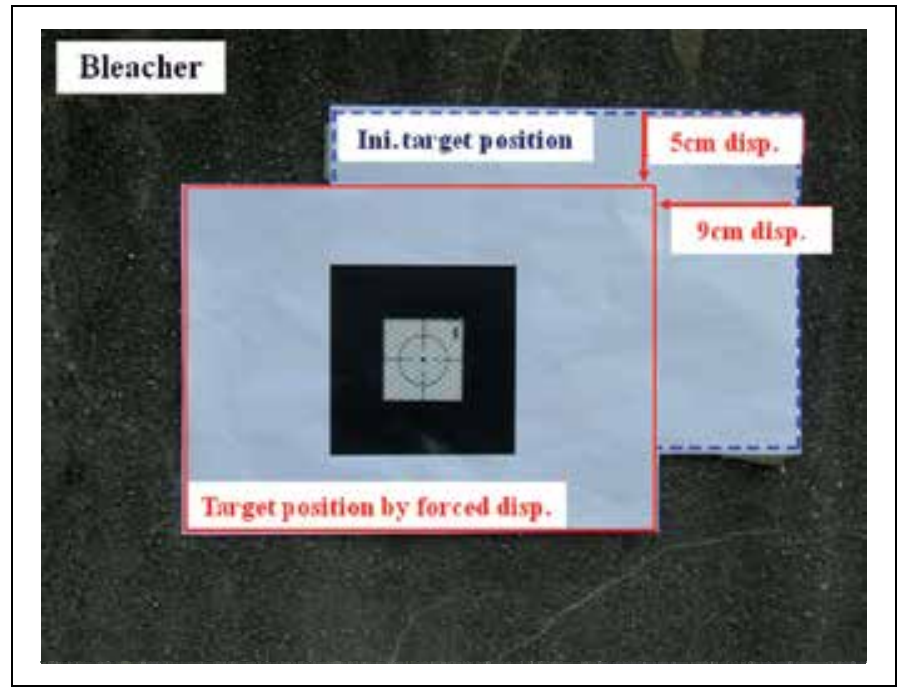

Fig. 15. Forced displacement of target

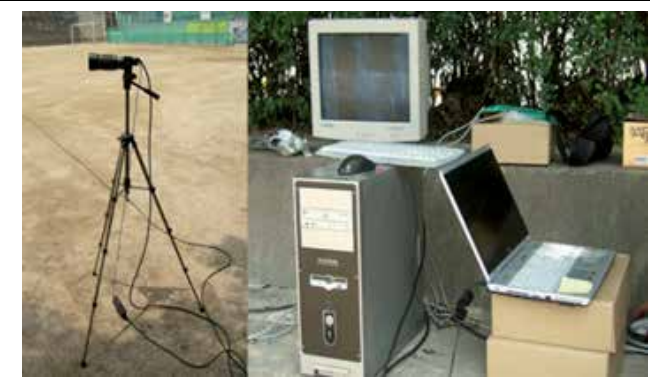

a)

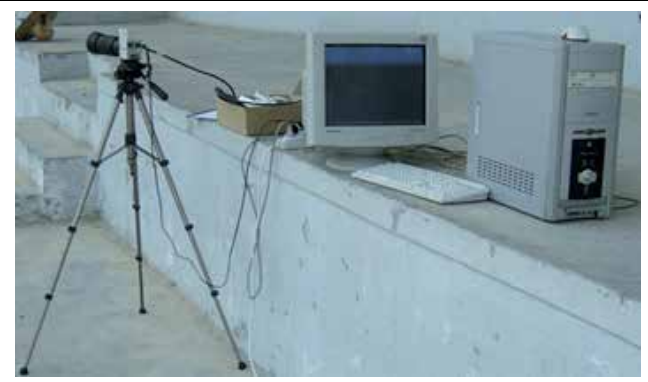

b)

Fig. 16. View of CCD cameras and computer system with VMS: a) left, b) right 


\subsubsection{Comparison of results and analysis}

The measuring results based on initial and forced displacement are analyzed as shown in Table 3 and 4 . The initial coordinates of the image using both total station and VMS are almost identical as shown in Table 3. The initial displacements of total station and VMS are zero. Also, the displacement according to the forced displacement is measured by both total station and VMS. The results are shown in Table 4. The comparison of measuring results shows that the difference is from $0.325 \mathrm{~mm}$ to $10.436 \mathrm{~mm}$ irregularly. However, the accuracy of VMS is acceptable because the calculated difference range based on camera pixel and measuring distance has maximum $10.436 \mathrm{~mm}$.

It means that VMS can apply to measure displacement, deformation and crack of civil engineering structures. In addition, the accuracy of VMS will be improved continuously because the error rate in camera capacity will be decreased by the industrial development related to image acquisition apparatuses.

\begin{tabular}{|c|c|c|c|c|c|c|c|c|}
\hline \multirow{2}{*}{$\begin{array}{l}\text { Targets } \\
\text { number }\end{array}$} & \multirow{2}{*}{$\begin{array}{l}\text { Test } \\
\text { step }\end{array}$} & \multicolumn{3}{|c|}{ Total station value } & \multicolumn{3}{|c|}{ VMS value } & \multirow{2}{*}{$\begin{array}{c}\text { Point } \\
\text { classification }\end{array}$} \\
\hline & & $\mathrm{x}$ & $\mathrm{y}$ & $\mathrm{z}$ & $x^{\prime}$ & $\mathrm{y}^{\prime}$ & $\mathrm{z}^{\prime}$ & \\
\hline 1 & \multirow{9}{*}{ Initial } & 37491 & 104055 & -170330 & 37491 & 104055 & -170330 & reference \\
\hline 2 & & 50732 & 104053 & -173430 & 50797 & 104052 & -173335 & Unknown \\
\hline 3 & & 66774 & 104083 & -173912 & 66774 & 104083 & -173912 & reference \\
\hline 4 & & 38404 & 101559 & -166586 & 38404 & 101559 & -166586 & reference \\
\hline 5 & & 51345 & 101437 & -169566 & 51398 & 101445 & -169534 & Unknown \\
\hline 6 & & 66096 & 101606 & -169967 & 66096 & 101606 & -169967 & reference \\
\hline 7 & & 36781 & 107416 & -173733 & 36781 & 107416 & -173733 & reference \\
\hline 8 & & 49892 & 107274 & -176846 & 49971 & 107247 & -176695 & Unknown \\
\hline 9 & & 66988 & 107393 & -177548 & 66988 & 107393 & -177548 & reference \\
\hline
\end{tabular}

Table 3. Initial coordinates of targets

\begin{tabular}{|c|c|c|c|c|c|c|c|c|c|c|}
\hline \multirow{2}{*}{$\begin{array}{l}\text { Test } \\
\text { step }\end{array}$} & \multirow{2}{*}{$\begin{array}{c}\text { Unknown } \\
\text { point }\end{array}$} & \multicolumn{3}{|c|}{ Total station value } & \multicolumn{3}{|c|}{ VMS value } & \multirow{2}{*}{$\begin{array}{l}\text { Total station } \\
\text { disp. } \\
\text { (mm) }\end{array}$} & \multirow{2}{*}{$\begin{array}{l}\text { VMS } \\
\text { disp. } \\
(\mathrm{mm})\end{array}$} & \multirow{2}{*}{$\begin{array}{c}\text { Disp. } \\
\text { subtraction } \\
(\mathrm{mm})\end{array}$} \\
\hline & & $\mathrm{x}$ & $\mathrm{y}$ & $\mathrm{z}$ & $x^{\prime}$ & $y^{\prime}$ & $\mathrm{z}^{\prime}$ & & & \\
\hline Step 1 & \multirow{4}{*}{5} & 51434 & 101386 & -169592 & 51486 & 101401 & $\mid-169558$ & 101.272 & 106.049 & -4.777 \\
\hline Step 2 & & 51431 & 101483 & -169592 & 51480 & 101498 & -169554 & 99.664 & 101.073 & -1.409 \\
\hline Step 3 & & 51255 & 101483 & -169545 & 51310 & 101497 & $\mid-169511$ & 104.771 & 102.619 & 2.153 \\
\hline Step 4 & & 51253 & 101392 & -169548 & 51309 & 101398 & -169518 & 101.912 & 104.530 & -2.619 \\
\hline Step 1 & & 50814 & 104014 & -173452 & 50888 & 104008 & $\mid-173358$ & 103.663 & 93.227 & 10.436 \\
\hline Step 2 & & 50816 & 104104 & -173448 & 50883 & 104100 & -173352 & 99.945 & 99.620 & 0.325 \\
\hline Step 3 & & 50641 & 104104 & -173408 & 50708 & 104098 & -173310 & 103.257 & 106.287 & -3.030 \\
\hline Step 4 & & 50636 & 104017 & -173415 & 50705 & 104008 & $\mid-173311$ & 104.766 & 103.350 & 1.416 \\
\hline Step 1 & & 49979 & 107232 & -176874 & 50060 & 107202 & -176724 & 103.860 & 100.538 & 3.322 \\
\hline Step 2 & & 49978 & 107321 & -176871 & 50050 & 107293 & $\mid-176719$ & 94.515 & 101.020 & -6.505 \\
\hline Step 3 & & 49796 & 107323 & -176833 & 49882 & 107290 & $\mid-176679$ & 100.130 & 108.735 & -8.605 \\
\hline Step 4 & & 49799 & 107228 & -176839 & 49884 & 107195 & -176682 & 102.186 & 104.239 & -2.052 \\
\hline
\end{tabular}

Table 4. Comparison results between Total station value and VMS value according to forced displacement 


\subsection{Case study for cultural assets}

\subsubsection{Overview}

Recently, there have been many attempts to restore cultural assets in South Korea. However, both maintenance and restoration of cultural assets are very difficult due to several factors like site conditions, natural deteriorations, damages and restoration methods. Therefore, the photogrammetry methods based on 3-dimensonal analysis was introduced as non-contact methods without damage of cultural assets (Kim et al., 2003).

The case study presents the application of a digital photogrammetry to seven-story stone tower in Sinsedong, Gyeongsangbuk-do, Korea for the maintenance data establishment of cultural assets as shown in Fig. 17 (Sin \& Kim, 2006; Yoon, 2006).

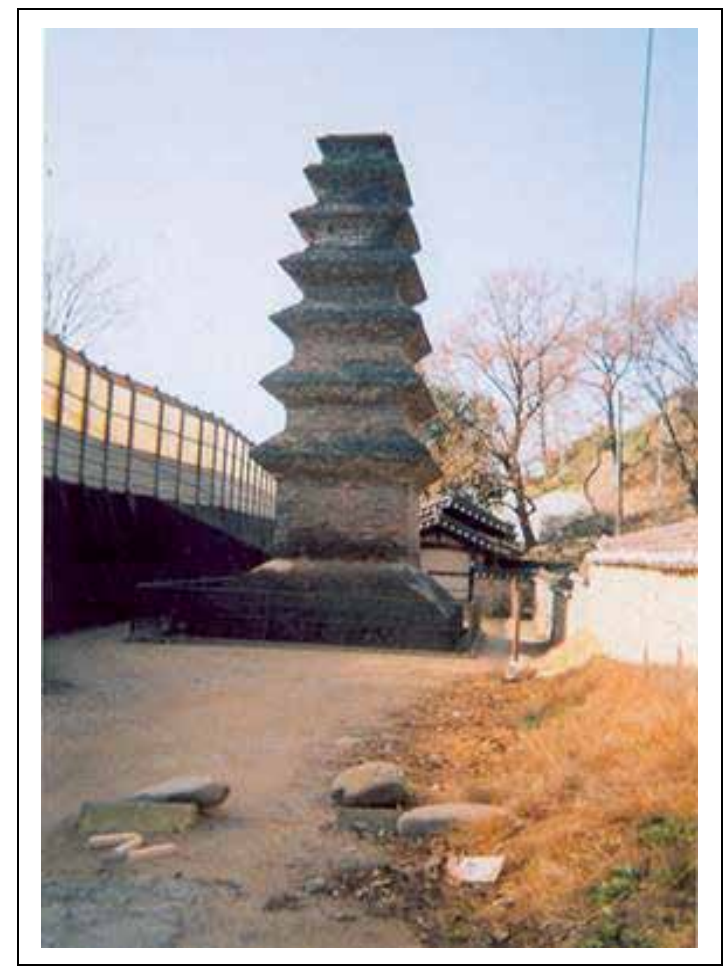

Fig. 17. Seven-story stone tower in Sinsedong, Gyeongsangbuk-do, Korea (Sin \& Kim, 2006; Yoon, 2006)

\subsubsection{Application result and analysis}

The targets are fixed considering obstacles around seven-story stone tower in Sinsedong, Gyeongsangbuk-do, Korea. The front and back images at the top, the middle and the bottom positions are measured by CCD cameras as shown in Fig. 18. 3-dimensional coordinates are obtained by the photogrammetry process based on acquired images and decided by RolleiMetric CDW (Close-range digital workstation). The drawing of seven-story stone tower is presented as shown in Fig. 19. 


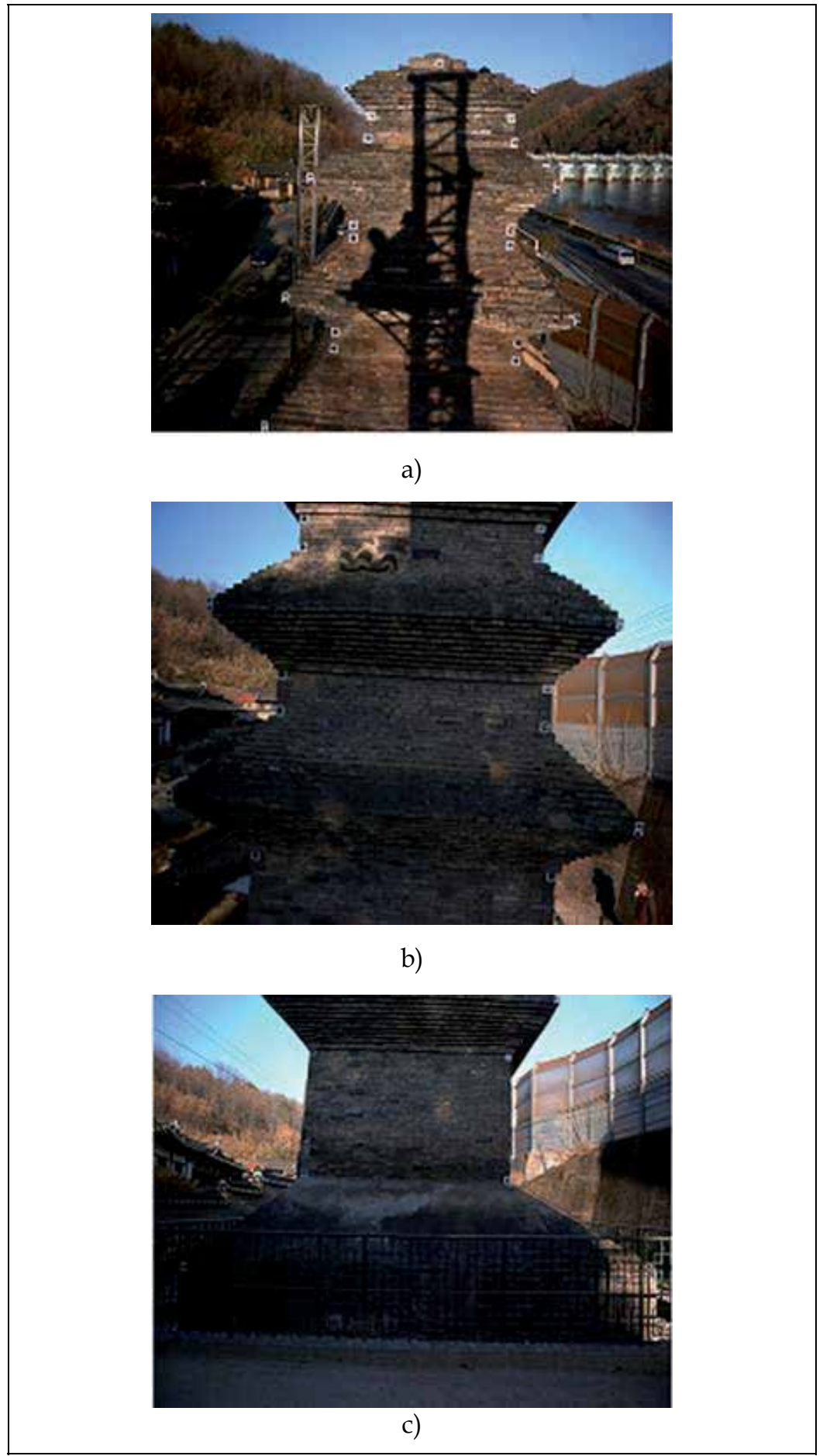

Fig. 18. Targets image acquisition according to each position: a) top, b) middle, c) bottom (Sin \& Kim, 2006; Yoon, 2006) 


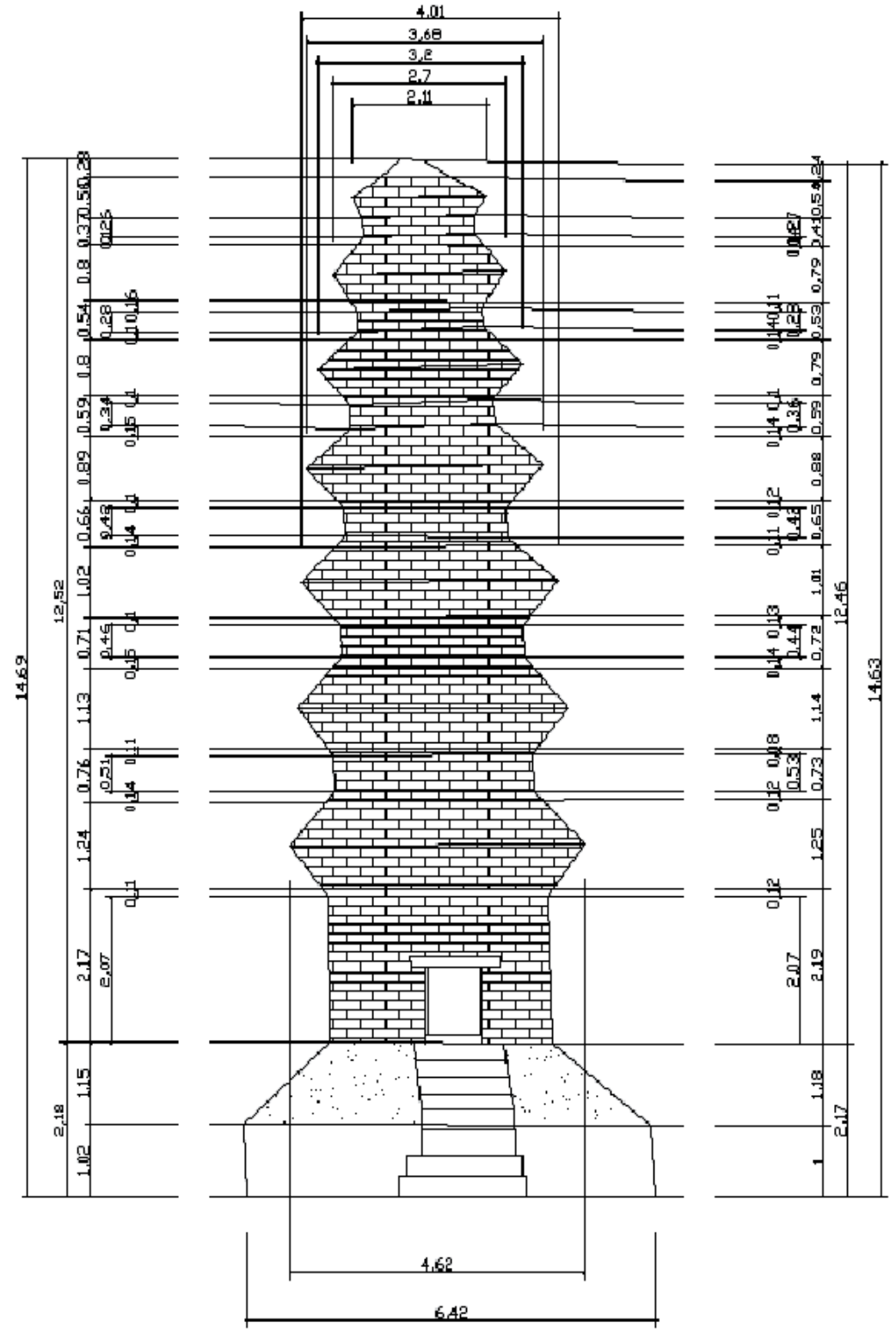

a) 


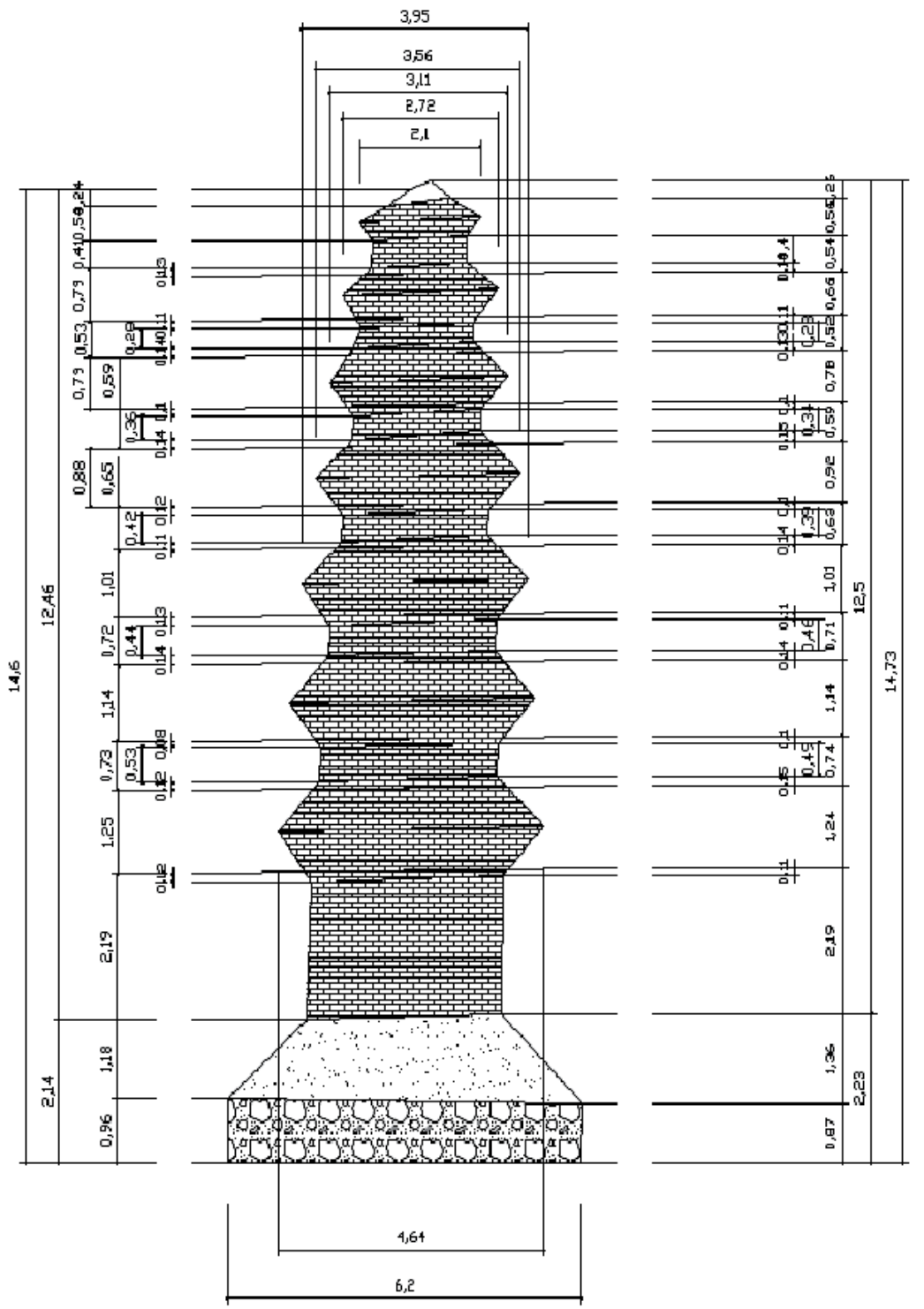

b)

Fig. 19. Drawing results of stone tower: a) front, b) back (Sin \& Kim, 2006; Yoon, 2006)

The stability of the seven-story stone tower is evaluated by the results of photogrammetry analysis. The stability evaluation is as followed;

1. Extend and determine to vertical axis for center of the seven-story stone tower

2. Evaluate incline of the seven-story stone tower based on the determined vertical axis 
The evaluation results are shown in Fig. 20. In front view, the incline and the maximum eccentricity are $1.3^{\circ}$ and $0.336 \mathrm{~m}$ on the left, respectively. In back view, the incline and the maximum eccentricity are $0.78^{\circ}$ and $0.251 \mathrm{~m}$ on the right, respectively. Therefore, the digital image information based on the results of photogrammetry analysis can be applied to space analysis like cross section and inclination. It means that digital photogrammetry can be used for stability evaluation, maintenance and restoration of cultural assets.

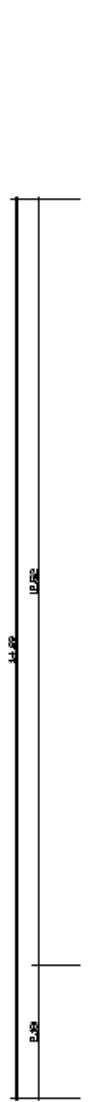

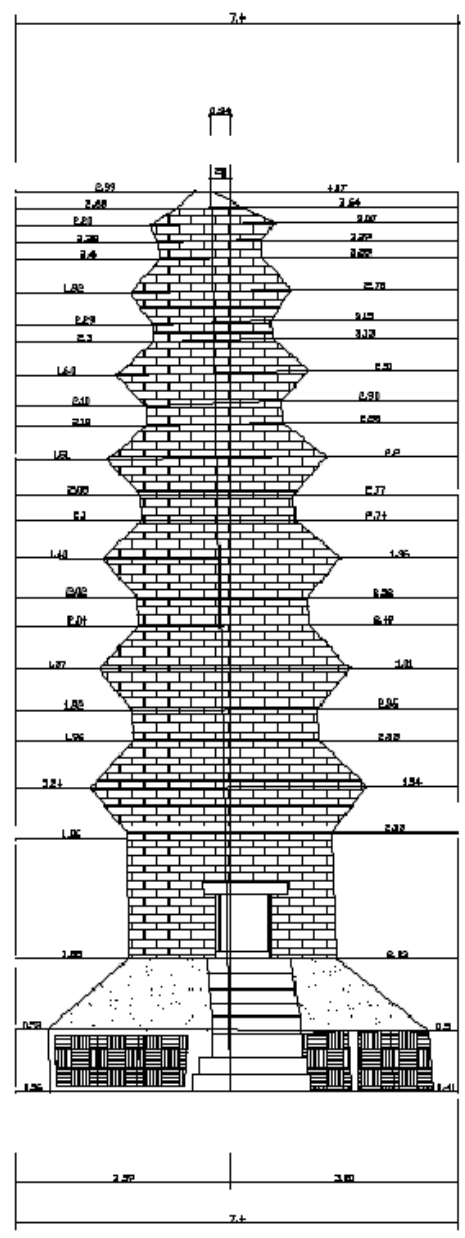

a)

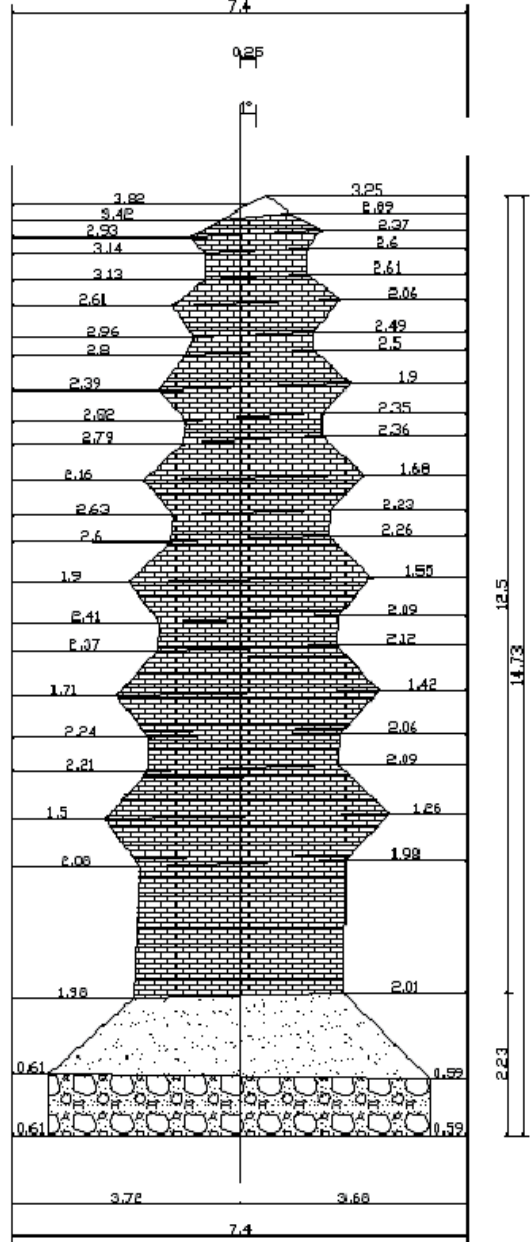

b)

Fig. 20. Incline analysis results: a) front, b) back (Sin \& Kim, 2006; Yoon, 2006)

\section{Conclusion}

This chapter introduces research cases related to photogrammetry and a real-time measuring system (called as visual monitoring system, VMS) which is based on digital photogrammetry. It confirms the applicability of digital photogrammetry in civil engineering structures and cultural assets. The research conclusions are followed as; 
1. In deformation measuring of welded members, the accuracy and applicability of VMS was verified by comparing the deformation values of VMS with those of the loading test instrument (MTS-810). It is seen that the developed VMS has the high precision comparison with test results using MTS-810.

2. VMS is applied to displacement measuring of reinforced CMU retaining wall. The average difference of VMS is less than $3.6 \%$ in total test cases. This means that the measuring result was verified reasonably in all target points except the dropped target.

3. The accuracy of VMS based on simulated field test is satisfied in theoritical difference range. Therefore, the its applicability was verified reasonably in civil engineering structure

4. It introduces the applicability of digital photogrammetry in maintenance and restoration of cultural assets. The result confirmed that the stability of cultural assets is evaluated by digital photogrammetry. Also, it can be applied as maintenance technique.

Digital photogrammetry is applied in various fields and it is developed rapidly with development of image processing techniqe and image acquisition apparatus. Therefore, if digital photogrammetry is applied continuously in civil engineering structures, micro measuring instruments, and cultural assets, it is a very useful tool as disaster prevention monitoring system due to the quantitative digital image. However, image acquisition apparatus should be improved in high precision. Techniques of camera capacity should be developed to decrease an error rate of image acquisition apparatus. With high techniques, real-time monitoring system will be used more widely in various fields.

\section{References}

Bae, S. H. (2000). The component development of digital photogrammetry for the construction structure displacement analysis, Doctoral thesis, Chungnam national university, Korea (in Korean)

El-Hakim, S. F. \& Wong, K. W. (1990). Working group V/1: digital and real-time close-range photogrammetry, Close-Range Photogrammetry Meets Machine Vision, SPIE Proceedings, Vol. 1395, p. 2, Bellingham, Washington, USA, 1990

Fraser, C. S. (1993). A resume of some industrial applications of photogrammetry, ISPRS Journal of Photogrammetry and Remote Sensing, Vol.48, No.3, pp. 12-23, ISSN 09242716

Fraser, C. S. (1997). Full Automation of Sensor Calibration, Exterior Orientation and Triangulation in Industrial Vision Metrology, International Workshop on Image analysis and information fusion, pp. 13-24, ISBN 0646330691, Adelaide, Australia, November, 1997

Gruen A. (1992). Recent advances of photogrammetry in robot vision, ISPRS Journal of Photogrammetry and Remote Sensing, Vol. 47, No. 4, pp. 307-323, ISSN 0924-2716

Han, J. G.; Bae, S. H. \& Oh, D. Y. (2001). Application of photogrammetry method to measurement ground-surface displacement on the slope, J. Korean Env. Res \& Reveg. Tech, Vol.4, no.3, pp. 10-18, ISSN 1229-3032 (in Korean)

Han, J. G.; Chang, K. H.; Jang, G. C.; Hong, K. K.; Cho, S. D.; Kim, Y. S.; Kim, J. M. \& Shin, Y. E. (2008). Development of a Visual Monitoring System for Deformation Measuring of Welded Members and Its Application, Materials Science Forum, Vols. 580-582, pp. 557-560, ISSN 1662-9752 
Han, J. G.; Hong, K. K.; Kwak, K. S. \& Cho, S. D. (2005). Simulation Evaluation of Visual Monitoring System for The Dam Measuring, Proceedings of KSCE 2005 Conference, pp. 3982-3985, Jeju Special Self-Governing Province, Korea, October 20-21, 2005 (in Korean)

Han, J. G.; Hong, K. K.; Kim, Y. S.; Cho, S. D. \& Kwak, K. S. (2007). Development of Automatic Displacement Measuring System using 3D Digital Photogrammetry Image and Its Application, Journal of the Korean Geotechnical Society, Vol.23, No.5, pp. 1-10, ISSN 1229-2427 (in Korean)

Han, J. G.; Jeong, Y. W.; Hong, K. K.; Cho, S. D.; Kim, Y. S. \& Bae, S. H. (2006). Displacement Measuring Lab. Test of Reinforced-Soil Retaining Wall Block using 3D Digital Photogrammetry Image, Journal of the Korean Geosynthetics Society, Vol. 5, No. 3, pp. 45-52, ISSN 1975-2423 (in Korean)

Han, J. G. \& Song, Y. S. (2003). Displacement Measuring System for the Slope Stability Analysis Using the Softcopy Photogrammetry, Journal of the Korean Geotechnical Society, Vol.19, no.4, pp. 23-32, ISSN 1229-2427 (in Korean)

Hannah, M. J. (1989). A system for digital stereo image matching, Photogrammatic Engineering and Remote Sensing, Vol.55, No.12, pp. 1765-1770, ISSN 0099-1112

Kang, J. M.; Yoon, H. C. \& Bae, S. H. (1995). A Study on the 3-D Deformation Analysis for Safety Diagnosis of Bridges, Journal of the Korean Society of Surveying, Geodesy, Photogrammetry, and Cartography, Vol.13, No.2, pp. 69-76, ISSN 1598-4850 (in Korean)

Kim, J. S.; Park, W. Y. \& Hong, S. H. (2003). The 3D Modelling of Cultural Heritage Using Digital Photogrammetry, Journal of the Korean Society of Surveying, Geodesy, Photogrammetry, and Cartography, Vol. 21, No. 4, pp. 365-371, ISSN 1598-4850 (in Korean)

Kraus, K. (1993). Photogrammetry, Vol. 1, Dümmler Verlag, Bonn, Germany

Kraus, K. (1997). Photogrammetry, Vol. 2, Dümmler Verlag, Bonn, Germany

Kraus, K. (2007). Photogrammetry: Geometry from images and laser scans, Walter de Gruyter, ISBN 978-3-11-019007-6, Berlin, Germany

Lee, S. C.; Hwang E. J. \& Han J. G. (2006). Efficient Image Retrieval Based on Minimal Spatial Relationships, Journal of Information Science and Engineering, Vol.22, No.2, pp. 447-459, ISSN 1016-2364

Lichti, D. D. \& Chapman, M. A. (1995). CCD camera calibration using the finite element method, Progress in Biomedical Optics and Imaging(Videometrics IV;SPIE Proceedings Series), No. 2598, pp. 34-43, ISSN 1605-7422, Philadelphia, USA, October, 1995

Munjy, R. A. H. (1985). A finite element analysis of the effect of having fiducial marks in non-topographic photogrammetry, Annual Meeting 51st, pp. 160-169, Washington, USA, March 10-15, 1985

Munjy, R. A. H. (1986). Calibrating non-metric cameras using the finite-element method, Photogrammetric engineering and remote sensing, Vol.52, No.8, pp. 1201-1206, ISSN 0099-1112

Ohnishi, Y.; Nishiyama, S.; Yano, T.; Matsuyama, H. \& Amano, K. (2006). A study of the application of digital photogrammetry to slope monitoring systems, International Journal of Rock Mechanics E Mining Sciences, Vol.43, No.5, pp. 756-766, ISSN: 13651609 
Park, W. Y.; Kim, J. S. \& Lee, I. S. (2001). Monitoring of Unsafe Dam Using GPS, Journal of the Korean Society of Civil Engineers, Vol.21, No.3-D, pp. 383-392, ISSN 1015-6348 (in Korean)

Pratt, W. K. (1991). Digital Image Processing, Wiley, New York, USA

Shortis, M. R.; Clarke, T. A. \& Short, T. (1994). Comparison of some techniques for the subpixel location of discrete target images, Progress in Biomedical Optics and Imaging(Videometrics III;SPIE Proceedings Series), No. 2350, pp. 239-250, ISSN 16057422, Boston, USA, November, 1994

Sin, D. Y. \& Kim, J. S. (2006). Analysis of cultural assets with digital photogrammetry, Journal of the Korean Society of Cadastre, Vol. 22, No. 2, pp. 91-99. ISSN 1226-9662

Shortis, M. R. \& Fraser, C. S. (1998). State of the Art of 3D Measurment Systems for Industrial and Engineering Applications, 21st International Congress of FIG, Brighton, U.K., July 19-25, 1998

Stewart, M. \& Tsakiri, M. (2001). Long-term dam surface monitoring using the global positioning system, Electronic Journal of Geotechnical Engineering, Vol.6, ISSN 10893032, Available from http://www.ejge.com/2001/Ppr0118/Abs0118.htm

Yoon, S. B. (2006), (A) Study on the Analysis and Restoration of Cultural Assets With Digital Photogrammetry, Master's thesis, Sangju university, Korea (in Korean) 


\title{
Photogrammetry for Archaeological Documentation and Cultural Heritage Conservation
}

\author{
Rami AL-Ruzouq \\ Al-Balqa' Applied University \\ Jordan
}

\section{Introduction}

Photogrammetry is the art and science of deriving accurate 3-D metric and descriptive object information from multiple analogue and digital images. Recently, there has been an increasing interest in utilizing imagery in different fields such as archaeology, architecture, mechanical inspection, and surgery. Photogrammetry is concerned with deriving measurements of the size, shape, position and texture of objects from measurements made on photographs. In its simplest form, a pair of overlapping photographs is used to create a three-dimensional model, with the use of appropriate instrumentation can yield quantifiable dimensions of the object. Traditionally, these dimensions were represented on maps and plans, either as elevations, facades and/or contours. The use of photogrammetry as a tool to aid in the documentation of cultural heritage has a long history, and is well established as a measurement science. Recent advances in the science make the techniques much more flexible in their application and present new opportunities in the representation of monuments as diverse as aboriginal rock painting shelters, historically significant buildings and structures, and culturally significant precincts or districts.

The archaeological heritage constitutes the basic record of past human activities. Its protection and proper management is therefore essential to enable archaeologists and other scholars to study and interpret it for the benefit of present and future generations. In this chapter, Geomatics techniques will be used for archaeological documentation of one of the most important sites in Jordan (Aqaba Fort). The city of Aqaba, Jordan is located along the

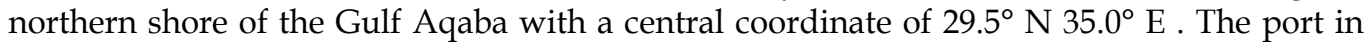
Aqaba provides the country of Jordan with their only marine site for the import and export of goods. It is also considered as one of the most popular tourist resort in Jordan and the Middle East where warm climate, beaches, and coral reefs. Aqaba is connected to the urban centres of Jordan to the north by two highway. There are overland border points to Eilat (5 $\mathrm{km}$ north) and Saudi Arabia (16 km south), and ferries connecting Jordan directly to Egypt. The 'Aqaba' is short of the old Arabic naming Aqaba Ayla, meaning 'Pass of Ayla'.

GIS will be used to create vector layers of the main architecture component of the fort. Photogrammetry will be employed to generate a digital elevation model and an Orthophoto which can be used together to give an actual terrain view for the historical structure 
while close range photogrammetry would show the three dimensional building with real coordinates and texture. As a result, stand alone application for one of the most popular archaeological sites in Jordan .Graphic user interface consist of three main items; Geographic Information System that include location using GPS, Landuse, Ortho-photo, Geology, Digital elevation Model and three dimensional model for the Aqaba fort using close range photogrammetry.

\section{Historical background}

The city of Aqaba, Jordan is located along the northern shore of the Gulf Aqaba with a central coordinate of $29.5^{\circ} \mathrm{N} 35.0^{\circ}$ E, Figure 1. Aqaba established a special economic zone with reduced taxation. The port of Aqaba provides Jordan the only marine site for the import and export of good.

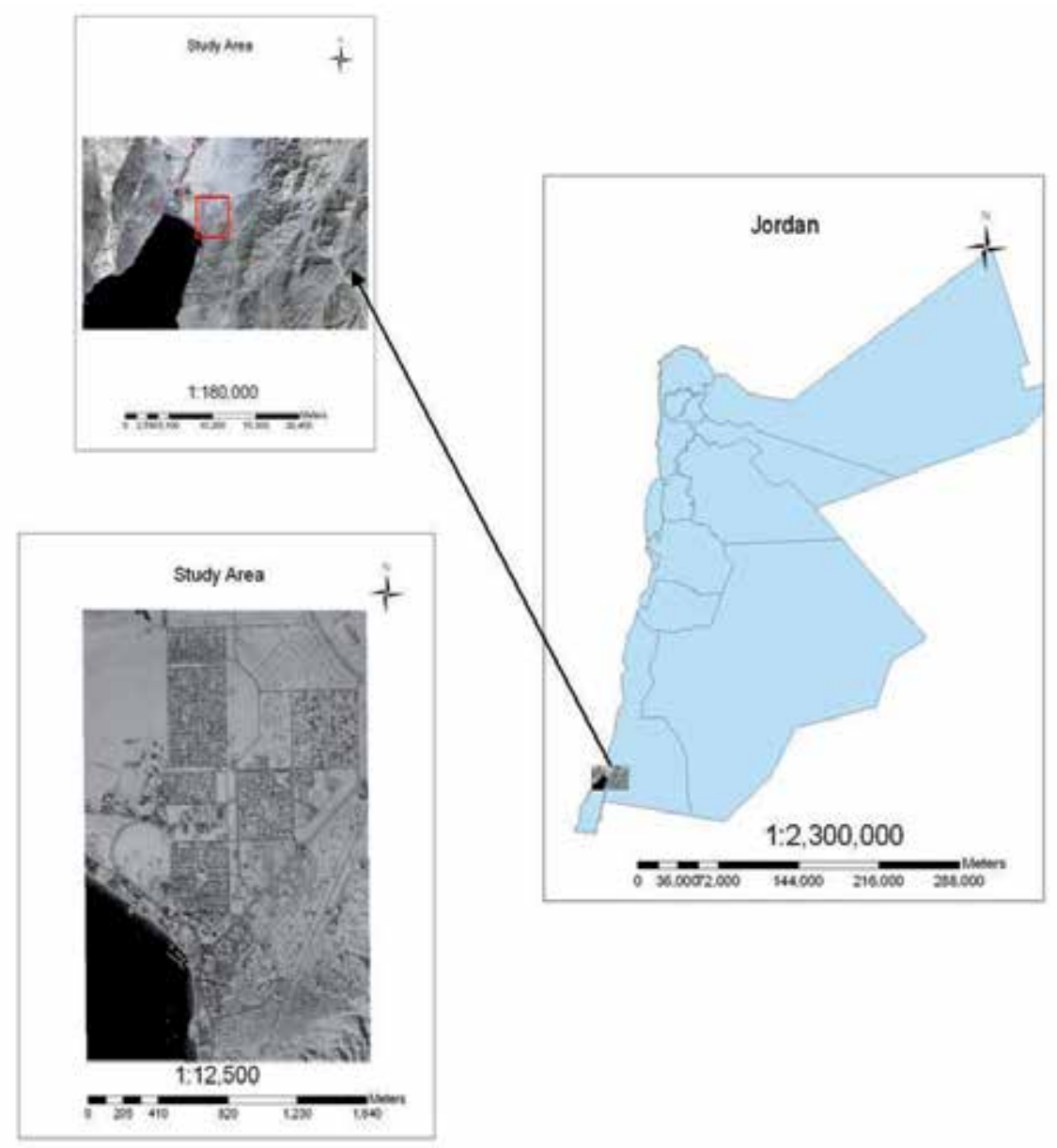

Fig. 1. Aerial photo of the study area.

Aqaba' is the short of the old Arabic naming Aqaba Ayla, meaning 'Pass of Ayla'. Aqaba is a coastal town with a population of 101,290 at year 2000 . 
The reason for building a fort on the shore was probably from the 13th century onwards connected with the involvement of the Mamluk sultans, in protecting the Egyptian pilgrimage to Holy cities of Arabia, When Ayla served as a stopping point for caravans. The castle , Figure 2 has the form of rectangle enclosure $(56.5 \mathrm{~m}$ by $58 \mathrm{~m})$. With towers projecting form the corners and a twin towered gatehouse. Ranged around the inside faces of the walls were rows of individual cells, arranged at two levels and evidently intended to provide accommodation for pilgrims. On the South wall stood a mosque, of which only the qibla wall and mihrab now survive. Aerial view of the Aqaba fort appear in Figure 3.

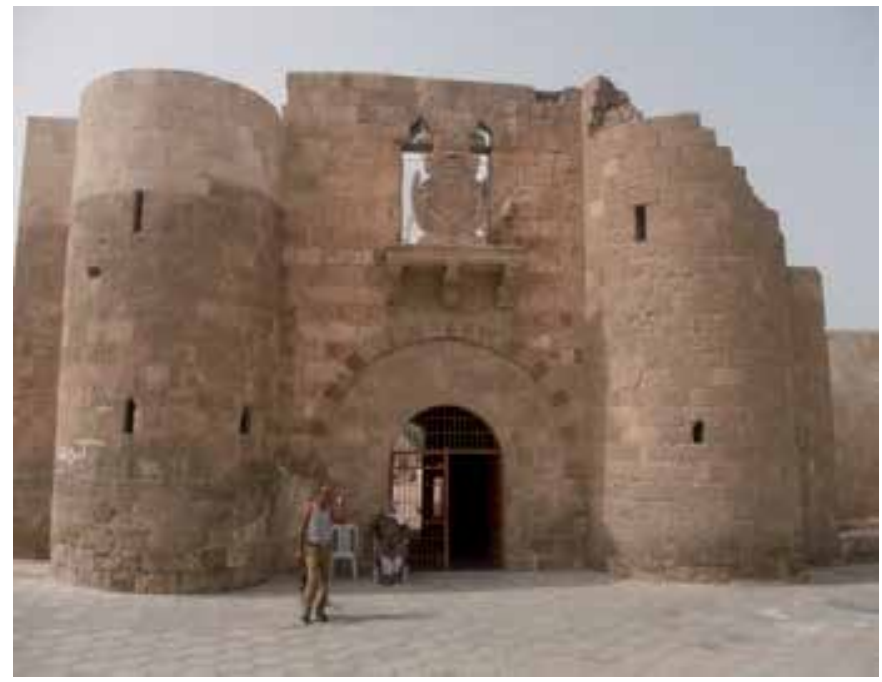

Fig. 2. Terrestrial photos for Aqaba fort.

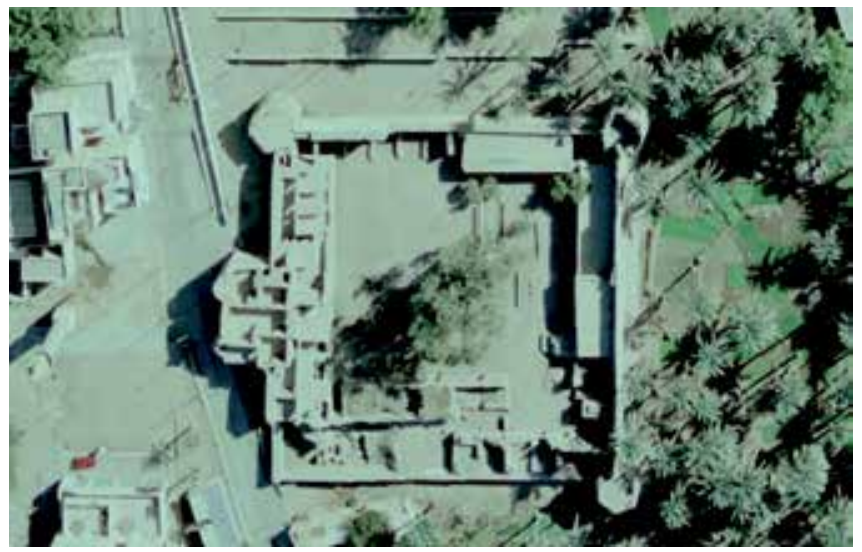

Fig. 3. Aerial view of Aqaba fort.

\section{Data collection}

The data sources starts with Photogrammetric data that include terrestrial, aerial and satellite images; second, spatial (GIS) data that include maps, vectors files such as ground control points, and location of point events, lines ( counter lines and roads). 


\subsection{Photogrammetric data}

The photogrammetric data includes: stereo aerial photographs, satellite images, LANDSAT and Spot images and terrestrial images. Two main photogrammetric products, Ortho-photos and digital elevation models (DEMs). Figure 4 describe sample of aerial photos captured 2007 and have 1/6000 scale used in Aqaba city. These photos have the following properties: Photo scale: (1:6000), film format is $(23 \times 23) \mathrm{cm}$, overlap between the two photos equal $60 \%$, Camera: Wild RC30 and Photography captured 2007.

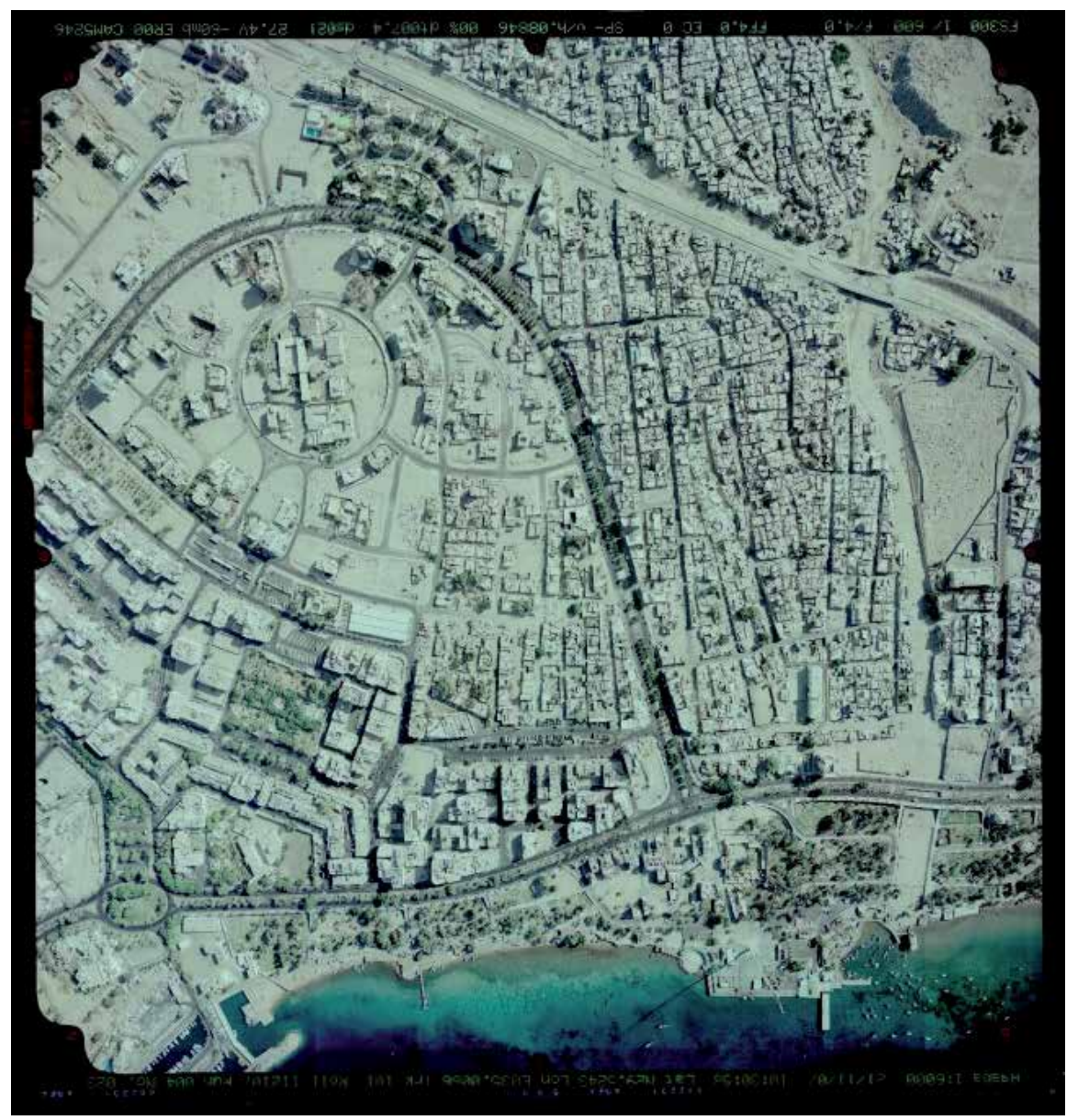

Fig. 4. Aerial photo of the study area.

Sample of images for landsat 2000 shown in Figure 5 has been used as base for GIS layers. 


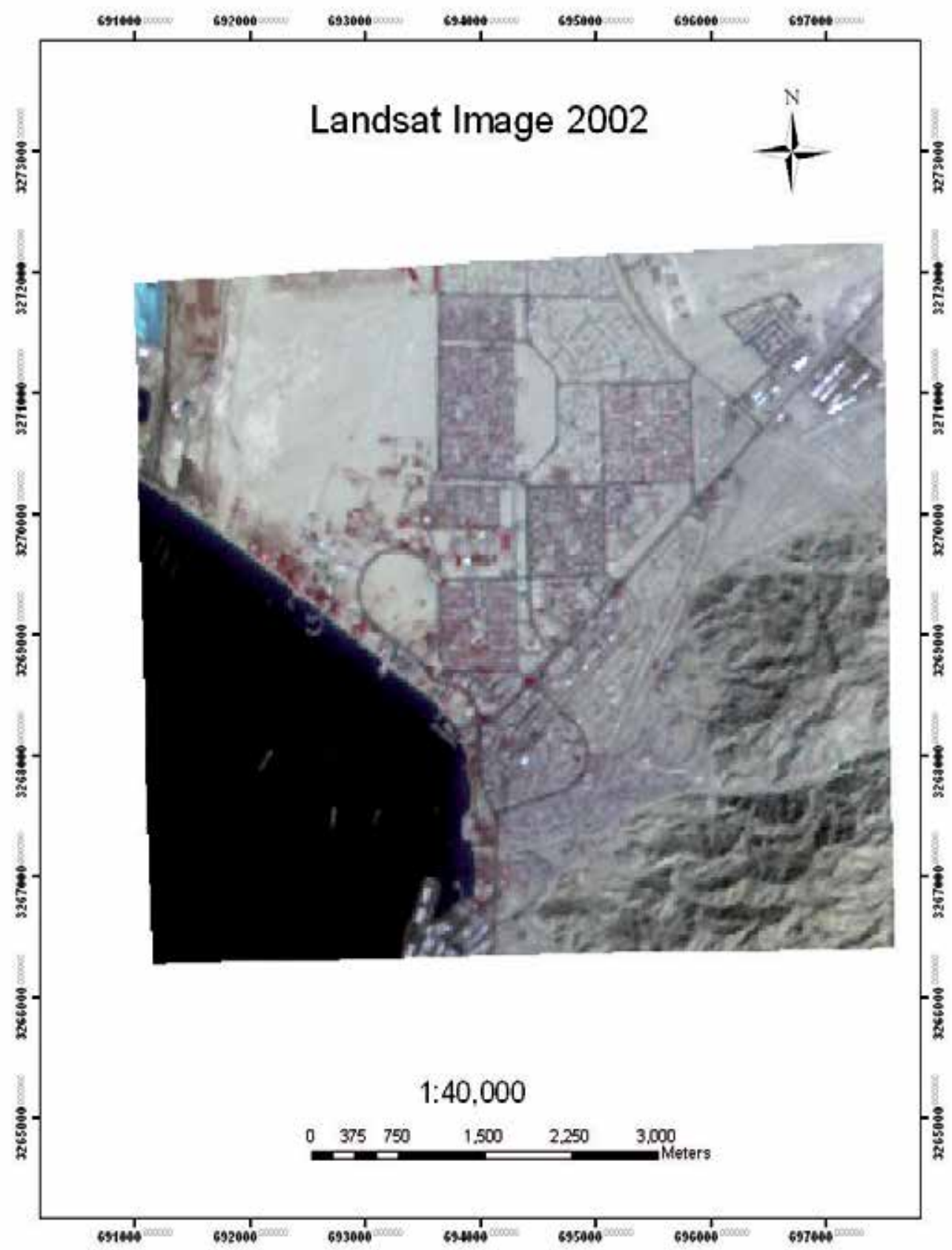

Fig. 5. LANDSAT 2000 image for the study area.

\subsubsection{Terrestrial Images}

Sample of photos with different angle of view captured for Aqaba appears in Figure 2. these images are used for building three dimensional model of front side of Aqaba fort. 


\subsection{Geospatial layers}

Basically most of layers used in this area is digitized from ortho-photo, such as building , roads and trees. The scanned hard copy maps appears in Figure 6, was captured from the fort museum and originally prepared by university of Victoria and Jordan ministry of tourism and antiquities at 2000 and 2005.

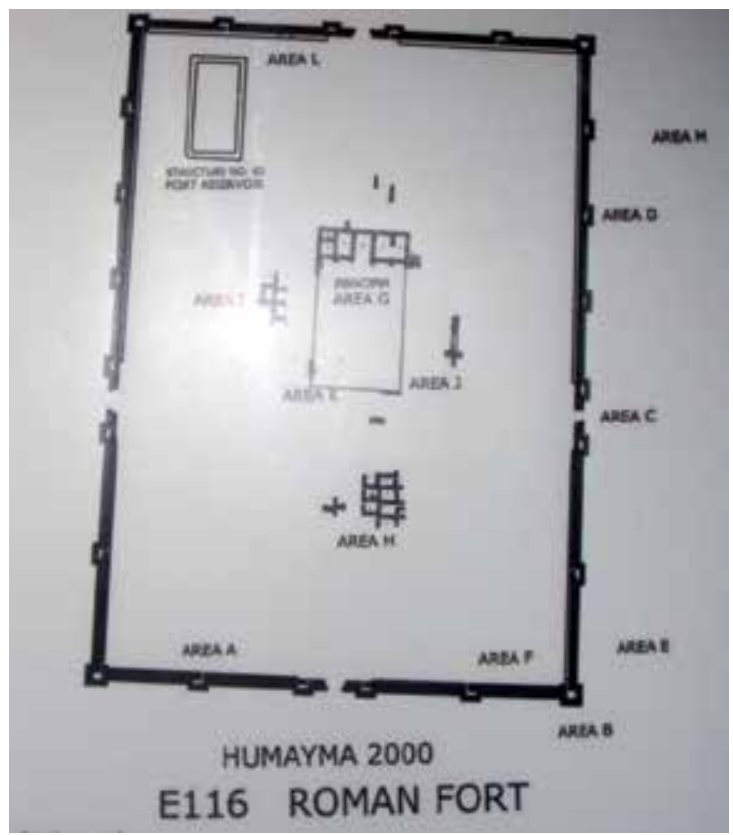

Fig. 6. Scanned hard copy maps for Aqaba fort.

$5 \mathrm{~m}$ Contour maps shown in figure 7, provided by Aqaba Special Economic Zone Authority has been used in this study.

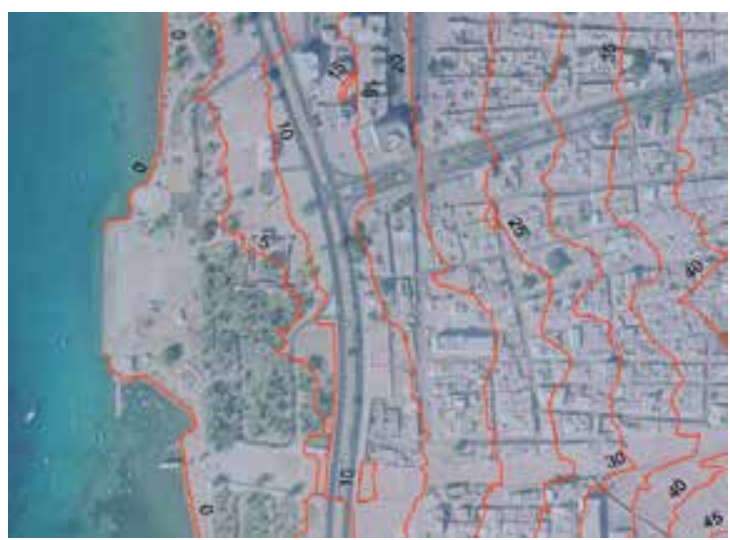

Fig. 7. Contour map overlay with Ortho-photo. 


\section{Data processing}

Different stages are included in this section starting with GPS for ground control reference information, photogrammetric processing to generate ortho-photo and digital terrain model, followed by GIS processing that include scanning, geo-referencing layer designing , digitizing and finally generation of maps and 3D representation of the site.

\subsection{Global positioning system}

Terrestrial and Global positioning system (GPS) survey were required within this study mainly for three major purposes: GPS survey was used to produce ground control points needed for processing of all aerial photographs and satellite images covering the archaeological sites; GPS survey also provided check points used for quality control of the produced ortho-photos and digital maps; and finally terrestrial survey was used to provide control and check points for the close range photogrammetry works.

All GPS survey of the ground control points was performed in static mode with sessions 10 minutes to $1 / 2$ hour. Check points collected with GPS were observed in Stop-and Go mode with usually 2 static reference receivers. The precision of a Stop-and Go GPS survey is about $10 \mathrm{~mm}+1 \mathrm{ppm}$ for post processed carrier phase observations.

The GPS field survey was processed using Ski-Pro and Leica GEO-Office software, while LisCAD Plus software was used for processing of the total station measurements. The following algorithm was followed in data processing: the coordinates of the reference stations (usually two) where obtained. The reference stations are points with known coordinates such as Ref BAU, the point at Al-Balqa' university or RAMO, which is one of the continuously operating reference stations of the International Terrestrial Reference Frame (ITRF). This point is closest to Jordan, it is coordinates are published on the official site of the International GNSS Service (IGS). The raw GPS observations for this station or any other of the ITRF are also provided by GNSS Data Center (GDC). For sites located away of available control points the coordinates of new reference stations are computed via OPUS-Online Positioning User Service, a web base service provided by the National Geodetic Survey of the USA. The control station coordinates and the GPS observations (including ground control point and checkpoints) were then used in the processing, which includes baselines processing, least squares adjustment (when redundancy available) and export of the final coordinates. The coordinates of all ground control points are computed in WGS84 coordinate system, ITRF 1994 or 2000, available in form of geodetic (latitude, longitude and ellipsoidal height) and Cartesian coordinates (Geocentric X, Y and Z). In order to produce maps and use the same points as references for the terrestrial survey these coordinates are transformed onto mapping surface using Universal Transverse Mercator Projection- zone 36 and 37. Additional control points are established during the total station survey in form of traverses linked to the GPS control points. Reference stations and GPS ground control points shown in Figure 8.

\subsection{Photogrammetric processing}

With the advent of digital photogrammetry and image processing technology, photogrammetric recording of world heritage sites has rapidly increased (M. Ioannides et 


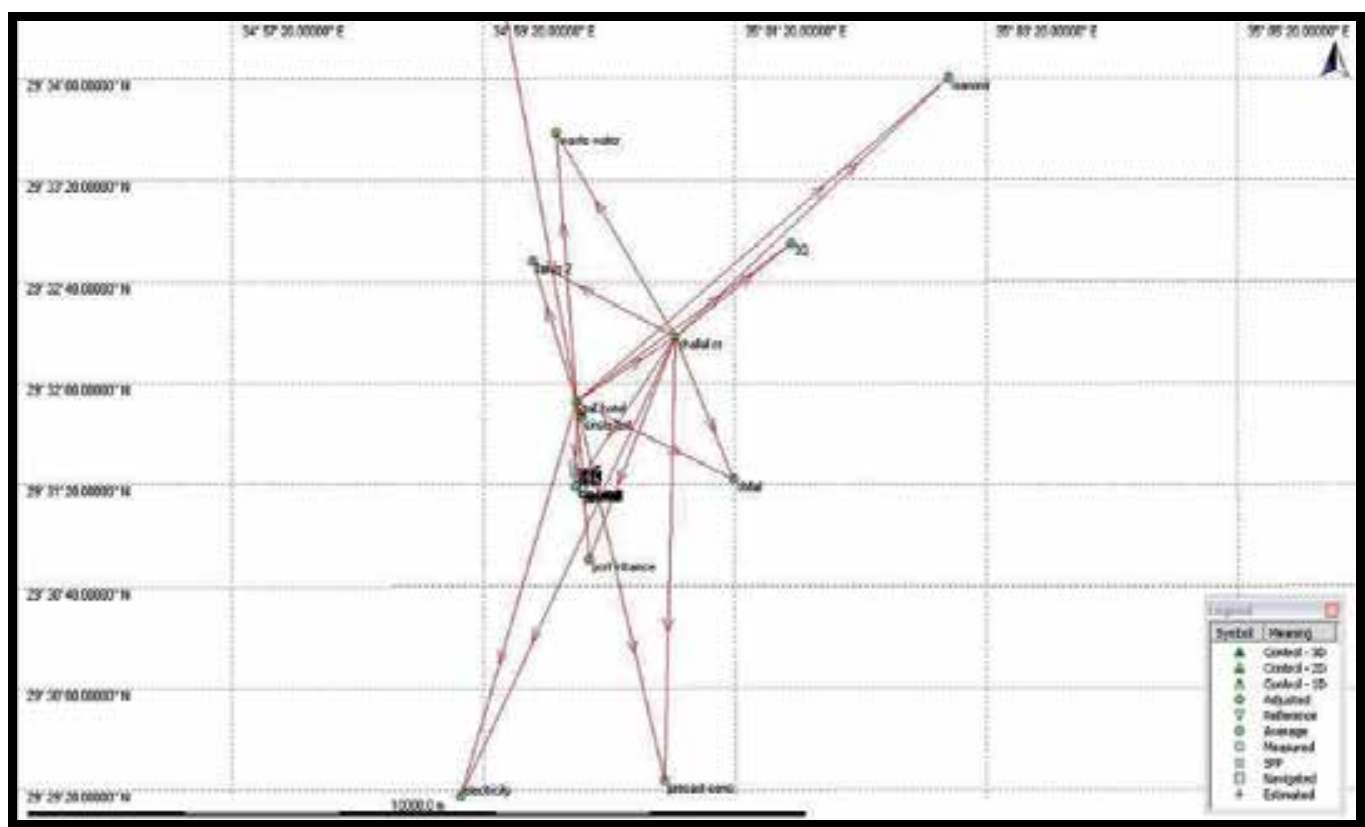

Fig. 8. Map for reference stations and GCP at Aqaba.

al.,2010). Digital object enhancement and 3D-modelling techniques are also possible and usually give clear presentation of heritage sites. They considerably enhance recognition of construction material, shape and area, and their spatial distribution, which is considered as one of the most difficult and time-consuming tasks for architects (Lerma et al., 2000). In summary, photogrammetry offers a rapid and accurate method of acquiring threedimensional information regarding cultural monuments. In this paper photogrammetric processing used for three main productions; Digital Terrain Model (DTM), orthophoto and real three dimensional models using close range photogrammetry.

The basic problem in analytical photogrammetry is to mathematically relate the positions in space of imaged objects to the positions of their image points in the plane of the image, and vice versa, (Kubik, 1991). The most basic geometric concept utilized in developing solutions to this problem is the collinearity condition which is that the camera station, the image point, and the imaged object all lie on a straight line. collinearity equations ( mathematical model between image and ground coordinate) appear in the form where the image coordinates of an object are expressed as functions of the Interior Orientation Parameters(IOPs) and the Exterior Orientation Parameters(EOPs), and the space coordinates of the object, (Krishna et al., 2008 ).

Several methods can be applied to determine the parameters of the orientation of one, two or more photos. The orientation can be processed in steps (relative and absolute orientation) and simultaneous methods (bundle adjustments) that is available in a majority of software packages. For systems based on stereoscopic measurements, the stereomodel derives from a relative orientation. In this step, points coordinates are determined in an arbitrary coordinate system of the stereomodel. The determination of points in the object coordinate 
system is done in a second step, known as absolute orientation, by applying a threedimensional similarity transformation.

For the interior orientation, two sets of parameters have to be considered. The first one contains the geometric parameters of the camera: the principal distance and the coordinates of the principal point. The second set includes the parameters that describe the systematic errors (as distortions or film deformations), ( Heuvel, 1999). at least 5 tie points ,that appears in the overlap area of stereo pair, required to extract the three dimensional model from stereo pair. Connection of an aerial Photograph and its corresponding object space is performed by the (EOPs) of that image. These parameters establish the physical geometry of imaging sensor with respect to ground and describe the position and orientation of that sensor at the time of imaging. At least, two full ground control points and one vertical is needed to solve the EOPs based on three-dimensional similarity transformation (Samadzadegan et al., 2002).

In this study two digital aerial stereo photographs were used to extract the ground coordinates of tie points and sequentially building DTM and orthophoto. Bundle adjustment within Leica Photogrammetry Suite (LPS) software has been used for modeling and DEM generation. The software supports reading of data, manual or automatic GCP and tie points collection and geometric modeling of aerial Photograph based on collinearity equations.

For DTM generation, image correlation strategy was used for automatic extraction of the tie points. Moreover, adaptive method was used to generate a DTM with grid format, where the topographic nature of terrain was considered; Firstly a DTM file was created with $10 \mathrm{~m}$ post spacing to provide the whole project area with initial elevations of the terrain model where each post was edited to ground and saved. Then another DTM was built with $5 \mathrm{~m}$ post spacing, where improved representation of real terrain for 1:5000 photo-scales can be obtained. Finally, The Root Mean Square Error (RMSE) was used to describe the consistency between the generated DTM and GPS points. The error is determined using the least square regression method. This is called the residual error that measures the fit in elevation between GPS points and extracted DTM . Eight check points were used to calculate the RMSE. The RMSE was calculated and found to be $0.351 \mathrm{~m}$. Figure 9 shows the DTM generated along for the study area where height ranges from 0 to 959 meter above mean sea level.

Next stage in photgrammetric processing is to generate an Ortho-photo as in Figure 10. Basically the Ortho-photo is a photograph transformed from perspective to orthogonal projection, or otherwise said, corrected for tilts and relief displacements. The produced DTM is to be used for Orthophoto generation. Regions that are not edited correctly in the DTM file appear on the Orthophoto as breaks and cuts in the region. These errors were removed by manual editing of the DTM based on visual inspections. Figure 10 shows the generated Orthophoto.

The last part in photogrammetric processing is the 3D modeling that deal with real representation of the features, shapes, and texture based on Close Range Photogrammetry. The principle of the 3D modeling is to give the features the same dimension and texture as reality (Wamg, Ahmad, 2002). 


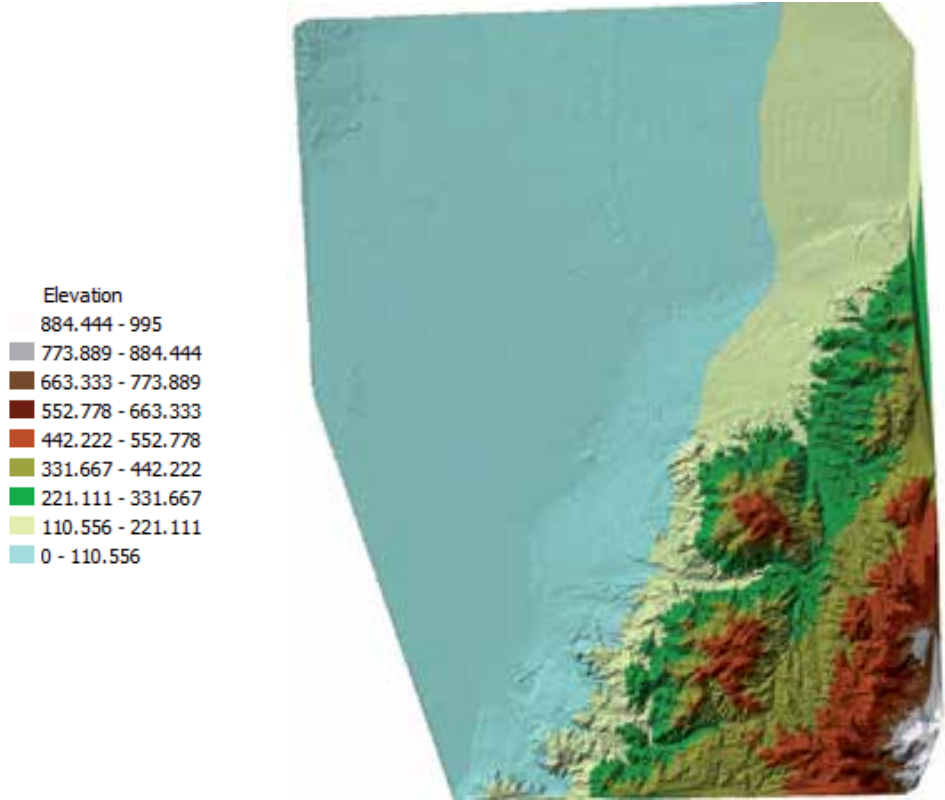

Fig. 9. Surface model for part of Aqaba area.

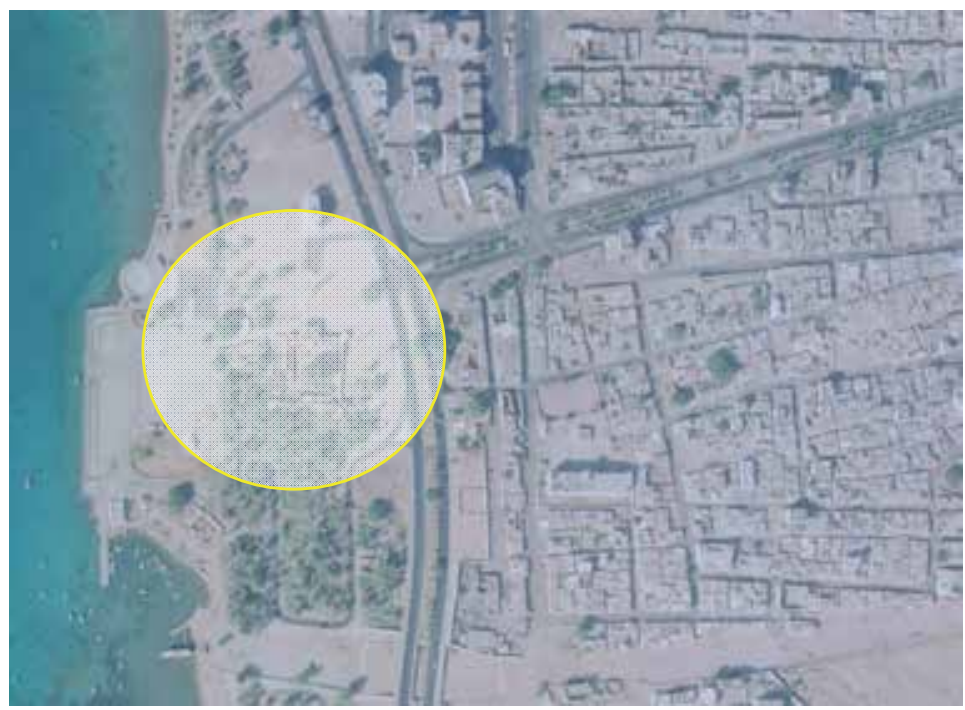

Fig. 10. Ortho-photo in Aqaba city with Aqaba Fort.

When using the photogrammetric techniques for recording and documenting the cultural heritage, the factors with impact on recording accuracy and archiving efficiency must be considered (Dallas et al., 2002): namely, metric characteristics of camera, imaging resolution and requirements of the bundle adjustment procedure. The mathematical model that incorporates self-calibration and bundle adjustment procedure for accurate estimation of the interior and exterior orientation parameters has been adopted. This is a necessary prerequisite for accurate and reliable 3D-reconstruction. 
After estimating the interior orientation parameters of the camera, experiments are conducted based on real data to build a three dimensional model. For this purpose, the calibrated SONY DSC-F707 digital camera is used to capture convergent images at three different locations with 90o rotation around the Z-axis at each exposure station. An arbitrary datum is chosen as reference for the object space, where conjugate points are selected. These measurements are introduced into the bundle adjustment in order to estimate their grounds synchronize. Selection of the points was performed while considering the following issues:

1. Distribution of points. The measured points must be well distributed and must cover the whole objects under study.

2. Visibility of each point in two or more images. If the same points appear in larger number of images, the geometrical strength as well as the accuracy of threedimensional coordinates will be improved.

3. Adequacy of selected points for the reconstruction of different shapes from an architectural point of view.

As shown in Figure 11, the three dimensional coordinates resulting from adjusting procedure are used to reconstruct 3D model for Aqaba fort that mentioned in Figure 2.

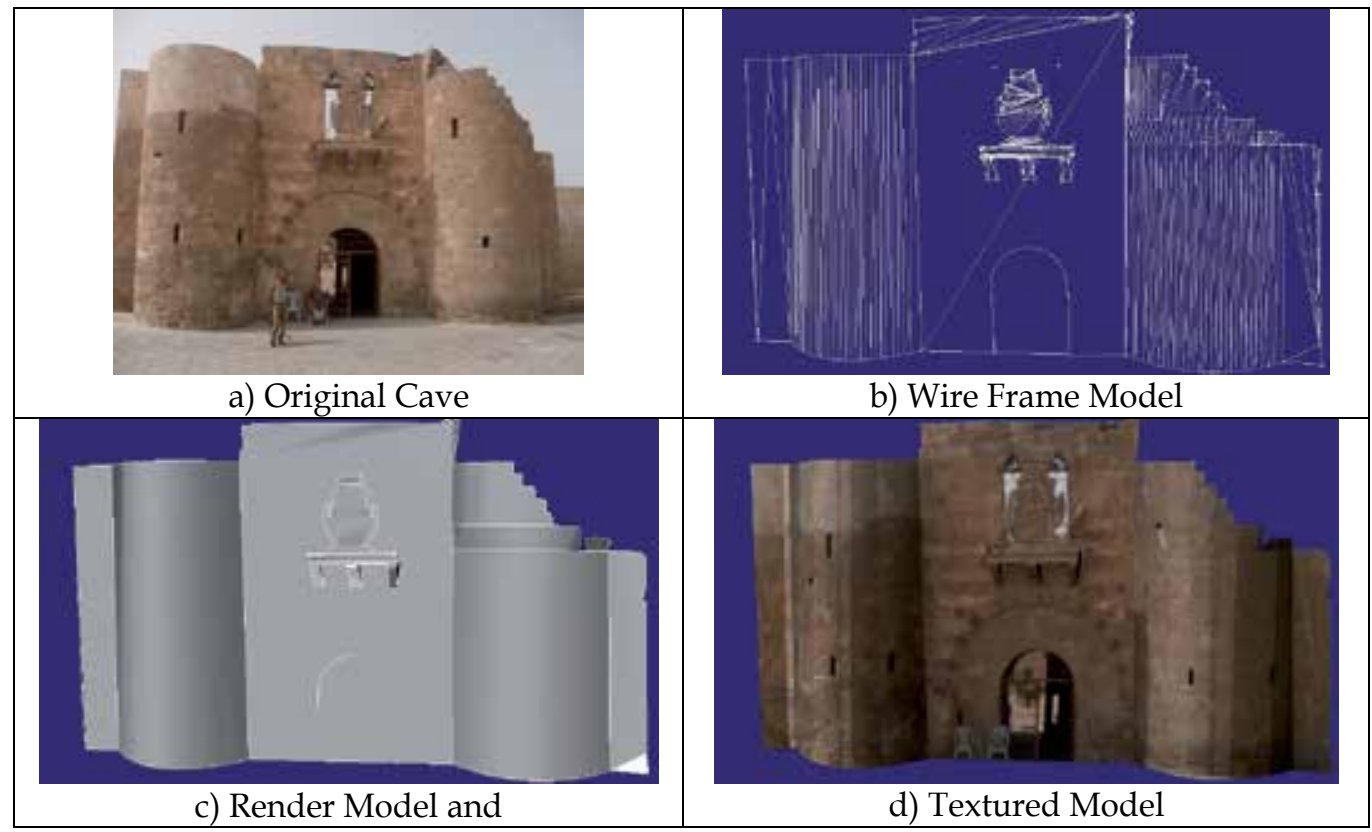

Fig. 11. Three Dimensional Model for the fort.

Surface rendering, which involves the generation of a 3D model with a real world surface texture, is constructed, Figure 11. That means, the surface textures are added to the 3D model surfaces to give a real world appearance to the displayed model. The 3D surface rendering is very important for the presentation of a ruined heritage sites where architects and renovation experts must have a realist view of the ruin for further inspiration (Ogleby 1999). Moreover, the 3D model can be digitally rotated to give a whole range of perspective views, (Habib et al., 2004).. 


\subsection{Geographic information system}

A Geographic Information System is a collection of information technology, data, and procedures for collecting, storing, manipulating, analyzing, and presenting maps and descriptive information about features to be represented on the maps (William et al., 1995). Cultural heritage objects often have a very irregular complex geometry. Thus, a good digital reconstruction requires a very detailed 3D model with a lot of geometry elements. So there are two main requirements for the 3D GIS. The first is a support for the acquisition and handling of large amounts of complex and non-planar 3D geometry. The second is the visualization of these objects which consists of a lot of geometry elements (Wüst et al., 2004).

This study highlights the representation and integration of photogrammetric products, which illustrated in previous sections in geo-referenced maps and images where the huge database can be manipulated, managed, and visualized. The obvious and accurate geometric overlaying of these different data will be helpful and great support for interpretation and analysis of the results.

It should to be mentioned that diversity of superimpose data that can be represented in the GIS system include (Boundary Maps, Point Location of Archeological Sites, Transportation Layer, Hydrological Maps, Digital Elevation Model, Aerial Images (Ortho-rectified), Satellite Images, Land Classification Image, Aquifer Profiles and Geology of the Area). All these layers are associated with database and descriptive attributes. The system should to be designed in a way that allow an easy and flexible updating of the new coming database. Figure 12 shows digitized features (Building, Streets, Parcels...) in GIS dropped over the digital elevation model.

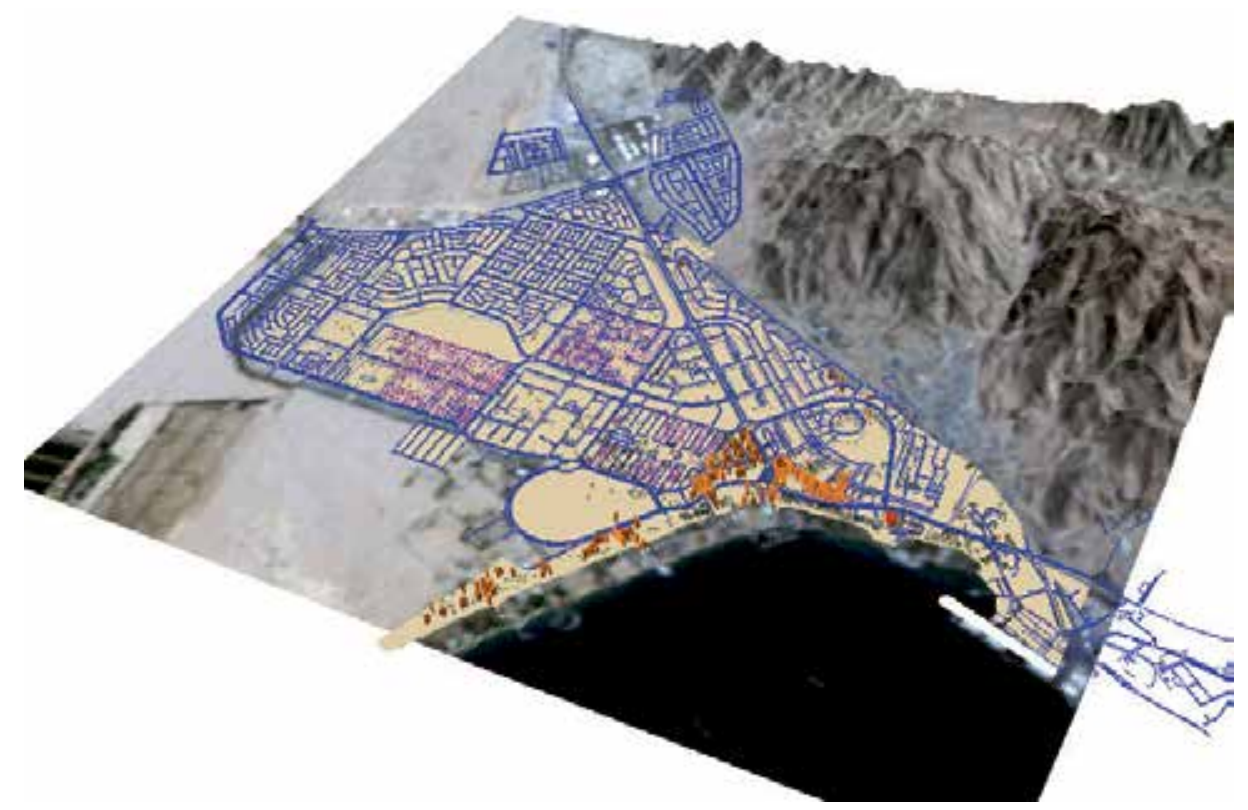

Fig. 12. 3D image with Digitized GIS features.

Figure 13 shows the terrain in three dimensional representations where figure 14 shows LANDSAT image dropped over elevation model. 


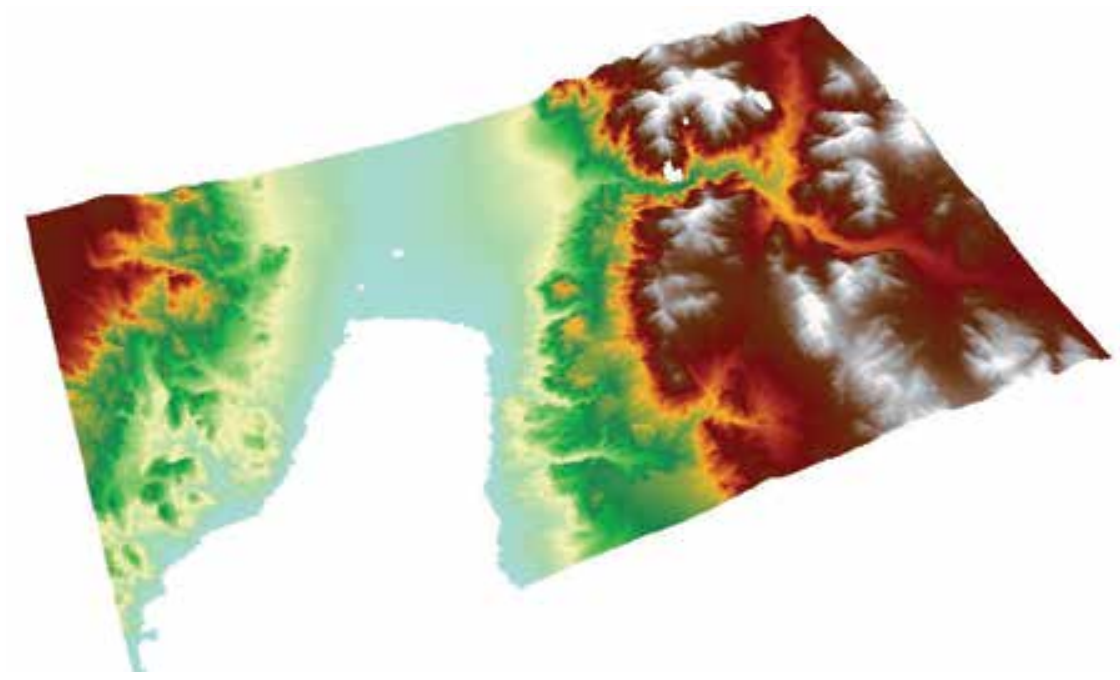

Fig. 13. Digital Elevation Model (DEM).

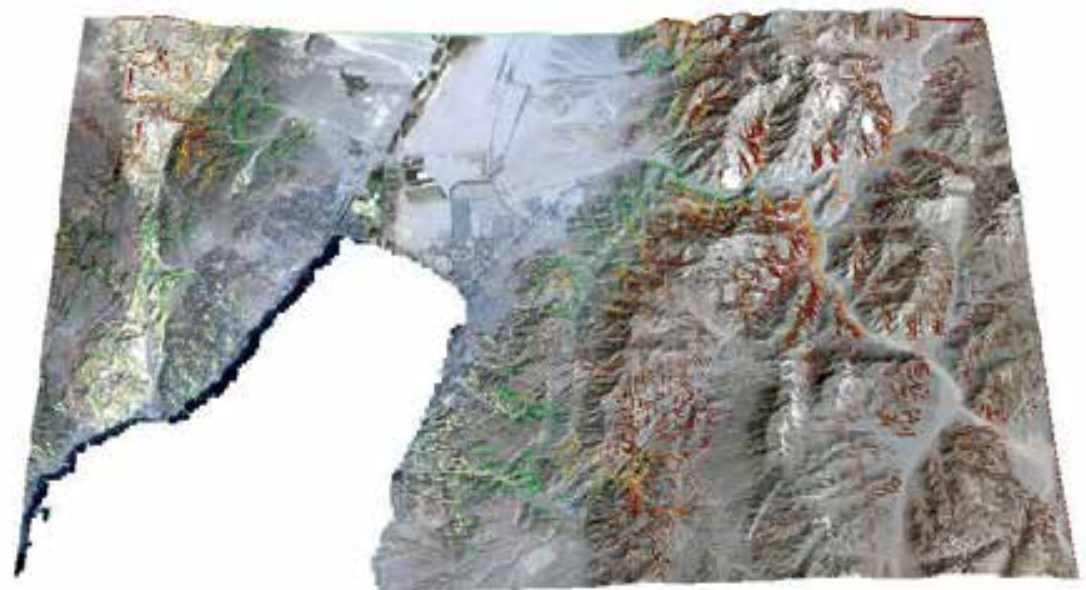

Fig. 14. LANDSAT Dropped On (DEM).

\section{Final remarks and future work}

In this study, we have presented different steps that can be used for archaeological documentation of Aqaba fort. The feasibility of integration between different sciences has been explored. Photogrammetric Techniques were used to emphasis on the identification of existing archeological sites in addition to generate map for tourism purposes where fair mapping of all historical objects can be conducted. Also, Aerial photographs Processing (stereo pair) in the study area was conducted where high resolution Orthophoto image produced in order to be used for measurement in GIS systems. Moreover, High resolution Digital Elevation Model was generated to grant details about the topography of the study area. Finally, 3D models of historical archeological structures in the fort was performed. During the generation of these models, requirements such as high geometric accuracy, availability of all details, and efficiency in the model size and photo realism have been considered. 3D models of historical 
archeological structures in the fort area with actual texture were generated. These models are vital for fair preservation of details of the historical castle.

Finally, photogrammetric as well as vector layers results have been geometrically registered and presented in a system that enable better viewing, analyses and interpretation. consequently, 3D GIS Model that include was established and serve as basis for preservations or for interactive presentations of the Aqaba fort.

\section{Acknowledgement}

This work has been supported by the Ministry of Higher Education and Scientific Research in Jordan, I would like to acknowledge their continuous support and generous funding.

\section{References}

Dallas, R., W., J. Kerr, S. Lunnon, and P. Bryan, 1995. Windsor Castle: Photogrammetric and archaeological recording after the fire, Photogrammetric Record 15(86):

El-Hakim, S., A. Beraldin and M. Picard (2002): Detailed 3D Reconstruction of Monuments using Multiple Techniques. In: ISPRS/CIPA International Workshop on Scanning for Cultural Heritage Recording, Corfu, Greece, pp.58-64.

Habib, A., Mwafag G R. Al-Ruzouq, and E. M. Kim 2004. 3-D Modelling of Historical Sites using Low-Cost Digital Cameras. XXth Congress of ISPRS, 12-23 July, 2004.

Heuvel, F., 1999. Estimation of interior orientation parameters from constraints on line measurements in a single image. Proceedings of International Archives of Photogrammetry and Remote Sensing, 32 (5W11): 81-88.

Krishna B., T. Amitabh, P .Srinivasan and K. Srivastava, 2008."DEM generation from high resolution multi-view data product". The International Archives of the Photogrammetry, Remote Sensing and Spatial Information Sciences. Vol. XXXVII. Part B1. Beijing: pp: 1099-1102.

Kubik, K., 1991. Relative and absolute orientation based on linear features, ISPRS Journal of Photogrammetry and Remote Sensing, Vol. 46, 199-204.

Lerma, J. Luis, Ruiz, L. Angel and F. Buchon, 2000. Application of spectral and textural classifications to recognise materials and damages on historical building façades. International Archives of Photogrammetry and Remote Sensing, 33(B5): 480-484.

M. Ioannides, D. Fellner, A. Georgopoulos, D. Hadjimitsis.Digital Heritage, 3rd International Conference dedicated on. Digital Heritage, ISBN 978-963-9911-16-1 Published by ARCHAEOLINGUA, Printed in Hungary by PRIMERATE, Budapest 2010, http://cipa.icomos.org

Ogleby, C.L., 1999. From rubble to virtual reality: Photogrammetry and the virtual world of ancient AYUTTHAYA, Thailand. Photogrammetric Record, 16(94): 651-670

Samadzadegan F., P. Ramzi and b. Schenk, 2008. "Aerial triangulation of digital aerial imagery using hybrid features". The International Archives of the Photogrammetry, Remote Sensing and Spatial Information Sciences. Vol. XXXVII. Part B1. Beijing 2008.

Wamg, and A. Ahmad, 2002. Digital architectural photogrammetric recording of historical building and monuments, New Zeland Surveyor, 293: pp. 25-30.

William E., and G. Allan, 1995."Manging Geographic Information System Projects". Oxford University Press, New York Oxford.

Wüst T., S. Nebiker and R. Landolt, 2004. "Applying the 3d GIS DILAS to Archaeology and Cultural Heritage Projects - Requirements and First Results". The International Archives of the Photogrammetry, Remote Sensing and Spatial Information Sciences, Vol. 34, Part. 


\title{
Underwater Photogrammetry for Archaeology
}

\author{
Pierre Drap \\ CNRS, French National Center for Scientific Research, \\ LSIS Laboratory UMR CNRS 6168 \\ France
}

\section{Introduction}

Archaeological excavations are often irreversibly destructive, so it is important to accompany them with detailed documentation reflecting the accumulated knowledge of the excavation site. This documentation is usually iconographic and textual. Graphical representations of archaeological sites such as drawings, sketches, watercolors, photographs, topography, and photogrammetry are indispensable for such documentation and are an intrinsic part of an archaeological survey. However, as pointed out by Olivier Buchsenschutz in the introduction to the symposium Images and archaeological surveys, in Arles, France, in 2007 (Buchsenschutz, 2007, Introduction page 5), even a very precise drawing only retains certain observations that support a demonstration, just as a speech retains only some arguments, but this choice is not usually explicit. This somewhat lays the foundation of this work: a survey is both a metrics document and an interpretation of the site by archaeologist.

The survey is a very important component of this documentation and its importance is largely due to the fact that the concepts employed by archaeologists during an excavation are closely related to space. The structure of the excavation is based on the concept of stratigraphic units. Inherited from geology and then formalized for archaeology by E.-C. Harris (Harris, 1979), stratigraphic units are linked by geometric, topological and temporal relationships. They are fundamental for the interpretation of the archaeological excavation.

Two families of objects have to be surveyed: first, the artifact that we seek to position in space and of which we have a good a priori knowledge; and second, the area being excavated (in this case, a part of the seabed), often represented as a digital terrain model (DTM). Throughout this work we deal with these two aspects, artifacts and unstructured land, by addressing two different approaches; one using a priori knowledge through measurements and the second based solely on geometry.

The first approach, based on the a priori knowledge that we have about the measured artifact, uses our knowledge of the object to compute its size and position in space. This method can also reduce the time required for measurements.

The second approach, used to survey land for example, uses automatic tools coming from photogrammetry to compute a dense cloud of 3D points. 
Finally, a very important point is the link between geometry and knowledge; a model, 3D or $2 \mathrm{D}$, representing a site is a relevant interface to access the data known about the site. $3 \mathrm{D}$ representations of a site provide important added value to underwater archaeologists who are then able to study a three-dimensional overall picture, which is otherwise difficult to obtain in underwater environments. This offers a significant advantage in the creation of new assumptions regarding the reconstitution of the shipload or vessel itself (Hesnard, 1988; Long, 1995).

Moreover, it should be noted that, by nature, archaeological data are incomplete, heterogeneous, discontinuous and subject to possible updates and revisions. The documentation system, linked to archaeological data, must be able to manage these constraints.

\section{Underwater photogrammetry: More than $\mathbf{5 0}$ years of experience}

The invention of the pressure regulator (Aqua-Lung) in the 1950s enabled scuba divers to reach underwater archaeological sites and gave them the possibility of studying the wrecks of sunken ships. After the first experiments in the Fifties (of which the "excavation" of Grand Congloué by Jacques-Yves Cousteau and Fernand Benoit represents a milestone; celebrated, but unfit from the scientific point of view), in the Sixties archaeologists understood that they had to dive themselves to ensure that the terrestrial scientific methods used for underwater sites were correctly applied. The terrestrial methods of excavation, based on principles coming from geology and prehistoric archaeology (stratigraphic excavations), were applied by using tools adapted to the sea environment, such as suction dredgers powered by air or water, in order to remove sediment without moving the objects constituting the load of a wreck, and by excavating layer by layer the hull of the ship. On the other hand, the methods of terrestrial graphic statements (horizontal planes, vertical cuts) could not be adapted in a simple manner as they were based on optical tools, theodolites, levels, etc. Traditional methods such as tracing right angles or placing test cards on a horizontal plane were possible, but the manual graphic statement was not adaptable to the excavation of an important cargo of amphorae, ending up to be much too slow and vague without the use of a theodolite.

The first experiments of underwater photogrammetry for archaeology began in the Sixties (Bass, 1970). In the same decade, the Naval Oceanographic Office in Washington performed the first experiment with underwater photogrammetry on a submarine under the direction of Joseph Pollio (Pollio, 1968). Afterwards, other experiments took place involving the basic concepts that we use today (Ciani et al., 1971; Faig, 1979; Hoehle, 1971; Pollio, 1971). Since then, the interest in photogrammetry and acoustic measurements for archaeology has continued to grow (Bass \& Rosencrantz, 1973; Tchernia et al., 1978).

In 1964, the submarine Asherah, with financial support from the National Geographic Society, was inaugurated in Turkey, and by $35 \mathrm{~m}$ deep took the first-ever stereoscopic photographs of the Yassi Ada 2 Byzantine wreck (Bass, 1970; Bass \& Rosencrantz, 1973). A large mechanism was needed to achieve these stereoscopic images. The photogrammetric survey of a wreck in France was performed in Marseille by the French archaeologist Bernard Liou (Liou, 1973). While two synchronized cameras were taken on board the submarine Asherah, the diver in Marseille surveyed the site using fixed and sliding metal structures. 
This framework was used on site to guide the cameras shooting the stereoscopic images. It was subject to strong geometric constraints; images were to be made in accordance with human physiological conditions required for stereo vision: parallel optical axes and no tipping (rotation around the optical axis).

\section{Underwater survey}

Studies on aboveground archaeological sites, as well as shipwreck sites, have always aimed to obtain very accurate graphical representations of reality. As soon as appropriate tools became available, a trend towards obtaining 3D representations on graphic displays also developed.

Due to their location in the open sea, where several factors such as darkness, low temperature and low oxygen rate are combined to help ensure wreck preservation, underwater sites offer extraordinary opportunities for archaeologists. However, deep wrecks are now jeopardized by an increase in deep trawling that destroys the surface layer of the sites and thus scrambles legibility. Indeed, the twenty-year-old assumption that deep wrecks would be protected from trawling is no longer true as trawl nets can nowadays be deployed down to depths of $1000 \mathrm{~m}$. Therefore, many of these wrecks are likely to be destroyed even before they can be studied

The generation of thorough and exhaustive 3D records of these wrecks consequently gains importance daily. As these sites can never be experienced firsthand by the majority of archaeologists or the general public, it is very crucial to provide a faithful and accurate 3D survey of the site which can provide virtual access to all archaeological data.

\subsection{Underwater 3D survey merging optic and acoustic sensors}

Optic and acoustic data fusion is an extremely promising technique for mapping underwater objects that has been receiving increasing attention over the past few years (Shortis et al., 2009). Generally, bathymetry obtained using underwater sonar is performed at a certain distance from the measured object (generally the seabed) and the obtained cloud point density is rather low in comparison with the one obtained by optical means.

Since photogrammetry requires working on a large scale, it therefore makes it possible to obtain dense 3D models. The merging of photogrammetric and acoustic models is similar to the fusion of data gathered by a terrestrial laser and photogrammetry. The fusion of optical and acoustic data involves the fusion of 3D models of very different densities - a task which requires specific precautions (Drap et al., 2005; Hurtós et al., 2010).

Only a few laboratories worldwide have produced groundbreaking work on optical/acoustic data fusion in an underwater environment. See for example (Singh et al., 2000) and (Fusiello \& Murino, 2004) where the authors describe the use of techniques that allow the overlaying of photo mosaics on bathymetric 3D digital terrain maps (Nicosevici et al., 2009). In this case we have important qualitative information coming from photos, but the geometric definition of the digital terrain map comes from sonar measurements.

Merging optical and acoustic survey is also done with structured light and high frequency sonar by Chris Roman and his team (Roman et al., 2010) this approach is very robust and accurate in low visibility condition but don't carry qualitative information. 


\subsection{Underwater photogrammetry}

The principle of underwater photogrammetry does not differ from that of terrestrial or aerial photogrammetry, but it is necessary to take into account certain elements that may cause disturbance, in particular the refraction of the diopter water-glass and the presence of the housing (Butler et al., 2002).

The specific constraints of the underwater medium (turbidity of water, presence of suspended particles) force the operators to work on large scales, close to the objects (between 0.5 and 2 to 3 meters, depending on the water quality). This apparently constraining aspect imposes a great quantity of stereotypes to us, but on the other hand it offers a very high degree of accuracy.

The important advantage of using photogrammetry in underwater surveys in comparison with the use of other techniques consists in its simplicity of implementation and the diversity of potential results (3D measurements on the object, 3D reconstruction, orthophotography, and vector restitution).

The implementation only requires the use of a scale bar to compute the scale of the model. Moreover, if two or three synchronized cameras are used, additional equipment is not needed on the scene as the scale is computed using the calibration of the camera set. This approach also provides a relevant appreciation of the uncertainty of measurements; where, in addition, the photographs have to be taken with an important overlap. The key factor of this method is redundancy: each point of measured space must be seen in at least three photographs.

The operative advantage is related to the simplicity of the survey. Moreover, a submarine pilot can drive a remotely operated underwater vehicle (ROV) without having to undergo a long preliminary training period. This method requires little time and does not require specific personnel, thus greatly reducing the expenses in a context where time and costs of intervention are extremely high.

\subsection{Underwater camera calibration}

Camera calibration in multimedia photogrammetry is a problem that has been identified since almost 50 years ((ASP, 1980) page 838). (Bass \& Rosencrantz, 1973; Fryer \& Fraas, 1986). The problem has no obvious solution, since the refraction of the light beam through the different media (water, glass, and air) introduces a refraction error which is impossible to express as a function of the distortion of the image plane coordinates alone (Lavest et al., 2003). A clear presentation of this problem is related by Hans-Gerd Mass and also Mark Shortis (Maas, 2000) (Doucette et al., 2002; Shortis et al., 2007).

Telem and Finlin (Telem \& Filin, 2010) propose a model which uses collinearity equations to integrate the deviation caused by refraction on a plane interface. The results are interesting but the bundle adjustment cannot be made using traditional software designed for terrestrial surveys.

Therefore the deviation due to refraction is close to that produced by radial distortion even if radial distortion and refraction are two physical phenomena of a different nature. For this reason, the approach described by Kwon (Kwon, 1998) has been adopted, consisting in the 
use of standard photogrammetric calibration software to calibrate the digital cameras and their housing. This approach can indeed correct in a large part the refraction disturbance; however, it is strongly dependent on the optical characteristics of the water/glass interface of the housing. In order to minimize the refraction error due to this last interface, a housing with a hemispherical glass must be used (Telem \& Filin, 2010).

\subsection{Bundle adjustments}

An estimation (or adjustment) technique is required when the number of available measurements is greater than that necessary to obtain a uniquely determined solution for a set of unknown parameters. From a number of estimation methods available, that of least squares is utilized most often. In many fields of science and engineering, including surveying, geodesy and photogrammetry, least-squares estimation is used extensively. (K.W. Wong, in (ASP, 1980) p. 97).

The adjustment of photogrammetric networks is accomplished via the least-squares process known as the bundle adjustment. This facilitates the simultaneous estimation of object point coordinates and image exterior orientation parameters.

The bundle adjustment incorporates various computational techniques to improve efficiency. At the same time, the goal was to make the procedure as general as possible in order to address the various requirements encountered in close range photogrammetry. These include the use of control points when available, the lack of control points (which is more typical in an underwater environment), and the implementation of constraints between points such as distance or axis constraints (Edmundson \& Fraser, 1998, Edmundson, 1997).

Three dimensional network adjustment problems have an inherent rank deficiency of seven, called the network or datum defect. The datum defect exists if the network is not attached to a known coordinate system. The defect of seven includes three each for position and orientation, and one for scale.

The datum defect can be overcome by arbitrarily "fixing" the network in position, scale, and orientation through the use of control points, or points with known coordinates. The minimum number of fixed coordinates required is seven, usually obtained from two full control points and a third fixed in only one coordinate. Adjustments utilizing exactly enough constraints to define the datum are referred to as minimal constraint solutions.

Often however, particularly in terrestrial applications such as those found in archaeology or industrial photogrammetry, reliable control points are not available. Another means of eliminating the datum defect is through the inner constraints approach to free network adjustment (Blaha, 1982; Fraser, 1982).

The free network solution is optimal in that the mean variance $\left(\bar{\sigma}_{c}^{2}\right)$ of the object point coordinates is minimized. In addition to its value when there are no control points, the free network is also advantageous for an accurate analysis of observation residuals. The use of redundant control can introduce stress into an adjustment which can, in turn, mask blunders that may otherwise be discovered in the residuals (Blaha, 1982). 


\section{Measuring artifacts, the use of archaeological knowledge}

The innovative approach developed to measure in situ archaeological artifacts relates to integrating measured data with a theoretical model in order to obtain a complete representation of a partially measured object. The way to merge the different data is formalized in logical rules and for each instance measured, an expert system, fully integrated in the photogrammetric software, determines a set of operations needed for the geometrical computation.

The approach, presented in this section, was first implemented on an underwater archaeological excavation of the Grand Ribaud F, a deep Etruscan shipwreck discovered in 1999 in Hyères, France. The wreck is of high archaeological interest for both its cargo and its state of conservation. It is about 2,600 years old and it contains nearly 1,500 amphorae (Drap et al., 2003).

\subsection{Merging theoretical model and measure}

From the oriented photographs, a subsequent 3D geometrical modeling phase of the recorded artifacts (amphorae in our case) is performed. In this phase, the modeling must be driven by expert (archaeological) knowledge; thus the plotting phase is done manually, by an expert who inserts annotations to the measured geometry. The resulting models, together with the photogrammetric georeferenced data and all the survey data, are stored in a repository database for further use and interrogation.

The 3D modeling phase procedure consists in exploiting archaeological knowledge to obtain a complete representation of the measured artifacts. It is articulated in two steps:

1. Development of the theoretical model: for each identified object, a geometrical description offers a set of geometrical primitives, which are the only features to be potentially measured (see figure 1); these are compared with the theoretical representation of the object as derived from expert knowledge. During the site study, archaeologists have to identify amphora typologies, and a theoretical model is produced for each of them. This theoretical model is formalized in a hybrid way, using both taxonomy of archaeological artifacts and XML representation for the amphora typology.

2. Decision Support System: as photogrammetric measurements are highly incomplete (the object is only partially visible or may be deteriorated), a rule-based Expert System determines the best strategy to provide all the geometrical parameters of the studied object, starting from the measurement process to the handling the default data as defined in the archaeological and geometrical models. In our case, we use the Jess expert system (http://herzberg.ca.sandia.gov/jess/).

The resulting object is thus based on a theoretical model, dimensioned by a photogrammetric measurement (see figure 2, 3 and 4). The modeling procedure is revisable in time, allowing re-processing or complementary processing as new data becomes available. The whole procedure is implemented in the Java programming language and can be used with the ARPENTEUR photogrammetric toolbox (Chapman et al., 2010; Drap et al., 2003; Drap \& Long, 2005; Drap et al., 2008).

Amphorae classification in archaeological work very strictly relies on dimension information for specific features of the object; for instance, the neck or belly. In providing a 
theoretical model for a specific amphora class, it makes sense to measure these features directly on available archaeological finds.
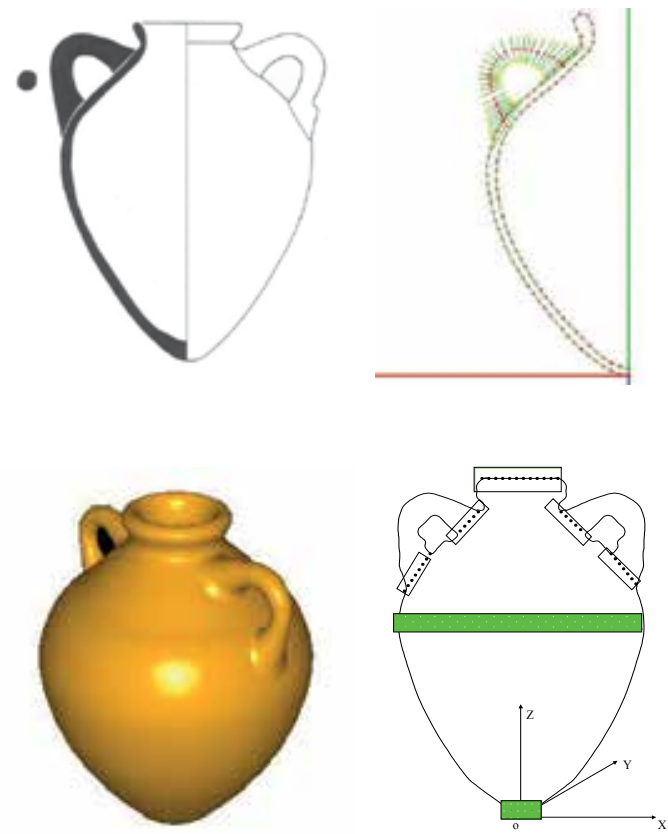

Fig. 1. On the left, the graphical model designed until now by archaeologists. On the middle two images, a CAD interpretation of this model. On the right, the five zones where photogrammetric measurements can be taken on an amphora.

In defining the theoretical model, the diversity of the objects handled by the archaeologists and the geometric complexity of their surfaces led us to search for stable morphological characteristics of the objects where diagnostic measurements could be taken. A series of simple geometric primitives are used to approximate these morphological characteristics and to act as an interface between the photogrammetric measurement and the underlying model. In the case of amphorae, four measurable zones have been defined: rims, handle, belly and bottom (see in figure 6 measured points in green on the final complete amphora model). The least-square method is used to fit a set of simple geometrical primitives onto the measured points; for instance, a circle on the rim or belly points, a line on bottom point, etc. This interface allows the user (generally an archaeologist) to

- Recognize the amphora type in the photographs;

- Choose the amphora type in the interface combo box ;

- Measure a set of points in the zone where a measurement is possible;

- Add archaeological comments and observations;

- Ensure consistency between observations and the theoretical model;

- $\quad$ Store a new instance in the database. 


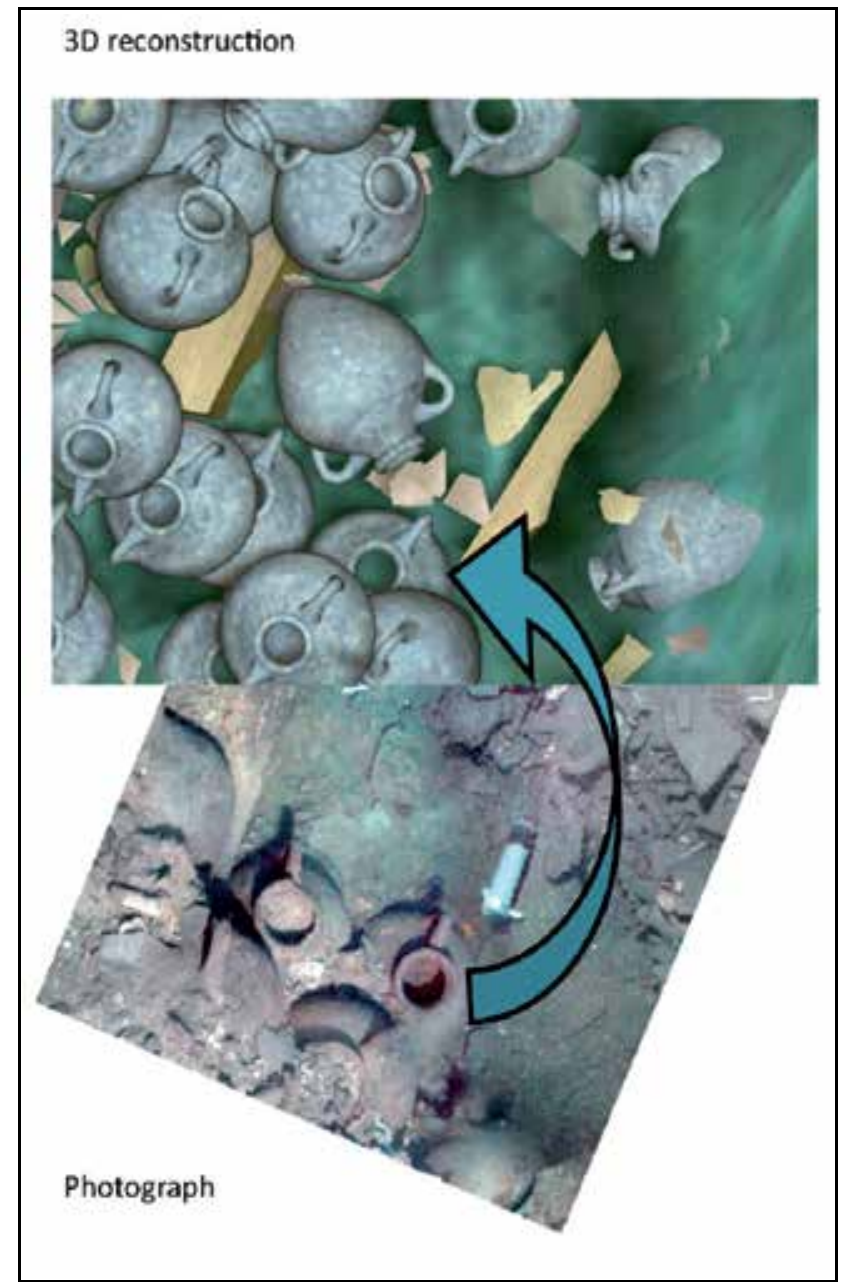

Fig. 2. On the top, a complete virtual visualization of the site of the Grand Ribaud F wreck with all the amphorae measured. On the bottom, one of the original photographs used for the photogrammetric survey.

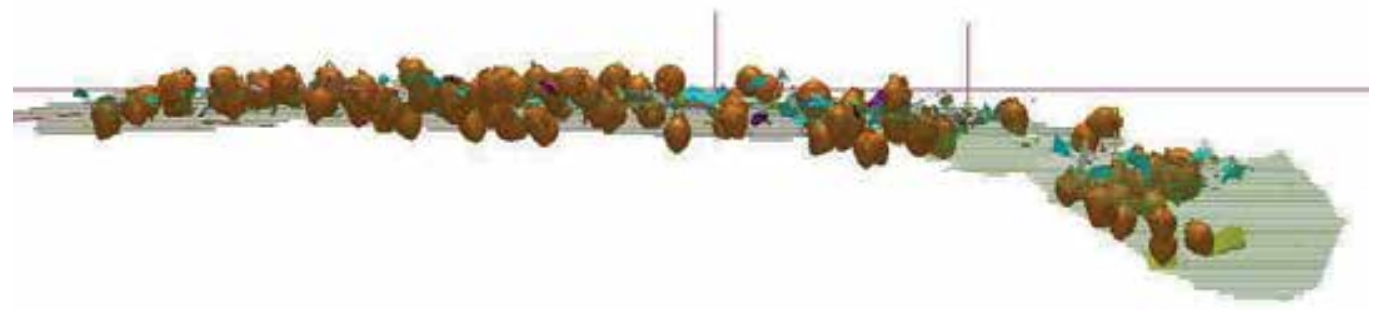

Fig. 3. An east-west elevation cut of the excavation made on the Grand Ribaud F wreck visualized using MicroStation ${ }^{\mathrm{TM}}$. 


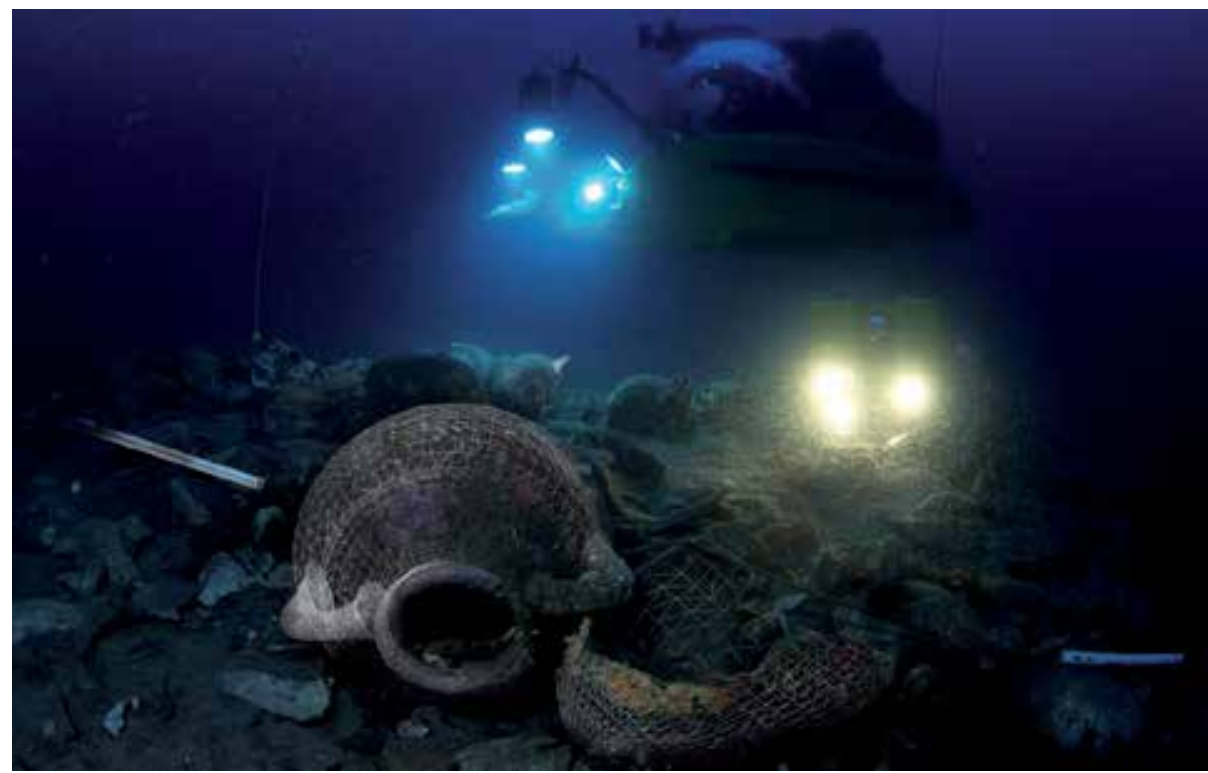

Fig. 4. The Grand Ribaud F wreck: a digital model of an amphora (Etruscan Py4) inserted into a photograph. (Original photograph from F. Bassemayousse).

\subsection{Inconsistency check}

During a survey, measurement errors can occur. The measurements of an object can be erroneous due to a poor photogrammetric restitution as it is difficult to clearly see all the objects in a photograph. For a large site survey (with large dimensions or with a large amount of objects), many surveyors can be involved in different measurement sessions. In this case, the same object may be measured more than once and different objects can be assigned the same identifier. These errors are a part of the possible errors that can occur during the construction of the final result of a survey.

For large surveys, an automatic way to ensure the consistency of a result is needed because users cannot verify by themselves all the measured objects. Such an automatic method relies on two major points:

- Verify that a set of objects is consistent

- Show inconsistent objects and origins of inconsistency to the surveyor

Using this method, the surveyor has only to correct the inconsistent objects without having to check them one by one.

The verification of the consistency is based on the knowledge of the experts in the domain. A theoretical model is constructed from expert knowledge. This model contains a description of the object (class, geometry and default values) used during the measurement sessions. From the knowledge of the experts we can also extract some constraints on the measured objects.

For example, the height $h$ of a tile cannot exceed or be under $20 \%$ of its default value $h_{d}$. If $h$ is the height of an amphora, the constraint can be expressed: 


$$
\left|\mathrm{h}_{\mathrm{d}}-\mathrm{h}\right|<0,20 \mathrm{~h}_{\mathrm{d}}
$$

A constraint that involves only one object is called an intrinsic constraint. For each object typology, we have a set of intrinsic constraints given by the experts.

Constraints do not only apply to a single object. Experts can provide constraints that apply to a set of objects. Spatial constraints are a good example of constraints that involve more than one object. A spatial constraint could be the 3D representations of two amphorae that do not intersect. This constraint relies on the existence of a relation between two objects that determines if the two 3D representations intersect. Constraints that involve relations between objects are called extrinsic constraints.

The verification of the consistency of a set of objects relies on the definition of the typology of the measured objects and the set of intrinsic and extrinsic constraints. We propose a formal representation, called Entity representation, designed to verify the consistency of a set of measured objects. For more details on this approach, please refer to (Papini \& Drap, 2009; Seinturier, 2007; Sérayet et al., 2011).

\subsection{Accuracy}

Accuracy estimation must still be improved. The first observation we can make is that the accuracy is proportional to the resolution and the scale of the photographs.

The behaviour of light underwater makes it necessary to take photographs close to the object, which ensures having a good scale. The use of a good quality digital camera with correct lighting (generally the use of a good strobe system) will ensure the best conditions to obtain good accuracy.

The general accuracy depends of two families of conditions: the geometry of the block in regards to its orientation and the way to identify homologous points on several photographs.

Regarding the geometry, it is obvious that ribbon geometry is not relevant. Overlapping between photographs and strips is important, but additional images using a smaller scale can also help fix the block orientation.

Concerning homologous point detection, a well-textured surface is important and as well as avoiding points close to the border and discontinuity. Note that automatic homologous point detection and matching using the SIFT (Lowe, 2004) or SURF (Bay et al., 2008) algorithm can be improved by image pre-processing (Kalia et al., 2011).

Nevertheless, the problem remains to quantify the accuracy. A standard estimation of variance and covariance does not seem relevant. A simple analysis of the discrepancy between the measured position and the projection of the 3D computed points onto the original photograph gives a good idea of the general accuracy of the block orientation.

If the survey is performed with only one digital camera, we need exterior information to scale the model. This can be done with a set of simple scale bars layered on the site, For example on the Grand Ribaud F survey, visible in Figures 2, 3 and 4, a visual verification using a $2 \mathrm{~m}$ scale bar, not used to orient the model, gives an estimation accurate to $2 \mathrm{~mm}$. 
In certain cases the camera can be mounted on an underwater vehicle and it is possible to benefit from the navigation data to obtain an approximation of the camera orientation and scale. Often in underwater archaeology, a survey using a multibeam sonar system is performed to obtain a small-scale representation of the site and its surroundings.

The availability of acoustic bathymetric data from the multibeam survey, which was the case in the missions organized during the VENUS (Virtual ExploratioN of Underwater Site) project (Drap et al., 2008) allows for a comparison with the final 3D model obtained with the photogrammetric approach. Nevertheless, it has to be mentioned however that the resolution of the multibeam survey is of the order of 1 sample every $0.5 \mathrm{~m}$, over a large area, while the resolution of the photogrammetric data is approximately 1 sample every $0.01 \mathrm{~m}$ over a much smaller area. The discrepancy between the digital terrain models (DTM) obtained from the multibeam survey and the photogrammetry in the $\mathrm{Z}$ direction shows a mean systematic error of $0.502 \mathrm{~m}$ with a root mean square (RMS) of $0.073 \mathrm{~m}$.

\subsection{Implementation on site}

The survey on the Grand Ribaud F, as well as on the VENUS missions presented here, was done using a single digital reflex camera, Nikon D300 or D700, and two Ikelite strobes. The camera can be mounted on an underwater vehicle or handled by a diver, as we are sure that the seabed can be represented by a DTM (no cave, no wall). We can use a set of photographs with a vertical reference to perform the survey. The photographs are taken in strips with $60 \%$ overlap for the consecutive photographs in strips with $20 \%$ overlap from one strip to another.

Using only a single camera and no acoustic survey equipment means that we do not have an a priori knowledge on the orientation of the photographs and we need at least a set of scale bars. Without other information, we use buoys (see Figure 5) to define a vertical axis.

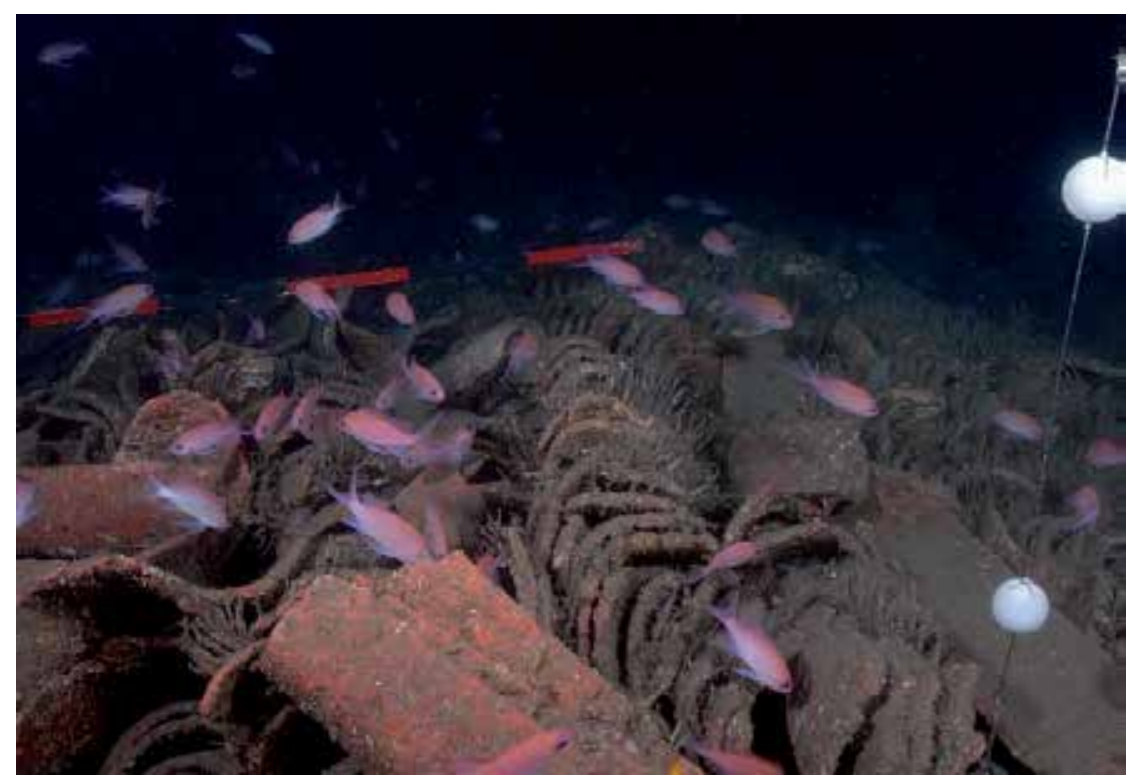

Fig. 5. Buoy for vertical reference and scale bar on a wreck in Sesimbra, Portugal. 60 meters depth, photogrammetric survey by divers during the VENUS project, 2008, Photo P. Drap. 


\section{Cartography: GIS representation}

For many years geographic information systems (GIS) have become common tools for archaeologists who see in this technology an alliance between the huge amount of information collected in the field and its graphical representation which supports the analysis. GIS graphical representations most often originate from cartography, that is to say the merging of vectors, images, and symbology using $2 \mathrm{D}$ visualization tools. The old culture of chart reading (see Christian Jacob's book on this subject, (Jacob, 1992)) is very useful in GIS but probably one of the obstacles in the way of a truly 3D GIS. As a matter of fact, even without realistic representation, the strength of GIS is linked to the symbolic cartographic representation of the data and offers a synthetic expression of the data analysis.

If $2 \mathrm{D}$ representation is sufficient to demonstrate small scale archaeological work applied to a period for which traces of the elevations do not exist, it is far from being true when one is studying a building, or in this present case, a wreck. The need for 3D representation is then of first importance and the global understanding of the study revolves around this kind of representation.

The system presented here produces 3D models of artifacts studied and their geometric patterns associated to an archaeological database (see figure 6) (Drap \& Long, 2005; Drap et al., 2008).

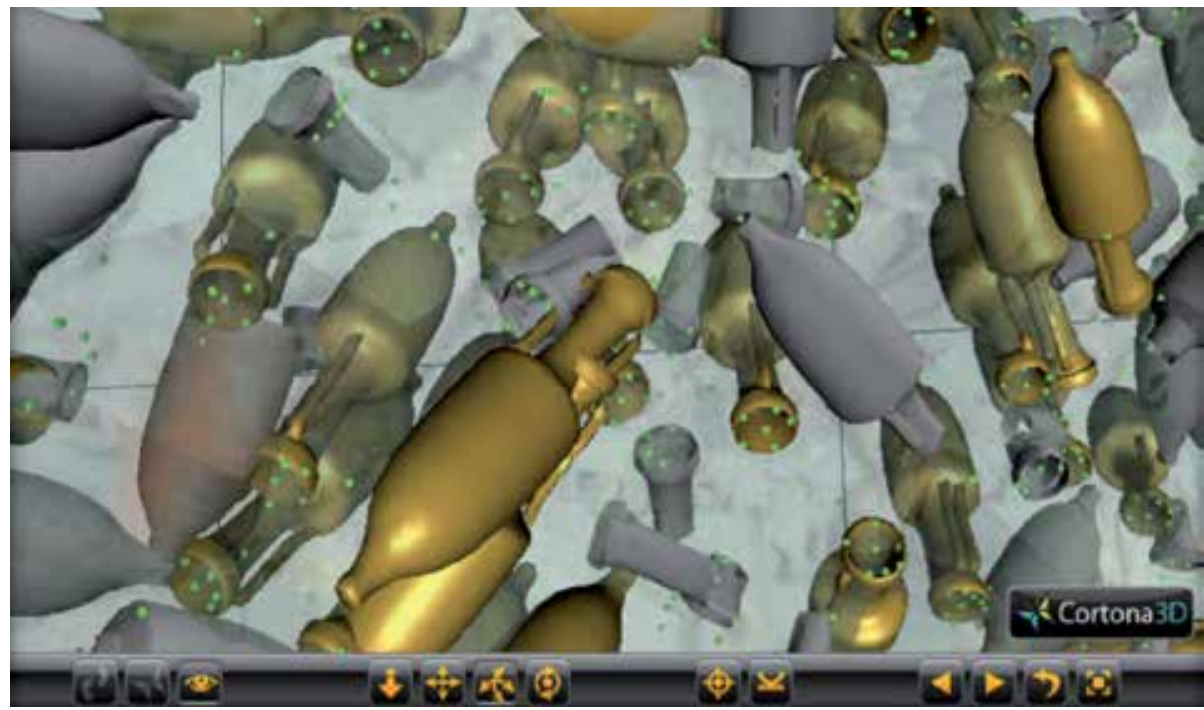

Fig. 6. 3D reconstruction of amphorae and their representation in VRML.

The link between geometry and knowledge is performed through annotations by the archaeologist and remains potentially subject to revision. The most important dimension of this work is the possible exploitation of the survey in a virtual reality system where this model interfaces with digital archaeological data. This use case may be relevant in 3D imaging, but of course also in 2D representations. In all cases, the approach is related to geographic information systems and the existing GIS standard enables the development of specific applications based on GIS concepts. 
In the context of archaeological surveys, 2D representations are well known and largely used by archaeologists. These representations can be handmade drawings or digital maps as those used in computer-aided design (CAD) systems or in geographic information systems (GIS) (Peled, 2000).

The complete digital warehouse managing all the data collected on the site (all 3D information such as seabed DTM, oriented photographs, 3D artifact reconstruction, ROV navigation, etc.) allows us to automatically build a GIS representation of the surveyed site. A 2D GIS representation has two main advantages:

- $\quad$ The 2D representation is convenient for archaeologist needs

- A GIS enables archeologists to enhance a simple geometric representation with knowledge

As often with underwater archaeology, we are always dealing with the surface layer only, especially in deep water surveys, standard tools for terrestrial 2D GIS can be very useful.

Such a GIS representation relies on standardized formats: the GeoTIFF and the Shapefile (Shapefile is a geospatial vector file format from ESRI ${ }^{\mathrm{TM}}$ but it is an open specification and is used by numerous GIS software applications including open source projects). The Shapefile format covers simple 2D geometry representation and is suitable for a schematic representation of the measured objects. The GeoTIFF format is also able to store georeferenced metadata of the image (see figure 14).

\subsection{D hand-drawn like representations of 3D artifacts}

The generation of 2D hand-drawn like representations from 3D models is part of the wide domain of non-photorealistic rendering (NPR) of 3D models. Basically, this domain aims at providing computer techniques used to display 3D or 2D (digital images) models in artistic styles (this representation is also known as emotional design), which can be very different
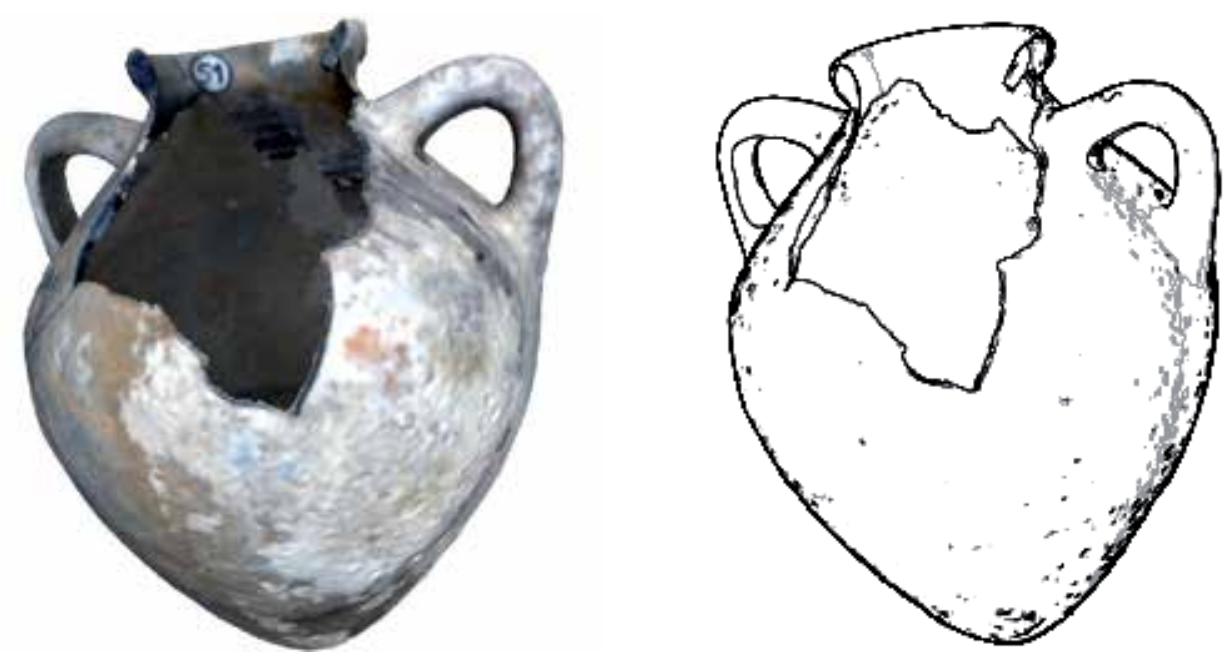

Fig. 7. An Etruscan amphora, typology Py4, from the Grand Ribaud F wreck. On the left, a 3D scan made in structured light using the Mephisto system. On the right, an NPR image made using the Judd algorithm (Judd et al., 2007). 
from reality. See (http://www.cs.utah.edu/npr/papers.html) for a long list of papers on this topic. Several methods directly devoted or potentially applicable to the representation of 3D archaeological artifacts using NPR techniques have been discussed during the last decades. See (Jardim \& De Figueiredo, 2010; Roussou \& Drettakis, 2003; Tao et al., 2009) and (Judd et al., 2007) for a very interesting open source approach of NPR applied to a 3D model build with mesh and a fine implementation called Suggestive contour software (DeCarlo \& Rusinkiewicz, 2007).

We have tested this algorithm (see figure below) on a 3D Etruscan amphora model extracted from the Grand Ribaud F.

\subsection{D representation generated from 3D models}

The underwater sites surveyed deliver an important set of 3D data including spatial references and morphological descriptions of all the artifacts observed. These are key data for building 3D models and then 2D hand-drawn like documents, closely resembling the documents commonly used by archaeologists.

We start by an inventory of graphic conventions used in underwater archeology graphic representation by analyzing published maps.

We have identified three main rules that have guided our development:

- Only the outlines of the functional parts of the artifact (bellies, handles) are represented.

- Artifact contours are drawn from a single point of view. In other words, only the visible parts are represented.

- The round parts are highlighted by a small line drawn close to the segmented contour, and according to the point of view of the artifact's orientation.

The data required as input are:

- A set of labeled triangles: each triangle (and its respective vertices) of amphorae - or fragments - is labeled. This indicates the specific amphora zone as 'internal', 'external', 'Handle1", "Handle2", etc.

- A point of view: the point of view used to generate the 2D projection of the 3D model. In our study, we chose the projection vector $(0,0,-1)$, which corresponds to an orthographic view along the horizontal plane.

From these data, and of course the measured 3D points used to position and orient the amphorae in the site reference system (see figure 6, in green), we have developed a process following the three previous rules through a combination of well-known algorithms in computer graphics. This operation required several steps.

First, an algorithm designed to manage hidden lines (hidden line removal) was used to solve the problem with the global visibility of the area concerned; that is to say, certain parts should not appear depending on the point of view. It is an extension of wireframe representations.

The hidden line algorithm we developed is an adaptation of an existing algorithm (Ammeraal \& Zhang, 2007). In Figure 9 this algorithm is applied to a 3D model of an amphora visible in Figure 8. After this step, we stored the data in a format able to manage the triangle data. 


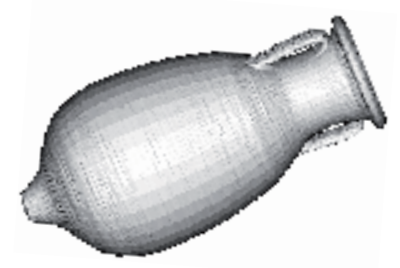

Fig. 8. Triangulated model of an amphora.

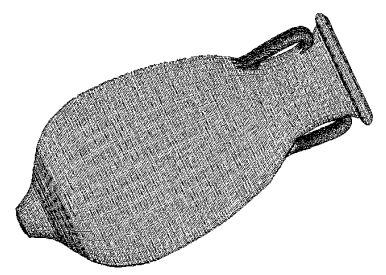

Fig. 9. Hidden line removal applied to a triangulated model.

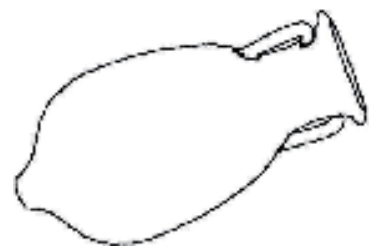

Fig. 10. Relevant lines for 2D representation.

The second step is to obtain the outline of the artifact's functional parts. This is done using the data produced by the hidden line removal algorithm. By projecting each triangle on a plane and using the Boolean union of the projected triangle according to its label, we can produce a set of shapes corresponding to the relevant part of each artifact as shown in Figure 10.

Finally we produce a set of small lines to highlight round parts of the artifact by computing the angle between the normal outline of the triangle and the projection vector, resulting in the image shown in Figure 11.

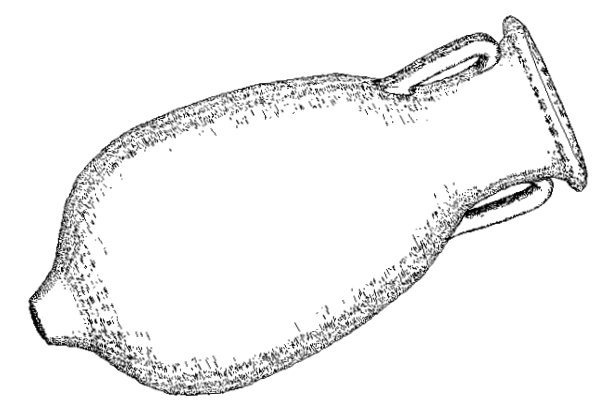

Fig. 11. Result of the hidden line algorithm rendering of the amphora shown in Figure 8. 


\subsection{Amphora fragments}

3D amphora fragments are reconstructed by means of three types of data: a set of digitized points suitably approximating the fragment geometry, the fragment type (belly, bottom, neck) and its related amphora typology, in addition to the database of 3D amphorae constructed in the first step of the process.

From these data, a fragment reconstruction technique has been developed (see figure 12). For each fragment, the goal is to find the part of the related amphora model "suitably" corresponding to the geometrical shape of the fragment. To do this, we investigated the potential of using a matching-based technique. This is defined as the two-step process. The first step consists in fitting the fragment contour to the amphora model. The second aims at extracting the part of the amphora lying "inside" the matched contour.

3D points representing the fragment geometry are obtained using a photogrammetric interactive process, and describe the geometry of each fragment and their orientation with respect to the seabed (see figure below).
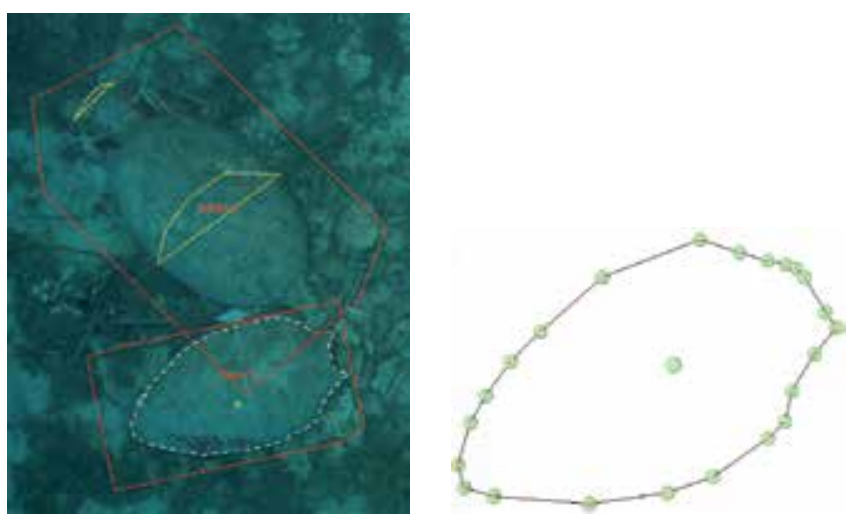

Fig. 12. On the left, the original image used to measure, by photogrammetry, the points belonging to the studied fragment. On the right, 23 points measured on the fragment's perimeter.

To obtain a satisfactory compromise between the accuracy and the size of the description while considering the potential flaws inherent to the photogrammetric interactive process, the measurement protocol we opted for was to collect for each fragment $n$ points along its contour and one point located around its "center". This task was manually achieved by expert archaeologists using ARPENTEUR software (Drap et al., 2004). The result (for each fragment) corresponds to a 3D closed polyline, a 3D point lying "inside" the fragment, and semantic data such as the potential amphora part (e.g. a belly fragment or a handle fragment), and a potential amphora type to which the fragment may belong (see figure 12).

\subsection{Matching 3D measured points on 3D amphora models}

The problem of finding a satisfactory rigid registration of the measured points of a given fragment to a set of points belonging to its related amphora model has been studied as a key element for the $3 \mathrm{D}$ fragment generation. A formalization of the problem has led this 
investigation to the theoretical problem of finding a similitude of scale 1.0, (i.e. a translation vector $t$, and a $3 * 3$ rotation matrix $R$ ) in order to transform the $3 \mathrm{D}$ fragment perimeter into a polygonal path belonging to the amphora model.

Due to the discrete nature of the data to match, ICP-like algorithms (ICP for Iterative Closest Point), which are widely used to handle this kind of registration problem, were investigated as a potential solution. The process has been optimized by a) suitably reducing the number of points of the perimeter (reduced to 4 points for the fragment contour and an additional point for the convexity information), b) geometrically improving the way of coupling points (taking into account the angle and the length of the fragment perimeter edges for cutting the initial combinatory logic involved by the high number of data of the 3D amphora models), and c) suitably configuring the parameters controlling the minimization process (number of iterations, convergence ratio, maximum errors between the contouring points and the amphora model points).

The optimized method results in accurate matches in only a few seconds, and at the most, a few minutes. Once the five points are matched, the corresponding transformation is applied to the initial perimeter as shown in the figure below.
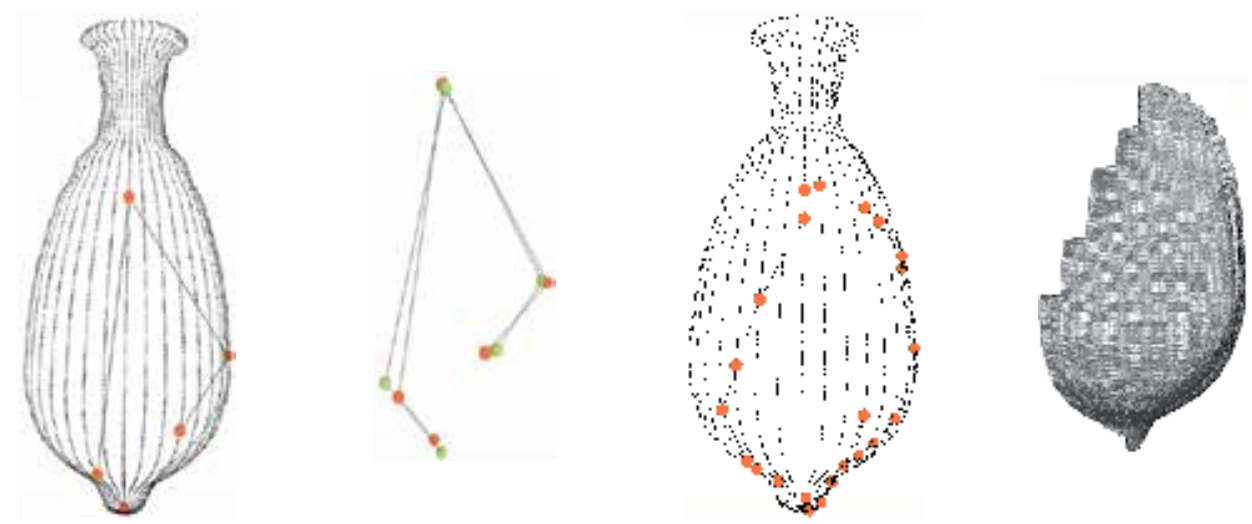

Fig. 13. Extracting triangles lying inside the matched perimeter and closing the fragment.
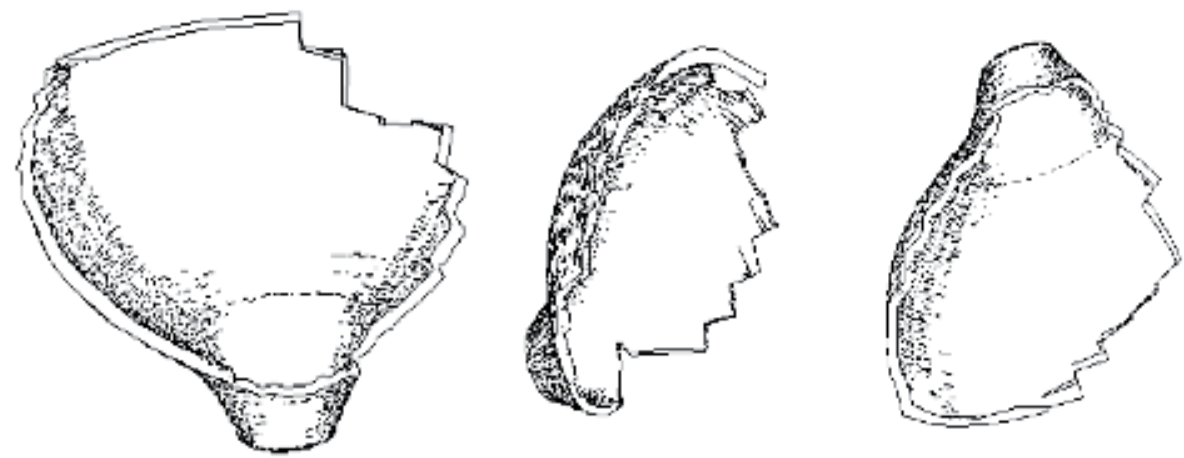

Fig. 14. 2D representation automatically produced using 3D simulation of a traditional archaeological drawing. Different points of view of the same artifact in 3D. 


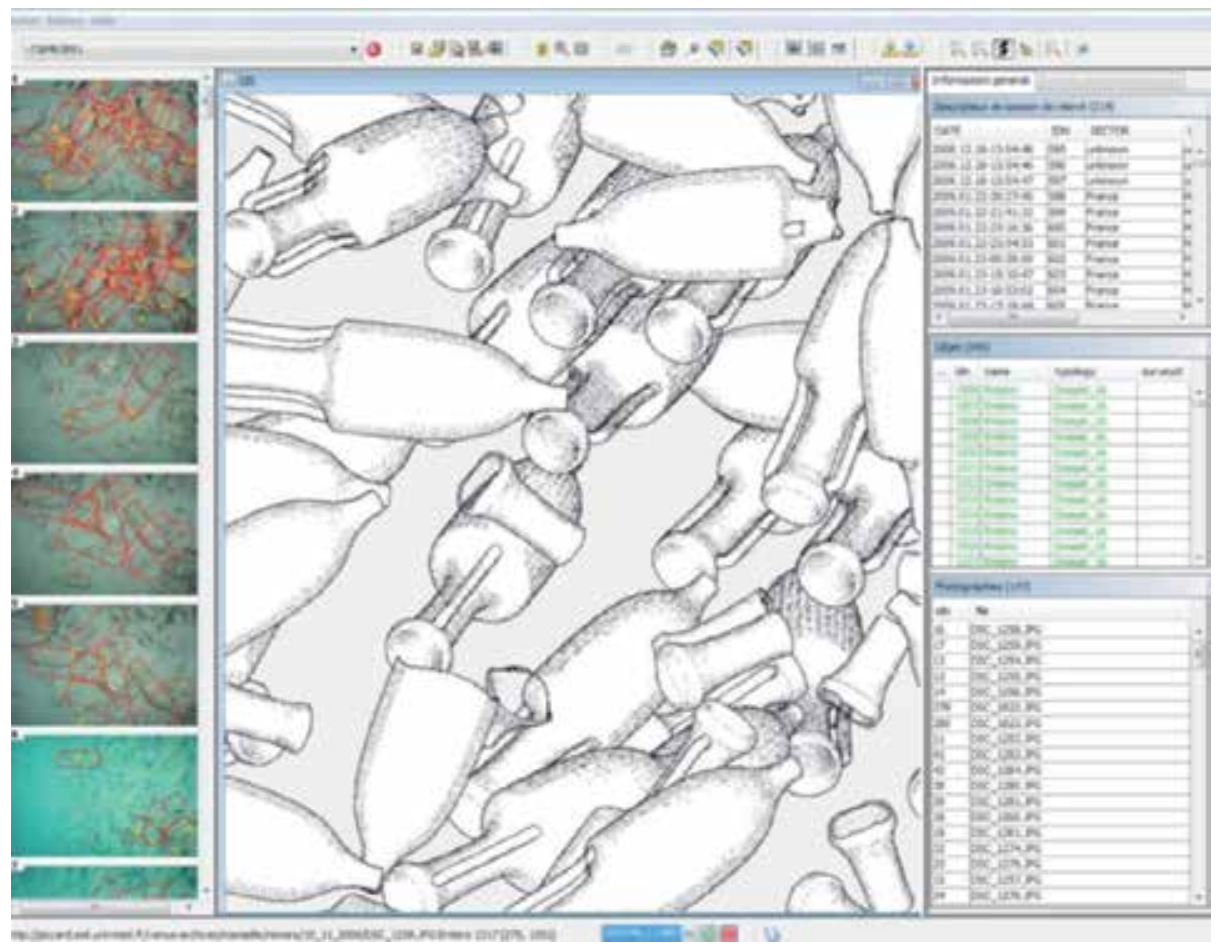

Fig. 15. 2D representation generated from the computed 3D models. The use of a standard Shapefile from ESRI ${ }^{\mathrm{TM}}$ enables interaction with archaeological data as well as photogrammetric data using standard GIS software.

\section{A survey as a cloud of 3D points?}

Mapping, archaeological surveys, and more generally, surveys dedicated to cultural heritage are an interpretation of the real world and not a simple exercise in data acquisition that is more or less metric.

Taking into account the interpretation of the real world during the process of archaeological surveys is similar to the work of the artist: Knowledge always guides the survey as the emotion guides the action.

New advances in photogrammetry and the capability to produce dense 3D point clouds do not solve the problem of surveys. New opportunities for 3D representation are now available and we must to use them and find new ways to link geometry and knowledge.

\subsection{Automatic survey by photogrammetry}

The photogrammetric process is a very efficient procedure consisting mainly in two phases. The first phase is data acquisition by photographs which requires light processing and is nonintrusive (remote sensing), not time consuming (only the time necessary to take pictures), and potentially a quite thorough practice. The second phase is further data elaboration, carried out in a laboratory. The latter phase is always manual and time consuming. 
In case of photographs taken by an ROV, it is possible to minimize the time and costs of photo elaboration, taking advantage of the way underwater photographs are taken (often by strip with an overlap from one photo to the next).

Using the SIFT (Lowe, 2004; Munich et al., 2006) algorithm to detect homologous points, we will make all the possible relative orientations using the Stewenius algorithm (Kalantari et al., 2011; Nister, 2004; Stewenius et al., 2006).

Once we have automatically detected a set of homologous points on the photographs, we compute all the possible relative orientations by looking for common points between the possible pairs.

A pre-orientation computation based on the relative orientation of the common points of stereoscopic image pairs is developed, which will enable the computation of all photograph orientation data in a unique reference system.

This phase will be the key to start the bundle adjustment and compute the final external orientation necessary for the surface densification and orthophoto generation.

This task, from homologous point determination, bundle adjustment and the generation of dense point clouds is now fully automatic thanks to the work on bundle adjustment of Manolis Lourakis (Lourakis \& Argyros, 2009) and Noah Snavely (Snavely et al.) for the Bundler software and finally Ponce and Furukawa for the point cloud generation (Agarwal et al., 2010; Furukawa \& Ponce, 2008, 2010).

This software suite is now open source and freely available on the Internet.

A very interesting application is being developed by the IGN in France and offers dense 3D motion capture software which is also open source (Pierrot-Deseilligny \& Cléry, 2011). Their approach is more rigorous, from a photogrammetric point of view, and allows using calibrated cameras.

This dense map approach makes it possible to obtain a dense and accurate 3D points cloud with colour information directly extracted from the photographs.

\subsection{Large scale detail by photogrammetry}

We also tested as part of this mission approach automatic mapping proposed by Furukawa and Ponce (Furukawa \& Ponce, 2010). We tested this approach on large-scale details, Gorgonaria whose ends were slightly in motion because of the water current, see figure below.

The Gorgonaria colonies presented here is about $30 \mathrm{~cm}$ length and we are close to a macro scale approach.

One camera was used and sixty photographs were taken for each of the tests that follow. The study of the accuracy and the percentage of coverage were not yet completed at the time of publication due to the fact that the study dates only from April 2011.

\subsection{The "Liban" wreck}

The Liban is a ship built in 1882 in Glasgow (Scotland), measuring 91 meters long and 11 wide. It was equipped with a steam engine. On June 7,1903 at noon, the Liban left the port of Marseille and less than one hour later sunk after a big collision with another ship. 

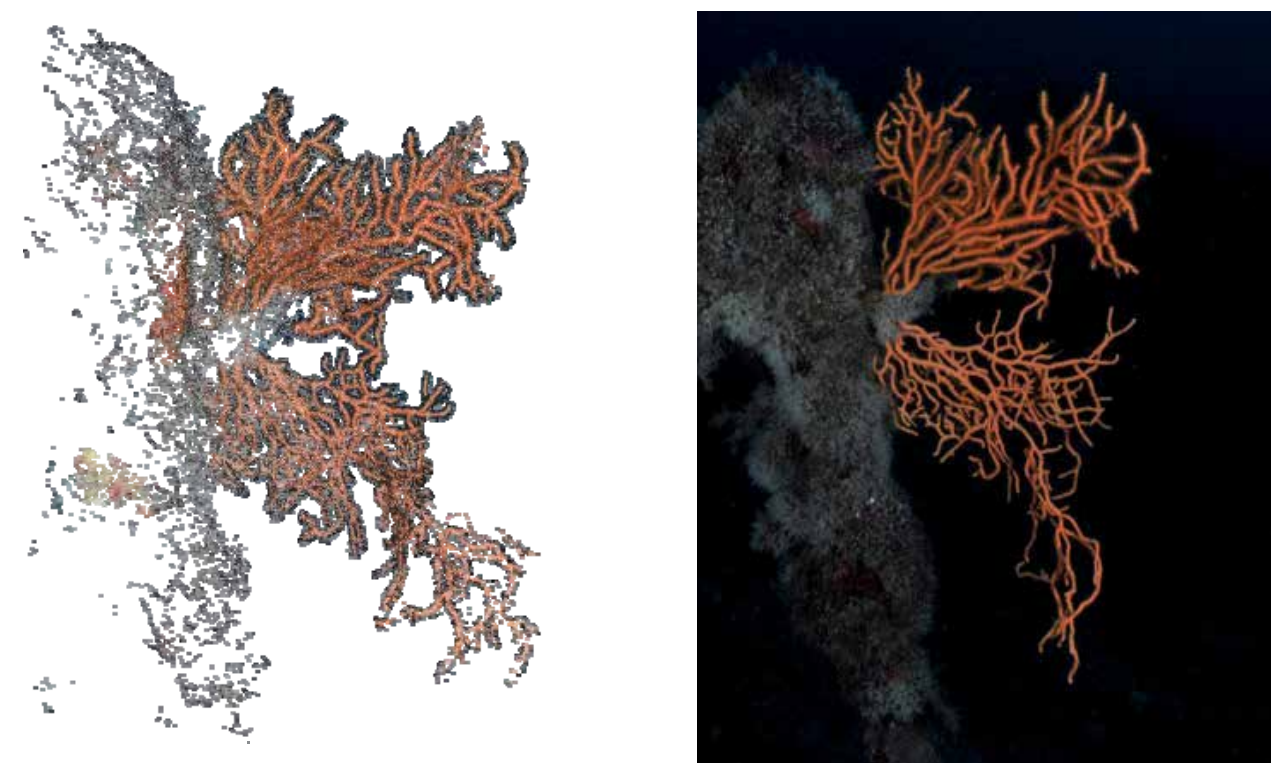

Fig. 16. On the right, one of the 64 digital photographs taken. On the left, the point cloud automatically computed using the Furukawa method.

Today, the Liban is a very attractive dive site close to Marseilles at 30 meters deep. We chose to survey the bow in order to develop and test our approach. Three dives and almost 2000 photos were necessary to obtain the 3D point cloud visible in Figures 17 and 18.
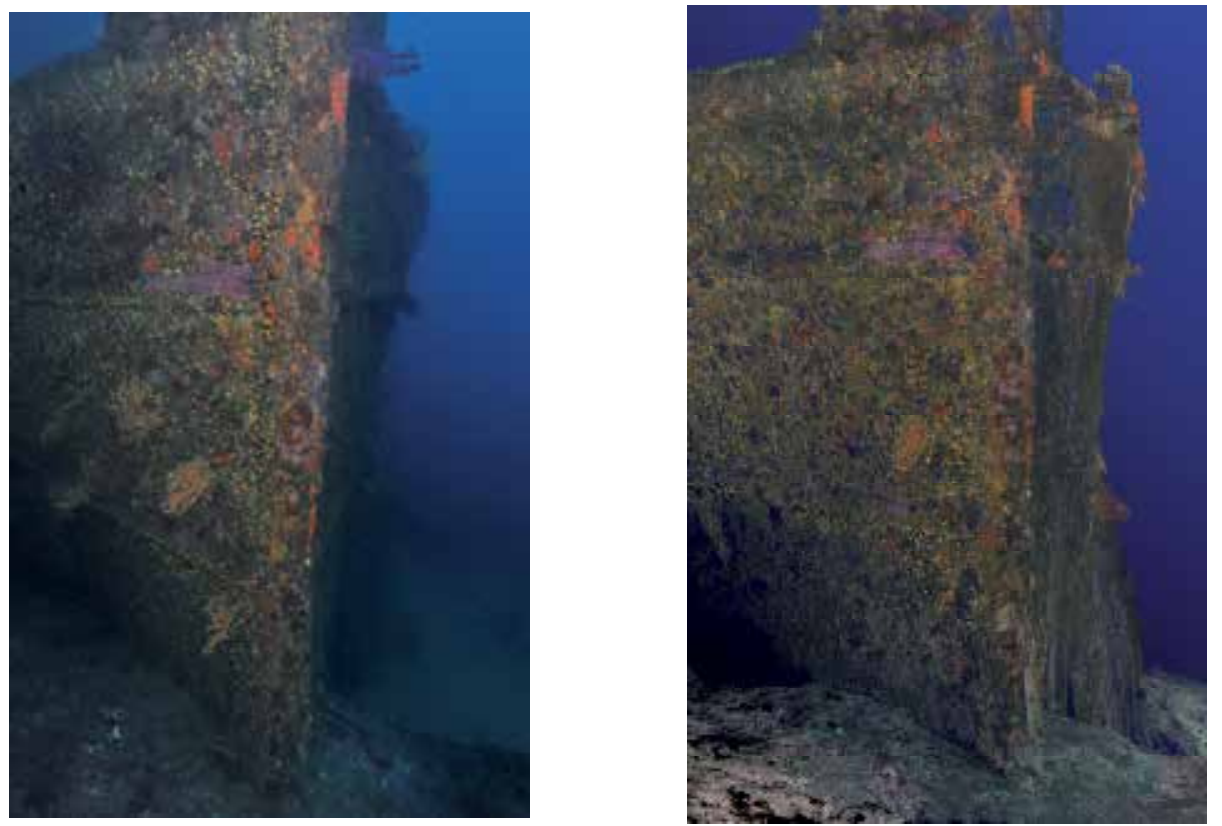

Fig. 17. On the left, one of the 1,828 digital photographs taken. On the right, the Liban wreck: cloud of 3D points measured by photogrammetry using the Furukawa method. 

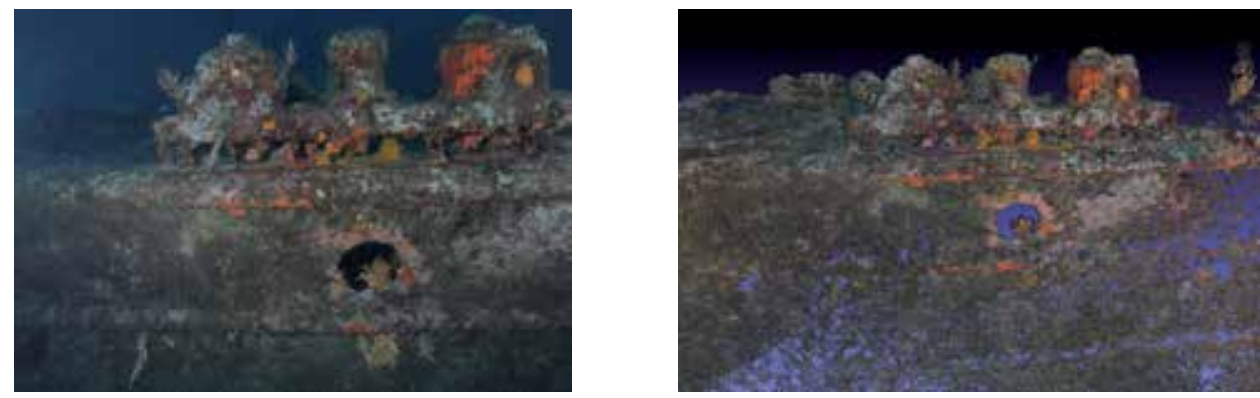

Fig. 18. On the left, one of the 1,828 digital photographs taken. On the right, the Liban wreck: cloud of 3D points measured by photogrammetry using the Furukawa method.

\section{Conclusion}

Using the computerized recordings of all excavation data, archaeologists are now able to obtain accurate $2 \mathrm{D}$ or $3 \mathrm{D}$ representations and interact using faithful facsimiles built close to the ground and viewable using standard software.

But beyond the process of underwater photogrammetry dedicated to artifacts, we must remember the concept of a template, or theoretical model, from which facsimiles of artifacts are generated from a limited number of measured points that provide their positions, orientations and geometrical parameters. Namely, all the visible amphorae and fragments are modeled from a concept, enriched by the measurements of each object, with its own attributes and characteristics. It is this principle, founded on rationality, which gives an archaeologist the possibility to view an entire site, which so far he has only seen a few fragmented views through a diving mask.

A rule-based engine manages the computation of intrinsic attributes according to a given geometrical primitive measured by an expert. Thus the resulting model supports to varying degrees the merging of measurements and theoretical knowledge. The final model can offer a complete graphic representation of an object seen only partially (in archeological sites, objects can almost never be seen completely because they are usually partially destroyed or eroded).

Of course, even if the representation of artifacts is complete, it is always possible to know whether an attribute has been measured or if its value comes from the theoretical model.

Persistent data is based on the notion of a theoretical model of the objects identified. We use a hybrid relational and semi-structured language (MySQL and XML) to evolve into the near future, to a PostgreSQL system to manage spatial locations, and an XML / CIDOC Conceptual Reference Model (CRM) for greater interoperability with the world heritage.

The graphic documents, 2D or 3D, dedicated to the survey representation are also very different in nature. Their function is double; first to help understand the site by providing a comprehensive and thematic overview, second to interface with data entered by the expert, allowing reasonable access to a set of heterogeneous data.

Moreover, the survey, still done manually with annotations from experts, incorporates certain mistakes due to human factors during the measurement process or during the identification of the object or fragment. A mechanism for detecting inconsistencies and a set 
of proposals needed to restore consistency are made available to experts during the measurement phase and also in the analysis phase of the data.

Finally, the recent advances in photogrammetry and computer vision, mainly in automatic matching and dense map generation, will change the way underwater sites are surveyed. The link between measurement and knowledge will be done using both a dense cloud of 3D points and a set of oriented photographs. Under these conditions, pattern recognition will be performed both in 2D and $3 \mathrm{D}$ on images and will become more robust.

\section{Acknowledgements}

I want to thank K. Edmundson for his active participation and significant contributions regarding the bundle adjustment. I would also like to thank the PhD students who have worked with me.

I want to thank also Julien Seinturier for his development of the GIS interface, JeanChristophe Chambelland for his contributions in developing the amphorae fragments computation and Odile Papini for her strong contribution on the consistency check approach.

I also want to thank the entire COMEX team for their close and friendly collaboration for more than 10 years without forgetting Luc Long, the underwater archaeologist with whom I started working in this field.

I owe many thanks to Olivier Bianchimani for his help in the diving mission, mainly on the Liban wreck.

\section{References}

Agarwal S., Furukawa Y., Snavely N., Curless B., Seitz S., \& Szeliski R.. (2010). Reconstructing Rome. in Computer,Vol.:43, pp.40-47.

Ammeraal L., Zhangn K. (2007). Computer Graphics for Java Programmers (Willey ed.), isbn/issn: 0470031603.

American Society of Photogrammetry (1980). Manual of photogrammetry, Fourth Edition: Asprs Pubns), isbn/issn: 0937294012.

Bass G.F. (1970). Archaeology under Water (Penguin Book ed.). Harmondsworth: Pelican Book, isbn/issn: 0140212191.

Bass G.F., \& Rosencrantz D. (1973). L'utilisation des submersibles pour les recherches et la cartographie photogrammétrique sous-marine. Paper presented at L'archéologie subaquatique, une discipline naissante. Paris. pp.285-298.

Bay H., Ess A., Tuytelaars T., Van Gool L.. (2008). Speeded-Up Robust Features (SURF). in Comput. Vis. Image Underst.,Vol.:110, pp.346-359.

Blaha G. (1982). Free Networks: Minimum Norm Solution As Obtained by the Inner Adjustment Constraint Method. in Bulletin Geodesique,Vol.:56, pp.209-219.

Buchsenschutz O. (2007). Images et relevés archéologiques, de la preuve à la démonstration Paper presented at the 132e congrès national des sociétés historiques et scientifiques, Arles. Les éditions du Cths (édition électronique) (Pub.). 
Butler J.B., London E-G., Lane S.L. (2002). Through-water close range digital photogrammetry in flume and field environments. in Photogrammetric Record,Vol.:17, pp.419.

Chapman P., Bale K., Drap P. (2010). We All Live in a Virtual Submarine. in IEEE Computer Graphics and Applications, Vol.:30, pp.85-89.

Ciani J. B., Hunter E. K., Dowling, C. B., Brunk D. H. (1971). Seafloor surveying by divers. in ASCE J Surv Mapp Div, Vol.:97, pp.149-164, isbn/issn: (Ed.) Epub.

Decarlo D., Rusinkiewicz S. (2007). Highlight Lines for Conveying Shape. Paper presented at the International Symposium on Non-Photorealistic Animation and Rendering (NPAR).

Doucette J., Harvey E., Shortis M. (2002). Stereo-video observation of nearshore bedforms on a low energy beach. in Marine Geology, Vol.:189, pp.289-305.

Drap P., Seinturier J., Long L. (2003). Archaeological 3D Modelling using digital photogrammetry and Expert System. The case study of Etruscan amphorae. Paper presented at the 3IA 2003 - The Sixth International Conference on Computer Graphics and Artificial Intelligence Limoges (France).

Drap P., Durand A., Provin R., Long L.. (2005, 26 September - 1 October). Integration of Multi-Source Spatial Information And XML Information System In Underwater Archaeology. Paper presented at the CIPA 2005 XX International Symposium Torino, Italy. The Cipa International Archive. Vol.:XX-2005-2, pp.765-771.

Drap P., Grussenmeyer P., Curtinot P-Y., Seinturier J., Gaillard G. (2004, 12-23 July). Presentation of the web based ARPENTEUR tools: towards a Photogrammetry based Heritage Information System. Paper presented at the ISPRS XXth CONGRESS, Istanbul, Turkey.

Drap P., Long L. (2005). Photogrammétrie et archéologie sous-marine profonde. Le cas de l'épave étrusque Grand Ribaud F in XYZ, Vol.:Nº3, partie 1 et $\mathrm{N}^{\circ} 104$, partie 2.

Drap P., Seinturier J., Conte G., Caiti A., Scaradozzi D., Zanoli S., Gambogi P.. (2008). Underwater cartography for archaeology in the VENUS project. in Geomatica, Canadian Institute of Geomatics. The Journal of Geospatial Information Science, Technology And Practice,Vol.:62 N 4 Special issue on Marine Geomatics, pp.419-428.

Edmundson K. (1997). Sequential Estimation for Close Range Vision Metrology. The University of Melbourne, Melbourne, Australia.

Edmundson K., Fraser C.S. (1998). A practical evaluation of sequential estimation for vision metrology. in Isprs Journal of Photogrammetry and Remote Sensing, Vol.:53, pp.272285.

Faig W. (1979). Photogrammetric surveys of underwater objects. in Proceedings coastal mapping symposium, August 1978, Rockville, Maryland, in (American Society of Photogrammetry, Falls Church, VA) pp.183-187.

Fraser C.S. (1982). Optimization of Precision in Close-Range Photogrammetry. in Photogrammetric engineering and remote sensing, Vol.:48, pp.561-570.

Fryer B. J., Fraas K. C. (1986). On the calibration of Underwater Camera. in The Photogrammetric Record, Vol.:12, pp.73 85.

Furukawa Y., Ponce J. (2008, 23-28 June 2008). Accurate camera calibration from multi-view stereo and bundle adjustment. Paper presented at the Computer Vision and Pattern Recognition, 2008. CVPR 2008. IEEE Conference on. pp.1-8, isbn/issn:1063-6919.

Furukawa Y., Ponce J. (2010). Accurate, Dense, and Robust Multiview Stereopsis. in IEEE Transactions on Pattern Analysis and Machine Intelligence, ,Vol.:32, pp.1362-1376. 
Fusiello A., Murino V. (2004). Augmented Scene Modeling and Visualization by Optical and Acoustic Sensor Integration. in IEEE transaction on Visualization and computer graphics, Vol.:10, pp.625-636.

Harris E.C. (1979). Principles of Archaeological Stratigraphy (Academic Press ed.). London.

Hesnard A.. (1988). L'épave romaine Grand Ribaud D/ Archaeonautica ed.

Hoehle J. (1971). Reconstruction of the underwater object. in Photogrammetry Eng, Vol.:37, pp.948-954.

Hurtós N., Cufí X., Salvi J. (2010). Integration of optical and acoustic sensors for D underwater scene reconstruction. in Instrumentation Viewpoint.

Jacob C. (1992). L'empire des cartes. Paris, isbn/issn: 2-226-06083-9.

Jardim E., De Figueiredo L.H. (2010). A Hybrid Method for Computing Apparent Ridges, 23rd Conference on Graphics, Patterns and Images (SIBGRAPI), Editor(Ed.), pp. 118125. Gramado CPS Conference Publishing Service(Pub.).

Judd T., Durand F., Adelson E. (2007). Apparent Ridges for Line Drawings. in ACM Transactions on Graphics, Proceedings of SIGGRAPH 2007,Vol.:26, pp.19.

Kalantari M., Hashemi A., Jung F., Guédon J.-P. (2011). A New Solution to the Relative Orientation Problem Using Only 3 Points and the Vertical Direction. in Journal of Mathematical Imaging and Vision archive, Vol.:39.

Kalia R., Lee K., B.V.R. S., Je S.K., Oh W.G.. (2011, 9-11 Fev.). An analysis of the effect of different image preprocessing techniques on the performance of SURF: Speeded Up Robust Feature. Paper presented at the 17th Korea-Japan Joint Workshop on Frontiers of Computer Vision (FCV). pp.1-6.

Kwon Y. (1998). Refraction Error. Web page available: http://www.kwon3d.com/theory/dlt/refr.html Last Access date: 20111002.

Lavest J. M., Rives G., Lapresté J. T. (2003). Dry camera calibration for underwater applications. in Machine Vision and Applications, Vol.:13, pp.245-253.

Liou B. (1973). Recherches archéologiques sous-marines. in Gallia,Vol.:31, pp.571-608.

Long L. (1995). Protection du Patrimoine Archéologique Sous-Marin en Méditerranée.100 sites d'intérêt commun méditerranéen. Paper presented at the Documents techniques, Marseille. pp.14-46.

Lourakis M., Argyros A.. (2009). SBA: A software package for generic sparse bundle adjustment. in ACM Trans. Math. Softw., ,Vol.:36, pp.1-30.

Lowe D. (2004). Distinctive image features from scale-invariant keypoints. in International Journal of Computer Vision, Vol.:60, pp.91-110.

Maas H.-G. (2000). New developments in Multimedia Photogrammetry. Paper presented at the Institute of Geodesy and Photogrammetry, Swiss Federal Institute of Technology, Zurich.

Munich M. E., Pirjanian P., Di Bernardo E., Goncalves L., Karlsson N., Lowe D. (2006). SIFTing through features with ViPR - Application of visual pattern recognition to robotics and automation. in IEEE Robotics \& Automation Magazine, Vol.:13, pp.72-77.

Nicosevici T., Gracias N., Negahdaripour S., Garcia R. (2009). Efficient three-dimensional scene modeling and mosaicing. in Journal of Field Robotics, Vol.:26, pp.759-788.

Nister D. (2004). An efficient Solution to the Five-point Relative Pose Problem. in IEEE Transactions on Pattern Analysis and Machine Intelligence, Vol.:26, pp.756 770. 
Papini O., Drap P. (2009, july). The Revision of Partially Preordered Information in Answer Set Programming. Paper presented at the Proceedings of ECSQARU, Lecture Notes in Computer Science, Verona. pp.421-433.

Peled A. (2000). Three-dimensional digital photogrammetric update of the israeli national gis data base. Paper presented at the XIXth Congress of the International Society for Photogrammetry and Remote Sensing (ISPRS) geoinformation for all, Amsterdam. Vol.:XXXIII, pp.443, 448., Archives of Photogrammetry and Remote Sensing (Pub.).

Pierrot-Deseilligny M., Cléry I. (2011, 2-4 March). APERO, an Open Source Bundle Adjusment Software for Automatic Calibration and Orientation of a Set of Images. Paper presented at the 3D Arch 2011, Trento, Italy. ISPRS Commission V Symposium, Image Engineering and Vision Metrology (Pub.).

Pollio J. (1968). Application of underwater photogrammetry. in Naval Oceanographic Office pp.46.

Pollio J. (1971). Underwater Mapping with Photography and SONAR. in Photogramm Eng, Vol.:37, pp.955-968.

Roman C., Inglis G., Rutter J. (2010). Application of structured light imaging for high resolution mapping of underwater archaeological sites. in IEEE OCEANS

Roussou M., Drettakis G.. (2003). Photorealism and Non-Photorealism in Virtual Heritage Representation. In Chalmers Alan \& Arnold David \& Franco Niccolucci, First Eurographics Workshop on Graphics and Cultural Heritage, Editor(Ed.), pp. 51-60. Brighton, United Kingdom: Eurographics Association(Pub.).

Seinturier J. (2007). Fusion de connaissances : Applications aux relevés photogrammétriques de fouilles archéologiques sous-marines. Université du Sud Toulon Var, Toulon.

Sérayet M., Drap P., Papini O.. (2011). Extending Removed Sets Revision to partially preordered belief bases. in International journal of approximate reasoning, Vol.:52, pp.110-126.

Shortis M., Harvey E., Seager J.. (2007, 28 January-1 February 2007). A Review of the Status and Trends in Underwater Videometric Measurement. Paper presented at the SPIE Conference 6491, Videometrics IX, ISET/SPIE Electronic Imaging, San Jose, California, U.S.A.

Shortis M., Harvey E., Abdo D. (2009). A Review Of Underwater Stereo-Image Measurement For Marine Biology And Ecology Applications. In Oceanography and Marine Biology: An Annual Review, Vol 47. Vol.: 47, pp. 257-292, Crc Press-Taylor \& Francis Group(Pub.), isbn/issn:0078-3218.

Singh H., Roman C., Whitcomb L., Yoerger D. (2000). Advances in Fusion of High Resolution Underwater Optical and Acoustic Data. Paper presented at the Proceedings of the 2000 International Symposium on Underwater Technology, Tokyo Japan.

Snavely N., Simon I., Goesele M., Szeliski R., Seitz S. M.. (2010). Scene Reconstruction and Visualization From Community Photo Collections. in Proceedings of the Ieee,Vol.:98, pp.1370-1390.

Stewenius H., Engels C., Nister D.. (2006). Recent developments on direct relative orientation. in Isprs Journal of Photogrammetry and Remote Sensing, Vol::60, pp.284294.

Tao L., Renju L., Hongbin Z. (2009). 3D line drawing for archaeological illustration, Computer Vision Workshops (ICCV Workshops), Editor(Ed.), pp. 907-914. 
Tchernia A., Pomey P., Hesnard A. (1978). L'épave romaine de La Madrague de Giens (Var). in Supplément à Gallia $\mathrm{N}^{\circ} 34$.

Telem G., Filin S. (2010). Photogrammetric modeling of underwater environments. in ISPRS Journal of Photogrammetry and Remote Sensing, Vol.:65, pp.433-444. 



\section{Edited by Daniel Carneiro da Silva}

Photogrammetry is widely accepted as one of the best surveying methods to acquire tridimensional data without direct contact with the object, but its high operational costs in equipment and personnel somewhat limit its application in mapping. However, with the development of digital photogrammetry in the 1990's, it was possible to introduce automated processes and reduce the personnel costs. In the following years, the cost of computer hardware, digital cameras and positioning sensors has been lowering, making photogrammetry more accessible to other engineering fields, such as architecture, archeology and health fields. This book shows the results of the work of researchers from different professional backgrounds, which evaluate the uses of photogrammetry, including issues of the data, processing, as well as the solutions developed for some surveying types that can be extended to many applications. 